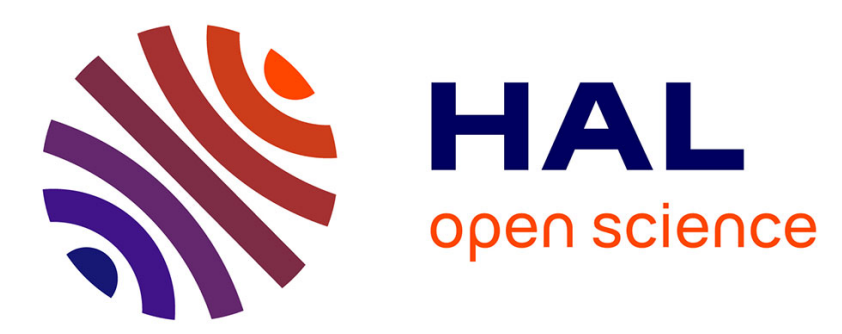

\title{
Gamma spectroscopy with AGATA in its first phases: New insights in nuclear excitations along the nuclear chart
}

\author{
A. Bracco, G. Duchêne, Zs. Podolyák, P. Reiter
}

\section{- To cite this version:}

A. Bracco, G. Duchêne, Zs. Podolyák, P. Reiter. Gamma spectroscopy with AGATA in its first phases: New insights in nuclear excitations along the nuclear chart. Prog.Part.Nucl.Phys., 2021, 121, pp.103887. 10.1016/j.ppnp.2021.103887 . hal-03371238

\section{HAL Id: hal-03371238 \\ https://hal.science/hal-03371238}

Submitted on 22 Oct 2021

HAL is a multi-disciplinary open access archive for the deposit and dissemination of scientific research documents, whether they are published or not. The documents may come from teaching and research institutions in France or abroad, or from public or private research centers.
L'archive ouverte pluridisciplinaire HAL, est destinée au dépôt et à la diffusion de documents scientifiques de niveau recherche, publiés ou non, émanant des établissements d'enseignement et de recherche français ou étrangers, des laboratoires publics ou privés. 
Review

\title{
Gamma spectroscopy with AGATA in its first phases: New insights in nuclear excitations along the nuclear chart
}

\author{
A. Bracco ${ }^{\mathrm{a}, \mathrm{b}, *}$, G. Duchêne ${ }^{\mathrm{c}}$, Zs. Podolyák $^{\mathrm{d}}$, P. Reiter ${ }^{\mathrm{e}}$ \\ ${ }^{a}$ Dipartimento di Fisica dell'Università degli Studi di Milano, Italy \\ ${ }^{\mathrm{b}}$ INFN, Sezione di Milano, Italy \\ ' Université de Strasbourg, CNRS, IPHC UMR 7178, F-67000 Strasbourg, France \\ d Department of Physics, University of Surrey, Guildford, GU2 7XH, United Kingdom \\ ${ }^{\mathrm{e}}$ Institute of Nuclear Physics, University of Cologne, 50937 Cologne, Germany
}

\section{A R T I C L E I N F O}

\section{Article history:}

Available online 11 June 2021

\section{Keywords:}

Nuclear structure

Gamma spectroscopy

Nuclei far from stability

\begin{abstract}
A B S T R A C T
The Advanced GAmma Tracking Array (AGATA), the new generation high-resolution $\gamma$ ray spectrometer, has seen the realization of the first phases of its construction and exploitation. A number of nuclear structure studies based on experiments utilizing the principle of $\gamma$-ray tracking were carried out in this decade. The combination of highest detection efficiency and position sensitivity allowed very selective spectroscopic studies with stable beams and the use of instable ion beams with the lowest intensities. Nuclear-structure studies commenced already at INFN-LNL (Legnaro, Italy) with a first implementation of the array consisting of five AGATA modules. A larger array of AGATA modules was used at GSI (Darmstadt, Germany) for experiments with unstable ion beams at relativistic energies. The spectrometer was then mounted in a beam line at GANIL (Caen, France). This review discusses several of the obtained results, underlying the progress made and future perspectives. The performed experiments give insights into nuclear structure issues which are connected to single particles, collective degrees of freedom, nucleon interactions and symmetries. Most of the investigated nuclei are located outside the stability line and for stable nuclei the investigations concern unexplored configurations. Altogether the obtained results represent advances which could test theory in exclusive way and motivate new theoretical developments. Opportunities for further $\gamma$-ray spectroscopy with the foreseen more advanced phase of the AGATA emerge in the discussions of the presented data.
\end{abstract}

(c) 2021 Published by Elsevier B.V.

\section{Contents}

1. Introduction

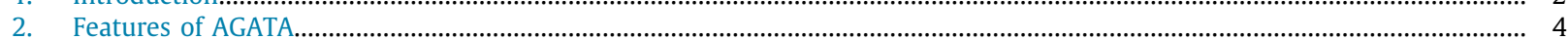

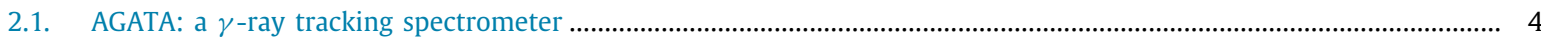

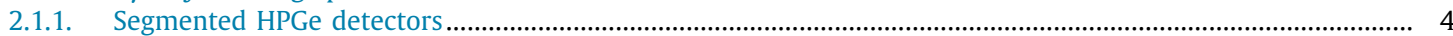

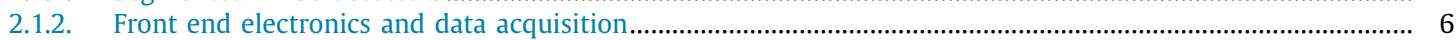

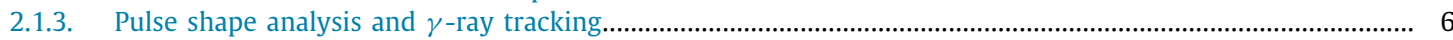

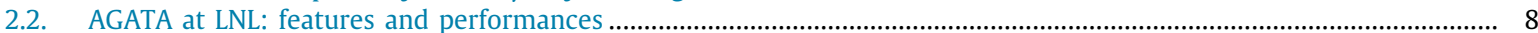

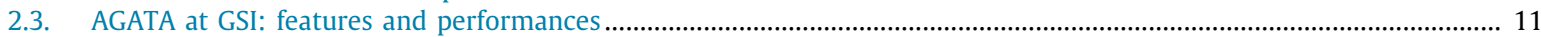

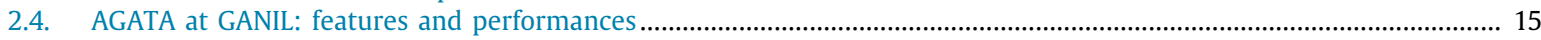

\footnotetext{
* Corresponding author at: Dipartimento di Fisica dell'Università degli Studi di Milano, Italy.

E-mail address: Angela.Bracco@mi.infn.it (A. Bracco).
} 


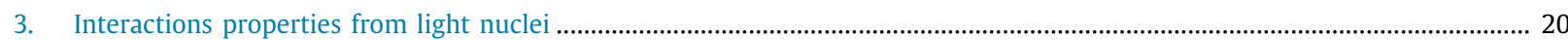

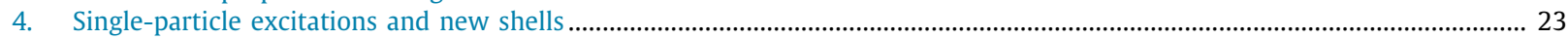

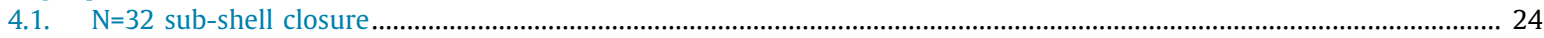

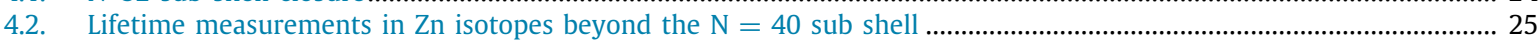

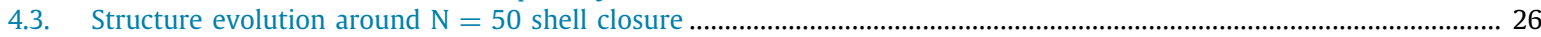

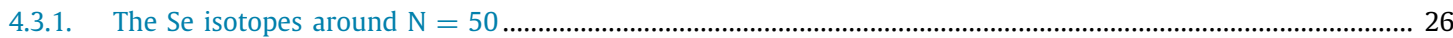

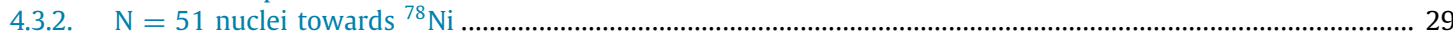

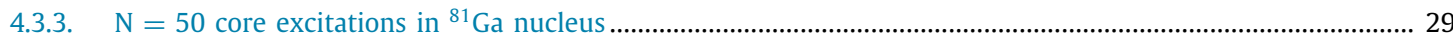

4.4. Structure evolution in the neutron-deficient semi-magic tin isotopes...................................................................... 31

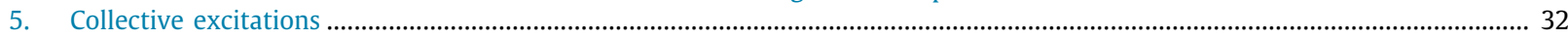

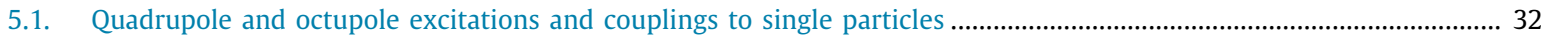

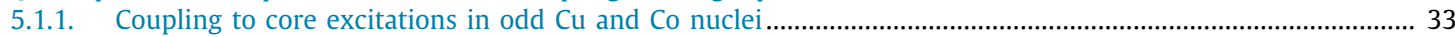

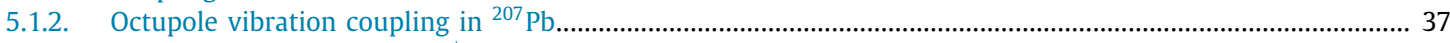

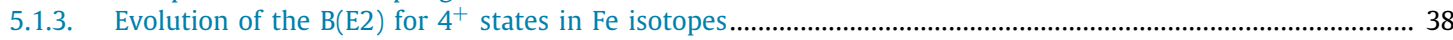

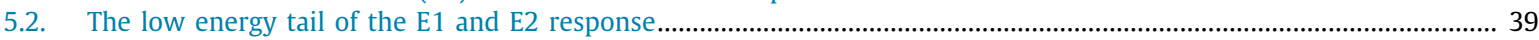

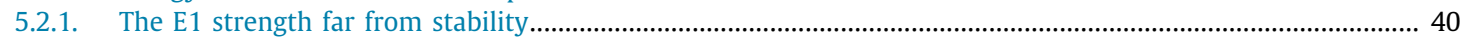

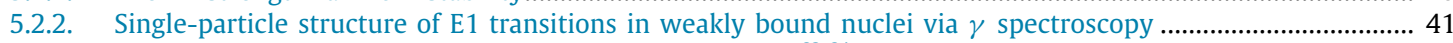

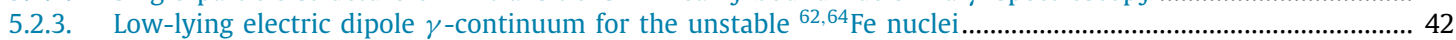

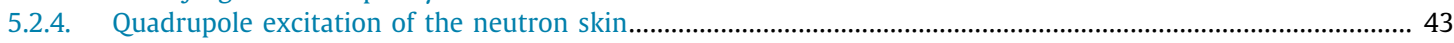

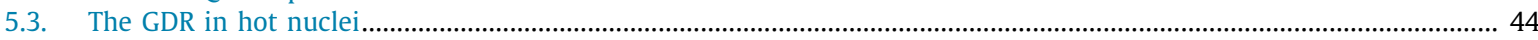

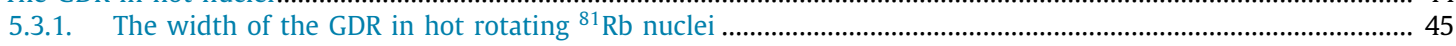

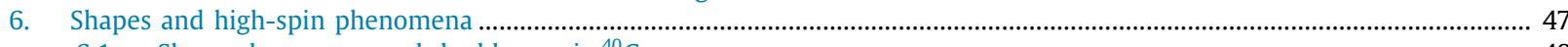

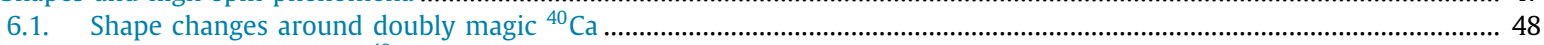

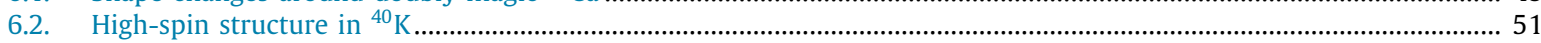

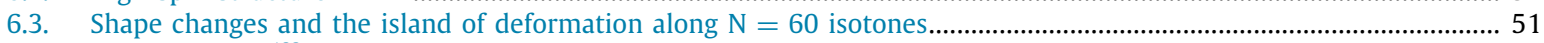

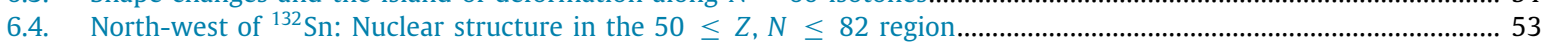

6.5. The gdsh valence space and isomeric states in the $50 \leq Z, N \leq 82$ region.............................................................. 54

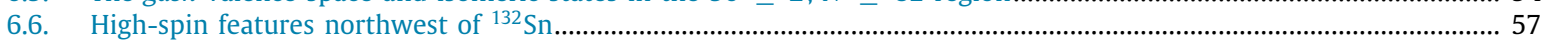

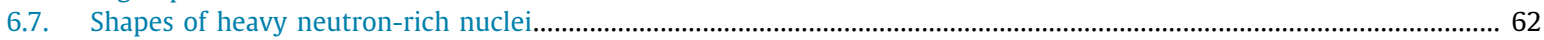

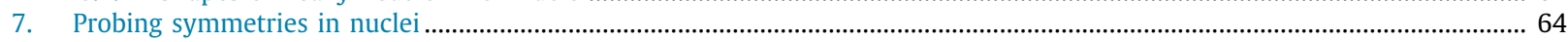

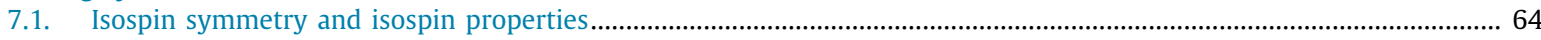

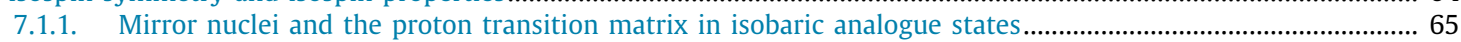

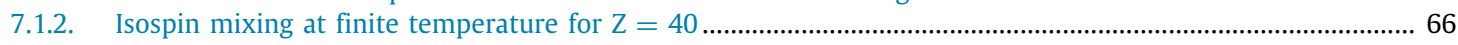

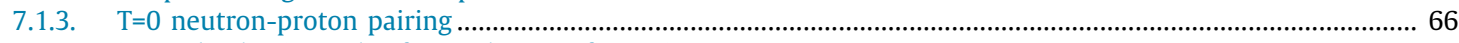

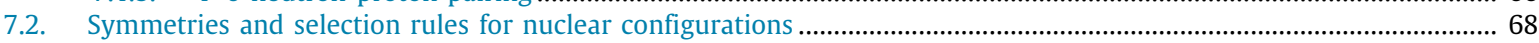

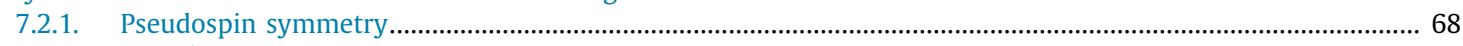

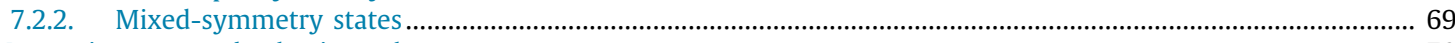

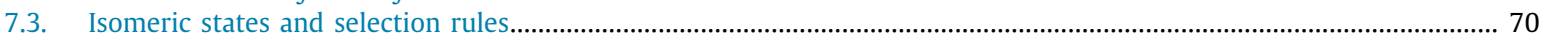

7.3.1. Conservation of $\mathrm{K}$ quantum number from rotational continuum spectra .......................................................... 71

8. The $\gamma$-ray detection to investigate reaction mechanisms and nucleonic degrees of freedom................................................. 73

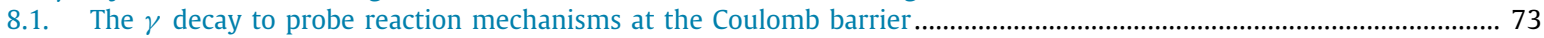

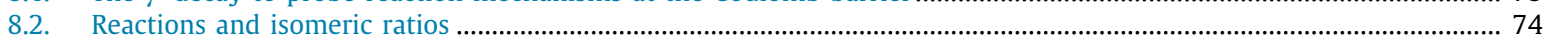

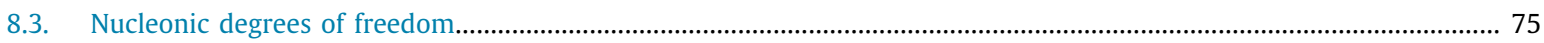

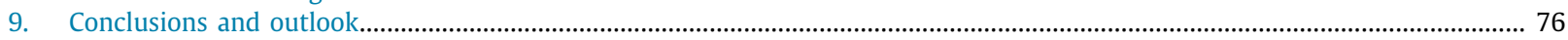

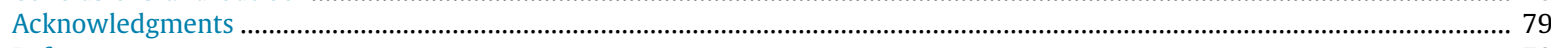

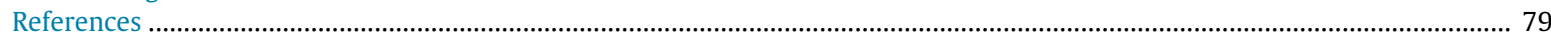

\section{Introduction}

The progress in the understanding of nuclear structure, which manifests itself by excitation modes of various nature, is actually driven by major advances of experimental and theoretical tools. As an experimental tool, high-resolution $\gamma$-ray spectroscopy is of particular importance and plays a decisive role. Already the existing ongoing endeavors demonstrated the capability and versatility of this technique to powerfully address many of the key aspects related to nuclear configurations and interactions. In this review article we focus on the achievements in this context obtained with the AGATA array [1] which is based on the novel $\gamma$-ray tracking technique. Main features of the spectrometer are described in Section 2. Meanwhile an extensive suite of experiments was performed by the AGATA collaboration emanating a number of interesting results. Indeed the experimental efforts address a broad range of topical nuclear physics topics, ranging from studies of shell evolution, single-particle and collective degrees of freedom to the role of three-nucleon forces and of symmetries. Fig. 1 gives an artistic illustration of these topics that were investigated with the high-resolution $\gamma$-ray spectroscopy experiments performed with AGATA. In order to review the new findings we take into account the state of the art emphasizing the progress obtained with the AGATA experiments.

In order to elucidate the various intriguing features of nuclei, in particular when going far away from stability, enhanced and refined detection capability for $\gamma$ rays are needed. This allows spectroscopic investigation of nuclei produced in 


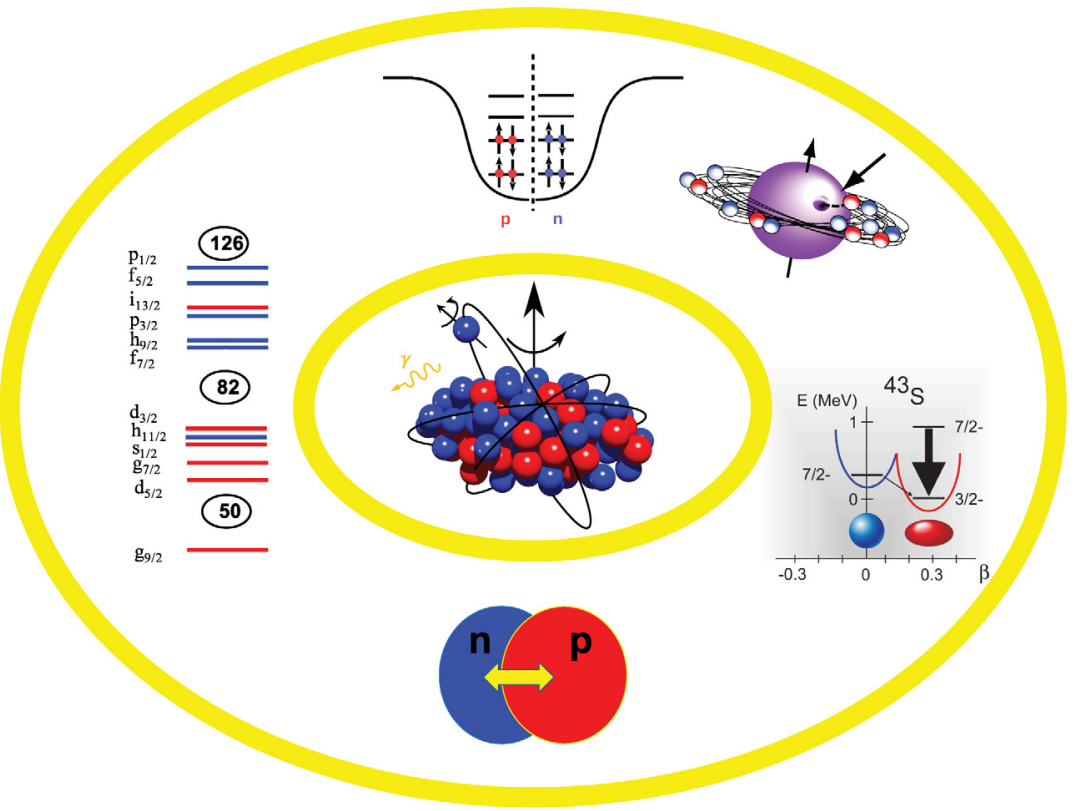

Fig. 1. Artistic view of some of the key properties of the nuclear structure that can be studied using high-resolution $\gamma$-ray spectroscopy. In the center a pictorial representation of the $\gamma$-ray emission from a nucleus rearranging its nuclear configuration. Nuclear properties are schematically depicted here; starting from the left and going clockwise: (i) shell structure and single-particle properties; (ii) level filling with nucleons and the impact of their interactions and symmetries; (iii) complex configurations and isomerism; (iv) nuclear shapes and shape coexistence; (v) collective vibrations of nuclei.

different reactions tuned to selectively populate specific nuclear configurations. Nuclei far from stability are especially interesting for the study and modeling of the different astrophysical nucleosynthesis processes occurring in explosive events in the cosmos.

In the initial Section 2 of this review we first introduce the concept underlying a $\gamma$-ray tracking array to identify the position and energy of the $\gamma$-ray. The evolution of HPGe detectors, the working horses of high resolution $\gamma$-spectroscopy, is briefly discussed up to the highest-performance tracking devices such as AGATA. The specific technical features of the AGATA configurations that were exploited for the series of measurements carried out at the laboratories LNL, GSI and GANIL are presented. The configurations were devised in the best possible way to take advantage of the increasing number of detector modules available at different times. Selectivity and efficacy of AGATA was raised by the coupling to ancillary instrumentation like powerful mass separators and the different selection of available beams at the different accelerators.

For the AGATA experiments low energy stable ion beams were delivered at LNL and GANIL. Rare isotopes produced at low energy with the ISOL technique were available at GANIL while intermediate-energy beams $(v / c \sim 0.5$ and higher) of rare isotopes produced by in-flight projectile fragmentation were employed at GSI.

The measurements executed within the AGATA campaigns at these three laboratories can be classified into six main topics: (i) interactions among nucleons; (ii) single-particle excitations and new shells; (iii) vibrations, collectivity and particle vibration coupling; (iv) nuclear shapes and high-spin phenomena; (v) symmetry and complex excitations; (vi) reaction mechanism studies. The results are here presented and discussed in terms of the advancement in the understanding of interesting open questions within these topics.

It is obvious from the presented arsenal of data that new prospects were opened by AGATA in the three first phases of its implementation and exploitation. Sections 3 to 8 illustrate the AGATA $\gamma$-spectroscopy outcome [2], within the six topics listed above. Comparisons with theory and with data from other arrays, in particular with GRETINA [3] are presented. Two common features emerge from the results discussed in this review: First, the complementarity of the AGATA data with those from other arrays. Second, the availability of several exclusive $\gamma$-spectroscopy data stimulates important theoretical progresses describing nuclear configurations, excitation modes and interactions. It is noteworthy that the obtained results give also a solid ground to challenging future programs as those described in Ref. [4].

As reported in the concluding section, the overarching goals to give answers to unresolved problems and to open new directions for further investigations were achieved with the AGATA experiments made so far. These are important prerequisites which strongly justify the present efforts of the AGATA collaboration to continue the construction of the array up to the final configuration of $4 \pi$ in the coming years. These efforts are expected to permit a great leap forward in addressing uncharted territories of the nuclear landscape. For this purpose the availability of new beams at the major nuclear facilities in the next years is the main essential asset. 


\section{Features of AGATA}

\subsection{AGATA: a $\gamma$-ray tracking spectrometer}

New facilities for the production and acceleration of unstable beams and their experimental perspectives promoted development programs in instrumentation in Europe, in Japan, and in North America. For $\gamma$-ray spectroscopy this came along with the opportunities of the new $\gamma$-ray tracking technique based on advances in highly-segmented positionsensitive HPGe detectors, digital electronics and tremendous progress in data processing. First steps and pioneering developments were advanced by the arrays EXOGAM [5], GRAPE [6], MINIBALL [7], SeGA [8], TIGRESS [9]. These spectrometers were combined with first generation digital electronics and pulse-shape analysis techniques in order to constrain the interaction points of a $\gamma$ ray as it interacts in the detector volume. As a next step the new concept of $\gamma$-ray tracking was elaborated by studying the performance of a $4 \pi$ shell consisting of hundreds of highly segmented positionsensitive Ge detectors [10]. In parallel different detector developments were pursued with the GRETINA prototype at Berkeley [11,12] or the MARS detector at LNL [13].

To identify the energy, time and position of every interaction point of a $\gamma$ ray, as it interacts in the detection volume, this new generation of HPGe detectors was operated with digital electronics and elaborate software tools comprising typically more than thirty signals per detector for high-resolution spectroscopy. Pulse-shape analysis and tracking techniques were exploited to determine how the $\gamma$-ray scatters and is finally absorbed in the $4 \pi$ germanium ball. After identifying the individual interaction energies and positions by pulse shape analysis of all detector signals, the $\gamma$ ray is reconstructed in software by using the characteristics of the photoelectric effect, Compton scattering and the Klein-Nishina formula, giving the differential cross section of photons scattered from a single free electron, as well as pair creation. From Monte Carlo simulations the envisaged tracking array was expected to have a high efficiency due to a maximum coverage of the solid angle with Ge detectors. An excellent ability to correct for Doppler effects is expected by precisely measuring the angle of the emission of the $\gamma$ ray that is determined from the first interaction point in the Ge shell. A very good peak-to-total ratio is achieved by distinguishing between fully and partially absorbed $\gamma$ rays.

As an outcome two $\gamma$-ray tracking arrays were in their first phase of realization in which the proof of principle was elaborated with the AGATA demonstrator and the GRETINA device. These two arrays were the forerunners of the AGATA (Europe) - and GRETA (USA) spectrometers (see Fig. 2).

AGATA is designed to be a $4 \pi \gamma$-ray spectrometer consisting of 180 highly segmented HPGe detectors in its final configuration. It will combine unprecedented efficacy and sensitivity for in-beam $\gamma$-ray spectroscopy [1]. These properties are based on high detection efficiency, excellent position resolution for individual $\gamma$-ray interactions and optimized countrate capabilities. AGATA employs the $\gamma$-ray tracking technique which combines the Pulse Shape Analysis (PSA) technique to obtain the interaction positions of all $\gamma$ rays within HPGe detector volumes of the array and elaborate algorithms to identify the path of the individual $\gamma$ ray [14]. AGATA is a high-resolution spectrometer covering a wide energy range for $\gamma$-rays from a few tenths of $\mathrm{keV}$ to beyond $10 \mathrm{MeV}$.

\subsubsection{Segmented HPGe detectors}

AGATA is based on encapsulated and electrically segmented closed-end coaxial $n$-type high-purity germanium (HPGe) crystals. The crystals have a tapered hexagonal geometry with an asymmetric shape to fit into the $4 \pi$ geometry. This geometry is realized with three different shapes, with a triplet of crystals arranged in identical triple cryostats, such that the full array will consist of 60 cryostats. With this configuration, having a $9 \mathrm{~cm}$ thick germanium shell, a solid angle coverage of up to $82 \%$ is realized. Its rather large inner radius of $22.5 \mathrm{~cm}$ allows the use of ancillary detectors.

The AGATA triple cluster (ATC) detector contains three 36-fold segmented HPGe crystals. The total energy deposited in each crystal is collected in the central contact (core) leading to 37 signals per crystal. One ATC detector comprises 111 high-resolution spectroscopy channels. A picture of the ATC and its cold part is shown in Fig. 3. A detailed description of the ATC detector is given in [15].

All signal channels are equipped with a cold preamplifier stage operated close to the liquid nitrogen temperature of the cryostat. The preamplifiers are characterized by low noise and a large dynamic range for energy detection, pulseshape analysis and timing properties. The reset technique of the core preamplifier allows for an increased counting rate capability of the detectors of more than $50 \mathrm{kHz}$ [16]. The energy range of the ATC detector is substantially extended to 20-180 MeV by a time-over-threshold technique. This is obtained through a precise determination of the reset time [17].

The characterization of the new large volume HPGe detectors included detailed studies of the electron and hole mobility $[18,19]$ and the crosstalk properties of the 36 -fold segmented hexagonal detectors [20]. The results allow for precise crosstalk corrections and an improved energy resolution measured via the segment signals [21]. Moreover, redundancies in segmented high-purity Ge detectors were exploited to increase the energy resolution of these semiconductor devices for detection of electromagnetic radiation in the X-ray and $\gamma$-ray regime. The information of the two electronically decoupled independent measurements, the cathode and the anode electrodes, provides an improved signal-to-noise ratio through a combination of the individually measured signals performed on an event-by-event basis [22]. 

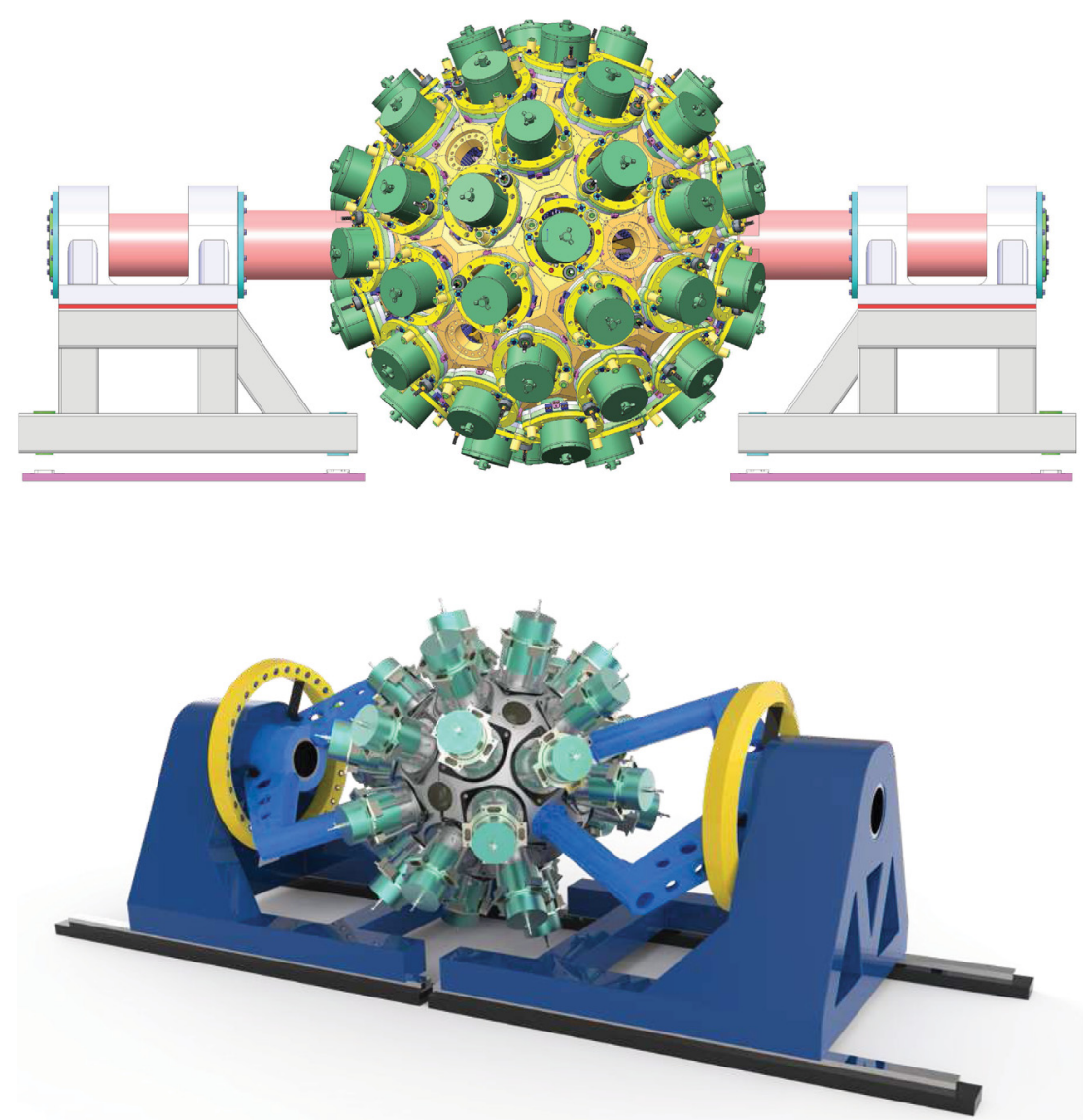

Fig. 2. The future $\gamma$-ray tracking arrays AGATA (top) and GRETA (bottom) are designed to provide nearly $4 \pi$ solid angle coverage. Main difference between the two spectrometers is the number of individual HPGe detectors. AGATA will comprise 180 individual HPGe crystals in 60 cluster cryostat modules, while GRETA will consist of 120 HPGe crystals arranged in 30 modules.

Source: AGATA picture credit: UKRI-STFC; GRETA image reproduce with the permission of the GRETA project at Lawrence Berkeley National Laboratory.

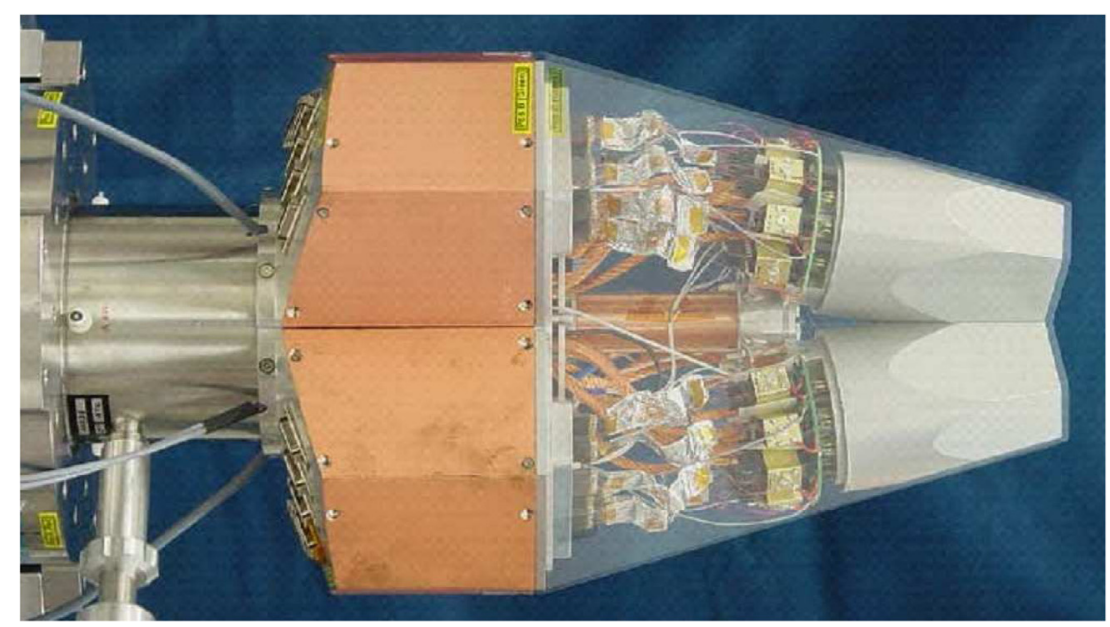

Fig. 3. Picture of the end cap of the AGATA Triple Cluster detector. The copper shielding encloses the preamplifier compartment. The transparent end cap shows the encapsulated detectors which are kept under vacuum. The alignment of the detectors relative to the end cap fulfills tight specifications in order to maximize the solid angle coverage of AGATA. The first feed-back loop of the core and segment preamplifier are mounted directly onto the encapsulated HPGe detectors inside the vacuum. The relevant electronic components are operated at liquid nitrogen temperature to minimize noise contributions. The high signal density inside the cold part of the cryostat comprises 111 signal channels. 


\subsubsection{Front end electronics and data acquisition}

The preamplifier signals are passed to the Front End Electronics (FEE) via mini-D ribbon (MDR) cables. All 37 charge pulse signals of each crystal are digitized synchronously. The signals of one crystal are digitized in one digitizer unit with a sampling rate of $100 \mathrm{MHz}$ using 14-bit analog to digital converter (ADC). A field programmable array (FPGA) of the digitizer implements a fast constant fraction discriminator (CFD) algorithm to generate a fast timing signal needed for the trigger system. The digital data is sent through optical fibers to the preprocessing stage, which is composed of mezzanines that contains the processing logic for 6 segments or the core. The mezzanines are mounted in an Advanced Telecommunications Computing Architecture (ATCA) carrier card. The pre-processing electronics reduces the data from the digitizers by a factor of 100 by selecting only detectors that registered a relevant signal. A moving window deconvolution (MWD) algorithm is used to determine the energy deposited in each detector. The raw trace data, the energy information and the time stamp is then passed to the computer system.

An intense data flow is generated during acquisition by the highly segmented AGATA detectors and the ancillary detectors requesting the Global Trigger and Synchronization (GTS) system, which is contained in another mezzanine. The GTS system provides the central clock (100 MHz digital clock with a 48-bit time stamp) of the spectrometer. It synchronizes the AGATA detectors and the ancillary detectors and distributes a global time stamp to the events passing the system. It is organized in a tree structure where every AGATA detector or an ancillary detector can generate a trigger request. A fully programmable trigger processor validates the incoming requests. Conditions from the trigger system can be implemented into the selection of events at this stage. The ancillary detectors are read out using standard electronics housed in Versa Module Eurocard-bus (VME) crates. The bridge between VME and the AGATA GTS/FEE is a VME module named AGATA VME Adapter (AGAVA) [1].

The processed data is then passed to a computer farm where the PSA, the event building including the ancillary detectors and the tracking is done online and all relevant data for a complete replay of the experiment data is written to disk. After the pre-processing electronics the data is transferred to a computer farm where all the information is stored on hard disk for further data handling. Parallel processing of data flow handling is realized by the Nouvelle Acquisition tempsReel Version Avec Linux NARVAL [23] developed in an object-oriented programming language. A processing network is used to pass data via software packages (called actors) running as individual processes. The processes can run on different computer nodes and the data is distributed over the network. All relevant data were written to disk especially at the beginning of AGATA. Therefore, the experimental information, including the PSA results, can be reconstructed. In this way development and improvement of PSA and Tracking algorithms, calibration of time and energy were pursued in recent years. At a later time during the GANIL phase, the raw data of all individual pulses were replaced by the results of PSA in order to save disk space.

\subsubsection{Pulse shape analysis and $\gamma$-ray tracking}

The AGATA developments of segmented HPGe detectors and their instrumentation were driven by the goal to deduce position information from signals of these semiconductor detectors. The AGATA spectrometer employs PSA to determine the positions of the interaction points with a much higher position resolution than the physical segmentation of the detector volume. Especially the digitization of the detector pulses with high resolution, high bandwidth and high sampling frequency allows to acquire the pulse shapes of the detector signals. This information is exploited to determine the position of charge generation inside the detector volume. In order to localize the scattering sequence following a $\gamma$-ray interaction inside a segmented HPGe detector, the experimental pulse shapes are compared to a basis data set of position dependent pulses. For PSA a sufficient number of pulses have to be provided for typically more than $10^{5}$ basis sites per crystal covering the volume of a large HPGe detector. The reference pulse shapes are collected in lookup libraries. The large dataset from the single crystal are compared to the calculated existing set of signal pulses. This comparison includes the signal of the core electrode, the signal of the hit segment electrode and the signals from the neighboring segment electrodes for every interaction. This is needed because the size and shape of the bipolar transient signals induced in the neighboring segment electrodes contain the important information for the angular and $\mathrm{z}$ position of the interaction. The detailed comparison between reference pulse shapes and measured pulse shapes is the task of PSA algorithms [24] which have to identify the most probable interaction position within the active detector material.

Meanwhile, the two $\gamma$-ray tracking spectrometers AGATA and GRETINA are based on PSA of signals from segmented HPGe detectors to obtain position information on the interaction of $\gamma$ rays. There exist two different procedures to generate the basis set of pulse shapes: it can be obtained from experiment with the existing detectors or the pulses are the result of a calculation which takes all the relevant aspects for a final comparison with measured information into account. To achieve the necessary position resolution of a few millimeters the calculated signal pulses from different interaction points have to be equal to the measured pulses. Therefore the electronic response [16,17] and cross talk effects $[20,21]$ of the electronic has to be incorporated before the comparison between reference and experimental pulses. The procedure how these position dependent detector pulses are generated for the AGATA detectors is given by the AGATA Detector Library described in Ref. [25]. A high precision of the interaction positions is required in order to perform the subsequent $\gamma$-ray tracking with sufficient accuracy. The PSA achieves position resolutions of about $4 \mathrm{~mm}$ FWHM for the AGATA detectors [26-28]

The AGATA HPGe crystals are not surrounded by Compton suppression shields which yields a considerable gain in the solid angle with respect to previous highly efficient $\gamma$-ray spectrometers. Moreover, a good peak-to-total $(\mathrm{P} / \mathrm{T})$ ratio is 


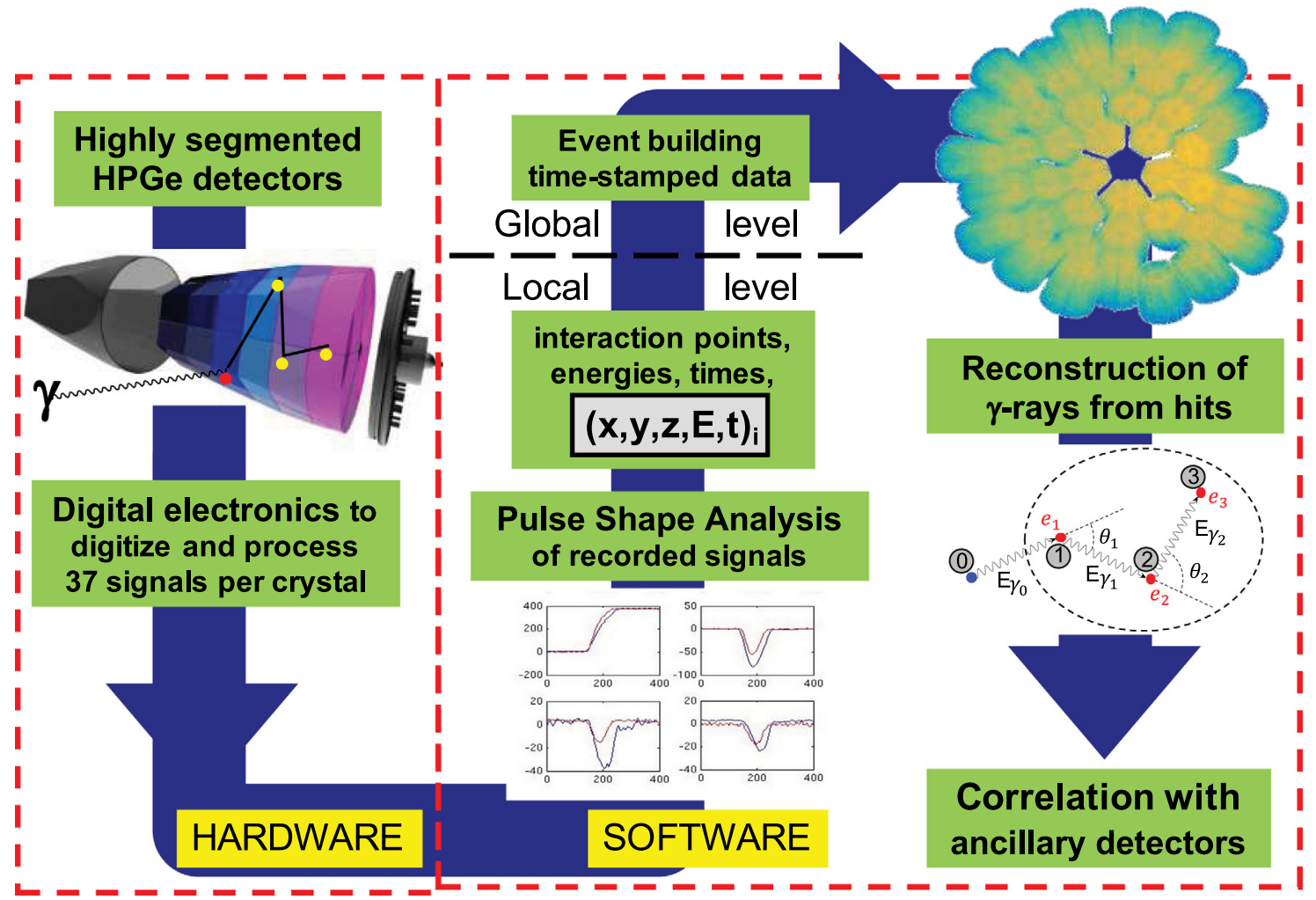

Fig. 4. The principle of $\gamma$-ray tracking is based on few hardware components as highly segmented HPGe detectors, preamplifiers and digital electronics. Digitized information is processed via PSA to obtain position, energy and time information from the recorded charge pulses of the core and 36 segment pulses of each crystal. $\gamma$-ray tracking algorithm assemble first events from the results of all detectors. In a second step the individual $\gamma$-rays and their scattering path across the various individual detectors are deduced from the available position, energy and time information. Especially, the first interaction point is of high interest for in-beam $\gamma$-ray spectroscopy and allows for a high quality Doppler correction. $\gamma$-ray tracking algorithm rely extensively on the identification of Compton scattering across all HPGe detectors. The sensitivity of the spectrometer is enhanced by combining the results with the output of ancillary detectors like light charged particle or neutron detectors close by the $\gamma$-ray detectors or powerful spectrometers to identify heavier reaction products like PRISMA at LNL [29] or VAMOS++ at GANIL [30,31].

achieved by detecting the full energy of photons which scatter from one crystal to another, by means of $\gamma$-ray tracking. The trajectories of the photons as they interact throughout the spectrometer are reconstructed from the accurately determined positions and energies of the individual photon interactions in the HPGe crystals. These results of PSA are then used to reconstruct with best efficiency the photon trajectories which is based on the $\gamma$-ray tracking algorithms (see Fig. 4). The AGATA tracking algorithm disentangles all the interaction points in an event and determines the total energy and emission direction of the photons which have been fully absorbed in the spectrometer. The algorithm used in AGATA is based on clusterisation and forward tracking [14]. For photon energies of interest in the range from tens of keV up to $20 \mathrm{MeV}$, the main physical photon interactions in Ge are Compton scattering, Rayleigh scattering, pair creation, and photo-electric interaction. Compton scattering is the dominant process between 0.150 and $10 \mathrm{MeV}$. The forwardtracking-based algorithm assigns interaction points to clusters from all the HPGe detectors abandoning the individual Ge crystal boundaries. It reconstructs the trajectories of the individual photons employing the properties of the various interactions like the Compton scattering cross section. In this way the energy of the individual $\gamma$-ray, its emission angle from the target and the first and second interaction points are determined. The unique results of $\gamma$-ray tracking yields the energy resolution and high $\mathrm{P} / \mathrm{T}$ value of conventional spectrometers. Increased detection capability is based on the high position resolution in case of $\gamma$-ray emission from fast moving sources in order to cope with Doppler shift and broadening effects especially at relativistic energies [28]. Polarization sensitivity is an inherent property of the AGATA spectrometer deduced from first and second interaction points of the $\gamma$ rays after Compton scattering. This feature was successfully demonstrated for in-beam experiments by [32]. In general, the AGATA tracking performance was measured to be within the specification $[14,33]$.

The number of operational ATCs are expected to grow from the initial demonstrator configuration with five ATCs up to 60 ATCs. The full $4 \pi$ configuration will allow to exploit the full $\gamma$-ray tracking performance. Monte Carlo calculations of low and very high photon multiplicity events of $\mathrm{M}_{\gamma}=1$ and $\mathrm{M}_{\gamma}=30$ yield that the configuration with 180 hexagonal crystals will achieve highest performance figures for detection of $1 \mathrm{MeV}$ photons with a solid angle coverage of $82 \%$ of $4 \pi$, a full-energy efficiency $43 \%(28 \%)$ and a peak-to-total ratio $59 \%$ (43\%) for $\mathrm{M}_{\gamma}=1$ and $\left(\mathrm{M}_{\gamma}=30\right)$ per event, respectively [1]. 

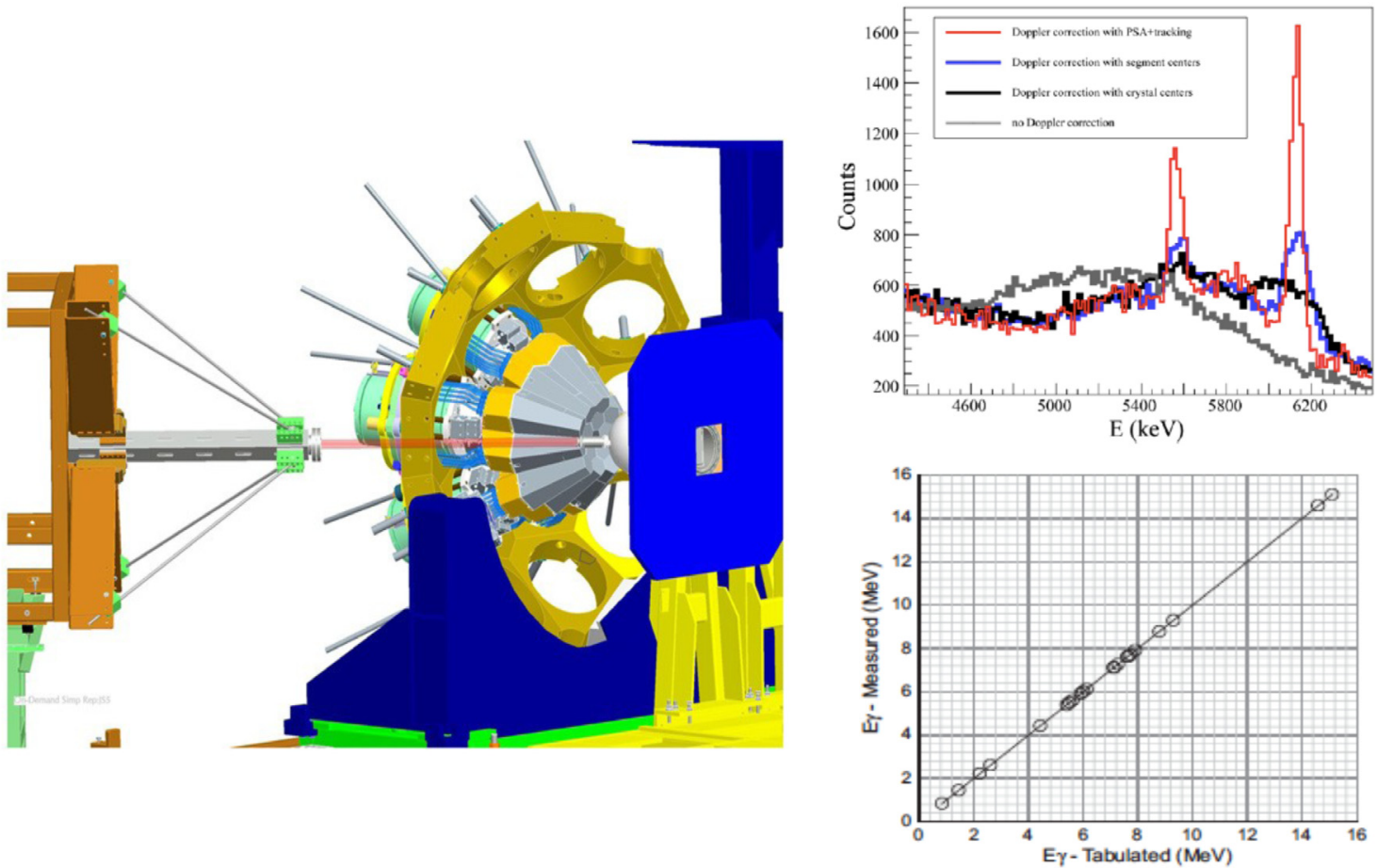

Fig. 5. Left panel: Illustration of the AGATA demonstrator array used at LNL. A telescopic beam line from the accelerator complex enters the AGATA-PRISMA setup from the left side. The five ATCs are mounted inside five flanges of the mechanical support structure, where a subset of 15 flanges covering $1 \pi$ steradian is shown. The spherical reaction chamber is partly visible besides the entrance part of the PRISMA spectrometer (shown in blue). Right top panel: measured spectra including the $\gamma$-decay from the excited state at $6.1 \mathrm{MeV}$ to the ground state in ${ }^{16} \mathrm{O}$. The spectrum shown in the gray color was obtained without applying the Doppler correction, the black (blue) spectrum was obtained using the central electrode (segmentation) position to apply the Doppler correction. The red spectrum used both the segmentation and the pulse shape analysis. Right bottom panel: The measured energy as a function of known transition energies in an interval of few hundreds keV to $15 \mathrm{MeV}$.

Source: Adapted from Refs. [34,35].

\subsection{AGATA at LNL: features and performances}

The first implementation of the AGATA array was realized at the INFN-Laboratori Nazionali di Legnaro, Italy (LNL). A dedicated installation was made to operate the first clusters of HPGe detectors within a set up designed to reach two main goals: the validation of the $\gamma$-tracking concept and the realization of experiments using the heavy-ion beams delivered by the Tandem-ALPI and the PIAVE-ALPI accelerator complex. Five triple clusters of AGATA were mounted on the beam line including the PRISMA [29] magnetic spectrometer. The AGATA array replaced the array CLARA, made of HPGe detectors of clover type [34]. The AGATA set up in combination with PRISMA allowed to perform gamma spectroscopy experiments exploiting grazing reactions as multi-nucleon transfer, or deep-inelastic collisions. In addition, the arrangement permitted also the mounting of ancillary detectors for measurements in which Coulomb excitation, inelastic scattering and fusion-evaporation reactions were employed to populate excited states in nuclei of interest.

The arsenal of ancillary instrumentation that was coupled to AGATA at LNL consisted in the following: (i) the heavyion detector DANTE; (ii) the $\gamma$-ray detector array of $\mathrm{BaF}_{2}$ scintillators, HELENA; (iii) the Cologne plunger for lifetimes measurements of states in nuclei created in grazing reactions; (iv) the highly segmented Si-pad telescope detector TRACE. The description of the main features of these detectors are given in Ref. [34] and they are also described in connection with the results discussed in the next sections.

The overall performances of the AGATA array for the campaign of measurements at the LNL laboratory were very satisfactory and thus a series of successful measurements was made. In view of the physics results, discussed in the following of this paper, it is important to point out how the Doppler correction, needed to construct the $\gamma$-ray spectra, is improved due to the performances of the highly segmented Ge detectors of AGATA and thanks to the application of the tracking algorithms specifically developed for these detectors. In Fig. 5 the effect of the segmentation and tracking on the energy resolution is shown for the detection of $6.1 \mathrm{MeV} \gamma$-rays emitted by a nucleus moving with a velocity corresponding to a v/c value of $20 \%$ (see e.g. [36]). These $\gamma$-rays were produced using the reaction $\left({ }^{17} \mathrm{O},{ }^{16} \mathrm{O} \gamma\right)$ at a bombarding energy of $20 \mathrm{MeV} / \mathrm{A}$ on several targets, namely ${ }^{90} \mathrm{Zr},{ }^{124} \mathrm{Sn},{ }^{208} \mathrm{~Pb}$ and ${ }^{140} \mathrm{Ce}$. These data are a byproduct of the experiments whose physics results are presented and discussed in Section 5.2. One can note a clear improvement in the energy resolution, dominated by the Doppler broadening, by going to determination of the interaction position from the central electrode 

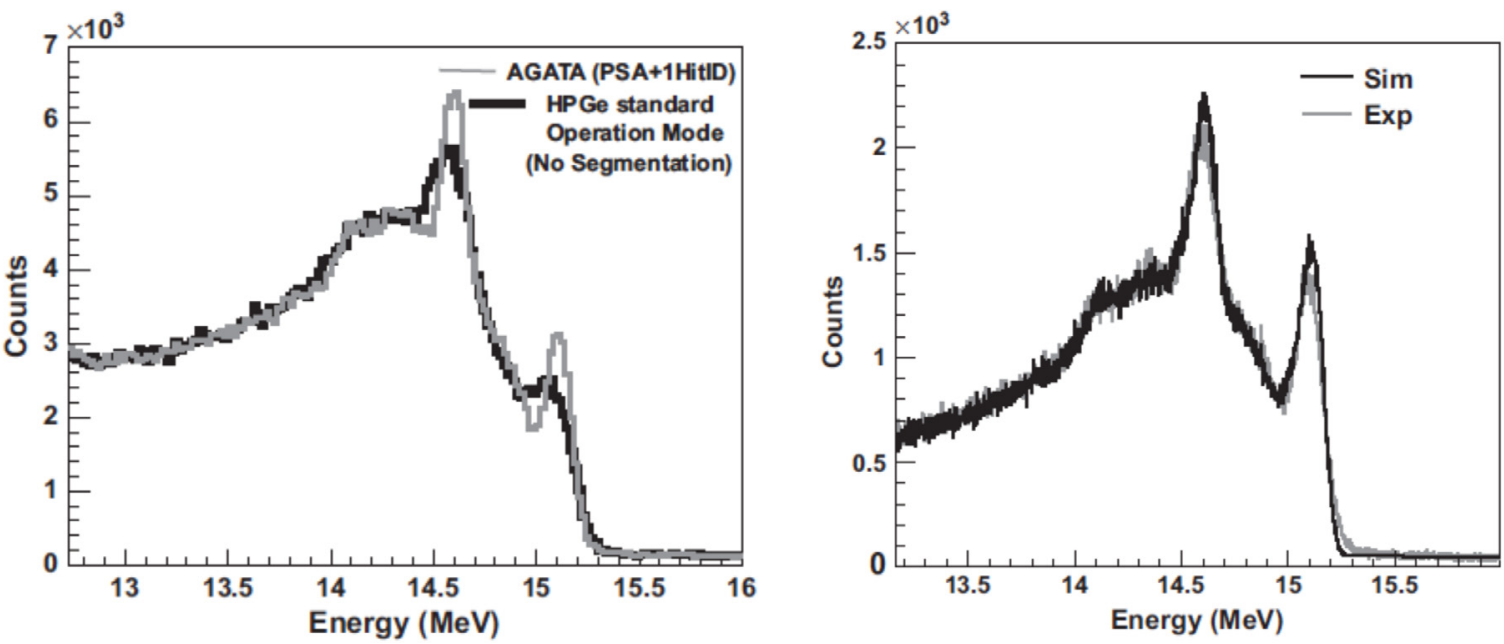

Fig. 6. In both panels spectra corresponding to the detection the $\gamma$-rays of energy 15.1 MeV produced using the reaction ${ }^{2} \mathrm{H}\left({ }^{11} \mathrm{~B}, \mathrm{n} \gamma\right)^{12} \mathrm{C}$ at $\mathrm{E}_{\text {beam }}=19.1 \mathrm{MeV}$ are shown. Left panel: the black spectrum is obtained without using the segmentation features while the gray spectrum was obtained by using the segmentation and applying the PSA. Right panel: The measured spectrum obtained applying the PSA in comparison with the corresponding simulation of interactions in the detector.

Source: Adapted from Ref. [35].

to that from the segment with the largest energy deposition. When both the segmentation and tracking algorithms are used one obtains the best possible resolution (see the red spectrum in the top-right panel of Fig. 5).

With the AGATA setup at LNL the response of the HPGe detectors to $\gamma$-rays in the energy interval 2-15 MeV was measured [35]. Gamma rays with energies between 2 and $9 \mathrm{MeV}$ were produced using an Am-Be-Fe radioactive source. This is a composite source in which the $\alpha$ decay from the Am nucleus is exploited to induce an $(\alpha, \mathrm{n})$ reaction on the Be nucleus. The neutron emitted from this reaction are used to induce neutron capture on a Fe slab and the several $\gamma$-rays following neutron capture are in a wide energy interval from few hundreds $\mathrm{keV}$ to $9.3 \mathrm{MeV}$. With the data from this source several information were obtained for the HPGe detectors of AGATA. The linearity of detectors and electronics over a wide energy interval, namely the energy-to-pulse-height conversion, was excellent and it resulted to be linear within $0.05 \%$, as one can see in the right-bottom panel of Fig. 5 . In segmented detectors where the energy release in the different segments have to be added, a good linearity is an important requirement. The multiplicity of the different interactions undergone by the incident $\gamma$-rays to deposit the full energy was extracted from these Am-Be-Fe data using the segmentation information. The distributions of multiplicity for different $\gamma$-ray energies were also simulated using the GEANT4 code. An important result was found in this connection, namely that the application of gamma-ray tracking allows a suppression of background radiation caused by n-capture in Ge nuclei. Since the used Am-Be-Fe radioactive source provided a large number of $\gamma$-lines, it was possible to obtain the evolution of the energy resolution over the wide 1-10 MeV interval, which was found to follow very well the $\mathrm{E}^{-1 / 2}$ behavior. In particular, for the line at highest energy from the radioactive source, at $9297.8 \mathrm{keV}$, a resolution of $6.1 \mathrm{keV}$ (FWHM) is found using the developed algorithms. A simple add-back method yields a resolution of $7.6 \mathrm{keV}$ (FWHM).

High energy $\gamma$-rays were produced using the reaction ${ }^{2} \mathrm{H}\left({ }^{11} \mathrm{~B}, \mathrm{n} \gamma\right)^{12} \mathrm{C}$ at $\mathrm{E}_{\text {beam }}=19.1 \mathrm{MeV}$. The measured spectra in the energy region up to $15.1 \mathrm{MeV}$ are shown in Fig. 6 . The left panel shows how the energy resolution improves by using the segmentation and applying the PSA as compared to the case in which only the segmentation is used. In the right panel the same higher resolution spectrum of the left panel is compared with the corresponding GEANT simulation, which includes the distribution of the direction of the velocity of the emitting ${ }^{12} \mathrm{C}$ nucleus. It is found that the resolution of $116 \mathrm{keV}$ is due to the remaining Doppler broadening. Indeed it is much larger than the $10 \mathrm{keV}$ value expected for the intrinsic resolution as deduced from radioactive source data. For this measurement the velocity vector of the recoiling ${ }^{12} \mathrm{C}$ nucleus could not be measured and thus it was not possible to obtain directly the intrinsic resolution of the $\gamma$-rays at $15.1 \mathrm{MeV}$.

In the next sections, in connection with the results on nuclear structure, it appears clear that the LNL setup was particularly well suited to study nuclei populated with multi-nucleon transfer reactions due to the additional information provided by the spectrometer PRISMA. Furthermore, the setup in combination with other ancillary instrumentation was also very well suited to study excited nuclear states of various nature populated with inelastic collisions and to access hot rotating nuclei populated with fusion reactions. Investigations to obtain more insight in the reaction processes around the fusion barrier were also performed by using gamma spectroscopy as a unique tool.

Multinucleon transfer reactions with the AGATA-PRISMA set up. As discussed in the following sections of this review, Multi-Nucleon Transfer (MNT) reactions were largely employed in experiments performed with AGATA at the laboratory LNL to populate moderate neutron-rich nuclei and to study their structure via $\gamma$-ray decay. An important asset in this 
connection is the understanding of the population cross section when heavy ion beams and heavy target are employed. The capability of the setup to perform X-ray and $\gamma$-ray spectroscopy turned out to be crucial at the presence of many different reaction channels that could create unwanted background. Therefore the complete and detailed detection of all reaction products both of projectile and target like types, of the separation of fission products, and of the determination of the total kinetic energy loss were performed for the ${ }^{136} \mathrm{Xe}^{238} \mathrm{U}$ reaction at a bombarding energy of $1 \mathrm{GeV}$ [37]. This reaction is particularly challenging for gamma-ray spectroscopy because of the presence of many nuclei as fission products from the target in addition to the fewer ones related to the MNT process. On the other hand, it offers the possibility to address a region of moderate neutron-rich nuclei for which nuclear structure information is needed. It is known that for the combination of the most neutron-rich stable $U$ isotopes as target material and the heavy neutron-rich ${ }^{136} \mathrm{Xe}$ beam the cross section of multi-nucleon transfer reactions depend on the respective $Q$ value. With neutron deficient stable beams, only neutron pickup and proton stripping channels are available. Instead, with neutron-rich beams, also neutron stripping and proton-pickup channels become available, leading to the possibility to populate neutron-rich heavy nuclei [38].

A schematic view of the setup, comprising the AGATA, DANTE and PRISMA detector systems, is presented in the top part of Fig. 7. The DANTE array consists of an ensemble of position sensitive multichannel plate detectors placed at angles such to measure the target like reaction products [39]. The projectile like reaction products were detected by the PRISMA spectrometer while X-and $\gamma$-rays were measured in the AGATA array. This setup provides all the necessary information for complete ion identification. An event-by-event trajectory reconstruction algorithm uses the entrance angle, the position at the focal plane, and the properties of the magnetic fields to calculate the trajectory length $\mathrm{L}(\theta, \Phi)$ of the ions and the curvature radius $\mathrm{R}$ inside the dipole magnet. The $\mathrm{L}(\theta, \Phi)$ information in combination with the time-of-flight measurements yields the velocity vector of reaction products entering PRISMA.

The different values of nuclear charge $Z$ of the measured nuclei were selected by applying cuts in the energy loss matrix shown in the top-right panel of Fig. 7. The findings for the $Z$ distribution (see the bottom-right panel of Fig. 7) is characterized by yields caused by the presence of both fission and MNT reaction mechanisms. Thus it is important to separate the two contributions and for this purpose the matrix nuclear charge $\mathrm{Z}$ versus time of flight (ToF) was used. This matrix and 4 projections on the ToF axis for different values of $Z$ are shown in central panel of Fig. 7 . One can see clearly the two contributions which are rather well separated. With this additional condition it was possible to obtain $\gamma$-ray spectra corresponding to MNT and fission events. An example is in the left panel of Fig. 7 showing two spectra for ${ }^{238} \mathrm{U}$, one corresponding to MNT events and the other, having a much lower yield, corresponding to fission events. The ${ }^{238} \mathrm{U}$ nucleus was identified through a coincidence with the ${ }^{136} \mathrm{Xe}$ ions and by applying the Doppler correction using the velocity of nuclei exiting the target.

The measurement of the target like particle, being in this case ions in the actinide region, was achieved by applying kinematic coincidences between the binary reaction products, i.e., beam like and target like nuclei. Unfortunately, the corresponding position information of the DANTE detectors could not be resolved and thus only the time information was used. In addition, different observables provided by the AGATA array were employed. The signatures of surviving actinide nuclei were the observation of the characteristic X rays, of the neutron-induced $\gamma$-rays, and, the detection of $\gamma$-rays. Portions of tracked $\gamma$-ray spectra in the X-ray region are shown in the left and central panels of Fig. 8 . They were obtained with conditions on the transfer like $\Delta$ ToF peak (with $\Delta$ ToF being the difference from ToF's from PRISMA and DANTE) and with Doppler correction for different actinide recoils ranging from $\mathrm{Ac}(\mathrm{Z}=89)$ to $\mathrm{Pu}(\mathrm{Z}=94)$. Using the $\mathrm{X}$-ray yields, the distribution of population of actinides, characterized by their $\mathrm{Z}$ and normalized to the Pu channel, was obtained. This is shown in the right panel of Fig. 8 in comparison with data from $\Delta$ ToF and predictions obtained with the GRAZING code, based on the model of [38]. While in the case of $Z=90$ the model fails in reproducing the data, for $Z=$ 91-94 a good account of the data is observed.

The experimental cross section as a function of mass was deduced for different proton pick-up and stripping channels and it was normalized to the calculated cross section of the $+1 \mathrm{n}$ channel ${ }^{137} \mathrm{Xe}$. It is shown in the central and right panels of Fig. 9 for the $0,+p,+2 p,-p$, and $-2 p$ channels before and after correction for the response function and in comparison with the corresponding predictions based on the model of [38]. Not always a good agreement is found and in particular the $+2 \mathrm{p}$ channel is much underestimated by the predictions.

A further inspection of the reaction features was made by deducing the trend of the neutron yield in different reaction channels. Indeed particle evaporation strongly affects the final yield distribution of both binary partners and it hinders the production of very neutron-rich nuclei. Neutron yields were experimentally deduced from the gamma-rays visible in the AGATA spectra corresponding to the $\left(n, n^{\prime} \gamma\right)$ reactions on ${ }^{27} \mathrm{Al},{ }^{70} \mathrm{Ge},{ }^{72} \mathrm{Ge}$, and ${ }^{74} \mathrm{Ge}[40,41]$. In the time spectrum, these hits are delayed due to the longer time-of-flight of the neutrons. The fast-moving neutrons scatter and interact with the ${ }^{74} \mathrm{Ge}$ of the AGATA detector material by exciting the first $2^{+}$state (with a transition of $596 \mathrm{keV}$, very visible in the spectra) via the ${ }^{74} \mathrm{Ge}\left(\mathrm{n}, \mathrm{n}^{\prime} \gamma\right)$ reaction. This yield relative to that of the $511 \mathrm{keV}$ line (not depending on neutron evaporation) is shown in the left panel of Fig. 9 for different Xe isotopes (both the for fission and transfer contributions). After neutron transfer, primary products may be highly excited due to the effects of large energy loss, in turn leading to particle (mainly neutron) evaporation. As expected, the transfer channels after neutron stripping show more neutron interactions in the Ge detectors (red points in the left panel of Fig. 9). With increasing number of neutrons which are transferred to the target nucleus, more evaporated neutrons are detected. Fission fragments (blue points in the left panel of Fig. 9) are related to much more neutron induced background since several neutrons are produced in each fission process.

The detailed analysis of data from the measurement of the ${ }^{136} \mathrm{Xe}^{238} \mathrm{U}$ reaction at a bombarding energy of 1 GeV [37] demonstrates the high potential for the use of the high efficiency $\gamma$-ray tracking spectrometer AGATA in combination 
(i)

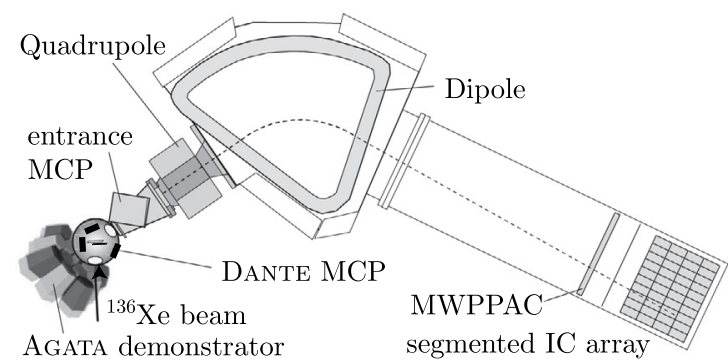

(iii)

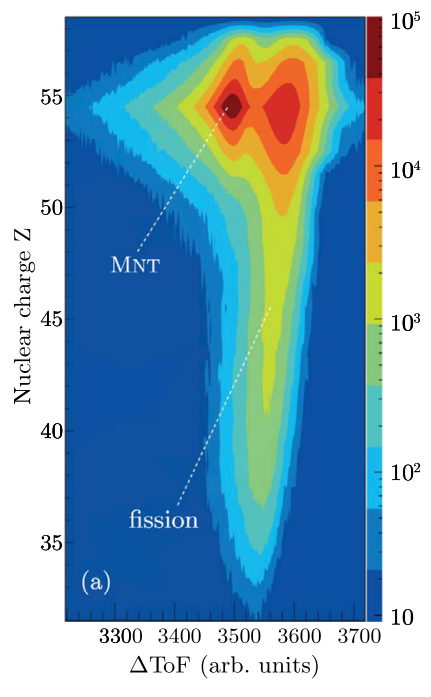

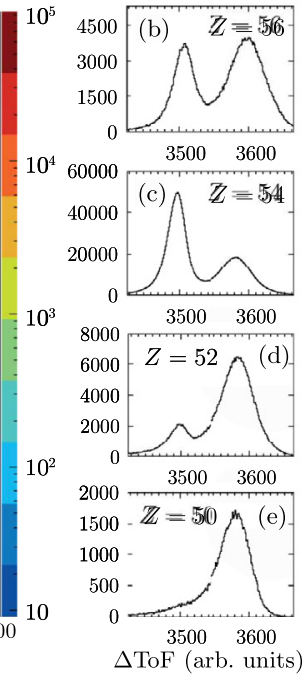

(ii)

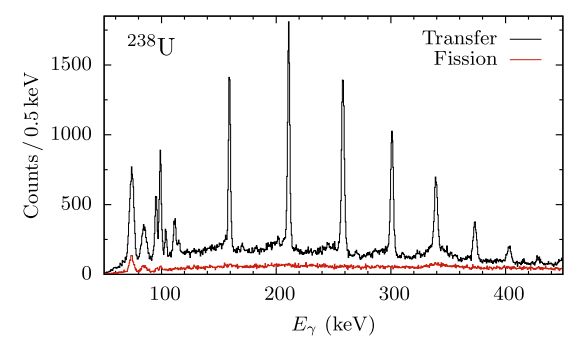

(iv)
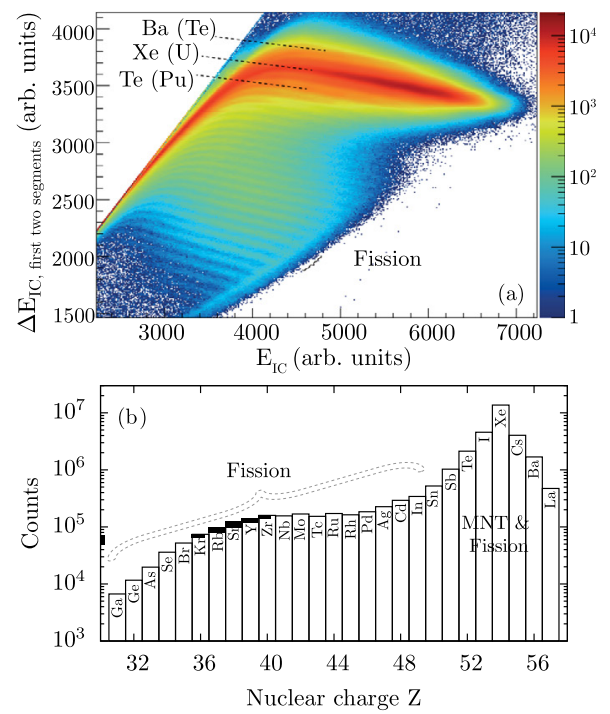

Fig. 7. (i): Sketch of the experimental setup comprising the $\gamma$-ray spectrometer AGATA, the ejectile-detecting heavy-ion mass spectrometer PRISMA, and the particle detector DANTE (not to scale). The DANTE MCP system in the scattering chamber covers the grazing angles of the binary reaction products. (ii): Recoil Doppler-corrected spectrum of ${ }^{238} \mathrm{U}$ with transfer and fission-like $\Delta$ ToF gates. A gate on the right part of the fission-like $\Delta$ ToF peak yields a flat $\gamma$-ray background spectrum (red). The spectrum gated by the left part of the $\Delta$ ToF distribution shows distinct known peaks from the ${ }^{238} \mathrm{U}$ rotational band up to spin $22 \mathrm{~h}$. (iii): (a) The Time-of-flight $\Delta$ ToF difference spectrum between PRISMA and DANTE is plotted against the nuclear charge $Z$. In the region near the Xe nuclei the left maximum of the distribution is caused by multinucleon transfer products; these are marked by MNT. The right part of the distribution is caused by fission products and is clearly separated from transfer products. The fission fragments cover a broad $\mathrm{Z}$ range and show for lower $\mathrm{Z}$ values below $\mathrm{Z}=50$ one distinct $\Delta \mathrm{ToF}$ peak. Selected $\Delta \mathrm{ToF}$ projections for (b) Ba, (c) Xe, (d) Te, and (e) Sn nuclei are shown at the right side. (iv): (a) energy loss matrix of the Ionization Chamber (IC) in the focal plane of the PRISMA spectrometer, giving the energy deposited in the first two layers versus the total deposited energy. (b) Yield distribution of nuclear charges from $G a(Z=31)$ to La $(Z=57)$. The broad structure around $Z=42$ results from asymmetric actinide fission residues. Corresponding fission partners in the $X e$ region overlap with the multi-nucleon transfer products in the vicinity of ${ }_{54}^{136} \mathrm{Xe}$.

Source: Adapted from Ref. [37].

with the mass spectrometer PRISMA and the ancillary multichannel-plate detector DANTE. It shows that the setup was able to track down the elusive $\gamma$ rays from weakly populated submillibarn reaction channels under demanding conditions caused by the high fission background from the employed actinide target.

\subsection{AGATA at GSI: features and performances}

The AGATA array was installed in 2012-2014 at GSI (Darmstadt, Germany). The GSI accelerators coupled with FRS are a fragmentation facility delivering radioactive beams produced in relativistic energy fragmentation and in-flight fission. The ions of interest are selected and identified on an event-by-event basis using the Fragment Separator (FRS) [42]. Each individual ion from fragmentation or fission is identified based on time-of-flight, particle tracking and energy loss measurements. The fast moving radioactive ions impinge on a secondary target positioned at the final focal plane of the FRS. The secondary reaction products are identified by means of energy loss, total energy and time-of-flight measurements 

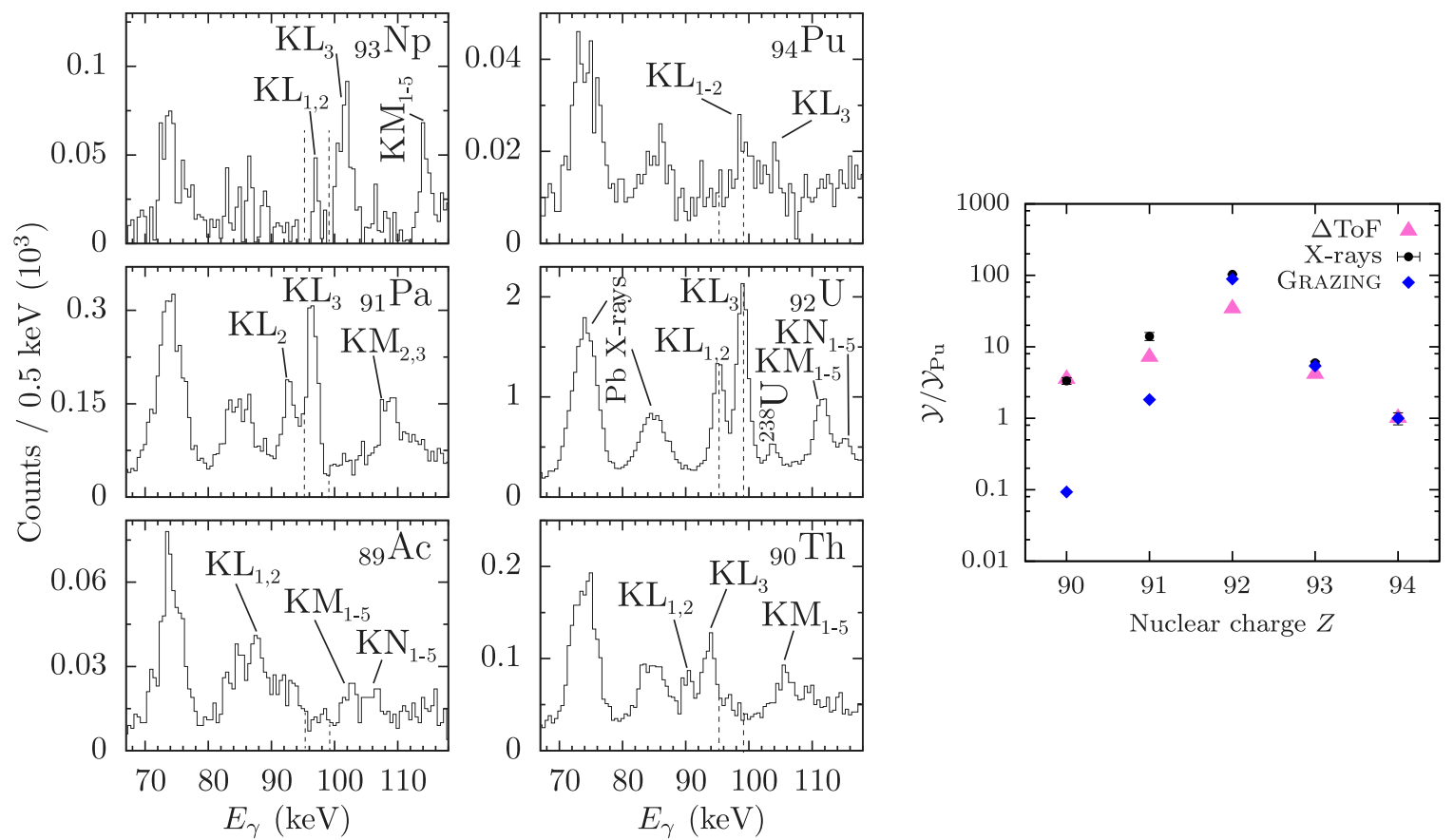

Fig. 8. Left panels: Tracked singles $\gamma$-ray spectra obtained with a gate on the transfer-like $\Delta$ ToF peak, Doppler-corrected for different actinide recoils ranging from $\mathrm{Ac}(\mathrm{Z}=89)$ to $\mathrm{Pu}(\mathrm{Z}=94)$ showing $\mathrm{X}$-ray emission. ${ }^{92} \mathrm{U} \mathrm{KL} \mathrm{X}$-rays are indicated with dashed lines. The Pa (corresponding to $\mathrm{Z}=55$ Cs ejectiles) and $\mathrm{Np}$ (corresponding to $\mathrm{Z}=53 \mathrm{I}$ ) channels are corrected for $\mathrm{U}$ contamination. The Pu channel corresponding to ${ }^{52} \mathrm{Te}$ ejectiles is only weakly populated. Right panel: (Color online) Mass-integrated yields of actinide nuclei from to their X-ray yields in the recoil Doppler corrected $\gamma$-ray spectra (black points) and from the PRISMA-DANTE $\triangle$ ToF yield scaled to the X-ray distribution (magenta triangles) together with cross sections calculated by the GRAZING code (blue rhomboids). All distributions are normalized to the Pu channel.

Source: Adapted from Ref. [37].
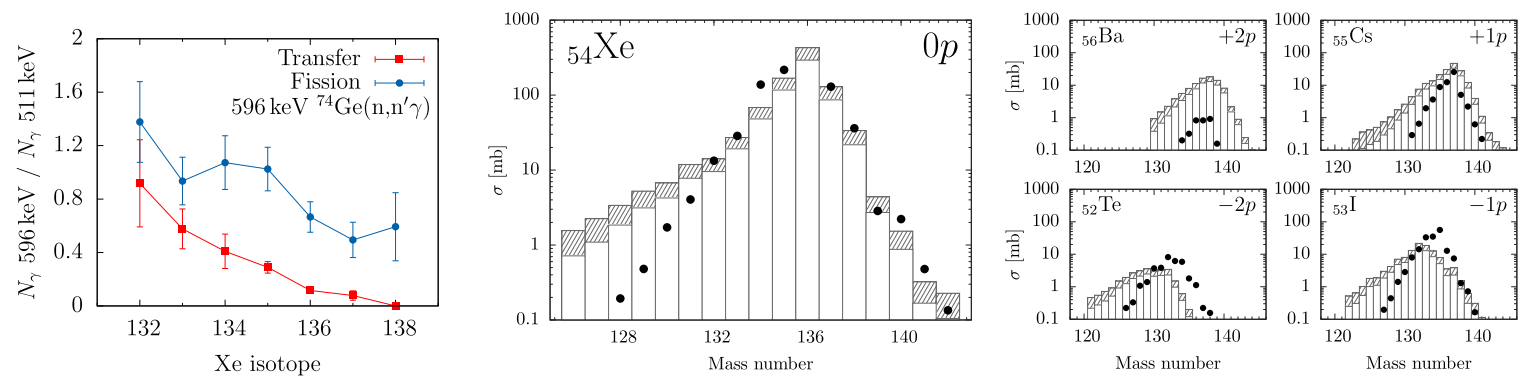

Fig. 9. Left panel: $\gamma$-ray intensities of neutron-induced background radiation with gates on the transfer- and fission-like $\Delta$ ToF peak. In the channels in which neutrons were stripped from the ejectile nucleus, more neutrons react with the surrounding HPGe detectors, hindering the production of actinide binary partners. Central panel and right panels: Cross sections of a GRAZING calculation (points) and experimental yields (histogram bars) normalized to the calculated cross section of the $+1 \mathrm{n}$ channel ${ }^{137} \mathrm{Xe}$. The mass yields are corrected for Xe contamination in the I and Cs channels. Fission events are excluded by a cut in the time-difference spectra between PRISMA and DANTE. Gray-shaded bars indicate the efficiency correction by applying the PRISMA response function to the experimental data.

Source: Adapted from Ref. [37].

employing the Lund York Cologne CAlorimeter (LYCCA) [43]. The main components of the full setup are presented in Fig. 10, while the photograph of AGATA at the final focal plane of FRS is shown in Fig. 11. In this picture the HECTOR+ array consisting of large volume $\mathrm{LaBr}_{3}$ detectors [44] is also visible.

The AGATA experiments at GSI evolved from and were built on the expertise obtained in previous campaigns using fast beams. The fast-RISING campaign [45] in 2003-2005 used sevenfold EUROBALL Cluster detectors which were formerly part of the EUROBALL array, and additional MINIBALL [7] detectors mounted closer to $90^{\circ}$. The direct precursor of the AGATA campaign was the more recent PRESPEC campaign which run in 2010-2011 [46,47]; all sub-systems, with the exception of the high-resolution HPGe array, were identical with those used in the AGATA campaign.

Fragmentation facilities, such as GSI, can produce beams of any element lighter than the projectile. Their lifetimes can be also rather short since the typical flight-time between primary target where the radioactive ions are produced 


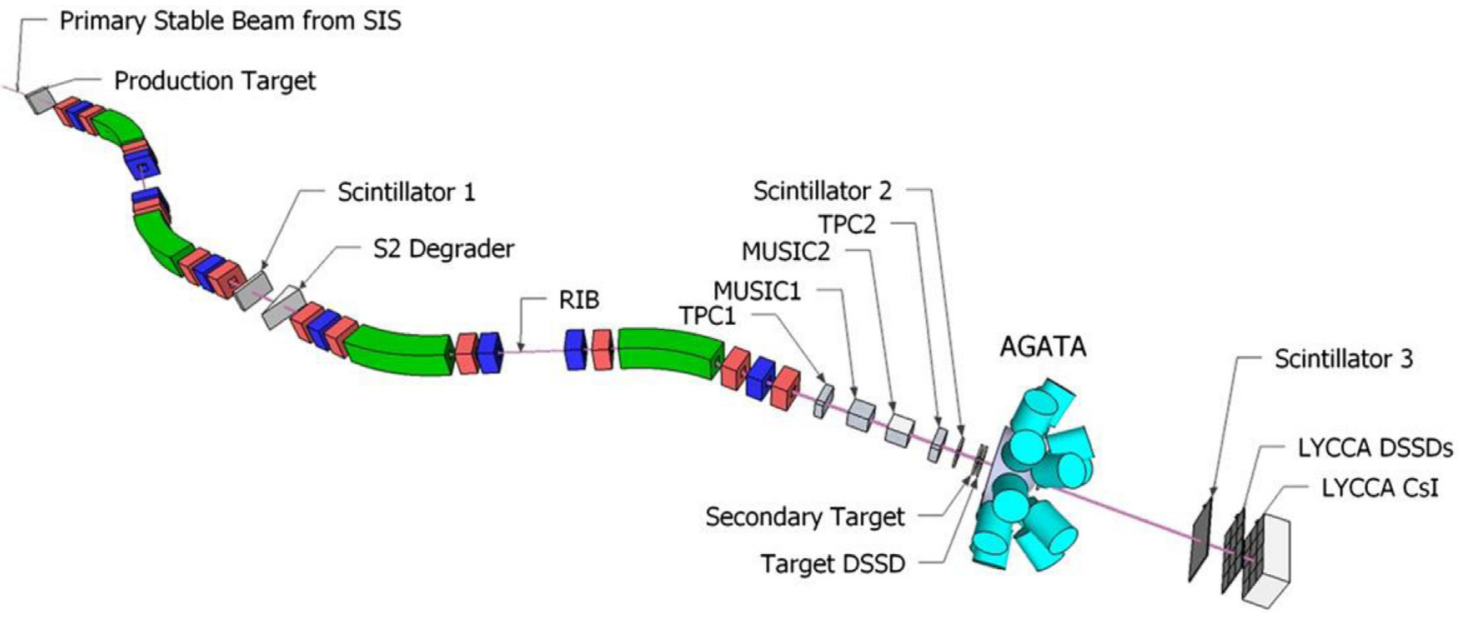

Fig. 10. The AGATA array placed at the final focal plane of the fragment separator at GSI. The detectors needed to track and identify both incoming and outgoing ions are also shown. Source: Figure taken from [48].

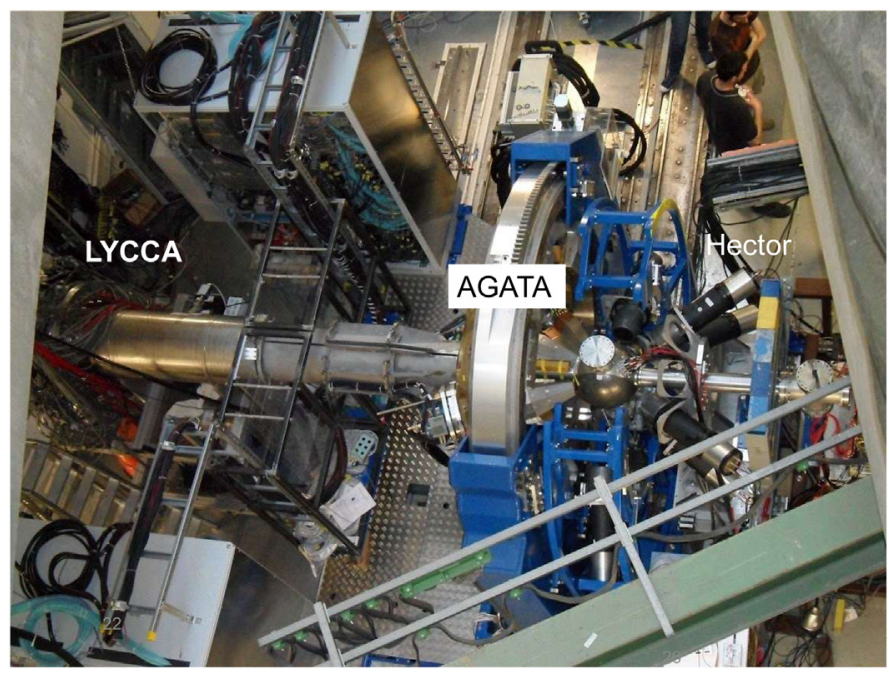

Fig. 11. Photograph of the AGATA array at the final focal plane of the Fragment Separator at GSI. The LYCCA array [43] used for reaction product identification and the HECTOR array consisting of large volume $\mathrm{LaBr}_{3}$ detectors [44] are also indicated.

and the secondary target where they are employed in reactions (through the FRS) is $\sim 300 \mathrm{~ns}$. Among fragmentation facilities, GSI is unique in being able to provide high primary-beam energies up to $\mathrm{E} / \mathrm{A} \sim 1 \mathrm{GeV}$. The high energy ensures that the fragmentation products emerge from the production target bare, without electrons, even for the heaviest (below uranium) elements. The well-defined charge is essential for the unambiguous ion-by-ion identification. The drawback of fragmentation facilities is the secondary beam quality, manifesting itself in large beam sizes and wide energy distribution. At GSI, when using the FRS in achromatic mode (smallest beam size), typical beam characteristics are $\Delta \mathrm{x}=\sim 1-3 \mathrm{~cm}$ (FWHM), $\Delta \mathrm{y}=\sim 1 \mathrm{~cm}$ (FWHM) and $\Delta \mathrm{E} / \mathrm{A}=\sim 7 \mathrm{MeV}$ (FWHM) (for a ${ }^{202} \mathrm{~Pb}$ beam at $\mathrm{E} / \mathrm{A}=158 \mathrm{MeV}$ ).

The experiments are typically performed at beam energies on the secondary target of $E / A=50-200 \mathrm{MeV}$. This is determined by a range of factors such as radiation background (increasing with beam energy and with Z), target thickness (the high beam energy is an advantage which gives us a way to use thicker targets) and need of identification (faster beams are easier to track and identify). The radiation background has both atomic and nuclear components, both decreasing exponentially with increasing photon energy. The atomic background depends strongly on both the $\mathrm{Z}$ of projectile and target and it dominates at low energies [49], typically below $200 \mathrm{keV}$, while the nuclear one dominates at higher energies.

Experiments using high energy beams have their challenges and opportunities. The Lorentz boost leads to a $\gamma$-ray intensity distribution which is peaked at forward angles in the laboratory frame. Consequently, the AGATA array is placed downstream of the secondary target. The $\gamma$-rays emitted by moving nuclei at relativistic energies are extremely Doppler 


\section{Table 1}

Requirements for the beam tracking at different velocities. The quoted values correspond to an increase of $10 \%$ in the FWHM of the $\gamma$ peak, with respect to the FWHM calculated assuming a perfect knowledge of the kinematics.

\begin{tabular}{llll}
\hline Uncertainty $(\sigma)$ on: & $\beta=5 \%$ & $\beta=20 \%$ & $\beta=50 \%$ \\
\hline Source position $(\mathrm{cm})$ & 1.5 & 0.5 & 0.3 \\
Direction (degrees) & 2 & 0.6 & 0.3 \\
Velocity magnitude $(\%)$ & 2.4 & 0.7 & 0.3 \\
\hline
\end{tabular}

shifted, and the $\gamma$-ray energies in the center-of-mass projectile frame have to be reconstructed. At small forward angles the Doppler shift results in the need to detect much higher $\gamma$-ray energies $\left(E_{\gamma}\right)$ than that emitted in the rest frame $E_{\gamma 0}$. The quality of the Doppler correction depends on the intrinsic resolution of the detectors and the uncertainty on the knowledge of both the angle $\theta$ and velocity $\beta:\left(\Delta E_{\gamma 0}\right)^{2}=\left(\frac{\partial E_{\gamma 0}}{\partial E_{\gamma}} \Delta E_{\gamma}\right)^{2}+\left(\frac{\partial E_{\gamma 0}}{\partial \beta} \Delta \beta\right)^{2}+\left(\frac{\partial E_{\gamma 0}}{\partial \theta} \Delta \theta\right)^{2}$.

The impact of the uncertainties in the emission position, and velocity vector on the $\gamma$-ray energy resolution was addressed in the full AGATA simulations presented in Ref. [50]. The energy resolution (FWHM) is given for the different $\beta$ values as function of uncertainties in velocity and recoil direction in Fig. 12. Note that even assuming a complete knowledge of $\theta$ and $\beta$ the resolution is much worse for fast moving ions. Table 1 lists the requirements for beam tracking in order to limit the increase of the energy resolution to $10 \%$.

The velocity of the projectile is measured both before and after the secondary target using the FRS and LYCCA detectors, respectively (see the scintillators used for time-of-flight in Fig. 10). These can be used to infer the actual value of the speed of the projectile when the $\gamma$ ray is emitted, which however depends on factors like the target thickness and lifetime of the excited states. The $\theta$ emission angle calculations rely on the tracking of particles and the determination of the position of the first interaction point in AGATA.

Simulation of the performance of the AGATA array specifically for the GSI conditions were performed in [48]. Full energy efficiency, energy resolution and peak-to-total ratio were calculated for different target-array distances. Different geometries, involving both traditional triple-crystal detectors and double-crystal detectors were considered. Since the size of the fragmentation beam is much larger than the size of a single crystal, in forward direction the dimensions of the beam pipe (to let the reaction products exit to LYCCA) were optimized by using double crystal detectors. Lifetime measurement capabilities based on lineshape analysis were also scrutinized.

The AGATA array at GSI consisted of six triple and two double detectors, numbering in total 22 crystals. The array was placed at the nominal distance of $23.5 \mathrm{~cm}$ from the center of the secondary target. The array provided optimal energy resolution and improved efficiency compared to the previous fast-RISING and PRESPEC campaigns. However, with only $60 \%$ of detector surfaces in contact with neighboring crystals, the tracking capability was reduced accordingly. The measured performance of the AGATA array using radioactive sources at GSI is summarized in [28]. For the $1173 \mathrm{keV}$ photon of the ${ }^{60} \mathrm{Co}$ source, when tracking is used and single interaction events are removed, the full energy peak efficiency and peak-to-total values were $2.55(3) \%$ and $42.3(5) \%$, respectively.

The fast moving radioactive projectiles impinging on a secondary target can undergo nuclear or Coulomb excitation. The study of the nuclei produced in nuclear reactions, such as nucleon removal or secondary fragmentation, requires both $\mathrm{Z}$ and $\mathrm{A}$ identification of the reaction product. Typical specifications of the LYCCA array are $\Delta Z / Z=0.015$ and $\Delta A / A=0.012$ (FWHM) for $\sim_{36}^{80} \mathrm{Kr}$ fission-fragment beams [43]. A typical $\Delta \mathrm{E}$-E spectrum demonstrating the $Z$ identification capability of LYCCA is shown in Fig. 13 [51]. The LYCCA performance allows good element and isotope identification for lighter nuclei up to $A \sim 100$, while for heavy elements the isotope identification is not possible. In future, identification of heavy fragments will need magnetic rigidity measurement [53]. In contrast, since the probability of Coulomb excitation is much higher than that of nuclear excitation, in such measurements the requirements for ion identification is lower, allowing such experiments to be performed for any mass region. We note that Coulomb excitation at these energies is a single step process. Furthermore, the cross section is less dependent on the excitation energy when compared to experiments at the Coulomb barrier, allowing the population of several states $\left(2^{+}\right.$states in even-even nuclei).

Fragmentation facilities are characterized by beam cocktails normally consisting of several species. A typical identification plot where a whole range of nuclei are studied at the same time is shown in the left-hand side of Fig. 14 [51]. It was obtained in the in-flight fission of ${ }^{238} \mathrm{U}$. On the rightmost panel of the same figure an identification plot for an experiment devoted to the study of a single nucleus, ${ }^{206} \mathrm{Hg}$, produced in fragmentation is shown [52].

Often, the nucleus of interest is transmitted to the secondary target not only in its ground state but also in its longlived isomeric state(s). The nucleus can survive the flight-time of $\sim 300$ ns through the fragment separator if the isomeric half-life is comparable or longer than the flight-time. For isomers decaying by low-energy transitions in nuclei in bare ions (ions without electrons and charge $\mathrm{Q}=\mathrm{Ze}$ ) the conversion electron emission is blocked, therefore the effective half-life can be much longer than that of a neutral atom. The knowledge of the isomeric composition of the beam is often important. This is the case for example for Coulomb excitation experiments.

Examples of $\gamma$-ray spectra following Coulomb excitation at GSI are shown in Fig. 15. The nuclei of interest with an energy of $E / A=180 \mathrm{MeV}$ were excited in a single $500 \mathrm{mg} / \mathrm{cm}^{2}$ thick gold foil [54]. The relatively large background is apparent and typical for experiments at relativistic beam energies. The large beam energies make the GSI experiments 

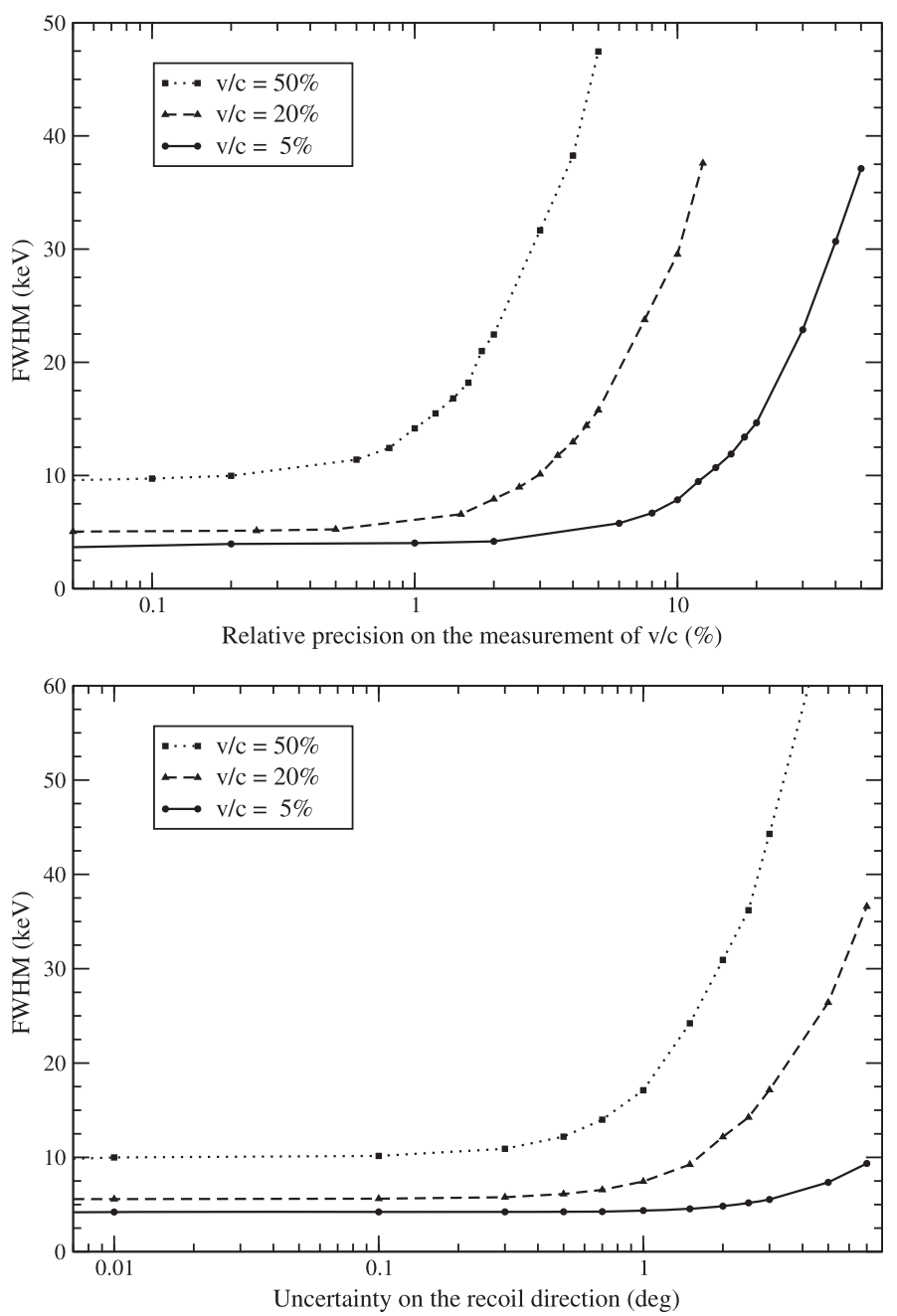

Fig. 12. Energy resolution (FWHM) of the AGATA array at $\mathrm{E}_{\gamma}=1 \mathrm{MeV}$ as function of the uncertainties on velocity $\beta$ and recoil direction. The indicated $v / c$ values of $5 \%, 20 \%, 50 \%$ correspond to energies of $\mathrm{E} / \mathrm{A} \approx 1,20$ and $145 \mathrm{MeV}$, respectively.

Source: Figure modified from [50].

particularly suited for lifetime measurements based on Doppler shift methods. A general technique uses the comparison of the energy of the Doppler corrected peak with the rest energy. The difference between them depends on the lifetime of the state. The method requires the knowledge of the rest energy and was used for neutron-rich Mo isotopes, as illustrated in Fig. 16. Another technique requiring higher statistics employs multiple targets to obtain lifetime information. A stack of three gold targets with thicknesses of $750,500,500 \mathrm{mg} / \mathrm{cm}^{2}$, separated by $1 \mathrm{~mm}$ gaps, were used. The size of the Doppler shift indicates the velocity of the moving nucleus, and it is determined by the position in the stack where it was emitted. The relative yields of the different Doppler shifted components depend on the lifetime. The technique is illustrated by the recorded $\gamma$ rays presented in Fig. 16.

The high energies of the beams at GSI allow the use of specific techniques and the study of phenomena not possible at lower energy accelerator facilities. Examples where the high energies are essential are the studies of low-lying dipole strength in radioactive nuclei following high energy (E/A $450 \mathrm{MeV})$ Coulomb excitation [55], M1 Coulomb excitation [56], and the importance of nucleonic degrees of freedom in high-energy reactions [57].

\subsection{AGATA at GANIL: features and performances}

The installation of AGATA at GANIL aims at covering a broad range of physics such as the study of shell evolution, understanding the microscopic origin of nuclear deformation and the interplay between single-particle and collective degrees of freedom, establishing shape coexistence and shape transitions and understanding the underlying mechanisms, probing the nature of pair correlations, measuring the degree of isospin-symmetry breaking. This ambitious program is 


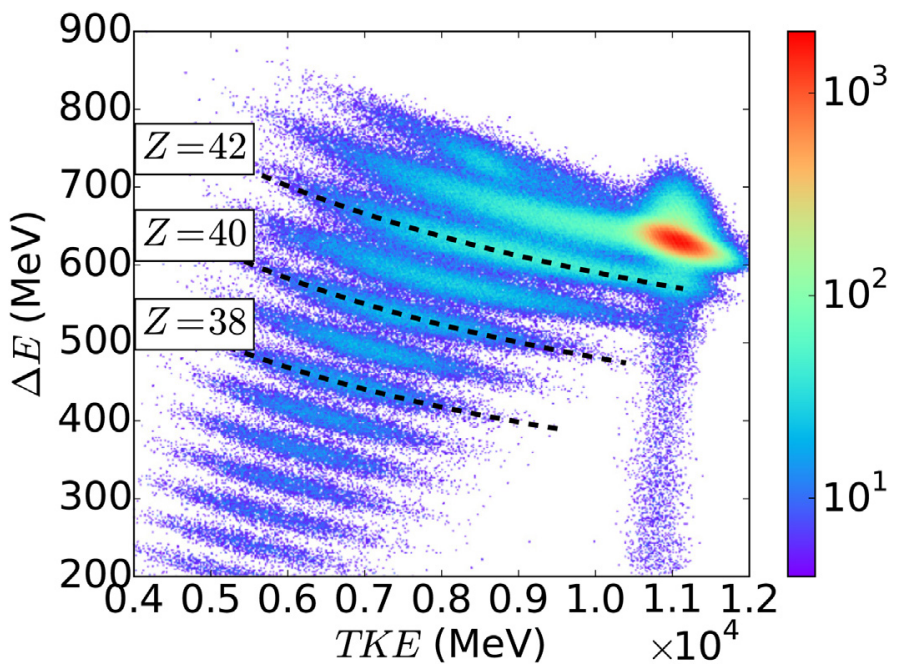

Fig. 13. $Z$ identification of secondary reaction products of ${ }^{109} \mathrm{Tc}$ ions on a beryllium target, as measured with LYCCA. In the vertical axis $\Delta \mathrm{E}$ is the energy loss and in the horizontal axis TKE is the kinetic energy of the beam.

Source: Figure taken from [51].
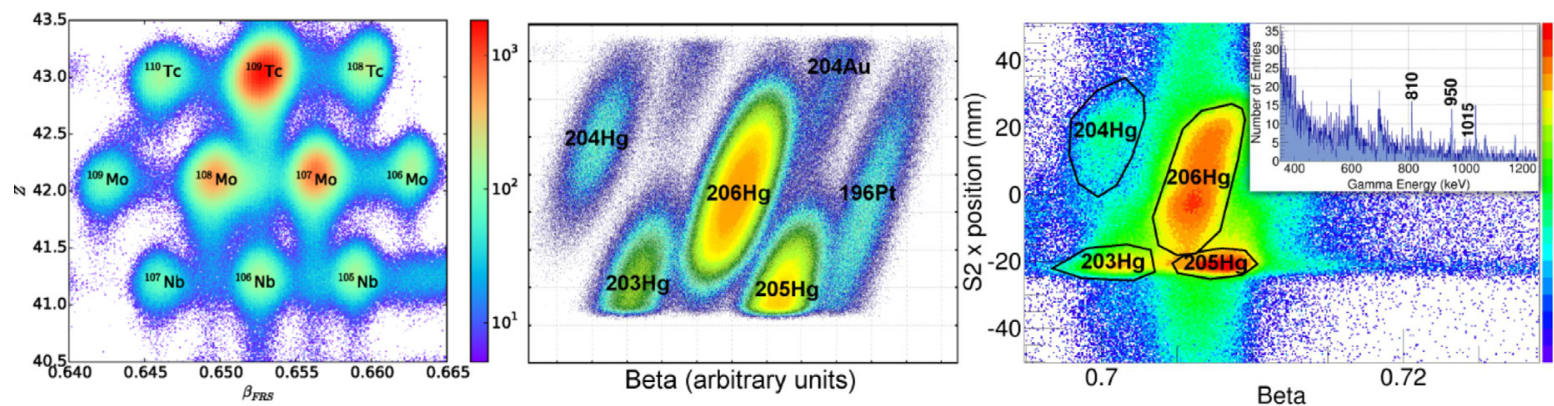

Fig. 14. (Leftmost panel): Identification plot for fission products centered on ${ }^{109} \mathrm{Tc}$. (Rightmost panel): Identification plot for products of fragmentation of a ${ }^{208} \mathrm{~Pb}$ beam, centered on ${ }^{206} \mathrm{Hg}$. The identification is confirmed by the comparison of measured and simulated data, the latter shown in the central panel. The delayed $\gamma$-ray spectrum associated with ${ }^{205} \mathrm{Hg}$, shown in the inset of the rightmost panel, proves the identification. Source: Figures taken from [51] and [52], respectively.
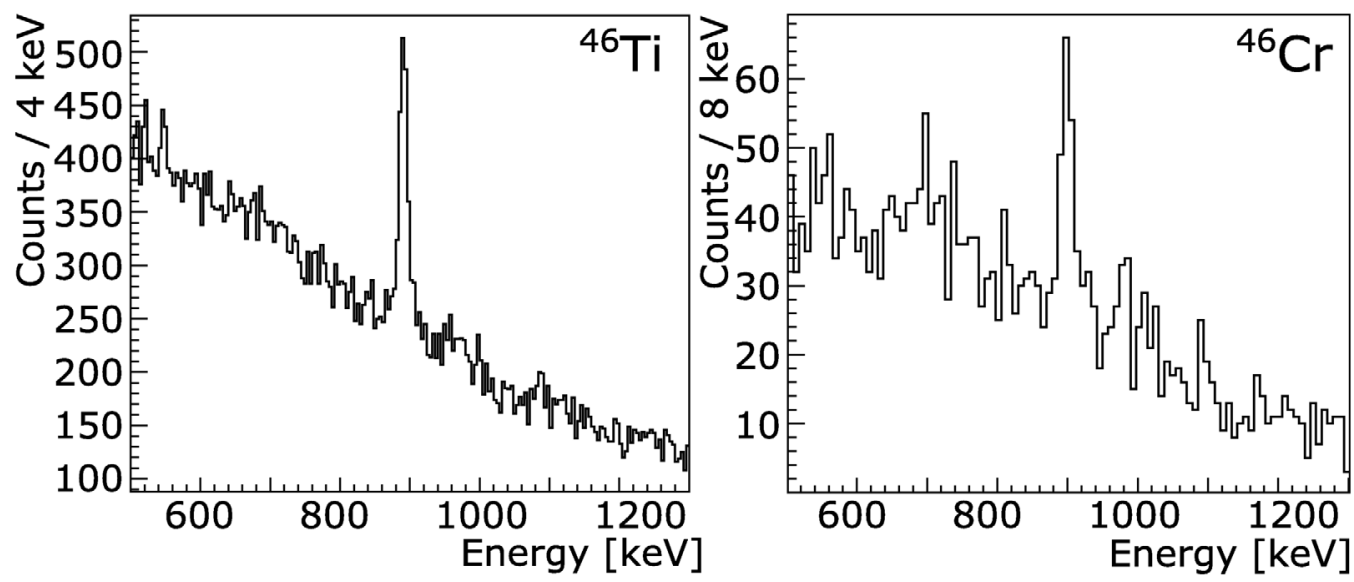

Fig. 15. $\gamma$-ray spectra following relativistic-energy Coulomb excitation of ${ }^{46} \mathrm{Ti}$ and ${ }^{46} \mathrm{Cr}$ nuclei. Source: Figure taken from [54]. 

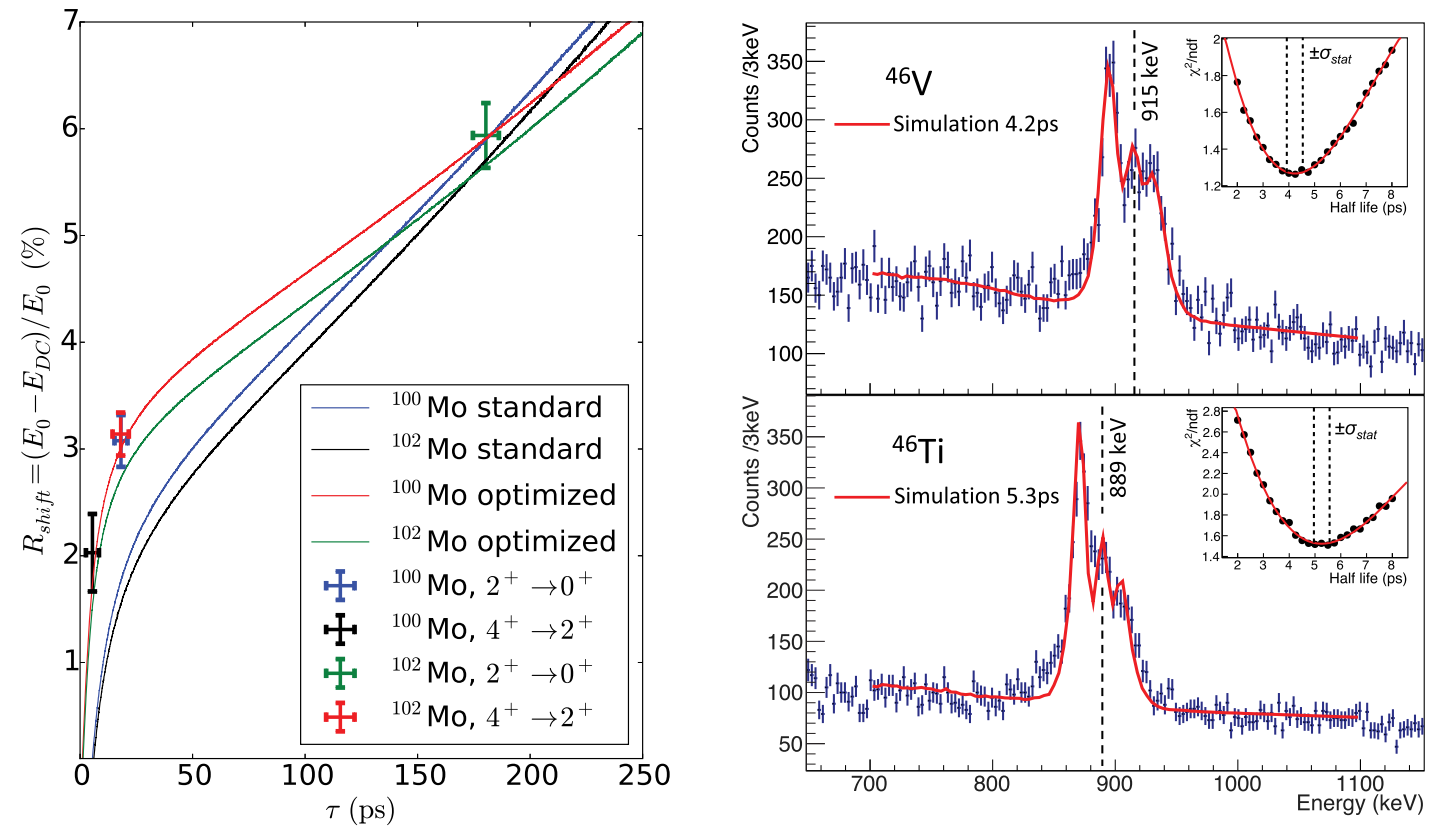

Fig. 16. Lifetime measurements using Doppler shift methods at GSI. Left panel): Mean lifetimes determined based on the difference between rest energies $\left(E_{0}\right)$ and Doppler corrected energies $\left(E_{D C}\right)$ of $\gamma$ rays. The lines are simulations for different sets of beam velocities and target thicknesses, with those labeled optimized being consistent with known lifetimes. The standard and optimized velocities correspond to $\beta=0.48$ and $\beta=0.44$, respectively. Figure taken from [51]. (Right panel): Line shape using three targets simultaneously. The measured and simulated spectra as well as the $\chi^{2}$ values as function of the assumed half-life are given for ${ }^{46} \mathrm{~V}$ and ${ }^{46} \mathrm{Ti}$. Figure taken from [54].
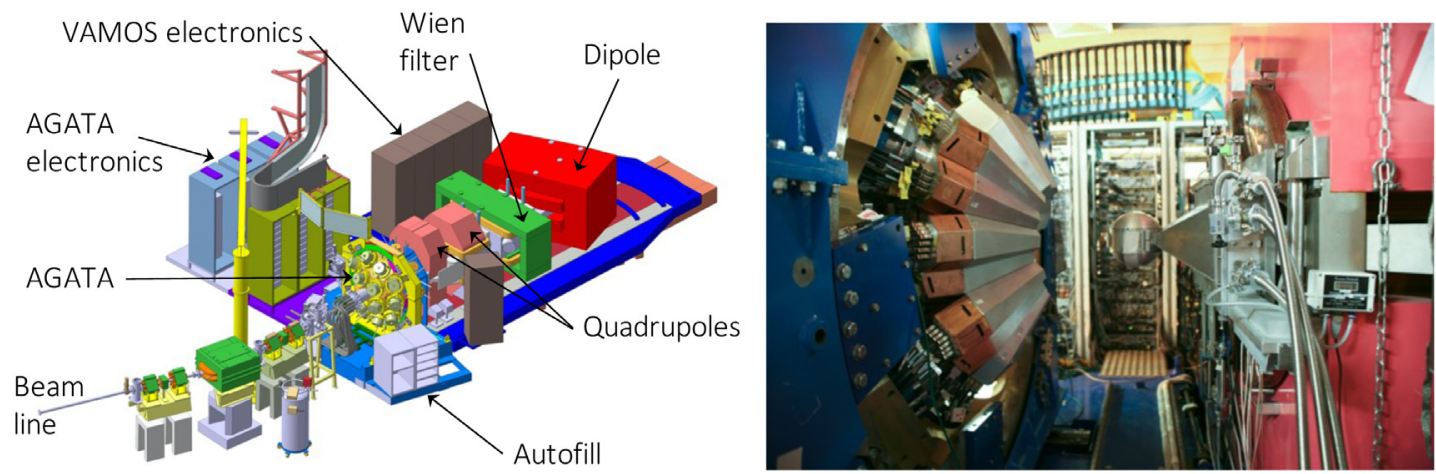

Fig. 17. Layout of the AGATA $1 \pi$ array coupled to the VAMOS++ spectrometer in the G1 experimental hall as installed at GANIL. Left panel: Figure adapted from [58]. Right panel: Photograph of AGATA (left hand-side), the reaction chamber and the VAMOS entrance cone (center) and VAMOS in red (right hand-side).

possible thanks to the use of a broad variety of stable beams and, in particular the intense $U$ beam as well as the exotic beams of SPIRAL1 and the coupling of AGATA to the mass spectrometer VAMOS++ in vacuum mode [30,31] and to other complementary detectors.

The full configuration of the AGATA array installed at GANIL consists of 15 ATCs assembled in two rings of 5 and 10 units [58]. AGATA is coupled to the VAMOS++ spectrometer [30,31] in the G1 experimental hall (Fig. 17). The ATC are placed at the most backward angles around the optical axis of the mass spectrometer, the nominal target to front end-cap distance being $23.1 \mathrm{~cm}$. The AGATA holding structure and the VAMOS++ spectrometer are placed on a common platform which can rotate perpendicularly to the beam axis at the target position up to $45 \mathrm{deg}$.

The AGATA honeycomb structure can be rotated by $\pm 90 \mathrm{deg}$ around the beam axis to ease the installation and removal of ATC in the array. In addition, the AGATA holding structure can be translated along the beam axis by $100 \mathrm{~mm}(400 \mathrm{~mm})$ towards (away from) the target.

At GANIL several experiments using a variety of stable and exotic beams were carried out. In particular, additional detectors were coupled to the AGATA array and they played a key role for the selective experiments carried out there. AGATA $1 \pi$ coupled to the VAMOS++ spectrometer in vacuum mode enabled the $\gamma$-ray spectroscopy up to medium spins 


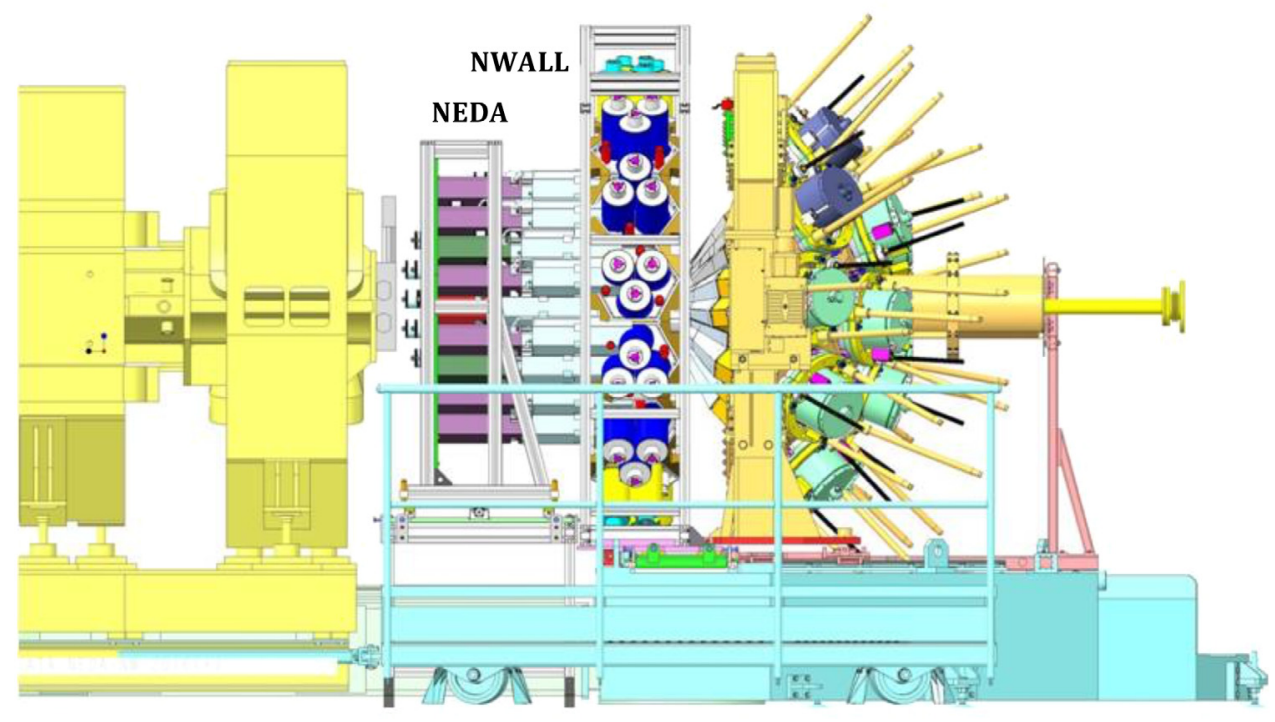

Fig. 18. Coupling of AGATA in compact configuration to NEDA and NWALL detectors, the VAMOS++ spectrometer being shifted by $1.1 \mathrm{~m}$ downstream. Source: Figure taken from [58].

in nuclei populated with fusion-fission or multi-nucleon transfer reactions. The detection systems of $\mathrm{LaBr}_{3}$ scintillators, FATIMA [59-61] and PARIS [62] were installed to improve the $\gamma$-ray detection efficiency for rare event studies. With the PARIS clusters $\gamma$-rays were measured up to high energy with a good timing feature, while the FATIMA array allowed measurements of lifetimes in pico-nano second range. In addition for lifetime measurements in the picosecond range the differential plunger to the target $[63,64]$ was used.

The unique feature of the AGATA configuration at GANIL was the neutron detection in coincidence with $\gamma$-rays which resulted to be instrumental, together with the detection of light charged particles, to study the unknown properties of nuclei with $\mathrm{N} \approx \mathrm{Z}$ in the medium mass region (see Section 4), particularly those at around ${ }^{100} \mathrm{Sn}$.

Indeed, shifting the VAMOS++ spectrometer by $1.1 \mathrm{~m}$, large detection systems such as the NEDA [65-67] and NWALL [68] neutron detectors were inserted (Fig. 18). This configuration allowed also to insert light charged-particle detection systems, namely DIAMANT [69] and GASPARD [70]. The latter allowed the spectroscopy of very neutron deficient nuclei around ${ }^{100} \mathrm{Sn}$ with AGATA shifted towards the target by about $10 \mathrm{~cm}$, the so-called compact configuration. Using SPIRAL1 beams and GASPARD [70], transfer reactions on exotic species were performed.

The response of the AGATA $1 \pi$ array has been simulated using the most advanced GEANT4 modeling of the detector and its environment. Both the nominal and the compact positions were considered. Efficiencies and peak-to-total $(\mathrm{P} / \mathrm{T})$ ratio were calculated using the calorimetric energies (sum of all energies deposited in the array) and after tracking using the MGT code with standard parameters [71]. The usual PSA data treatment performed on real data using the Adaptive Grid search Algorithm [24] was replaced in the simulation by packing interaction points spaced by less than $5 \mathrm{~mm}$. The evolution of the efficiency versus $\gamma$-ray energy is shown in Fig. 19.

The impact of the recoil velocity on the Doppler broadening has been investigated for velocities typical of recoils produced in multi-nucleon transfer, fusion-fission and fusion-evaporation reactions. Fig. 20 presents the evolution of the energy resolution normalized by the one at rest (called relative resolution) versus recoil velocity for both the nominal and compact positions. For the AGATA experiments using the NEDA, NWALL and DIAMANT detector systems, an important issue is the modification of the AGATA performances for $\gamma$-ray detection. Therefore, simulations were performed to estimate the impact on photo-peak efficiency and P/T of the DIAMANT CsI cells and the mutual Compton back-scattering of the NWALL and AGATA arrays (Fig. 21). The P/T values are calculated with a low-energy threshold of $20 \mathrm{keV}$ in the AGATA crystals. The reduced efficiency at low energy, due to $\gamma$-ray absorption in DIAMANT CsI cells, is clearly visible. The photopeak efficiency values at $122 \mathrm{keV}$ ( ${ }^{152} \mathrm{Eu}$ source) and at $1.3 \mathrm{MeV}\left({ }^{60} \mathrm{Co}\right.$ source) are reduced by $60 \%$ and $10 \%$, respectively. The $\mathrm{P} / \mathrm{T}$ ratio is larger after $\gamma$-ray tracking than in the calorimetric energy mode. It has also been demonstrated that the tracking efficiency is not affected by the presence of the DIAMANT array in the 200 to $1000 \mathrm{keV}$ range.

As a last point in this section, it is important to point out that also in the configuration of AGATA with VAMOS++ at GANIL it was possible to exploit well the $\gamma$-ray tracking capabilities of the array. An excellent example is the measurement of lifetimes in the few hundreds of femtosecond interval via the precise measurement of the line shape of the $\gamma$-ray transitions. This is discussed in Section 3 showing that indeed lifetimes were obtained thanks to the combination of two pieces of information, the tracking of $\gamma$-rays and the velocity distribution of the ions whose nuclei are emitting the $\gamma$-rays. In Fig. 22 selected results for the nucleus ${ }^{19} \mathrm{O}$ are shown from which one can appreciate the sensitivity of the lineshapes to lifetimes for two different transitions. 


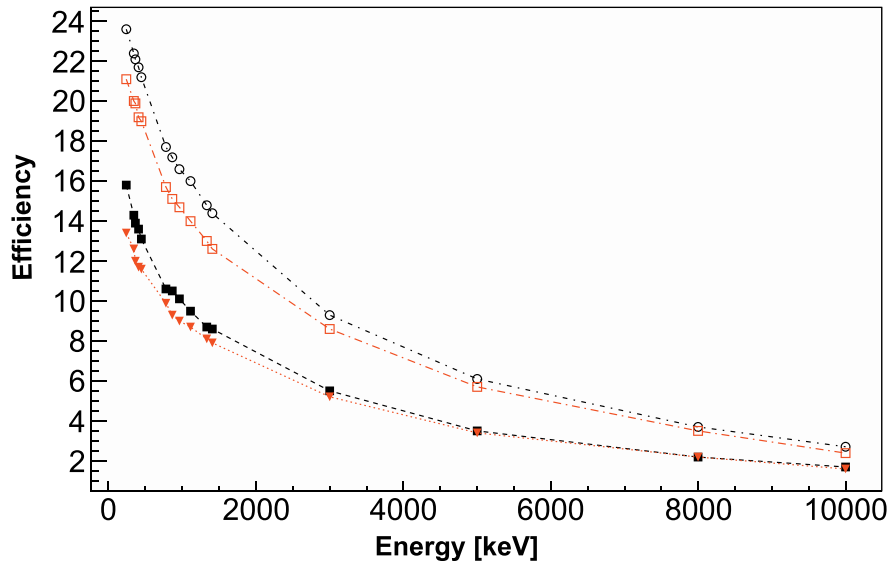

Fig. 19. Simulated photo-peak efficiency of the AGATA $1 \pi$ array as a function of the $\gamma$-ray energy after tracking. The full (open) symbols correspond to AGATA efficiency in nominal (compact) configuration. The tracked efficiency without and with target chamber are shown by the full black squares and full red triangles, respectively. The tracked efficiency with target chamber in compact configuration corresponds to the full black squares. The open red squares show the efficiency reduction due to the boost originating from in-flight emission with $\beta=0.1$. Source: Figure taken from [58].

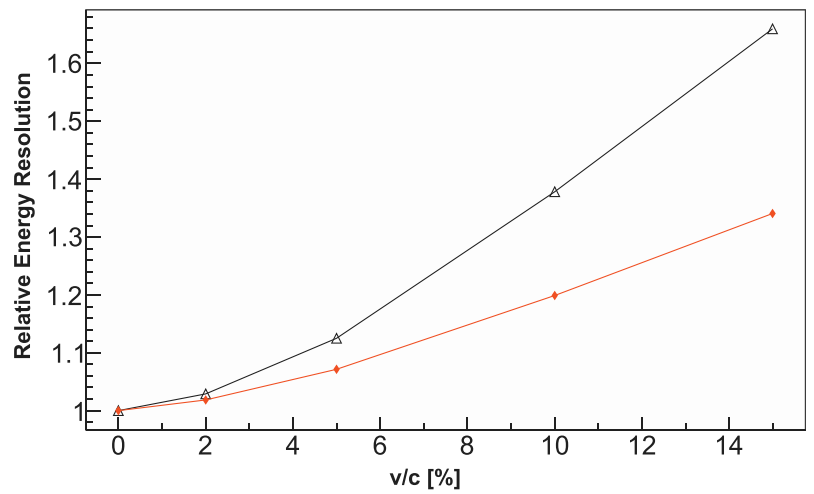

Fig. 20. Simulated relative energy resolution after tracking and Doppler correction plotted versus recoil velocity v/c for both the nominal (full red) and compact (open black) positions.

Source: Figure taken from [58].

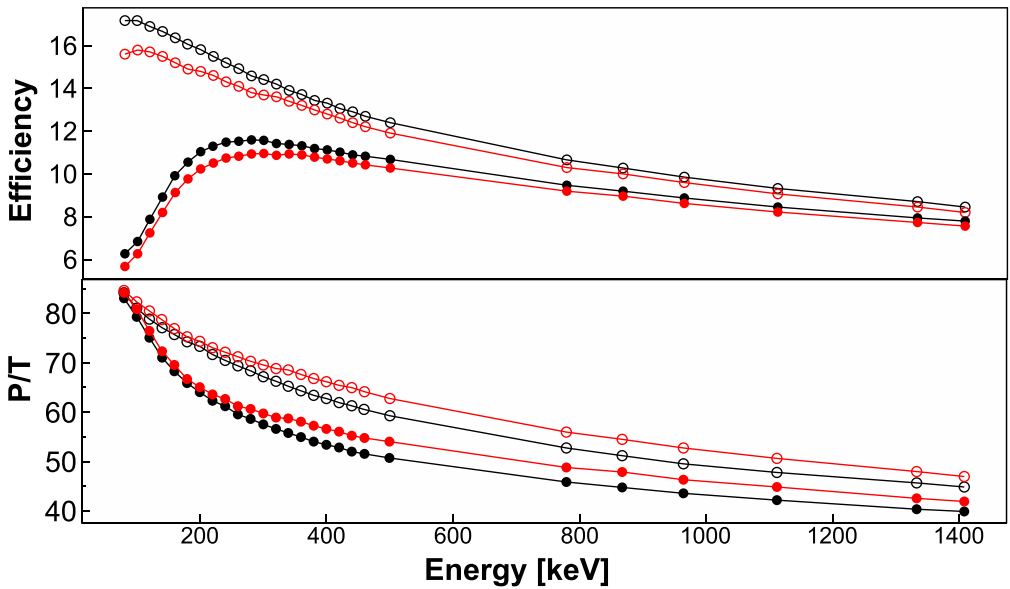

Fig. 21. Top panel: Simulated photopeak efficiency of AGATA $1 \pi$ in nominal position as a function of the gamma-ray energy. Open (full) symbols correspond to a configuration without (with) the NWALL and the DIAMANT detectors. Black and red symbols correspond to the results with the calorimetric energy and after tracking, respectively. Bottom panel: same for the $\mathrm{P} / \mathrm{T}$ ratio.

Source: Figure taken from [58]. 


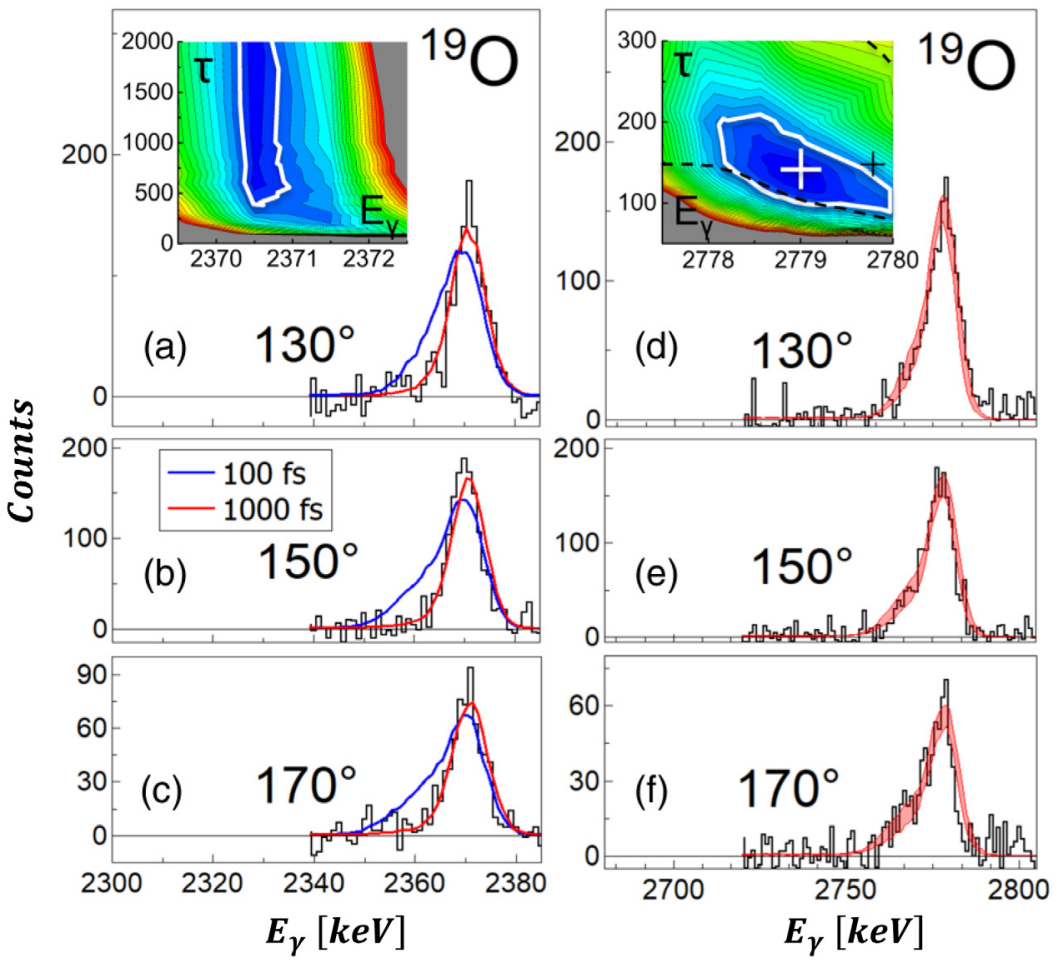

Fig. 22. Panels (a)-(c) and (d)-(f): $\gamma$-rays from the long-lived $(\tau>3.5 \mathrm{ps}) 9 / 2^{+}$state and from the $7 / 2^{+}$state in ${ }^{19} \mathrm{O}$, as measured by AGATA (precision $<4 \mathrm{~mm}$ ) in the angular ranges of $130^{\circ} \pm 10^{\circ}, 150^{\circ} \pm 10^{\circ}, 170^{\circ} \pm 10^{\circ}$. In (d)-(f) simulated spectra (shaded bands) are calculated on the basis of the global $\chi^{2}$ lifetime-energy surface (see the corresponding insets) with $1 \sigma$ uncertainty (white contour line) around the optimum $\gamma$-energy $\left(\mathrm{E}_{\gamma}\right)$ and lifetime $(\tau)$ values (white cross). The black cross and dashed lines indicate the $\chi^{2}$ minimum and $1 \sigma$ uncertainty for $\gamma$-detection angles defined by the AGATA front segment centers (precision $20 \mathrm{~mm}$ ). In (a)-(c), simulated spectra for $\tau=100$ and 1000 fs (blue and red lines, respectively) are shown to demonstrate the absence of broadening and tails for decays from long-lived states.

Source: Adapted from Ref. [72].

\section{Interactions properties from light nuclei}

One of the challenges in nuclear physics is to arrive to a unified description of all nuclei grounded on ab initio approaches using an Effective Field Theory (EFT) based on the symmetries and the relevant degrees of freedom of the QCD in the low-energy regime. Up to now ab initio predictions within the No-Core Shell Model (NCSM) or employing the Coupled-Cluster (CC) calculations were successful in describing ground and excited states of light nuclei even heavier than Oxygen [73]. The agreement of these results with experimental data demonstrated the potential of chiral EFT interactions, in particular of two- plus three-nucleon types. Concerning the effect of the three-nucleon $(3 \mathrm{~N})$ interaction it was found that by including $3 \mathrm{~N}$ forces (3NFs) in the calculations there is a significant improvement in the description of the measured binding energies and excitation spectra for neutron-rich oxygen isotopes [74]. In addition, the same approach reproduces well the excitation spectra of neutron-rich oxygen isotopes [75] and predicts that the nucleus ${ }^{24} \mathrm{O}$ is at the neutron drip line [76].

Another stringent test of the $a b$ initio predictions which could be rather sensitive to 3-body forces, is provided by the experimental information on the matrix element for E2 transitions in light nuclei. Such matrix element probes well the nuclear structure induced by this type of interaction. In particular, matrix elements are very sensitive to nuclear deformation, to the decoupling of proton and neutron degrees of freedom, and they are often affected by small components of the nuclear wave functions. Within the large-scale no-core shell model, using several nucleon-nucleon (NN) as well as NN plus three-nucleon (NNN) interactions, the electromagnetic $\mathrm{B}\left(\mathrm{E} 2 ; 2_{1}^{+} \rightarrow 0^{+}\right.$) transition rates, and the $2_{1}^{+}$quadrupole moments, as well as selected electromagnetic transitions among other states were calculated for neutron-rich carbon isotopes [77]. In spite of that the $\mathrm{B}(\mathrm{E} 2)$ values of the $2_{1}^{+}$transitions are found to be reproduced relatively well, a rather low sensitivity to NN or NNN nuclear force has been observed in the case of both neutron-rich carbon and oxygen isotopes. In fact, the difference among the predictions for these two type of interactions is only of the order of $5 \%-10 \%$. On the other hand, theory shows instead an enhanced sensitivity to other observables related to higher lying excitations. For the higher lying $2_{2}^{+}$state the predictions based on EFT for the energy and matrix elements $\mathrm{B}\left(\mathrm{M} 1 ; 2_{2}^{+} \rightarrow 2_{1}^{+}\right), \mathrm{B}\left(\mathrm{E} 2,2_{2}^{+} \rightarrow 2_{1}^{+}\right)$and $\mathrm{B}\left(\mathrm{E} 2 ; 2_{2}^{+} \rightarrow 0_{g . s}^{+}\right)$exhibit high sensitivity to the nuclear force used. In general, in the case of $\mathrm{C}$ and $\mathrm{O}$ neutron-rich isotopes the NN and NNN calculations differ by at least a factor $1.5-2[75,78]$. 

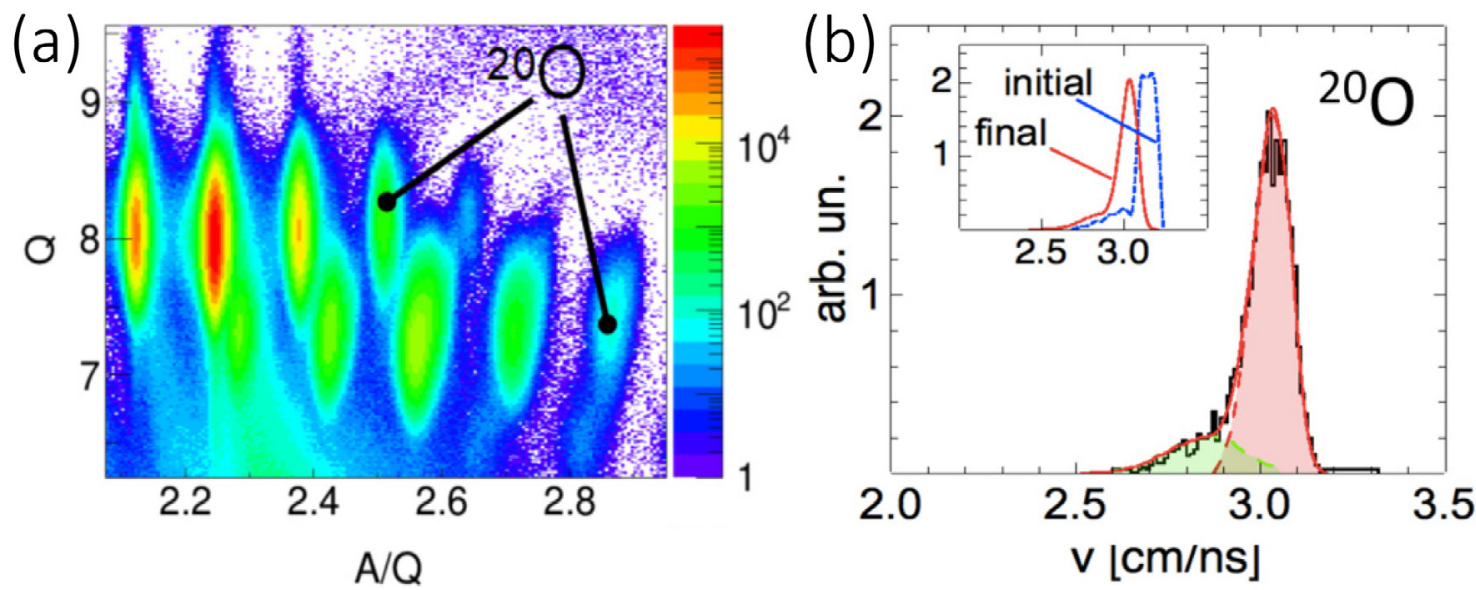

Fig. 23. Left panel: Identification plots, charge Q versus A/Q for $\mathrm{O}$ ions, as measured by VAMOS++. Right panel: Velocity distributions measured at the VAMOS++ focal plane (black histogram) in coincidence with the $2_{2}^{+} \rightarrow 2_{1}^{+}$transition of ${ }^{20} \mathrm{O}$ detected in AGATA. The solid red line is the simulated final velocity distribution of the quasi-elastic (red area) and deep-inelastic (green area) components. Inset: Reconstructed initial (dashed blue line) and final (solid red line) product velocities.

Source: Adapted from Ref. [72].

An experiment was performed with AGATA at the GANIL laboratory aiming to identify signals of NNN interactions in the structure of nuclei, in particular the light ones [72,79]. In fact, for these nuclei extensive theoretical work is ongoing since $a b$ initio demanding computations are accessible for them. Experimental information on the $2_{2}^{+}$states was not available and thus the focus of the experiment was on the measurements of these states to be characterized in terms of energy and matrix elements. The experiment used both the direct transfer and deep-inelastic processes induced by an ${ }^{18} \mathrm{O}$ beam at $126 \mathrm{MeV}$ on a ${ }^{181} \mathrm{Ta}$ target $\left(6.64 \mathrm{mg} / \mathrm{cm}^{2}\right)$. The beam energy at the center of the target was $\approx 116 \mathrm{MeV}$, i.e., $\approx 50 \%$ above the Coulomb barrier, and projectile like products had velocities of $\mathrm{v} / \mathrm{c} \approx 10 \%$. The AGATA array was coupled to two large volume (3.5 x 8 inches) LaBr3 detectors plus two clusters of the PARIS setup [62], which produced an excellent time reference for the reaction.

Reaction products were detected in the VAMOS++ spectrometer [30,80], placed at the reaction grazing angle of 45deg (opening angle $\pm 6 \mathrm{deg}$ ) with respect to the beam direction. AGATA covered the angular range $115 \mathrm{deg}-175 \mathrm{deg}$ whereas the scintillators were placed at 90deg relative to the VAMOS++ spectrometer. The VAMOS++ setting allowed detection of other products with charge $Z$ in the interval $Z=5-9$ and mass $A$ interval $A=11-21$. In the left panel of Fig. 23 the identification plot including the $\mathrm{O}$ ions is shown together with the velocity distribution of the ${ }^{20} \mathrm{O}$ ions when a condition on the excitation of the $2_{2}^{+}$is imposed (see the right panel). One can note that the velocity distribution is characterized by the presence of a pronounced peak, corresponding to the direct population in a quasi-elastic process, and a tail, extending towards lower velocities, which is associated with higher kinetic-energy losses. A portion of the $\gamma$-ray spectrum for the nucleus ${ }^{20} \mathrm{O}$ is shown in Fig. 24 obtained after applying the Doppler correction using the velocity vector of the emitting nucleus measured in VAMOS++. All visible $\gamma$ rays correspond to known transitions (see level scheme in Fig. 24 right). By examining the shape of the peaks one realizes that some peaks are narrow and their energies agree, within uncertainty, with earlier studies. This is the case of transitions from relatively long-lived levels ( $\tau>1 \mathrm{ps}$ ), emitted in flight outside the target as, for example, the 1674-keV line. In contrast, other lines, depopulating states with lifetimes shorter than 1 ps, exhibit rather large widths and tails. These features indicate that the corresponding $\gamma$ rays were partly emitted during the stopping process of the reaction product inside the target and thus at velocities larger than the one measured in the spectrometer. These Doppler-broadened line shapes were analyzed to determine the lifetime of the states of interest.

In order to extract the lifetime information from the measured line shapes, a simulation of the Doppler-shifted $\gamma-$ transition line shapes was performed. The first step was to reconstruct interactively the initial velocity distribution of the $\gamma$ emitting nucleus inside the target, associated with the population of a given state (see the inset in the right panel of Fig. 24). The quantities used for this simulation were: (i) the measured distribution of the angle of the velocity; (ii) the reaction kinematics calculated for the selected states assuming population from both a direct and more dissipative processes; (iii) a random probability of the reaction occurrence over the target thickness, and (iv) the slowing-down and straggling processes inside the target. The simulated results for the emitter were then employed to simulate the Dopplershifted energy measured in AGATA, the lifetime and $\gamma$-transition energy was treated as a free parameter. The angular uncertainty for the $\gamma$ direction was $\approx 1.5 \mathrm{deg}$ (as a combined from the uncertainties of 1deg for AGATA and 1deg for VAMOS++). After that the $\chi^{2}$ was minimized in lifetime-energy coordinates.

The obtained result for the $2_{2}^{+}$state in ${ }^{20} \mathrm{O}$ is $\mathrm{E}_{\gamma}=2394.6_{-1.0}^{+1.0} \mathrm{keV}$ and $\tau=150_{-30}^{+80}$ fs. The found $\gamma$-ray energy agrees within the uncertainties with the most precise value reported in literature, i.e., $\mathrm{E}_{\gamma}=2396(1) \mathrm{keV}$. Calculations were performed for this nucleus by employing chiral NN interactions based on Ref. [81], and 3N interactions fitted on top, 
$x 10^{3}$

(a)

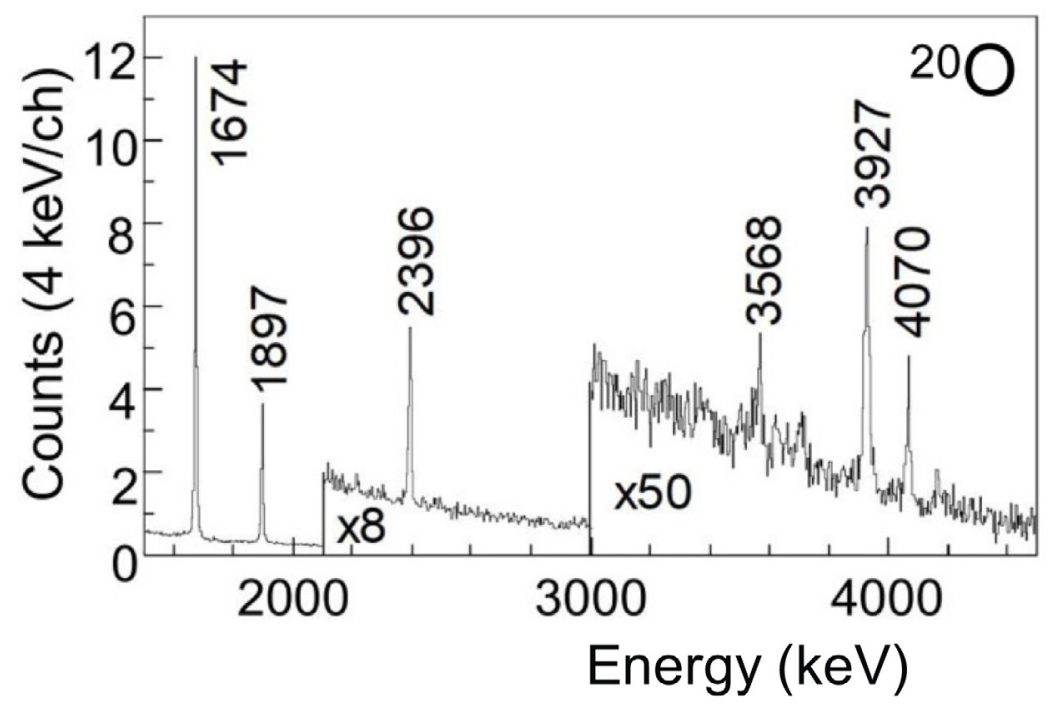

(b)

$\left(3^{-}\right)$

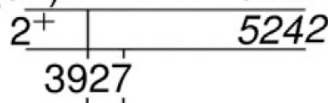

। 1

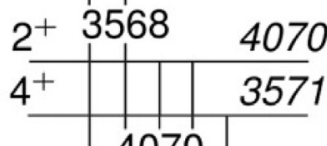

4070

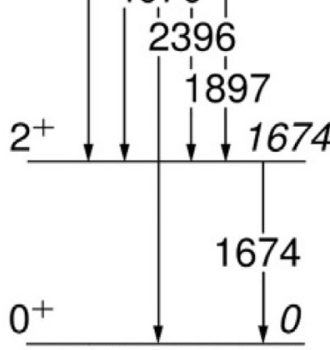

Fig. 24. Left panel: $\gamma$-ray spectrum (a) of ${ }^{20} \mathrm{O}$ as measured by AGATA. Right panel: Corresponding level scheme (b)of ${ }^{20} \mathrm{O}$. Source: Adapted from Ref. [72].
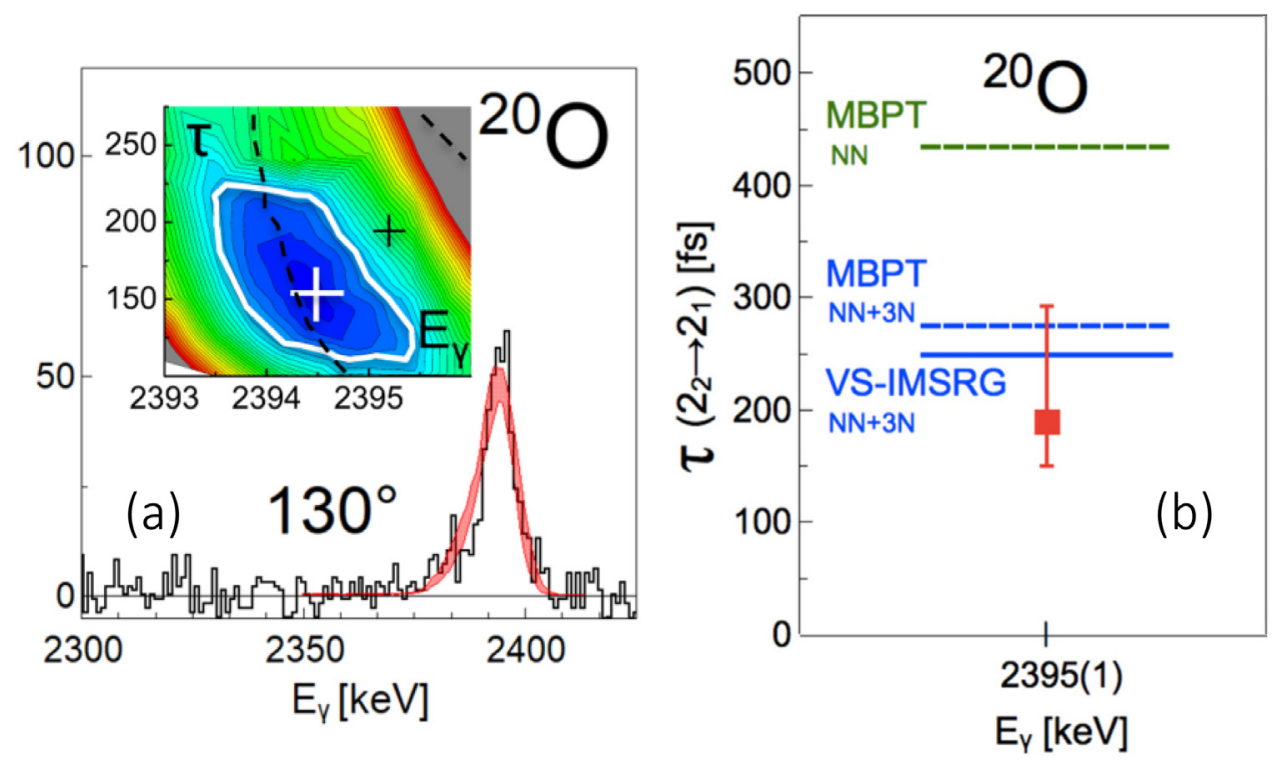

Fig. 25. Left panel: Spectrum for the $2396-\mathrm{keV} \gamma$ transition from the long-lived $\left(\tau=3.5 \mathrm{ps}\right.$ ) $2_{2}^{+}$state in ${ }^{20} \mathrm{O}$ as measured by AGATA (precision $<$ $4 \mathrm{~mm}$ ) at $130^{\circ}$. The simulated spectrum is shown with a red line which is associated to the lifetime corresponding to the value of the minimum of the global $\chi^{2}$ lifetime-energy surface (see the inset). Right panel: Partial lifetime for $2_{2}^{+} \rightarrow 2_{1}^{+}$decays of ${ }^{20} \mathrm{O}$. The experimental value (symbol) is compared to MBPT predictions (dashed lines, with and without $3 \mathrm{~N}$ interactions), and to an ab initio VS-IMSRG prediction (solid line). The MBPT results use neutron effective charge $e_{n}=0.4$ and g-factors $g_{s}=-3.55$ and $g_{l}=-0.09$. The acronyms MBPT and VS-IMSRG are explained in the text.

Source: Adapted from Ref. [72].

considering only few-body systems up to ${ }^{4} \mathrm{He}$. In particular, the many-body perturbation theory (MBPT) valence shell interactions from Ref. [75], were employed with an ${ }^{16} \mathrm{O}$ core. $\mathrm{NN}$ and normal-ordered $3 \mathrm{~N}$ interactions were included up to third order, neglecting residual $3 \mathrm{~N}$ interactions. Effective operators were used to calculate EM transitions [82,83]. The MBPT results reproduce well the $2_{1}^{+} \rightarrow 0^{+}$lifetime in ${ }^{20} \mathrm{O}(\tau=11.7 \mathrm{ps}$ versus the experimental $\tau=10.5(4)$ ps [84]), and this agreement does not depend significantly on the inclusion of $3 \mathrm{~N}$ interactions. In turn, as shown in the right panel of Fig. 25, the calculated partial lifetime of the $2_{2}^{+} \rightarrow 2_{1}^{+}$decay, $\tau=275$ fs (the dashed blue line), agrees well with the present 
Table 2

Experimental data deduced from the lifetime $\tau$ of the state and predictions for ${ }^{21} \mathrm{O}$ from [91] are in the first row. The calculated branching ratio (BR) uses the predicted $\mathrm{B}(\mathrm{E} 2)$ and $\mathrm{B}(\mathrm{M} 1)$ values and the experimental transition energies. The calculated $\tau_{1 / 2+}$ uses the experimental transition energy. The errors for the IM-NCSM are due to the uncertainties of the many-body method.

\begin{tabular}{|c|c|c|c|c|c|c|c|}
\hline & $\begin{array}{l}\tau(p s) \\
1 / 2^{+}\end{array}$ & $\begin{array}{l}\mathrm{BR}(\%) \\
3 / 2^{+} \rightarrow 1 / 2^{+}\end{array}$ & $\begin{array}{l}\mathrm{B}(\mathrm{E} 2)\left(\mathrm{e}^{2} \mathrm{fm}^{4}\right) \\
1 / 2^{+} \rightarrow 5 / 2^{+} \mathrm{gs}\end{array}$ & $\begin{array}{l}\mathrm{B}(\mathrm{E} 2)\left(\mathrm{e}^{2} \mathrm{fm}^{4}\right) \\
3 / 2^{+} \rightarrow 1 / 2^{+}\end{array}$ & $\begin{array}{l}\mathrm{B}(\mathrm{E} 2)\left(\mathrm{e}^{2} \mathrm{fm}^{4}\right) \\
3 / 2^{+} \rightarrow 5 / 2^{+} \mathrm{gs}\end{array}$ & $\begin{array}{l}\mathrm{B}(\mathrm{M} 1)\left(10^{-3} \mu_{N}\right) \\
3 / 2^{+} \rightarrow 1 / 2^{+}\end{array}$ & $\begin{array}{l}\mathrm{B}(\mathrm{M} 1)\left(10^{-3} \mu_{N}\right) \\
3 / 2^{+} \rightarrow 5 / 2^{+}\end{array}$ \\
\hline Experiment & $420_{-32-12}^{+34+35}$ & $11.7 \pm 1.2$ & $0.71_{-0.06-0.06}^{+0.07+0.02}$ & - & - & - & - \\
\hline USDB & $176^{-32-12}$ & 20.3 & $1.69^{-0.00-0.00}$ & 2.06 & 3.54 & 5.6 & 0.6 \\
\hline VS-IMSRG & 704 & 12.7 & 0.42 & 0.61 & 0.55 & 5.3 & 2.6 \\
\hline $\mathrm{N}^{2} \mathrm{LO}_{S A T}$ & $444_{-81}^{+128}$ & $4.6_{-2.6}^{+5.5}$ & $0.67 \pm 0.15$ & $0.63 \pm 0.05$ & $0.79 \pm 0.06$ & $3.0 \pm 1.2$ & $4.6 \pm 1.9$ \\
\hline $\mathrm{N}^{3} \mathrm{LO}_{E M}+\mathrm{N}^{2} \mathrm{LO}_{L, 400}$ & $804 \pm 22$ & $12.5_{-2.5}^{+3.2}$ & $0.37 \pm 0.01$ & $0.47 \pm 0.05$ & $0.55 \pm 0.02$ & $9.8 \pm 1.0$ & $5.1 \pm 0.8$ \\
\hline $\mathrm{N}^{3} \mathrm{LO}_{E M}+\mathrm{N}^{2} \mathrm{LO}_{N L, 500}$ & $488 \pm 25$ & $14.1_{-1.9}^{+2.4}$ & $0.61 \pm 0.03$ & $0.74 \pm 0.08$ & $0.77 \pm 0.04$ & $8.4 \pm 0.4$ & $3.7 \pm 0.5$ \\
\hline $\mathrm{N}^{4} \mathrm{LO}_{E M N}+\mathrm{N}^{2} \mathrm{LO}_{N L, 500}$ & $513_{-69}^{+94}$ & $15.5 \pm 1.3$ & $0.58 \pm 0.09$ & $0.70 \pm 0.07$ & $0.77 \pm 0.06$ & $8.2 \pm 0.5$ & $3.2 \pm 0.1$ \\
\hline
\end{tabular}

measurement only when $3 \mathrm{~N}$ interactions are considered. The agreement with the calculations appears to be driven by the dominant $\left(d_{5 / 2}\right)^{3}\left(s_{1 / 2}\right)^{1}$ configuration, which is mostly missing in the NN calculation.

$\mathrm{Ab}$ initio calculations within the VS IMSRG model (see e.g. Refs. [75,85-87]) based on the $\mathrm{NN}+3 \mathrm{~N}$ interaction labeled EM1.8/2.0 in Ref. [88] are also available. In these calculations the treatment of $3 \mathrm{~N}$ force improves that of the MBPT by including interactions between valence nucleons [87]. The computed transition energies (1629 and $2422 \mathrm{keV}$, for the $2_{1}^{+}$ $\rightarrow 0^{+}$and $2_{2}^{+} \rightarrow 2_{1}^{+}$, respectively) are found to be in very good agreement with experiment. This is at variance with the MBPT results, which overestimate the experimental energies by about $400 \mathrm{keV}$. The partial lifetime in ${ }^{20} \mathrm{O}, \tau=249 \mathrm{fs}$ (the solid line), also agrees very well with the experimental value. The $\mathrm{B}(\mathrm{M} 1)=0.0166 \mu_{N}^{2}$ dominates over $\mathrm{B}(\mathrm{E} 2)=0.0684$ $\mathrm{e}^{2} \mathrm{fm}^{4}$. The good agreement with the experimental lifetime also suggests a small impact of meson-exchange currents, not included in this calculation.

Other new data were obtained also for ${ }^{16} \mathrm{C}$ and compared with calculations [72]. In that case the statistics was more limited but it was possible to extract with $78 \%$ probability an energy $\mathrm{E}_{\gamma}>2216 \mathrm{keV}$ for the $2_{2}^{+} \rightarrow 2_{1}^{+}$transition and a lifetime of $\tau<180 \mathrm{fs}$.

These results are important building blocks for the construction of a unifying approach and show at the same time the need of several data in this mass region in order to benchmark interactions and constantly evolving computational approaches. Within the efforts made in this connection, it is interesting to recall here the recent result on lifetimes measurements for ${ }^{21} \mathrm{O}$. The $\mathrm{B}(\mathrm{E} 2)$ value for the transition from the first excited state to the ground state was measured for the odd-even oxygen isotopes ${ }^{17,19} \mathrm{O}[89,90]$. The measurements are found to be characterized by a sharp decrease as compared with the neighboring even-even isotopes. Thus, there is a need to verify if this behavior is also present in the more neutron-rich ${ }^{21} \mathrm{O}$. It is interesting in particular to see whether or not theory can describe well this behavior in a wide interval of isotopes. The measurement of lifetimes for the ${ }^{21} \mathrm{O}$ nucleus was performed at the laboratory NSCL (Michigan, USA) using the array GRETINA and the Recoil Distance Method (RDM) allowing the detection of $\gamma$ rays emitted before or after a degrader and thus with different Doppler shifts.

The obtained result is reported in Table 2 together with a set of different predictions based on various types of calculations [91]. One of these calculations uses a standard shell model with the commonly used residual interaction for the sd orbitals, another uses the VS-IMSRG approach based on 3N interaction, and instead the additional four calculations in the table all use ab initio valence-space Hamiltonians with $\mathrm{NN}+3 \mathrm{~N}$ forces derived from chiral EFT. The results corresponding to four types of forces are given in the table. The comparison of the datum for ${ }^{21} \mathrm{O}$ with this ensemble of predictions allows to assess the sensitivity to the input Hamiltonian. From the comparison between experiment and theory presented in [91] it was found that interactions derived with nonlocal $3 \mathrm{~N}$ regulators give a better account of the measured transition rates. In addition, as discussed in [91] this datum allows to infer the value of the shell-model effective charges since there the mixing with $2 \mathrm{p}-2 \mathrm{~h}$ excitations should be negligible.

Altogether these experiments with AGATA and GRETINA have well exploited the good determination of the spatial position that are allowed by these arrays to perform lifetime measurement in the interval $100-500 \mathrm{fs}$. It was possible to measure lifetimes of excited nuclear states populated with deep-inelastic collisions and fragmentation reactions [91]. Electric and magnetic $\gamma$-decays are found to provide a more demanding complementary test of theoretical ab initio approaches and to be selective in the role of $3 \mathrm{~N}$ forces. These experiments are expected to pave the way to further measurements in this mass region which is ideal to benchmark calculations of nuclear structure based on first principles.

\section{Single-particle excitations and new shells}

Since almost four decades, a vigorous effort is undertaken to study the structure of nuclei far away from the valley of stability, also called exotic nuclei. The known magic numbers of nucleons Z,N = 8, 20, 28, 50, 82 and 126 ensure an increased binding energy to stable nuclei, in particular to doubly-magic nuclei. However, several discoveries established that, neutron-rich nuclei with $\mathrm{N}=8,20$ and 28 could present structures of deformed nuclei [92-95], demonstrating an erosion of the binding energy for these numbers and the appearance of new, local magic numbers such as $\mathrm{N}=16$ [96] and $\mathrm{N}=32$, 34 [97-99]. Therefore, there has been a great effort both experimentally and theoretically all over the world to 

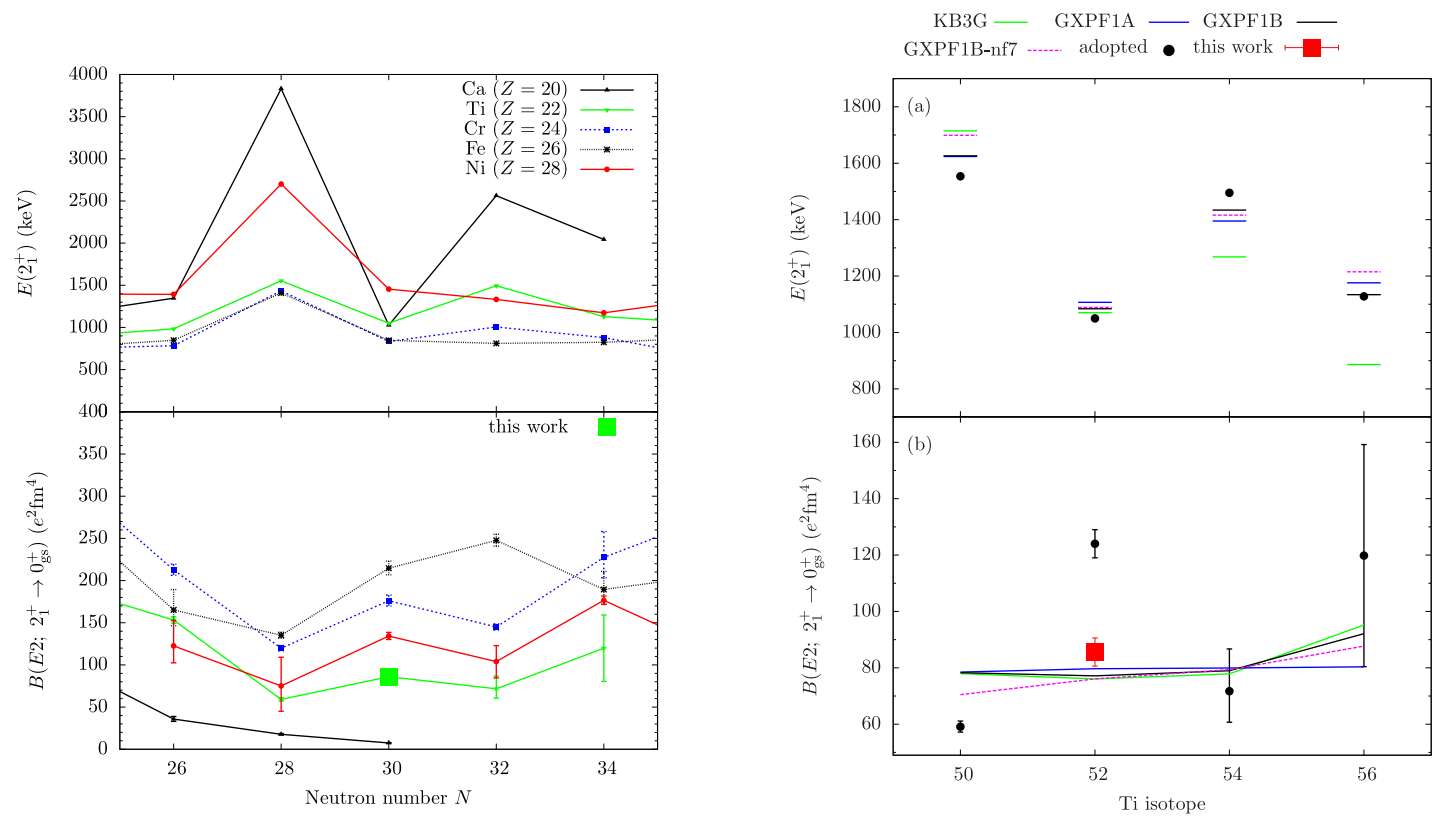

Fig. 26. Left panel: Systematics for $26<\mathrm{N}<34$ of the $2_{1}^{+}$state excitation energy and $\mathrm{B}\left(\mathrm{E} 2 ; 2_{1}^{+} \rightarrow 0_{g s}^{+}\right)$transition strengths from Ca to Ni. Right panel: Comparison of experimental $2_{1}^{+}$excitation energies (a) and $\mathrm{B}\left(\mathrm{E} 2 ; 2_{1}^{+} \rightarrow 0_{g s}^{+}\right)$values (b) in Ti isotopes with results of several shell-model calculations using different interactions.

Source: Adapted from [106].

study the nuclear structure evolution around both the magic numbers and the new emerging local magic numbers [100]. In the present section, spectroscopic studies performed with AGATA are presented around the subshell at $\mathrm{N}=32$ and $\mathrm{N}$ $=50, \mathrm{Z}=50$ shell closures. It is worth mentioning the complementary studies performed with GRETINA in the vicinity of other shells or subshells, namely $\mathrm{N}=20$ and 28 with some overlap for the subshells $\mathrm{N}=32$ and $\mathrm{N}=40$. Whereas in the present section, the nuclei studied with AGATA were populated using multi-nucleon transfer and fusion-fission reactions, the one- or two-nucleon knockout reactions were mainly used for these studies with GRETINA even though pickup and multi-nucleon transfer reactions, inelastic proton scattering and multistep Coulomb excitation were also used.

\section{1. $N=32$ sub-shell closure}

Specific interactions between nucleons in shell-model orbitals are often found to be at play and better isolated in nuclei far from stability. Notably a strong impact of the proton-neutron tensor force between the $j=1+1 / 2$ and $j=1-1 / 2$, spin flipped orbitals on single-particle energy shifts when filling or emptying one of these orbital was predicted in [101]. Such orbital migration was experimentally observed in the ${ }^{71-77} \mathrm{Cu}$ isotopes [102-104]. Indeed, in this isotopic series the first $5 / 2^{-}$state with a $\pi \mathrm{f}_{5 / 2}$ single-particle configuration drops from $1214 \mathrm{keV}$ in ${ }^{69} \mathrm{Cu}$ (empty $\nu g_{9 / 2}$ orbital) down to the ground state in ${ }^{75} \mathrm{Cu}$ ( 6 neutrons in $\nu g_{9 / 2}$ orbital) due to the strong $\pi f_{5 / 2}-v g_{9 / 2}$ interaction leading to the inversion of the $\pi p_{3 / 2}$ and $\pi f_{5 / 2}$ orbitals and to the change of ground-state spin from $3 / 2^{-}$in ${ }^{73} \mathrm{Cu}$ and lighter odd-even Cu isotopes to $5 / 2^{-}$in ${ }^{75} \mathrm{Cu}$ and heavier odd-even $\mathrm{Cu}$ isotopes. The larger the orbital momenta involved and the larger the orbital occupation, the stronger the tensor force.

Later, the same mechanism is invoked to explain shell-gap evolution versus proton or neutron number. By extension, closing or opening of new energy gaps were deduced as illustrated in the Ca isotopes with the appearance of new weak sub-shell gaps at $\mathrm{N}=32$ and 34 [98]. In the latter case, the interaction between the $\pi f_{7 / 2}$ and $\nu f_{5 / 2}$ orbitals lowers the $\nu f_{5 / 2}$ orbital in ${ }^{60} \mathrm{Fe}$, the latter being placed below the $\nu \mathrm{p}_{3 / 2}$ orbital. By removing protons from $\pi \mathrm{f}_{7 / 2}$, the mutual attraction is progressively released leading to the migration of the $\nu \mathrm{f}_{5 / 2}$ orbital across the $\nu \mathrm{p}_{3 / 2}$ orbital and opening the $\mathrm{N}=32$ gap. By emptying completely the $\pi \mathrm{f}_{7 / 2}$ orbital $\left({ }^{54} \mathrm{Ca}\right)$ a second sub-shell $\mathrm{N}=34$ gap is created. Consequently, a large $2^{+}$excitation energy is observed in ${ }^{52} \mathrm{Ca}$ as well as in the ${ }^{54} \mathrm{Ti}$ and ${ }^{56} \mathrm{Cr} \mathrm{N}=32$ isotones with a reduced strength but no $\mathrm{N}=32$ sub-shell effect is observed anymore in ${ }^{58} \mathrm{Fe}$ and ${ }^{60} \mathrm{Ni}$ (see Fig. 26 left panel). Simultaneously, small B(E2) values are deduced as expected at shell or sub-shell closure. This induces a staggering between $\mathrm{N}=28$ and $\mathrm{N}=32$ that is abnormally large in ${ }^{52} \mathrm{Ti}$ [105] and which does not agree with shell-model predictions. In addition, in ${ }^{50,54} \mathrm{Ti}$ the measured $\mathrm{B}(\mathrm{E} 2)$ values versus spin are relatively large for the lowest $2_{1}^{+} \rightarrow 0_{g s}^{+}$and $4_{1}^{+} \rightarrow 2_{1}^{+}$transitions and low for $6_{1}^{+} \rightarrow 4_{1}^{+}$whereas in ${ }^{52} \mathrm{Ti}$ the measured $\mathrm{B}(\mathrm{E} 2)$ value of the $4_{1}^{+} \rightarrow 2_{1}^{+}$transition is the lowest. 
To clarify the anomalies observed in Ti isotopes, the multi-nucleon transfer reaction ${ }^{238} \mathrm{U}+{ }^{50} \mathrm{Ti}$ has been chosen to feed relatively high-spin states in neutron-rich Ti isotopes combined with the Recoil-Distance Doppler-Shift method. Gammarays were detected in AGATA coupled to VAMOS++ placed at the grazing angles at $45 \mathrm{deg}$. The plunger device was equipped with a natural $\mathrm{Mg}$ degrader foil enabling data taking at 6 different target-to-degrader distances ranging from $\approx 70$ to $\approx$ $1000 \mu \mathrm{m}$ and giving access to lifetimes comprised between a few ps and about $400 \mathrm{ps}$. B(E2) values for $2^{+}, 4^{+}$and $6^{+}$ yrast states differ by about a factor 2 from the previous work. In particular, the revised $\mathrm{B}\left(\mathrm{E} 2 ; 2_{1}^{+} \rightarrow 0_{g s}^{+}\right)$value in ${ }^{52} \mathrm{Ti}$ reduces the staggering amplitude in the ${ }^{50-54} \mathrm{Ti}$ series with a rather flat behavior around $\mathrm{N}=30$ [106]. The new results compare better to shell-model calculations using several interactions. For ${ }^{50} \mathrm{Ti}$ it was found that it is necessary to lower the single-particle energy of the $\nu f_{7 / 2}$ orbital by $1 \mathrm{MeV}$ to describe well the data. However, both the experimental and the theoretical results confirm a local subshell closure at $\mathrm{N}=32$ in ${ }^{54} \mathrm{Ti}$, which is weaker to the one established at $\mathrm{N}=$ 28.

The ${ }^{54} \mathrm{Ti}$ isotope has also been studied with GRETINA using an inverse-kinematic proton inelastic-scattering experiment and a one-neutron knockout reaction from ${ }^{55} \mathrm{Ti}$ [107]. The former reaction favors the population of low-lying collective octupole states which is not the case of the multi-nucleon transfer reaction also used in the AGATA experiment. A new state at $3780 \mathrm{keV}$ is tentatively assigned as the $3_{1}^{-}$state. The associated E3 strength is abnormally low as compared to RPA calculations. This trend observed in ${ }^{50,52} \mathrm{Ca}$ and ${ }^{54} \mathrm{Ti}$ is interpreted as a likely fragmentation of the low-energy octupole state (LEOS) below $6 \mathrm{MeV}$. The ${ }^{54} \mathrm{Ti}$ case evidence the complementary work realized with both tracking arrays AGATA and GRETINA.

\subsection{Lifetime measurements in $\mathrm{Zn}$ isotopes beyond the $N=40$ sub shell}

A different phenomenon than around $\mathrm{N}=32$ is at play around $\mathrm{N}=40$ and was evidenced by lifetime measurements in nuclei with $24<\mathrm{Z}<30$. The large excitation energy and small $\mathrm{B}(\mathrm{E} 2)$ value of the $2_{1}^{+}$state in ${ }^{68} \mathrm{Ni}$ are compatible with the presence of a sub-shell effect in this nucleus. However, these behaviors are related to the parity change induced by the $\nu f_{5 / 2}$ and $\nu g_{9 / 2}$ orbitals that constitute the $\mathrm{N}=40$ gap. By addition of only two neutrons, a large $\mathrm{B}(\mathrm{E} 2)$ value was established in ${ }^{70} \mathrm{Ni}$ [108]. Large-scale shell-model calculations involving a ${ }^{48} \mathrm{Ca}$ core, the $f p$-shell for protons and $\mathrm{pf}_{5 / 2} \mathrm{~g}_{9 / 2} \mathrm{~d}_{5 / 2}$ for neutrons were able to reproduce the experimental data [109] in the $\mathrm{Ni}$, Fe [110,111] and $\mathrm{Cr}$ isotopes [112,113] around $\mathrm{N}=40$ due to a sizeable contribution of $\nu g_{9 / 2}-v \mathrm{~d}_{5 / 2}$ excitations. The latter correlations induce collective behaviors at $\mathrm{N}=40$ in the above mentioned nuclei $(\mathrm{Z}<28)$ leading to a so-called island of inversion.

Two protons above $\mathrm{Ni}, \mathrm{B}\left(\mathrm{E} 2 ; 2_{1}^{+} \rightarrow \mathrm{O}_{1}^{+}\right)$values extracted from Coulomb excitations for the even-even ${ }^{74-80} \mathrm{Zn}$ isotopes performed at ISOLDE [114] indicate a maximum collectivity at $\mathrm{N}=44$ whereas a more recent lifetime measurement of the $2_{1}^{+}$state in ${ }^{74} \mathrm{Zn}$ leads to a lower value [115]. Other studies for the Se and Ge isotopes found a maximum collectivity at $\mathrm{N}=42$. Lifetime measurements for the $4_{1}^{+}$state bring additional information through the $\mathrm{B}_{4 / 2}=\mathrm{B}\left(\mathrm{E} 2 ; 4_{1}^{+} \rightarrow 2_{1}^{+}\right) / \mathrm{B}(\mathrm{E} 2$; $2_{1}^{+} \rightarrow 0_{1}^{+}$)ratio which is characteristic of the nuclear structure: $\mathrm{B}_{4 / 2}=2.0$ for harmonic vibration, 1.43 for rigid rotation and less then unity for non-collective nuclei close to shell closure.

The study of $\mathrm{Zn}$ isotopes above the $2_{1}^{+}$state and beyond $\mathrm{N}=40$, should help understanding the contribution of the $\nu \mathrm{d}_{5 / 2}$ orbital in their structure evolution. Therefore, an experiment has been run at LNL with the AGATA demonstrator composed of 3 ATCs and coupled to the PRISMA spectrometer placed at 55deg to explore the Zn structure with a focus on the lifetime measurement of the $2_{1}^{+}, 4_{1}^{+}$and possibly the $6_{1}^{+}$states of ${ }^{70,72,74} \mathrm{Zn}$ isotopes [116]. A ${ }^{76} \mathrm{Ge}$ beam at $577 \mathrm{MeV}$ impinged a ${ }^{238} \mathrm{U}$ target and the recoils passed through the Cologne plunger device holding the target and, downstream, a $\mathrm{Nb}$ foil used as degrader. Five target-degrader distances were used leading to a lifetime sensitivity in the 2 to $50 \mathrm{ps}$ range. The procedure to extract lifetime values was validated with the well-known lifetime of the $2_{1}^{+}$state in ${ }^{76} \mathrm{Ge}$. The influence of the side-feeding transitions of each state in $\mathrm{Zn}$ was studied in detail and, in the case of ${ }^{72} \mathrm{Zn}$, the use of the total kinetic energy obtained from PRISMA to select direct feeding of low-lying states enabled the suppression of high-spin paths feeding the $4_{1}^{+}$state. This technique, however, could not be used for the less populated ${ }^{70} \mathrm{Zn}$ and ${ }^{74} \mathrm{Zn}$ nuclei.

The present data fit perfectly the trend of the $\mathrm{B}\left(\mathrm{E} 2 ; 2_{1}^{+} \rightarrow 0_{1}^{+}\right.$) systematics for the $\mathrm{Zn}$ isotopes with $32<\mathrm{N}<50$ (upper left panel of Fig. 27). The three $\mathrm{B}\left(\mathrm{E} 2 ; 4_{1}^{+} \rightarrow 2_{1}^{+}\right.$) values from this work present a decreasing trend similar to the systematics, however shifted toward lower values which deserves discussion. An agreement with the previous data may be considered for ${ }^{70} \mathrm{Zn}$ considering the large error bars of the present data. For ${ }^{72} \mathrm{Zn}$, the $\mathrm{B}\left(\mathrm{E} 2 ; 4_{1}^{+} \rightarrow 2_{1}^{+}\right)$value is determined for the first time and is larger than the ones of the $\mathrm{Zn}$ isotopes with $\mathrm{N}<40$ which could be interpreted as partially due to a contribution of the two neutrons in the $g_{9 / 2}$ orbital. In the case of ${ }^{74} \mathrm{Zn}$, the $\mathrm{B}(\mathrm{E} 2)$ value obtained from the low-energy Coulomb-excitation experiment [114] is more than 4 times larger. This severe discrepancy could be explained by a long-lived state feeding the $4_{1}^{+}$state. However this transition is not present in the $\gamma$-ray spectra and no solid explanation is found.

The data were compared with beyond mean-field calculations using the Gogny D1S interaction in the 5DCH formalism $[117,118]$ and with two shell-model calculations using i) the JUN45 interaction with a ${ }^{56} \mathrm{Ni}$ core and the $\mathrm{pf}_{5 / 2} \mathrm{~g}_{9 / 2}$ valence space [119] and ii) the LNPS interaction with a ${ }^{48} \mathrm{Ca}$ core and the $\mathrm{pf}_{5 / 2} \mathrm{~g}_{9 / 2} \mathrm{~d}_{5 / 2}$ valence space [109]. The beyond mean-field calculations reproduce rather well the $2_{1}^{+}$and $4_{1}^{+}$excitation energies for $\mathrm{Zn}$ isotope with $\mathrm{N}<40$ but fail above and the calculated transition probabilities are systematically overestimated (left panel of Fig. 27). Both shell-model calculations are in agreement for the transition probabilities $\mathrm{B}\left(\mathrm{E} 2 ; 4_{1}^{+} \rightarrow 2_{1}^{+}\right.$) for ${ }^{70,72} \mathrm{Zn}$ but do not reproduce the very small experimental data for ${ }^{74} \mathrm{Zn}$ (lower left panel of Fig. 27). Indeed, the inclusion of the $n u \mathrm{~d}_{5 / 2}$ orbital in the LNPS 

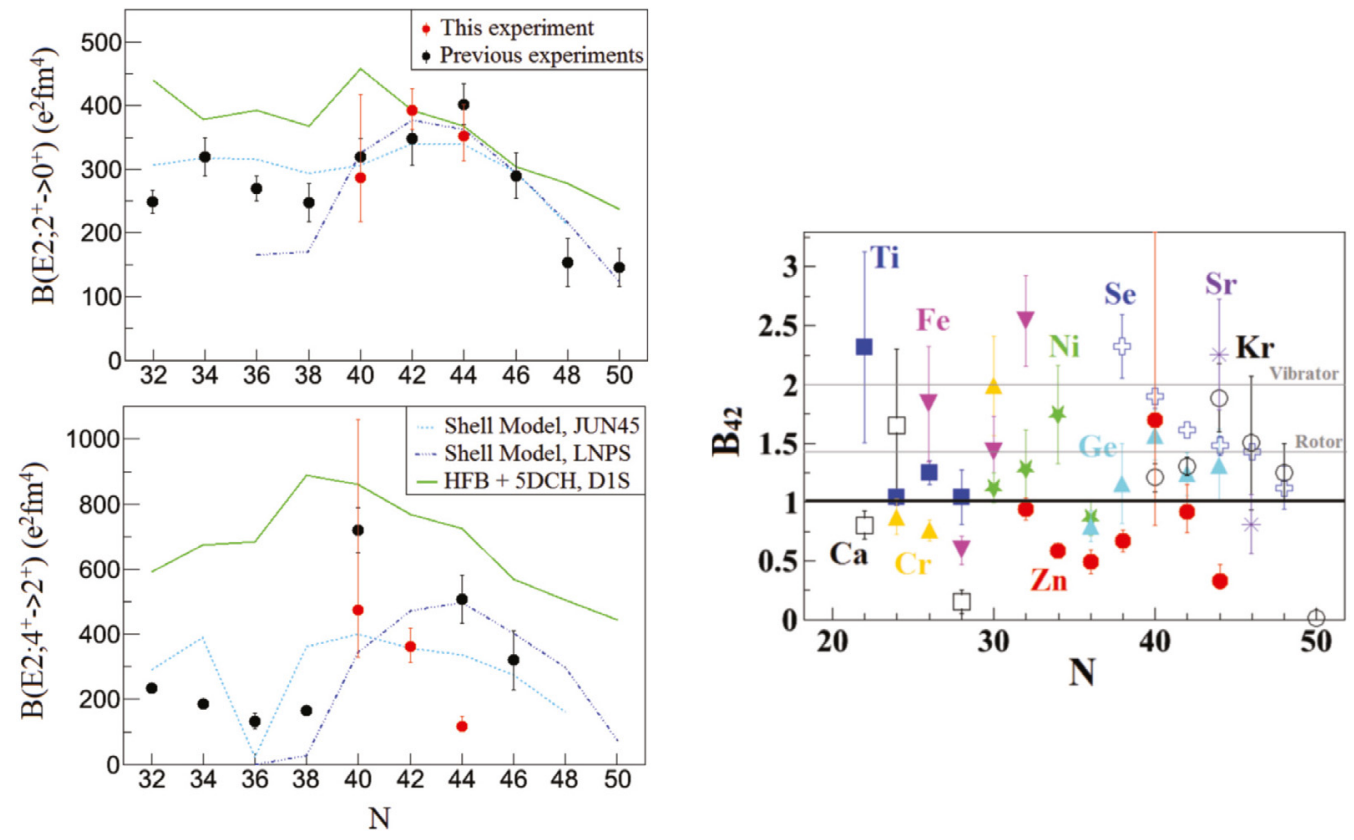

Fig. 27. Left panel: Systematics of experimental $\mathrm{B}\left(\mathrm{E} 2 ; 2_{1}^{+} \rightarrow 0_{1}^{+}\right)$and $\mathrm{B}\left(\mathrm{E} 2 ; 4_{1}^{+} \rightarrow 2_{1}^{+}\right)$values for the chain of $\mathrm{Zn}$ isotopes including the AGATA experimental data (red points). Theoretical values were calculated with the shell model using the JUN45 interaction (blue dotted line), the LNPS interaction (dark blue dashed-dotted line) and with a five-dimensional collective Hamiltonian using the Gogny D1S force. Right panel: Experimental $\mathrm{B}\left(\mathrm{E} 2 ; 4_{1}^{+} \rightarrow 2_{1}^{+}\right) / \mathrm{B}\left(\mathrm{E} 2 ; 2_{1}^{+} \rightarrow 0_{1}^{+}\right)$ratio for nuclei with $\mathrm{Z}=20$ to $\mathrm{Z}=40$. $\mathrm{Zn}$ isotopes are represented by red dots.

Source: Adapted from [116].

interaction enables a better fit of the $2_{1}^{+}$and $4_{1}^{+}$excitation energies and lead to an increase of the transition probabilities in contradiction with the experimental data of ${ }^{74} \mathrm{Zn}$.

Considering the experimental $\mathrm{B}_{4 / 2}=\mathrm{B}\left(\mathrm{E} 2 ; 4_{1}^{+} \rightarrow 2_{1}^{+}\right) / \mathrm{B}\left(\mathrm{E} 2 ; 2_{1}^{+} \rightarrow 0_{1}^{+}\right)$ratio presently available for $\mathrm{Ca}$ to $\mathrm{Kr}$ eveneven isotopes, the $\mathrm{Zn}$ isotopes present among the smallest $\mathrm{B}_{4 / 2}$ values, below one, which would indicate non-collective excitations (right panel of Fig. 27). This is typical when the seniority is a good quantum number and, as shown in the left panel of Fig. 27, it corresponds for $\mathrm{Zn}$ isotopes to a concomitant increase of $\mathrm{B}\left(\mathrm{E} 2 ; 2_{1}^{+} \rightarrow 0_{1}^{+}\right)$up to mid-shell with a decrease of $\mathrm{B}\left(\mathrm{E} 2 ; 4_{1}^{+} \rightarrow 2_{1}^{+}\right)$when adding neutrons in the $\mathrm{g}_{9 / 2}$ orbital $(\mathrm{N}>40)$.

These puzzling lifetime-measurement results call for further experimental and theoretical studies in neutron-rich $\mathrm{Zn}$ isotopes, in particular, for the $4_{1}^{+}$state in ${ }^{74} \mathrm{Zn}$ and for the heavier $\mathrm{Zn}$ isotopes.

\subsection{Structure evolution around $N=50$ shell closure}

Knowing that deformation appears in neutron-rich nuclei at the magic numbers $\mathrm{N}=20\left({ }^{32} \mathrm{Mg}\right), \mathrm{N}=28\left({ }^{44} \mathrm{~S}\right)$ and $\mathrm{N}=40\left({ }^{66} \mathrm{Fe}\right)$ leading to the so-called islands of inversion [100] and that ${ }^{132} \mathrm{Sn}$ is spherical [120], long-term efforts on both experimental and theoretical aspects concentrated more recently on the nature of ${ }^{78} \mathrm{Ni}$, an isotope 10 neutrons away from the heaviest stable $\mathrm{Ni}$ isotopes. Mass measurements in this region evidenced a minimum of the $\mathrm{N}=50$ shell gap at $Z=32$ [121,122]. Studying the structure of ${ }^{83,85}$ Se isotopes, Porquet et al. concluded to a $N=50$ gap quenching by $300 \mathrm{keV}$ for each proton pair removed from the fp shell [123]. On the contrary, [124-126] inferred the stability of the $\mathrm{N}=50$ gap towards ${ }^{78} \mathrm{Ni}$. The excitation energy of the $2^{+}$state and the $\mathrm{B}\left(\mathrm{E} 2 ; 2^{+} \rightarrow 0^{+}\right)$transition strength in ${ }^{80} \mathrm{Zn}$ showed a solid $\mathrm{N}=50$ gap in this nucleus and a strong $\mathrm{Z}=28$ core polarization [127]. Large-Scale Shell-Model (LSSM) calculations reproduce the $\mathrm{N}=50$ gap minimum at $\mathrm{Z}=32$ and predict a gap increase towards ${ }^{78} \mathrm{Ni}$ [128]. In 2019, the first spectroscopic information on the low-lying excited states of ${ }^{78} \mathrm{Ni}$ [129] was obtained. The excitation energy of the $2_{1}^{+}$state is $2.6 \mathrm{MeV}$ almost as high as for the $\mathrm{N}=28$ double magic ${ }^{56} \mathrm{Ni}$ isotope, confirming the magic character of ${ }^{78} \mathrm{Ni}$ which is spherical in its ground state. The $2_{2}^{+}$state is lying close to the first excited state at $2.91 \mathrm{MeV}$ and is interpreted as a deformed structure competing with the spherical one. Using several theoretical approaches, it is predicted that in the $\mathrm{Ni}$ isotopes beyond $\mathrm{N}=50$, the deformed structure becomes ground state $[100,129]$.

\subsubsection{The Se isotopes around $N=50$}

The structure evolution below and beyond $\mathrm{N}=50$ has been explored for $\mathrm{N}=46,48,50,52$ in ${ }^{80,82,84,86}$ Se isotopes probing the $\mathrm{N}=50$ gap and the main configurations in play. Detailed information may be obtained via lifetime measurements and comparison to Large-Scale Shell-Model results for a better understanding and interpretation of the 


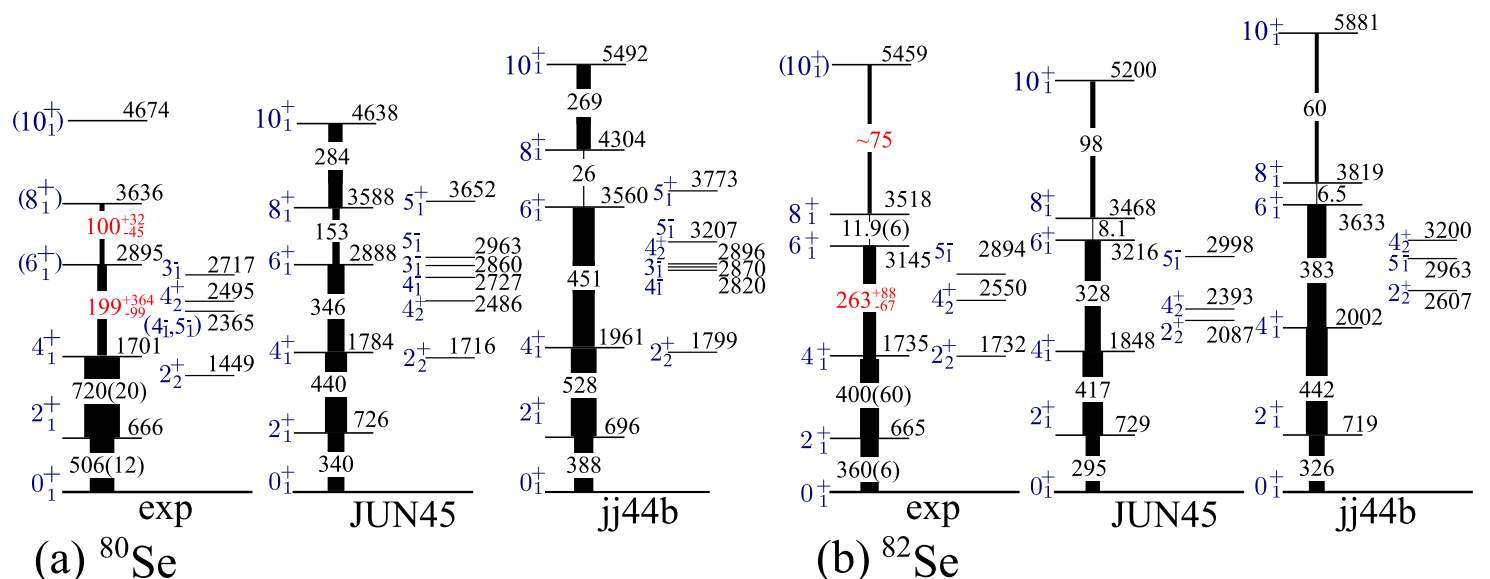

Fig. 28. Experimental yrast and selected sideband states of ${ }^{80,82}$ Se isotopes. Excitation energies and spin assignments are given as well as reduced transition probabilities (in $\mathrm{e}^{2} \mathrm{fm}^{4}$ ) for the yrast states. The new experimental B(E2) values from [134] are marked in red. For direct comparison, similar data resulting from LSSM calculations with JUN45 and jj44b effective interactions are also given.

Source: Taken from [134].

physics involved. In particular, a reduction of the $\mathrm{B}\left(\mathrm{E} 2 ; 8^{+} \rightarrow 6^{+}\right)$in ${ }^{80} \mathrm{Se}$ and more drastically in ${ }^{82}$ Se are observed. They are not reproduced by Large-Scale Shell-Model calculations performed with specific effective interactions, namely the JUN45 [119] and jj44b [130] ones. In the $N=50$ semi-magic nucleus ${ }^{84} \mathrm{Se}$, the excitation energy of the $6_{1}^{+}$state is lower compared to the one of the $5_{1}^{+}$state, a level arrangement not observed in other $\mathrm{N}=50$ nuclei. In ${ }^{86} \mathrm{Se}$ isotope, the $6_{1}^{+}$state excitation energy has been fixed at $2846 \mathrm{keV}[131,132]$ which leads to a large energy gap relative to the $4_{1}^{+}$state excitation energy. A similar behavior is also observed in neighboring $\mathrm{N}=52$ isotones and questions the nuclear configuration favoring such property. Lifetime measurements in these four neutron-rich Se isotopes could help understanding the physics at play around the $\mathrm{N}=50$ gap 6 protons away from ${ }^{78} \mathrm{Ni}$.

Lifetime measurements were performed at LNL with the AGATA demonstrator, the magnetic spectrometer PRISMA and the Cologne plunger. The Se isotopes were produced via multi-nucleon transfer using a ${ }^{82} \mathrm{Se}$ beam impinging onto a ${ }^{238} \mathrm{U}$ target. Transfer products were slowed down by a ${ }^{93} \mathrm{Nb}$ degrader and were detected in PRISMA. Three target-to-degrader distances were used 38(1), 257(2) and 507(7) $\mu \mathrm{m}$. Absolute distance information was extracted using well-known lifetimes in ${ }^{82} \mathrm{Se}$. The RDDS method was used for lifetime determination [63]. With recoil velocities of v/c $\approx 7 \%-9 \%$ for nuclei in the $A \sim 80$ mass region, lifetimes in the range of 1 ps to 100 ps were accessible. The limited statistics did not enable $\gamma \gamma$-coïncidence analysis. In case statistics are too limited to determine peak intensities for each distance individually, a new method using summed $\gamma$-ray spectra of different target-to-degrader distances was introduced [133]. Selection in the total kinetic energy losses (TKEL) helped reducing background and favoring high-lying states.

\section{${ }^{80,82}$ Se structure studies}

Lifetimes of levels of the yrast band and sidebands were measured in ${ }^{80,82} \mathrm{Se}$ [134]. Transition strengths are deduced for the first time for the $8_{1}^{+} \rightarrow 6_{1}^{+}$and $6_{1}^{+} \rightarrow 4_{1}^{+}$transitions in ${ }^{80}$ Se and for the $8_{1}^{+} \rightarrow 6_{1}^{+}$transition in ${ }^{82} \mathrm{Se}$. A lower limit for the $10_{1}^{+} \rightarrow 8_{1}^{+}$transition in ${ }^{82} \mathrm{Se}$ is also given (see Fig. 28). These experimental data are compared to LSSM calculations performed with the JUN45 and jj44b effective interactions. Both calculations reproduce the trend of the experimental results, theoretical results obtained with JUN45 being closer to experiment (see Fig. 28). The leading proton and neutron configurations coupling to the different spins of the yrast states up to $10^{+}$were extracted in ${ }^{80,82} \mathrm{Se}$. In both nuclei the $8_{1}^{+}$state is dominated $(\approx 50 \%)$ by a pure single-particle neutron in $g_{9 / 2}^{-2 /-4},\left(\nu 8^{+} \times \pi 0^{+}\right)$configuration. On the contrary, the $6_{1}^{+}$state contains only $\sim 20 \%$ of the $\nu 6^{+} \times \pi 0^{+}$configuration leading to a reduced $\mathrm{B}\left(\mathrm{E} 2 ; 8_{1}^{+} \rightarrow 6_{1}^{+}\right)$value relative to ${ }^{78} \mathrm{Se}$. In addition in ${ }^{82} \mathrm{Se}$, the second dominant $(\sim 35 \%)$ configuration of the $8_{1}^{+}$state is $\nu 8^{+} \times \pi 2^{+}$whereas the $\nu 6^{+} \times \pi 2^{+}$ configuration of the $6_{1}^{+}$state contains only $\sim 2 \%$. This gives rise to a strongly reduced $\mathrm{B}\left(\mathrm{E} 2 ; 8_{1}^{+} \rightarrow 6_{1}^{+}\right)$value in ${ }^{82} \mathrm{Se}$.

In ${ }^{80} \mathrm{Se}$, the proton and neutron configurations are more mixed with a clear difference between JUN45 and jj44b for the $8_{1}^{+}$state configurations. The $\mathrm{B}\left(\mathrm{E} 2 ; 8_{1}^{+} \rightarrow 6_{1}^{+}\right)$value with $\mathrm{jj} 44 \mathrm{~b}$ severely underestimates the experimental data whereas JUN45 is in better agreement. It is also observed that the LSSM calculations predict an overall evolution of the yrast-state $\mathrm{B}(\mathrm{E} 2)$ trend about inverse to the $\nu g_{9 / 2}$ configuration content of the decaying states. The structure of the $6_{1}^{+}$state in both nuclei exhibit a sizeable $\nu g_{9 / 2}$ contribution whereas the $4_{1}^{+}$state is dominated by proton collective excitations leading to reduced $\mathrm{B}\left(\mathrm{E} 2 ; 6_{1}^{+} \rightarrow 4_{1}^{+}\right)$values. The experimental trend is found to be rather well reproduced by the theoretical calculations. 


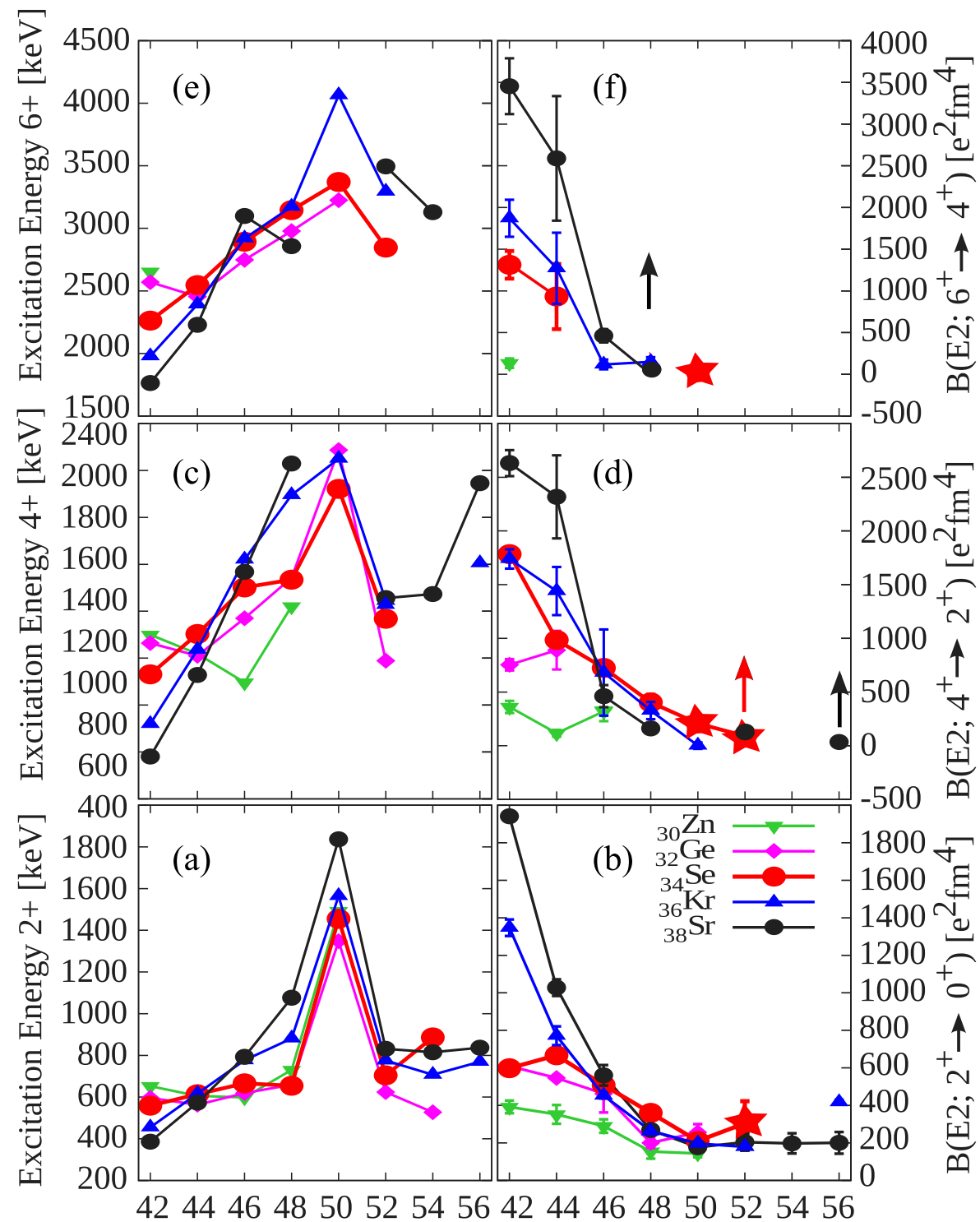

Neutron number

Fig. 29. Systematics of the $2_{1}^{+}, 4_{1}^{+}$and $6_{1}^{+}$state excitation energies [(a),(c) and (e)] and corresponding B(E2) values [(b), (d) and (f)] for Se and neighboring isotopic chains with $\mathrm{N}=42-56$. The stars denoted as "this work" are the data from [133].

Source: Taken from [133].

This work shows a progressive evolution in Se isotopes of the $\mathrm{B}(\mathrm{E} 2)$ strength from collective in ${ }^{78}$ Se to dominantly single particle close to the shell closure as expected. It underlines also the major impact of the single-particle $\left(\nu g_{9 / 2}\right)^{-2 /-4}$ configurations for the proper description of both $8_{1}^{+}$and $6_{1}^{+}$state decays in ${ }^{80,82} \mathrm{Se}$.

${ }^{84,86}$ Se structure studies

In the $\mathrm{N}=50(52)^{84(86)}$ Se nucleus, $4_{1}^{+}\left(2_{1}^{+}\right)$and $6_{1}^{+}\left(4_{1}^{+}\right)$state lifetimes or effective lifetimes were determined for the first time and the deduced B(E2) values fit pretty well the systematics of Se isotopes and $Z=32-38$ isotones [133] (see red stars in Fig. 29). For ${ }^{84} \mathrm{Se}$, comparison of the experimental data with LSSM calculation using a ${ }^{56} \mathrm{Ni}$ inert core with the proton $f_{5 / 2} p_{3 / 2}$ valence space and the Ni78-II [132], the JUN45 [119], the jj44b and the jj4pna [130] effective interactions was performed. An overall agreement is obtained except for the abnormally large experimental $\mathrm{B}\left(\mathrm{E} 2 ; 4_{1}^{+} \rightarrow 2_{1}^{+}\right)$value. Discrepancies of up to three orders of magnitude are observed. A detailed analysis evidence the important contribution of the $p_{1 / 2}$ orbital to the calculated transition strength via the proton $f_{5 / 2} \rightarrow p_{1 / 2}$ stretched E2 transition. It is pointed out that inclusion of other excitations such as proton $\pi f_{7 / 2} \rightarrow \pi p_{3 / 2}$ or neutron $v g_{9 / 2} \rightarrow \nu d_{5 / 2}$ would also enhance the E2 strength. 
It is noteworthy to mention that $5_{1}^{+}, 6_{2}^{+}$and $7_{1}^{+}$state excitation energies which are mainly built on neutron single-particle configurations are significantly lower in ${ }^{84} \mathrm{Se}$ than in heavier neighbors indicating that neutron-core excitations should be taken into account in this nucleus. The spin assignment of the $6_{1}^{+}$state located at $3370 \mathrm{keV}$ excitation energy is supported by the theoretical calculations.

For ${ }^{86} \mathrm{Se}$, LSSM calculations were performed with a ${ }^{78} \mathrm{Ni}$ inert core and a large valence space for both protons and neutrons. The Ni78-I [131,135] and Ni78-II [132] effective interactions were used. For the $0_{1}^{+}, 2_{1}^{+}, 4_{1}^{+}$and $6_{1}^{+}$states, Ni78-I predicts a main contribution of the $\pi f_{5 / 2} p_{3 / 2} \times v d_{5 / 2}$ configuration and a negligible occupation of the $v g_{7 / 2}$ orbital. A good agreement between the experimental and theoretical $\mathrm{B}\left(\mathrm{E} 2 ; 2_{1}^{+} \rightarrow 0_{1}^{+}\right)$values is obtained and the lower limit of the experimental $\mathrm{B}\left(\mathrm{E} 2 ; 4_{1}^{+} \rightarrow 2_{1}^{+}\right.$) transition probability is compatible with theory. The Ni78-I calculations predict a fairly constant deformation $\beta \approx 0.2$ along the ground-state band from $0_{1}^{+}$to $6_{1}^{+}$states with a constant $\gamma$ parameter $\gamma \approx 20^{0}$ up to the $4_{1}^{+}$state and a reduced value $\left(\gamma=12^{0}\right)$ for the $6_{1}^{+}$state. The latter results complement those obtained by Lettmann et al. [136] in the neutron-rich Ge isotopes with $\mathrm{N}>50$. These Ge and Se nuclei seem to develop triaxial structures, with some degree of rigidity for the Ge ground states, in particular in ${ }^{86} \mathrm{Ge}$, and triaxial susceptibility for the Se isotopes.

\subsection{2. $\mathrm{N}=51$ nuclei towards ${ }^{78} \mathrm{Ni}$}

Spectroscopic information become scarce when approaching $Z=28$ for $N>50$ nuclei. In $N=51$ nuclei from Sn down to $\mathrm{Ge}$, the $5 / 2^{+}$ground state corresponds to the single valence neutron in the $v 2 d_{5 / 2}$ orbital [137]. The first $1 / 2^{+}$excited state dominated by the $v 3 s_{1 / 2}$ configuration is down sloping from $\mathrm{Zr}$ to $\mathrm{Ge}$. The evolution versus $\mathrm{N}$ of the other orbitals $v 2 d_{3 / 2}, v 1 g_{7 / 2}$ and $v 1 h_{11 / 2}$ is not yet determined. In this context the placement of the $v 1 g_{7 / 2}$ orbital is of importance.

Previous studies established that $7 / 2^{+}$states in ${ }^{87} \mathrm{Kr}[138,139]$ and in ${ }^{85} \mathrm{Se}[140]$ are located at $2520 \mathrm{keV}$ and $1115 \mathrm{keV}$ excitation energy, respectively, and have large spectroscopic factors $S=0.49$ and $S=0.77$, respectively. This implies that the single-particle energy of the $v 1 g_{7 / 2}$ orbital dramatically drops by about $1.4 \mathrm{MeV}$ by removing one proton pair from ${ }^{87} \mathrm{Kr}$. However, in the latter nucleus, a first excited $7 / 2^{+}$state is lying at $1419 \mathrm{keV}$ excitation energy with a very small spectroscopic factor. It could correspond to a collective, vibrational state coupling the single-valence neutron in $v 2 d_{5 / 2}$ orbital to the $2^{+}$excitation of the ${ }^{86} \mathrm{Kr}$ core. Such coupling generates a multiplet of $1 / 2^{+}$to $9 / 2^{+}$states with the $7 / 2^{+}$ state being energetically the most favored. A simple coupling scheme predicts states with short lifetimes (below $1 \mathrm{ps}$ ) whereas single-particle state lifetimes would be expected in the range of several tens of ps.

In order to unravel the imbroglio on the structure of the $7 / 2^{+}$states in ${ }^{87} \mathrm{Kr}$ and ${ }^{85} \mathrm{Se}$ nuclei, lifetime measurements were performed at LNL with the same AGATA experiment as for the Se isotopes discussed above. The goal was to determine whether the lifetimes of $7 / 2^{+}$and possibly other states were short or long to fix the single-particle or vibrational, collective configuration character of the states of interest [141]. Two target-to-degrader distances of $38 \mu \mathrm{m}$ and $257 \mu \mathrm{m}$ were used in this analysis. Two side-feeding effective lifetimes were considered, very short ones $(<0.1 \mathrm{ps})$ and lifetimes corresponding to the average effective lifetimes observed in the discrete transitions of ${ }^{87} \mathrm{Kr}$ and ${ }^{85} \mathrm{Se}$ nuclei. Intrinsic lifetime values were obtained for the first $7 / 2^{+}$and $9 / 2^{+}$states in ${ }^{87} \mathrm{Kr}$ which are short $(\tau=0.4+1.6 /-0.4 \mathrm{ps}$ and $<0.1 \mathrm{ps}$, respectively) and characteristic of the core-coupled $2^{+} \times v 2 d_{5 / 2}$ scheme. Effective lifetimes, not accounting for side-feeding thus overestimating the intrinsic lifetimes, were extracted for the first $7 / 2^{+}$and $9 / 2^{+}$states in ${ }^{85}$ Se (see Fig. 30). Upper limits of 3(2) ps were obtained leading to the same conclusion [141] in contradiction with [140]. These results pull the $v 1 g_{7 / 2}$ orbital to higher single-particle energies which is compatible with Litzinger et al. whose calculations do not involve the $v g_{7 / 2}$ orbital up to the $6_{1}^{+}$state configuration, states lying at $2895 \mathrm{keV}$ and $3145 \mathrm{keV}$ excitation energy in ${ }^{84} \mathrm{Se}$ and ${ }^{86} \mathrm{Se}$, respectively [133]. Further lifetime studies in lighter $N=51$ isotones $\left({ }^{83} \mathrm{Ge}\right.$ and $\left.{ }^{81} \mathrm{Zn}\right)$, would enable to identify states dominated by single-particle configurations close to ${ }^{78} \mathrm{Ni}$ beyond the $\mathrm{N}=50$ gap and contribute to the determination of their corresponding effective single-particle energies.

\subsection{3. $N=50$ core excitations in ${ }^{81}$ Ga nucleus}

Direct spectroscopy of low-lying states in the hard to reach ${ }^{78} \mathrm{Ni}$ has been recently published [129]. Two structures are evidenced, the ground-state band and an yrare band. These structures are interpreted as corresponding to spherical states and coexisting collective states, respectively [142]. All ${ }^{78} \mathrm{Ni}$ excited states involve both proton and neutron core excitations across the $Z=28$ and $N=50$ gaps which have similar sizes. Whereas the spherical states are mainly built on single particle-hole excitations ( $1 \mathrm{p}-1 \mathrm{~h}$ ), the collective states are based on np-nh with $n \geq 4$. In the $Z>28, N=50$, easier to reach, ${ }^{80} \mathrm{Zn}$ and ${ }^{81} \mathrm{Ga}$ nuclei with two and three valence protons located in the fp shell, respectively, states with maximum spin and parity $4^{+}$and $11 / 2^{-}$can be built. Higher spin states in these two nuclei give access to the coupling of valence protons to particle-hole core excitations that deserve further studies.

Neutron-rich nuclei were produced at GANIL in fusion-fission reactions using a ${ }^{238} \mathrm{U}$ beam impinging on a thick ${ }^{9} \mathrm{Be}$ target [143]. The ${ }^{81} \mathrm{Ga}$ fragments, the lightest $\mathrm{N}=50$ nucleus accessible in these data for gamma-ray spectroscopy, were isotopically identified in the large acceptance VAMOS++ magnetic spectrometer and the associated prompt gamma-rays were detected in AGATA (see Section 2.4). Three new transitions of $327 \mathrm{keV}, 813.6 \mathrm{keV}$ and $1398.5 \mathrm{keV}$ were observed and none of the transitions reported in [126] could be confirmed. As it is commonly agreed that fission reactions mainly populate high-spin yrast states, spin and parity assignments were made accordingly.

Low-lying states develop from a $5 / 2^{-}$ground state up to the $\left(11 / 2^{-}\right)$state. The $327 \mathrm{keV}$ and $813.6 \mathrm{keV}$ transitions which are in direct coincidence with the $611.5 \mathrm{keV}$ and the $1340.7 \mathrm{keV}$ low-lying transitions are placed higher in the level scheme. They are suggested to de-excite $\left(15 / 2^{-}\right)$and $\left(13 / 2^{-}\right)$states (see Fig. 31 ). LSSM calculations using the PFSDG-U 

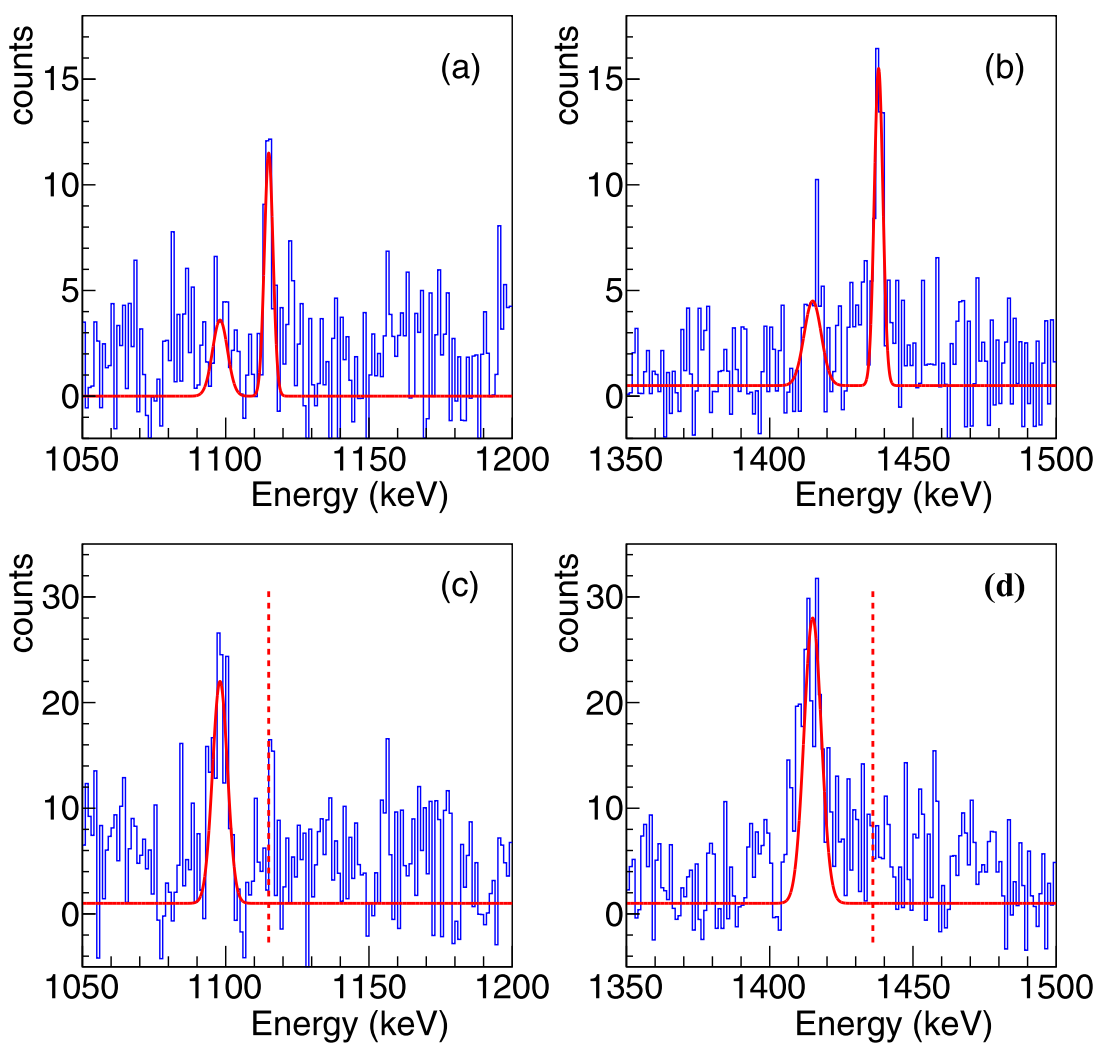

Fig. 30. Left panel Background-subtracted $\gamma$-ray spectra of ${ }^{85} \mathrm{Se}$ for a target-to-degrader distances of (a), (b) $38 \mu \mathrm{m}$ and (c), (d) $257 \mu \mathrm{m}$. The Gaussian fits of the shifted and unshifted peak components are shown in red for the (a), (c) $7 / 2^{+} \rightarrow 5 / 2_{\text {g.s. }}^{+}$and (b), (d) $\left(9 / 2^{+}\right) \rightarrow 5 / 2_{\text {g.s. }}^{+}$transitions at 1115 and $1436 \mathrm{keV}$, respectively. In panels (c) and (d) the dashed lines show the position of the unshifted peak at the $257 \mu \mathrm{m}$ distance. Source: Adapted from [141].

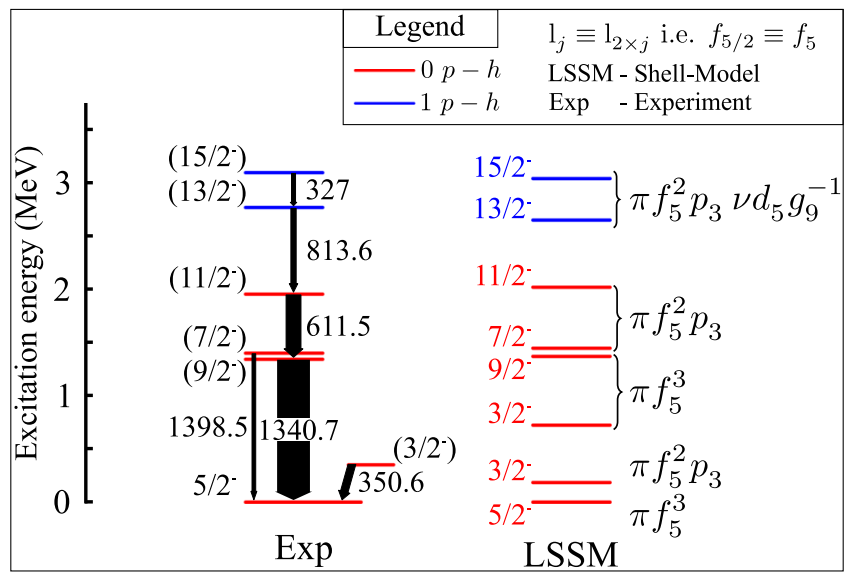

Fig. 31. Left panel: Experimental level scheme of ${ }^{81} \mathrm{Ga}$ [143]. Right panel: Theoretical level scheme deduced from LSSM using the PFSDG-U interaction [142]. States marked in red(blue) correspond to $0 \mathrm{p}-0 \mathrm{~h}(1 \mathrm{p}-1 \mathrm{~h})$ excitations in ${ }^{78} \mathrm{Ni}$ core.

Source: Adapted from [143].

interaction [142] were performed with a ${ }^{60} \mathrm{Ca}$ inert core and a large valence space for both protons (pf orbitals) and neutrons (sdg orbitals). The low-lying states from $5 / 2^{-}$up to $11 / 2^{-}$correspond mainly to the coupling of the valence protons occupying the $\mathrm{f}_{5 / 2}, \mathrm{p}_{3 / 2}$ and $\mathrm{p}_{1 / 2}$ orbitals (0p-0h core excitations). For the higher lying $13 / 2^{-}$and $15 / 2^{-}$states, the coupling of the $\pi \mathrm{f}_{5 / 2}^{2} \mathrm{p}_{3 / 2}$ proton configuration with the $\nu \mathrm{d}_{5 / 2} \mathrm{~g}_{7 / 2}^{-1} 1 \mathrm{p}-1 \mathrm{~h}$ neutron excitation is dominant. The agreement between experiment and theory is excellent as shown in Fig. 31. 

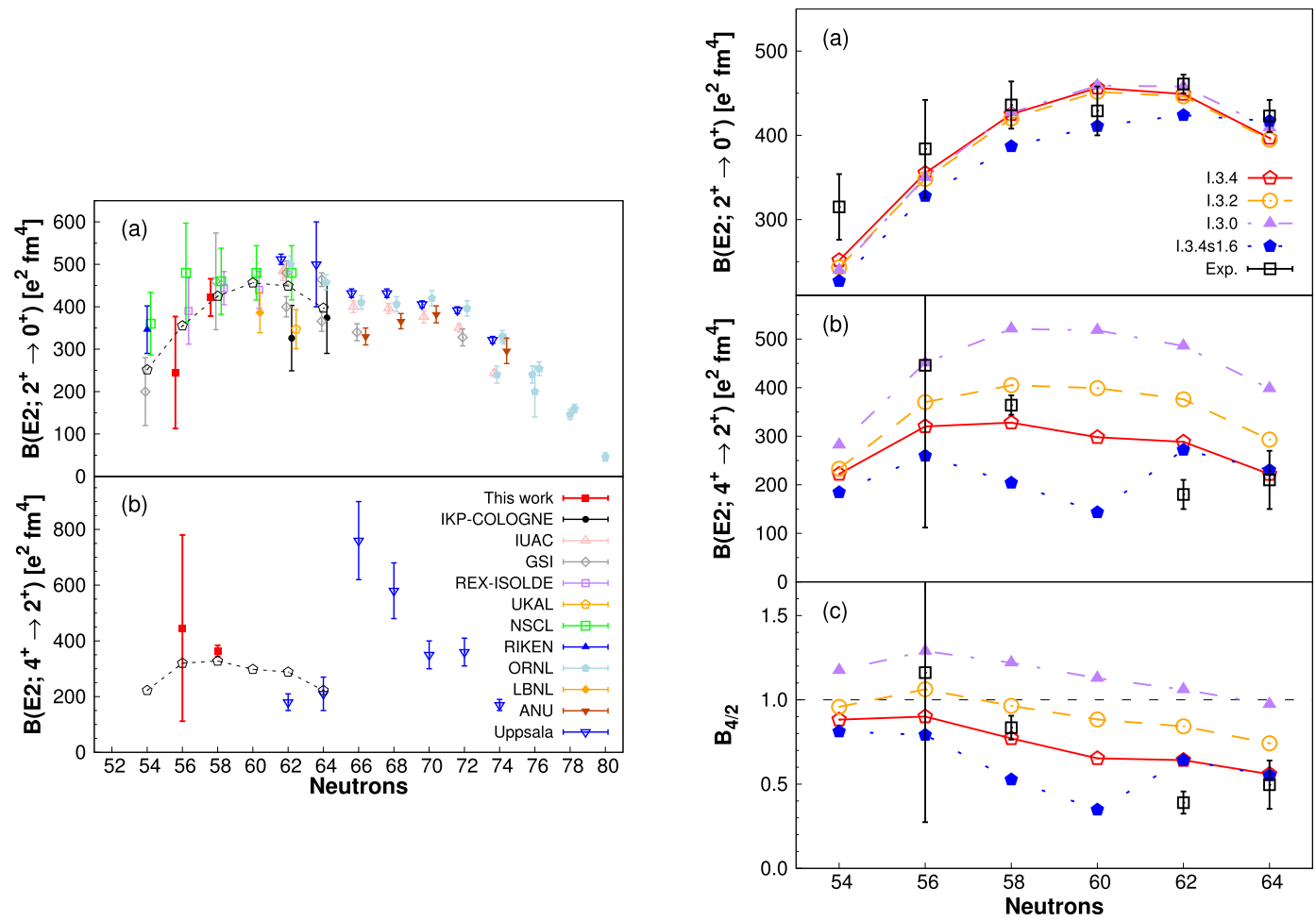

Fig. 32. Left panel: Reduced transition probabilities $\mathrm{B}\left(\mathrm{E} 2 ; 2_{1}^{+} \rightarrow 0_{1}^{+}\right)(\mathrm{a})$ and $\mathrm{B}\left(\mathrm{E} 2 ; 4_{1}^{+} \rightarrow 2_{1}^{+}\right)$(b) along the Sn isotopic chain. The predictions from LSSM calculations using the I.3.4 interaction, which includes 40\% pairing strength correction (see text and [145] for more details) are depicted by open black pentagons. Right panel: Experimental (open black squares) and theoretical B(E2; $2_{1}^{+} \rightarrow 0_{1}^{+}$) (a) and $\mathrm{B}\left(\mathrm{E} 2 ; 4_{1}^{+} \rightarrow 2_{1}^{+}\right)(\mathrm{b})$ values and $\mathrm{B}_{4 / 2}$ ratio. Effects of the pairing strength $\left(0 \%, 20 \%\right.$ and $40 \%$ for interactions I.3.0, I.3.2 and I.3.4, respectively) and of the placement of the $s_{1 / 2}$ orbital (I.3.4s1.6) are discussed in [145]. Experimental B(E2; $2_{1}^{+} \rightarrow 0_{1}^{+}$) values are the weighted averages of Se-Zn results given in the left panel (b) while $\mathrm{B}\left(\mathrm{E} 2 ; 4_{1}^{+} \rightarrow 2_{1}^{+}\right.$) values are taken from [144] and [145]

Source: Adapted from [145].

A good approximation of the effective $\mathrm{N}=50$ shell gap (the gap altered by the correlations) may be obtained from the difference of the one-neutron separation energies for $30<Z<34$ from experimental and theoretical calculations with $\Delta_{n}=S_{n}(Z, N=50)-S_{n}(Z, N=51)$. The $\Delta_{n}$ values are compared to the excitation energies of the $5^{+}$and $6^{+}$states in even and $13 / 2^{-}$and $15 / 2^{-}$states in odd nuclei. A good agreement is found which indicates that the main configuration of these high-lying states are $\mathrm{p}-\mathrm{h}$ neutron excitations across the $\mathrm{N}=50$ gap. The $\Delta \mathrm{n}$ values exhibit a decreasing trend from $Z=34$ down to $Z=31$ and the calculated values show a rapid increase below the $Z=31$ minimum towards $Z=$ 28 , suggesting a strong $\mathrm{N}=50$ shell gap in ${ }^{78} \mathrm{Ni}$. The comparison of the state occupancy in ${ }^{78} \mathrm{Ni}$ and ${ }^{81} \mathrm{Ga}$ suggests that ${ }^{81} \mathrm{Ga}$ states correspond to the coupling of the three valence protons to the ${ }^{78} \mathrm{Ni}$ core and its high-spin states to $1 \mathrm{p}-1 \mathrm{~h}{ }^{78} \mathrm{Ni}$ core excitations (see Fig. 31). This study supports the doubly magic character of ${ }^{78} \mathrm{Ni}$ and its persistence for $\mathrm{Z}>28, \mathrm{~N}=$ 50 isotones.

\subsection{Structure evolution in the neutron-deficient semi-magic tin isotopes}

Up to now, the Sn isotopic chain was considered as a text book case of isovector $T=1$ pairing dominance with, in particular, a parabolic behavior of the $\mathrm{B}\left(\mathrm{E} 2 ; 2_{1}^{+} \rightarrow 0_{1}^{+}\right)$versus neutron number. However, the latter property validated for $A>116$ is replaced by rather constant $B(E 2)$ values for lower masses (see left panel in Fig. 32). Experimental data for $\mathrm{B}\left(\mathrm{E} 2 ; 4_{1}^{+} \rightarrow 2_{1}^{+}\right.$) only exist for ${ }^{112,114} \mathrm{Sn}[144]$.

This motivated lifetime measurements at GANIL for both the $2_{1}^{+}$and $4_{1}^{+}$states in ${ }^{106,108} \mathrm{Sn}$ isotopes using the RDDS technique with a differential plunger. A multi-nucleon transfer reaction, generally used to populate neutron-rich nuclei, has been applied for this neutron-deficient case with a ${ }^{106} \mathrm{Cd}$ beam colliding a ${ }^{92} \mathrm{Mo}$ target. A natural $\mathrm{Mg}$ degrader foil was used to slow down the transfer products which were detected and isotopically identified in VAMOS++ placed at the grazing angle. The total kinetic energy loss TKEL was calculated and gates were placed to select the state population avoiding long-lived components from the isomeric $6_{1}^{+}$state. Gamma rays were detected with AGATA in a compact geometry. Eight target-to-degrader distances ( 31 to $521 \mu \mathrm{m}$ ) were used to measure the $4_{1}^{+}$and $2_{1}^{+}$state lifetimes for the first time in ${ }^{106,108} \mathrm{Sn}$ isotopes [145]. The $\mathrm{B}\left(\mathrm{E} 2 ; 2_{1}^{+} \rightarrow 0_{1}^{+}\right.$) values (red squares) agree fairly well with the existing data (see upper left 
panel in Fig. 32). A very precise point for B(E2; $4_{1}^{+} \rightarrow 2_{1}^{+}$) in ${ }^{108}$ Sn was obtained (see lower left panel in Fig. 32), the data in ${ }^{106} \mathrm{Sn}$ having large error bars.

LSSM calculations were performed with the N3LO realistic potential [146], low-k potential correction [147], renormalized quadrupole and phenomenological pairing forces [148] and replaced monopole part of the interaction [149]. The resulting interactions with $0 \%, 20 \%$ and $40 \%$ pairing strength correction are called I.3.0., I.3.2 and I.3.4, respectively. An adjustment by $800 \mathrm{keV}$ of the $s_{1 / 2}$ orbital leads to the I.3.4s1.6 interaction. The right panel of Fig. 32 shows that the intensity of pairing correction has no impact on the calculated $B\left(E 2 ; 2_{1}^{+} \rightarrow 0_{1}^{+}\right.$) values (Fig. 32(a)) except when the $s_{1 / 2}$ orbital is shifted. On the contrary, the theoretical $\mathrm{B}\left(\mathrm{E} 2 ; 4_{1}^{+} \rightarrow 2_{1}^{+}\right.$) values are strongly affected by pairing. The precise measurement performed for ${ }^{108} \mathrm{Sn}$ reported in [145] enables, without any ambiguity, to fix the pairing correction to $40 \%$ and to give insight to the ${ }^{101} \mathrm{Sn}$ spectrum.

In conclusion, while the pairing was considered dominating the $\mathrm{Sn}$ isotopic chain, the experimental results and associated LSSM calculations presented by [145] indicate that the plateau of $\mathrm{B}\left(\mathrm{E} 2 ; 2_{1}^{+} \rightarrow 0_{1}^{+}\right)$values is induced by quadrupole correlations independently of the pairing strength. For the $4_{1}^{+}$state, a strong quadrupole-pairing balance is invoked related to mixing with a pairing dominated intruder. To elucidate this question further work is needed with larger statistics in the same isotopes and closer to the doubly-magic ${ }^{100} \mathrm{Sn}$ isotope.

\section{Collective excitations}

Starting from the earliest investigations of nuclear excitation properties it appeared clear that nuclei are very complex systems characterized by the presence of both single-particle excitations and collective modes and by their interplay. It is well known that the description of these modes, in terms of nucleon configurations and their underlying interactions, requires the knowledge of the electromagnetic observables and thus these basic concepts are presently much exploited, particularly for nuclei poorly known and/or for excitations and decays not easily experimentally accessible but very interesting for testing modern theories.

In connections with the investigation of nuclear collective modes a number of experiments were made with the AGATA array addressing key questions on the following topics: (i) low-lying quadrupole and octupole type vibrations and their couplings to single particles; (ii) the low-lying dipole excitation in stable and unstable nuclei; (iii) Giant Dipole Resonance (GDR) excitation in hot rotating nuclei, damping mechanisms underlying its width; (iv) the probe of isospin symmetry at high excitation energy.

\subsection{Quadrupole and octupole excitations and couplings to single particles}

The idea that there are specific "building blocks", namely single-particle excitations and collective modes, that can be used to describe nuclear excitations has proven to be very instrumental for the progress in the study of nuclear structure. In fact, the shell model cannot always predict vibrational or rotational states (and other collective phenomena such as clustering in nuclei) and thus it became rather natural to perceive that several nuclear excited states can be described in terms of single particle excitations and collective modes and of their combinations.

The existing experimental work, performed often in parallel with increasingly more refined theoretical studies, has confirmed the relevance of the picture of atomic nuclei based on the interplay between fermionic and bosonic degrees of freedom. In this context, odd nuclei are of special interest, being sensitive to terms in the nuclear force (time-odd terms) and to correlations that simply do not show up in even-even systems. From a broader perspective, coupling phenomena between fermions and collective excitations (bosons) are well known to occur in many-body systems in all branches of physics. Starting from the emblematic cases in electronic systems: the electron-plasmon couplings in metals, or the electron-phonon couplings that give rise to superconductivity. These phenomena are the counterparts of the couplings of nucleons with collective excitations. In addition, in contrast to molecules, the nuclear energy scales related to vibrational and single-particle excitations are of the same order, and thus their interweaving has profound consequences.

The most recent work devoted to the understanding of the interplay between collective and single-particle degrees of freedom, which was carried out with AGATA, was focused on moderate neutron-rich nuclei in the region of around the Ca nucleus $(Z=20)$ and Ni nucleus $(Z=28)$ as schematically illustrated in Fig. 33.

Related questions are concerned with the problem of shape coexistence in nuclei, characterized by the existence of two or more states with spin 0 and positive parity. The crucial point is to establish the deformation of these $0^{+}$states by determining the value of the different quadrupole moments on which excited states, such as rotational bands, can be built on. In this connection the ${ }^{42} \mathrm{Ca}$ nucleus with only two nucleons outside the doubly magic ${ }^{40} \mathrm{Ca}$ core is, according to theory, an intriguing case to study experimentally. Details on this investigation and results are described in the next chapter related to nuclear shapes (see chapter 6 Section 6.1).

It is important to recall here that for the neutron-rich $\mathrm{Ni}$ isotopes of even mass there is a rather rich information collected so far. These nuclei have attracted attention in recent years because of the occurrence of shape or configuration coexistence [150-156]. The neutron single-particle strengths at $\mathrm{N}=40$ and 42 were extracted from neutron knockout measurements from ground states and isomeric states with an experiment with GRETINA [157]. For the ${ }^{70} \mathrm{Ni}$ nucleus evidence of an enhanced dipole strength below the binding energy [158] was found. In addition for this nucleus, the role of neutron and proton configurations in excited states was investigated by measuring the difference in population of excited final states via proton and neutron knock-out reactions [159]. The first $2^{+}$state appears to have as a leading configuration proton excitations across the $Z=28$ shell closure. Such states are suggested in the literature to form a collective structure associated with prolate deformation. 


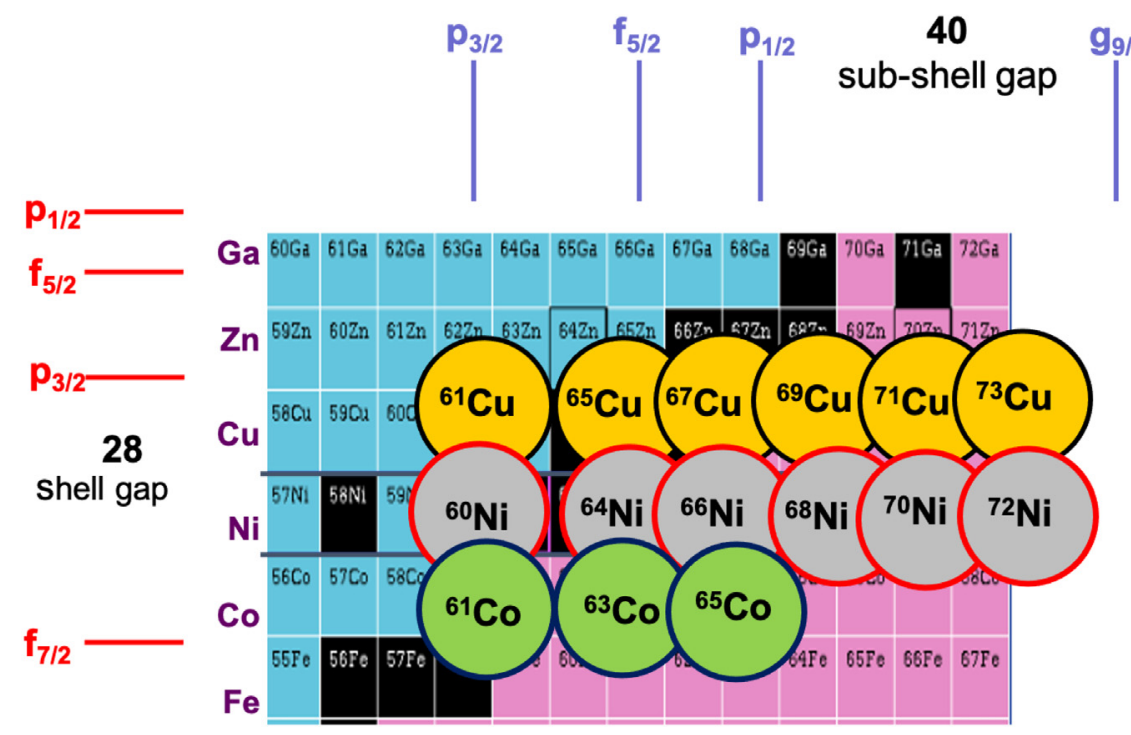

Fig. 33. Schematic illustrations of the single-particle orbitals of the levels involved in the structures of the neutron-rich Cu,Ni,Co nuclei discussed in this paper. The red scheme on the left is for protons and the blue vertical bars are the levels for neutrons.

\subsubsection{Coupling to core excitations in odd $\mathrm{Cu}$ and $\mathrm{Co}$ nuclei}

The $\mathrm{Cu}$ isotopes, having one proton outside the $\mathrm{Z}=28$ shell gap, have attracted interest since for these nuclei the excitation spectra could be rather complex and characterized by the presence, among others, of states in which the proton couples to the excitation of the core.

Coulomb excitation is known to be an ideal probe for exciting collective modes in even-even nuclei and thus it is largely employed particularly at low beam energy below Coulomb Barrier to populate both low-lying quadrupole and octupole vibrational states and rotational states in deformed nuclei. To study unstable neutron-rich odd-even ${ }^{67,69,71,73} \mathrm{Cu}$ isotopes Coulomb excitation in inverse kinematics was employed [160].

Candidates for collective excitations were identified in this experiment [160] since large $B(E 2)$ values were measured (see Fig. 34). In particular, for the second $7 / 2^{-}$state in all odd $\mathrm{Cu}$ isotopes the $\mathrm{B}\left(\mathrm{E} 2 ; 7 / 2^{-} \rightarrow 3 / 2^{-}\right.$) values and the excitation energy were found to follow very well those of the $2^{+}$state in the corresponding Ni cores. This can be seen in the two right panels of Fig. 34. Based on the particle-core model [161], the $7 / 2^{-}$states in ${ }^{71,73} \mathrm{Cu}$ at 1190 and $961 \mathrm{keV}$, respectively, were interpreted as $\pi 2 \mathrm{p}_{3 / 2} \times 2^{+}\left({ }^{70,72} \mathrm{Ni}\right)$ configurations, while the $\pi 1 \mathrm{f}_{7 / 2}^{-1} 2 \mathrm{p}_{3 / 2}^{2}$ structure was assigned to the $7 / 2^{-}$levels located at 981 and $1010 \mathrm{keV}$, respectively [161]. The non observation of the latter 7/2- states (lying at lower energy in $\left.{ }^{71} \mathrm{Cu}\right)$ in a Coulomb excitation experiment and the obtained $\mathrm{B}(\mathrm{E} 2)$ values for the former, very similar to the $\mathrm{B}(\mathrm{E} 2)$ strengths in the corresponding $\mathrm{Ni}$ cores, is consistent with the proposed interpretation. Therefore, also for the second $7 / 2^{-}$state in lighter $\mathrm{Cu}$ isotopes, having the same properties, one can easily extend this interpretation for their structure (see right panels of Fig. 34).

The picture obtained with the Coulomb excitation data for the odd isotopes of $\mathrm{Cu}$ indicates that some levels can be described as coupling to core collective excitations but others, although with the same spin value, appear to have a different structure involving single-particle excitation of the core. To resolve this picture lifetime measurements were made with AGATA using a reaction which shows an enhanced population of the 7/2- state compared to Coulomb excitation [162]. Indeed three low-lying $7 / 2^{-}$states can emerge in neutron-rich $\mathrm{Cu}$ isotopes from single-proton excitations above the $Z=28$ gap or from the coupling of a proton in the $\pi 2 p_{3 / 2}$ or $\pi 1 f_{5 / 2}$ orbital to the $2_{1}^{+}$state of the even-even Ni core. To understand their structure one needs to measure the electromagnetic transition $B(E 2)$ using the gamma-rays of quadrupole character to the $3 / 2^{-}$ground state. The multi-nucleon transfer reaction ${ }^{76} \mathrm{Ge}+{ }^{238} \mathrm{U}$ at a bombarding energy of $577 \mathrm{MeV}$ was employed in an experiment performed at the laboratory LNL with the configuration described in Section 2.2. The plunger device was used to measure lifetimes exploiting the Recoil Distance Doppler Shift method (RDDS).

Two of the measured $R$ curves to derive the lifetimes are shown in the left panels of Fig. 35 and their values are reported together with the other obtained values and with predictions in the table given in the right panel of Fig. 35. These lifetimes were deduced from the ratios of counts $R(d)=I_{S}(d) / N_{A}(d)$ at each distance $d$ between the target and stopper foil. In this expression $I_{S}$ is the number of counts in the peak corresponding to the decay after the degrader and $\mathrm{N}_{A}$ is the total number of nuclei measured at the PRISMA focal plane. The distance $\mathrm{d}$ can be related to the flight time $t$ between the foils via $t=d / v$, where $v$ is the velocity before the degrader. This velocity can be obtained from the velocity measured after the degrader with PRISMA and the energy loss of the ions in the degrader.

The spectra for the three $\mathrm{Cu}$ isotopes under study show no indication of transitions feeding any of the $7 / 2^{-}$states. Under the assumption that the feeding mechanism for these states is fast compared to their decay, the lifetime $\tau$ was 

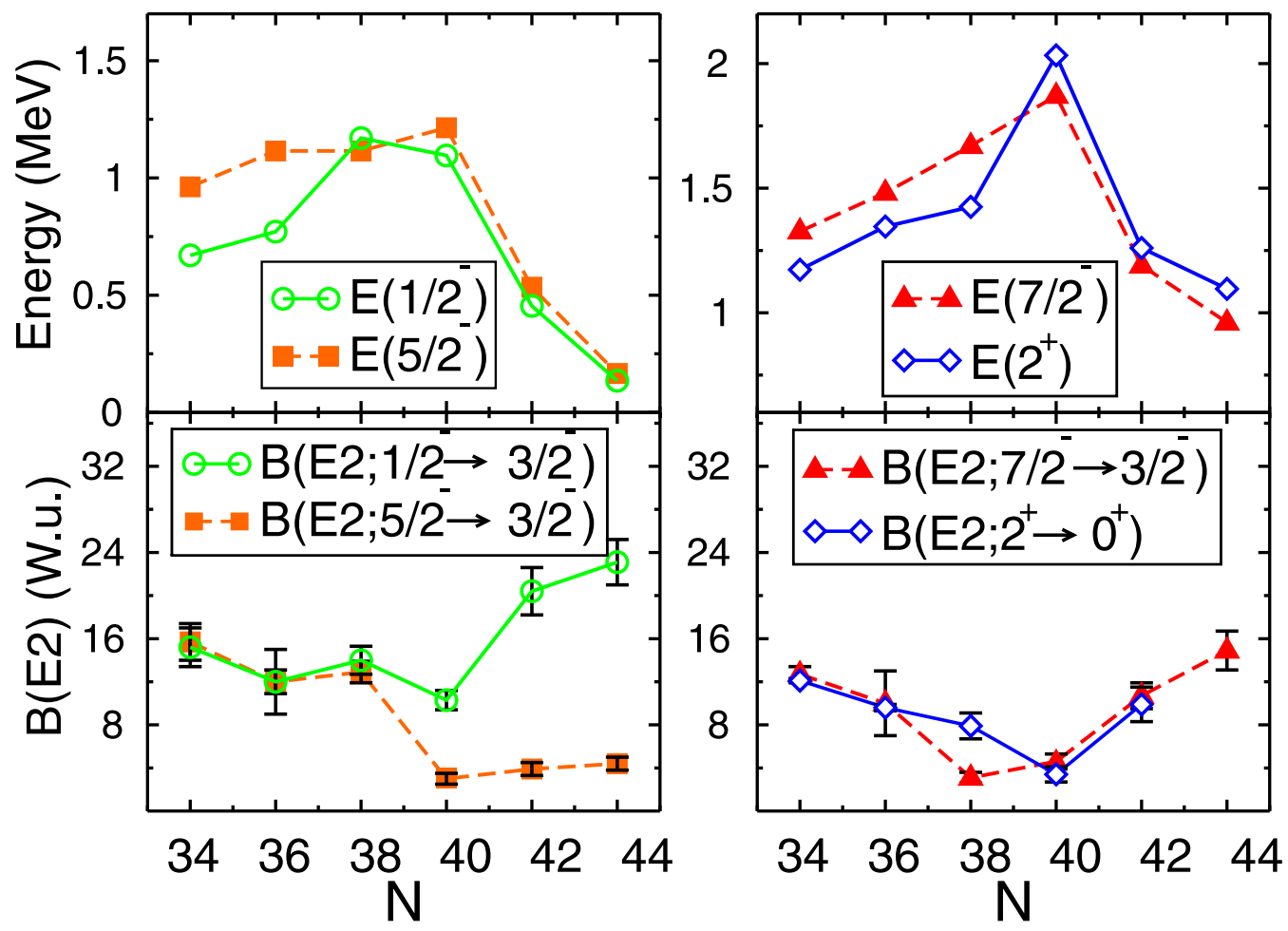

Fig. 34. Top panel: Systematics of the energies of the $1 / 2^{-}, 5 / 2^{-}$, and core-coupled $7 / 2^{-}$states in the ${ }^{63-73}$ Cu nuclei and the $2^{+}$levels in the even-even ${ }^{62-70} \mathrm{Ni}$ nuclei. Bottom panel: Experimental B(E2) values in the ${ }^{63-73} \mathrm{Cu}$ nuclei, and ${ }^{62-70} \mathrm{Ni}$ nuclei. The lines connecting the experimental points are to guide the eyes.

Source: Adapted from Ref. [160].

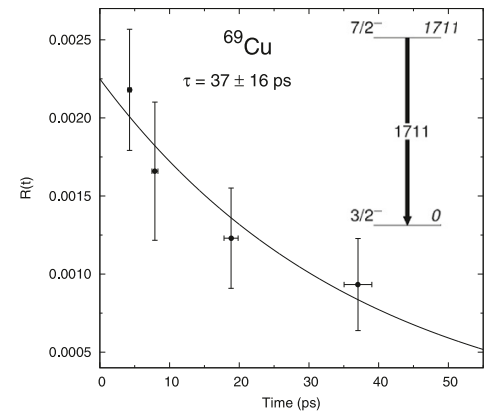

\begin{tabular}{ccccccc}
\hline$J_{f}^{\pi} \rightarrow J_{i}^{\pi}$ & $\begin{array}{c}E_{\text {level }}^{\text {exp }} \\
(\mathrm{keV})\end{array}$ & $\begin{array}{c}E_{\text {level }}^{\text {theor }} \\
(\mathrm{keV})\end{array}$ & $\begin{array}{c}E_{\gamma}^{\exp } \\
(\mathrm{keV})\end{array}$ & $\begin{array}{c}E_{\gamma}^{\text {theor }} \\
(\mathrm{keV})\end{array}$ & $\begin{array}{c}B(E 2)^{\exp } \\
\left(e^{2} \mathrm{fm}^{4}\right)\end{array}$ & $\begin{array}{c}B(E 2)^{\text {theor }} \\
\left(e^{2} \mathrm{fm}^{4}\right)\end{array}$ \\
\hline
\end{tabular}

\begin{tabular}{lccccccc}
\hline${ }^{69} \mathrm{Cu}$ & & & & & & & \\
$5 / 2_{1}^{-} \rightarrow 3 / 2_{1}^{-}$ & 1214 & 1249 & 1214 & 1249 & $50(8)$ & 28.5 \\
$7 / 2_{1}^{-} \rightarrow 3 / 2_{1}^{-}$ & 1711 & 2186 & 1711 & 2186 & $1.5(6)$ & 0.05 \\
$7 / 2_{2}^{-} \rightarrow 3 / 2_{1}^{-}$ & 1872 & 1862 & 1872 & 1862 & $77(12)$ & 46 \\
${ }^{71} \mathrm{Cu}$ & & & & & & & \\
$5 / 2_{1}^{-} \rightarrow 3 / 2_{1}^{-}$ & 534 & 276 & 534 & 276 & $68(8.8)$ & 54 \\
$7 / 2_{1}^{-} \rightarrow 3 / 2_{1}^{-}$ & 981 & 1426 & 981 & 1426 & $44(20)$ & 14.4 \\
$7 / 2_{2}^{-} \rightarrow 3 / 2_{1}^{-}$ & 1189 & 1115 & 1189 & 1115 & $187(2)$ & 179 \\
${ }^{73} \mathrm{Cu}$ & & & & & & \\
$5 / 2_{1}^{-} \rightarrow 3 / 2_{1}^{-}$ & 166 & 0 & 166 & -62 & $80(9)$ & 65 \\
$7 / 2_{1}^{-} \rightarrow 3 / 2_{1}^{-}$ & 961 & 937 & 961 & 875 & $270(3)$ & 251 \\
$7 / 2_{3}^{-} \rightarrow 5 / 2_{1}^{-}$ & 1298 & 1128 & 1132 & 1128 & $20(11)$ & 0.02 \\
$5 / 2_{2}^{-} \rightarrow 5 / 2_{1}^{-}$ & 1298 & 1203 & 1132 & 1203 & $20(11)$ & 11.4 \\
\hline \hline
\end{tabular}

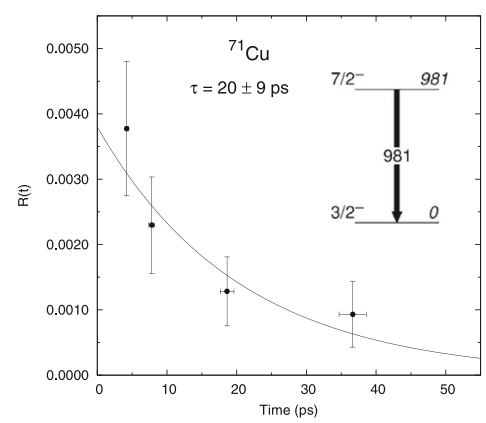

Fig. 35. Top-left and bottom panels: Experimental $\mathrm{R}(\mathrm{t})$ values(with $\mathrm{R}(\mathrm{t})$ defined in the text) as a function of the flight time between the target and degrader foils of the plunger device for the $7 / 2_{1}^{-} \rightarrow 3 / 2_{1}^{-}$state in ${ }^{69} \mathrm{Cu}$ (top) and in ${ }^{71} \mathrm{Cu}$ (bottom) and the result of the exponential fits. Right panel: table with experimental and predicted quantities for the ${ }^{69,71,73} \mathrm{Cu}$ isotopes. The measured values with the AGATA experiment at LNL are marked with rectangles.

Source: Adapted from Ref. [162]. 

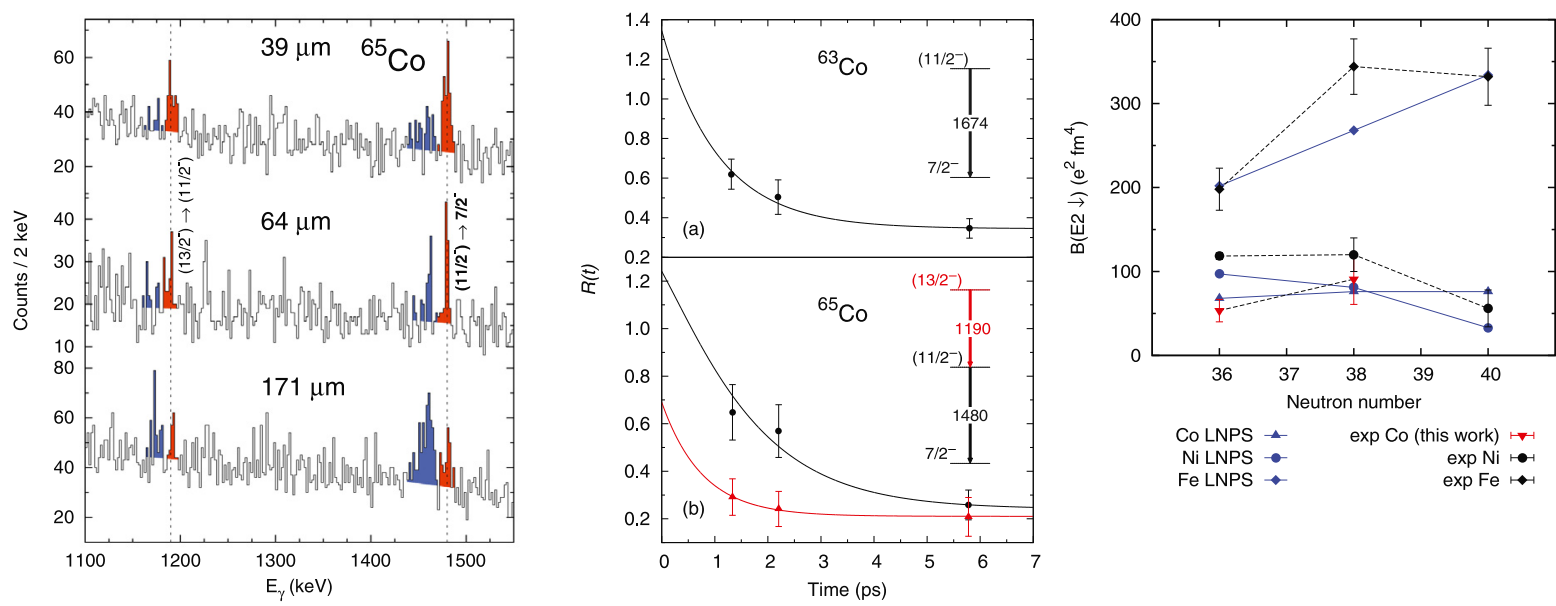

Fig. 36. Left panel: Portion of the spectra measured with AGATA including the transitions of interest. The three spectra correspond to different distances of the degrader foil of the plunger device. Central panels: Values of $\mathrm{R}(\mathrm{t})$, namely the ratio of intensity before and after the degrader of the plunger for the transitions $11 / 2^{-} \rightarrow 7 / 2^{-}$for ${ }^{63} \mathrm{Co}$ (top) and for ${ }^{65} \mathrm{Co}$ (bottom). Values of the feeding $\mathrm{M} 1$ transition $13 / 2^{-} \rightarrow 11 / 2^{-}$are marked in red. Right panel: The two deduced B(E2) values in comparison with data for Ni and Fe isotopes and with the corresponding shell-model predictions based on the LNPS interaction.

Source: Adapted from Ref. [163].

obtained from $\mathrm{R}(\mathrm{t})=\exp (-\mathrm{t} / \tau)$. The method was validated by reproducing several known lifetimes in other nuclides (e.g., ${ }^{72} \mathrm{Zn}$ and ${ }^{76} \mathrm{Ge}$ ).

A comparison of the transition probabilities with shell-model calculations shows that the different possible configurations lie close in energy and that the wave function of the lowest $7 / 2^{-}$state can be rather mixed (see [162]). The adopted model reproduces well the position and collective nature of the second $7 / 2^{-}$state in ${ }^{69,71,73} \mathrm{Cu}$ that were interpreted as one proton in the $2 p_{3 / 2}$ orbital coupled to the $2_{1}^{+} \mathrm{Ni}$ core. The lower lying $7 / 2^{-}$states in ${ }^{69,71} \mathrm{Cu}$ at 1711 and $981 \mathrm{keV}$, respectively, were found to have a significant contribution from the $\pi 1 \mathrm{f}_{5 / 2} \times 2_{1}^{+}$of the ${ }^{68,70} \mathrm{Ni}$ structure. However, the calculated excitation energies for these states are systematically too high. Only the $\left(7 / 2^{-}\right)$state at $1298 \mathrm{keV}$ in ${ }^{73} \mathrm{Cu}$ could potentially be explained as a $2 \mathrm{p}-1 \mathrm{~h}$ excitation across the proton shell gap. The absence of a low-lying proton-hole state at $\mathrm{N}=40$ and 42 in the present shell-model calculations is consistent with a reduction of the $\mathrm{Z}=28$ gap toward $\mathrm{N}=$ 50. However, the state at $1298 \mathrm{keV}$ in ${ }^{73} \mathrm{Cu}$ and its transition strength could also be explained as a second $5 / 2^{-}$state with reasonable agreement between the shell-model calculations and the experimental values. Further experimental and theoretical studies are needed to clarify the low-energy structure in Cu nuclei with increasing number of neutrons in the $v 1 g_{9 / 2}$ orbital.

Similar collectivity is expected when the coupling to the $2^{+}$states in Ni takes place with a hole instead of a particle. To find evidence for this it is necessary to measure lifetimes also for candidate states (to deduce the B(E2) matrix elements) for Co isotopes, having $Z=27$, namely one proton less than the closed shell $Z=28$. This investigation of the evolution of collectivity with neutron number was made with two separate AGATA experiments, one performed at LNL and the other at the GANIL laboratory.

The experiment performed at LNL with AGATA concerned the ${ }^{63} \mathrm{Co}$ and ${ }^{65} \mathrm{Co}$ nuclei [163]. The focus was on the B(E2) value to deduce the transition matrix element for the $11 / 2^{-}$excited state for which lifetimes were measured with a plunger device using the RDDS method. These nuclei were populated as products of a multi-nucleon transfer process following the collision of a ${ }^{64} \mathrm{Ni}$ beam at $460 \mathrm{MeV}$ with a ${ }^{238} \mathrm{U}$ target. The left panel of Fig. 36 shows the portion of spectra including the transitions of interest measured at different distances (and thus time) of the degrader foil through which the $\gamma$-emitting nucleus is passing. The corresponding values of $\mathrm{R}(\mathrm{t})$, namely the ratio of intensity before and after the foil, is presented in the central panel of the same figure.

The more recent experiment performed with AGATA at GANIL provided values of the $\mathrm{B}(\mathrm{E} 2)$ for the $11 / 2^{-} \rightarrow 7 / 2^{-}$ transition in ${ }^{61,63} \mathrm{Co}$ [164]. These nuclei were populated in multi-nucleon transfer reactions from a $6.5 \mathrm{AMeV}{ }^{238} \mathrm{U}$ beam impinging on a ${ }^{64} \mathrm{Ni}$ target. The VAMOS++ spectrometer was used to detect and identify the target-like transfer products. It was positioned at $45 \mathrm{deg}$, close to the grazing angle of the reaction. A sample of the spectra used to extract the lifetime of the $11 / 2^{-} \rightarrow 7 / 2^{-}$transition corresponding to different distances $\mathrm{d}$ (in $\mu \mathrm{m}$ ) is shown in Fig. 37 . The colored areas were used for determining the decay curves. The area to the left is the shifted component while the right area is the unshifted component.

The deduced $\mathrm{B}(\mathrm{E} 2)$ values for the $(11 / 2)^{-} \rightarrow 7 / 2^{-}$transitions in the ${ }^{63,65} \mathrm{Co}$ are shown in the right panel of Fig. 36 together with the corresponding $\mathrm{B}\left(\mathrm{E} 2 ; 2^{+} \rightarrow 0^{+}\right)$values measured for the $\mathrm{Ni}$ and Fe isotones. One can observe that the $\mathrm{B}(\mathrm{E} 2)$ values for the $\mathrm{Ni} 2^{+} \rightarrow 0^{+}$transitions have a minimum at $\mathrm{N}=40$, which is consistent with the presence of a 

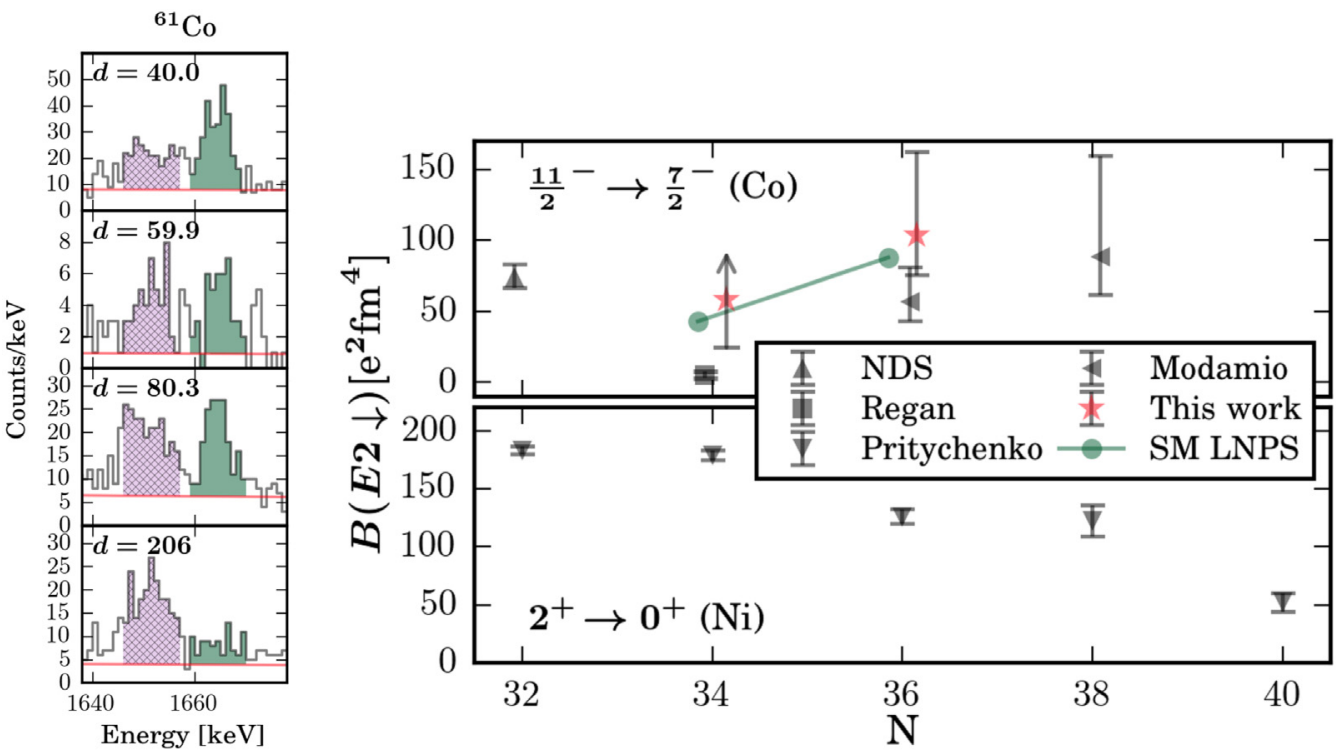

Fig. 37. Left panel: Four portions of the spectra used to extract the lifetime of the $11 / 2^{-} \rightarrow 7 / 2^{-}$transition in ${ }^{61}$ Co corresponding to different distances $\mathrm{d}$ (in $\mu \mathrm{m}$ ). The area to the left is the shifted component while the right area is the unshifted component. Right panel: The B(E2) values as a function of $\mathrm{N}$ where the stars are from the measurement with AGATA at GANIL and the horizontal triangles from the measurement with AGATA at LNL. The data for the Ni isotones shown in the bottom-right panel are for the $2^{+} \rightarrow 0^{+}$transitions. The green line shows the shell-model predictions with the LNPS interaction.

Source: Adapted from Ref. [164].

subshell gap while the systematics of the $\mathrm{B}(\mathrm{E} 2)$ values for the $2^{+} \rightarrow 0^{+}$transitions in Fe reveals an increasing collectivity towards $\mathrm{N}=40$. This is due to the fact that $\mathrm{Fe}$ as well as the neutron-rich $\mathrm{Cr}$ isotopes lie in the so-called third island of inversion [100]. The $\mathrm{B}(\mathrm{E} 2)$ of the two ${ }^{63,65} \mathrm{Co}$ isotopes is found to follow the same trend as their corresponding Ni isotones. This behavior strengthens the interpretation of these states as being of $\pi\left(\mathrm{f}_{7 / 2} \times 2^{+}\right)$Ni configuration.

$\mathrm{B}(\mathrm{E} 2)$ values were calculated employing the shell-model code ANTOINE, and using the LNPS [109] interaction. The used model space was the $p f$ shell for protons and the $0 \mathrm{f}_{5 / 2}, 1 \mathrm{p}_{3 / 2}, 1 \mathrm{p}_{1 / 2}, 0 \mathrm{~g}_{9 / 2}$, and $1 \mathrm{~d}_{5 / 2}$ orbits for neutrons. It was already reported in Ref. [109] that the inclusion of the neutron $1 d_{5 / 2}$ orbital is essential to reproduce the large quadrupole collectivity in this mass region. This can be understood in terms of the quasi-SU(3) approximate symmetry as was explained by the values of the neutron occupation numbers of the $g_{9 / 2}$ and the $d_{5 / 2}$ orbitals for the $11 / 2^{-}$excited states in ${ }^{63,65} \mathrm{Co}$ and the $2^{+}$states in $\mathrm{Ni}$ and Fe. It is found that the inclusion of these orbitals into the valence space leads to an increase of the deformation in Fe isotopes. The quadrupole correlations together with the monopole shifts of the single-particle energies of these orbitals, give rise to the large $\mathrm{B}(\mathrm{E} 2)$ values in Fe isotopes.

In the Co isotopes, the trend of the occupation of both orbitals follows quite closely the Ni isotopes except for the case of $\mathrm{N}=40$, in which the relative increase in the $\mathrm{d}_{5 / 2}$ occupation in ${ }^{67} \mathrm{Co}$ with respect to its isotone ${ }^{68} \mathrm{Ni}$ gives rise to a slightly larger $\mathrm{B}(\mathrm{E} 2)$ value. While the $\mathrm{B}(\mathrm{E} 2)$ values for Fe isotopes increase with $\mathrm{N}$ and those of Ni decrease at $\mathrm{N}=40$, the $\mathrm{B}(\mathrm{E} 2)$ values for the Co isotopes show a presumably constant trend. The calculations predict a larger $\mathrm{B}(\mathrm{E} 2)$ value in ${ }^{67} \mathrm{Co}$ than that of ${ }^{68} \mathrm{Ni}$. However, further experimental studies are needed for ${ }^{67} \mathrm{Co}$ in order to validate such theoretical predictions.

To get a better picture of the coupling of single particles with the excitation of a $\mathrm{Ni}$ core, it is interesting to recall here additional data. As reported in $[165,166]$ and $[167]$ and the $9 / 2^{+}$states was investigated in a chain of $\mathrm{Cu}$ isotopes to search for couplings to the octupole vibration of the Ni core. In particular, lifetimes for the nuclei ${ }^{61,65,67} \mathrm{Cu}$ and the corresponding $\mathrm{B}(\mathrm{E} 3)$ were interpreted as due to the coupling of the ground-state proton in $\mathrm{p}_{3 / 2}$ orbital to the $3^{-}$phonon of the Ni core $(Z=28)$. The levels involved in these couplings are shown in the left panels of Fig. 38.

The measured $\mathrm{B}(\mathrm{E} 3)$ values for the $9 / 2^{+} \rightarrow 3 / 2^{-}$transitions in ${ }^{61,65,67} \mathrm{Cu}$, as deduced from lifetimes measurements using both the plunger device and the fast timing techniques, are shown in right panel of Fig. 38. For these data transfer reactions below and around the Coulomb barrier were employed [165] and their interpretation is shown with the curves in the right panel of Fig. 38. This interpretation is based on an extension of the weak-coupling particle-phonon model to open-shell nuclei that is described in Ref. [168]. For the ${ }^{64,66} \mathrm{Ni}$ nuclei core microscopic HF-BCS plus QRPA calculations were performed, providing single-particle and quasi-particle states and giving evidence that neutrons are superfluid while protons are not. The phonon states were also calculated and in particular the collective $3^{-}$states of interest (see [166] and references therein). Two different microscopic Skyrme parameter sets were employed (SkX and Sly5). For neutrons, a zerorange, density-dependent surface pairing interaction was used, and its parameters were fitted to reasonably reproduce the pairing gaps along the $\mathrm{Ni}$ isotopic chain in the region. 


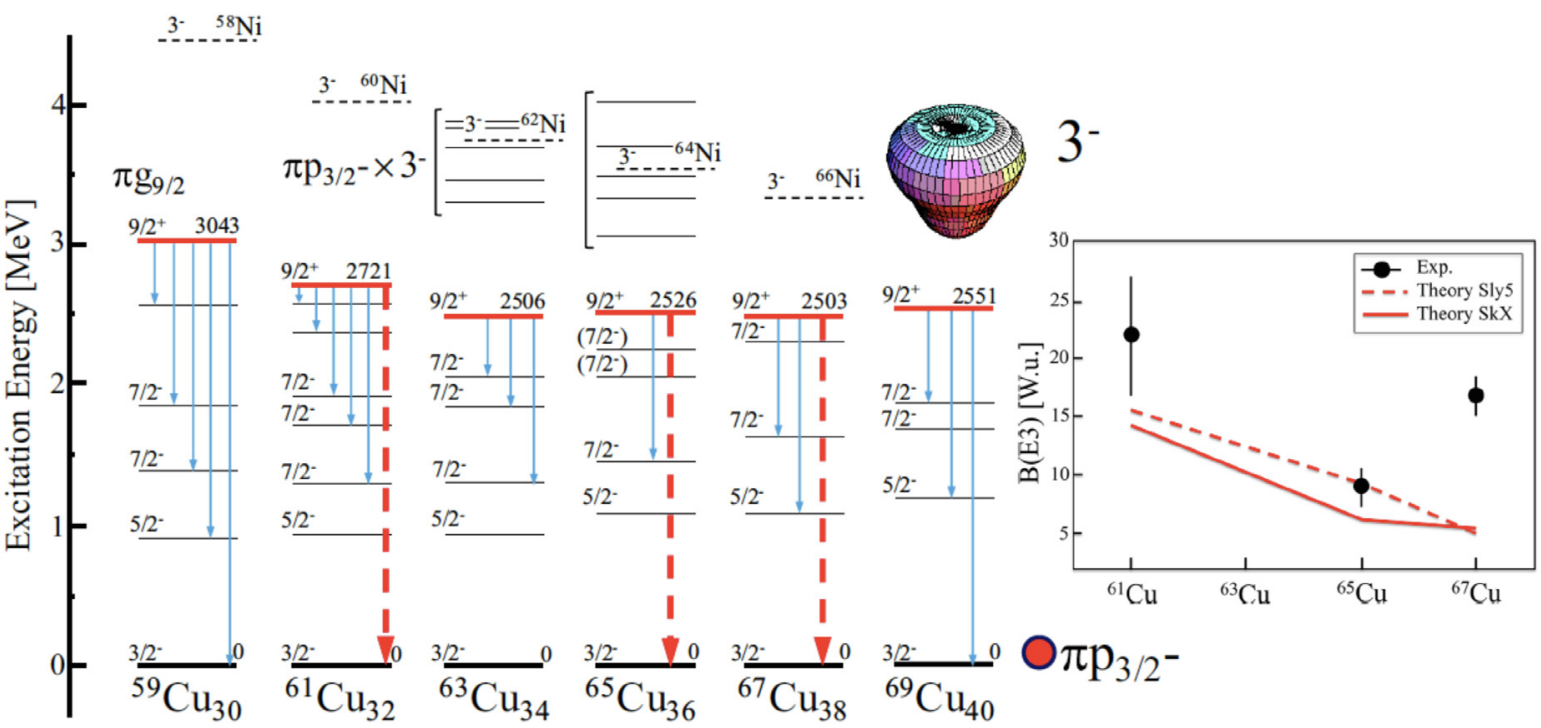

Fig. 38. Left panel: Energy levels and $\gamma$-ray branchings for the first excited $9 / 2^{+}$states in ${ }^{59-69} \mathrm{Cu}$ odd-even isotopes. The $3^{-}$octupole states in the corresponding $\mathrm{Ni}$ isotopes for which the $\mathrm{B}(\mathrm{E} 3)$ was measured are indicated by dashed lines. In the case of ${ }^{61,65,67} \mathrm{Cu}$, the lifetimes of the $9 / 2^{+}$ states (candidate for being core-excitation states, $\pi \mathrm{p}_{3 / 2} \times \mathrm{Ni}\left(3^{-}\right.$coupled states) were measured [165]. Right panel: $\mathrm{B}$ (E3) data for the neutron-rich odd-even $\mathrm{Ni}$ isotopes in comparison with predictions. The calculations were performed within the schematic Bohr and Mottelson particle-phonon coupling model, for the $9 / 2^{+}$states in ${ }^{61,65,67} \mathrm{Cu}$ isotopes.

Source: Adapted from Ref. [166], with kind permission of EPJ.

In ${ }^{65,67} \mathrm{Cu}$ the energy of the $9 / 2^{+}$state is found to be $400-500 \mathrm{keV}$ higher than in the experiment. In ${ }^{61} \mathrm{Cu}$ the agreement is better than $200 \mathrm{keV}$ but for this nucleus the experimental value of the energy of the $3^{-}$was used. This suggests that the wave function of the $9 / 2^{+}$state has a dominant component from the low-lying octupole phonon. However, the comparison between data and calculations of the quantity B(E3), shown in the right panel of Fig. 38, clearly points to a more complex structure. The simple picture of a single proton coupled to the octupole phonon in the corresponding Ni core does not seem to give a good account for the data of ${ }^{61} \mathrm{Cu}$ and ${ }^{67} \mathrm{Cu}$. Consequently for the $9 / 2^{+}$states contributions from other types of excitations could play a significant role. Results from recent spectroscopy works in the neutron-rich Ni region support this interpretation and also point to a rather complex scenario of coexistence of spherical, oblate and prolate shapes, in the excitation energy region below $4 \mathrm{MeV}$. The need for a more refined theoretical description is thus clear. Other interesting recent studies on the search of states with particle-phonon coupling structure in other mass regions are reported in [166].

\subsubsection{Octupole vibration coupling in ${ }^{207} \mathrm{~Pb}$}

The coupling of hole neutron states in ${ }^{207} \mathrm{~Pb}$ with the excitations of the ${ }^{208} \mathrm{~Pb}$ core is particularly interesting since ${ }^{208} \mathrm{~Pb}$ has the uncommon feature of having as a first excited state an octupole vibration of very collective nature with a reduced transition probability $B\left(E 3,3^{-} \rightarrow 0^{+}\right)=34.0(5)$ W.u. This $3^{-}$state is interpreted as a one-phonon excitation corresponding to a nuclear surface vibration of octupole character while its microscopic structure is understood as the coherent and collective superposition of one-particle-one-hole (1p-1h) excitations across the neutron and proton shell gaps.

The identification of the members of the two-phonon octupole quartet has motivated several efforts. Candidates for the lower-spin members have been proposed ([169] and references therein) but the $6^{+}$member has not been identified yet. The presence of fragmentation for the $0^{+}, 2^{+}$, and $4^{+}$states decaying via weak E1 and E2 transitions was proposed, lacking a clear evidence for a double-octupole nature.

The octupole excitations can couple either weakly or strongly to single-particle states in neighboring nuclei with one valence particle or hole. The particle-octupole-phonon model favors strong coupling between the orbitals $j_{1}=l_{1} \pm 1 / 2$ and $j_{2}=l_{2} \pm 1 / 2$ if $\left|j_{1}-j_{2}\right|=\left|l_{1}-l_{2}\right|=3$, preserving the relative orientation of the spin and orbital angular momenta (see [168],(Vol. II, p.419)). Because of the strong coupling, such states are expected to mix.

The octupole vibration coupling to the low-spin ground state in ${ }^{207} \mathrm{~Pb}$ have been investigated earlier [170,171]. The $5 / 2^{+}$state at $2624 \mathrm{keV}$ and $7 / 2^{+}$state at $2662 \mathrm{keV}$ have been interpreted as members of the multiplet obtained by the phonon weak coupling with the low-spin $v \mathrm{p}_{1 / 2}^{-}$state. The corresponding reduced transition probabilities were found to be $B\left(E 3,5 / 2^{+} \rightarrow 1 / 2^{-}\right)=30(3)$ W.u. and $B\left(E 3,7 / 2^{+} \rightarrow 1 / 2^{-}\right)=28(2)$ W.u. The small positive energy shifts, $+9 \mathrm{keV}$ and $+47 \mathrm{keV}$ relative to the octupole phonon, could be related to the Pauli blocking of the $v p_{1 / 2}$ orbital.

Among the available neutron orbitals of ${ }^{207} \mathrm{~Pb}$, only the $\mathrm{j}_{1}=v i_{13 / 2}$ and $\mathrm{j}_{2}=v f_{7 / 2}$ satisfy the strong coupling rule. The corresponding states, $13 / 2^{+}$and $7 / 2^{-}$, are well studied and found to have a dominant single-particle character. At variance, 

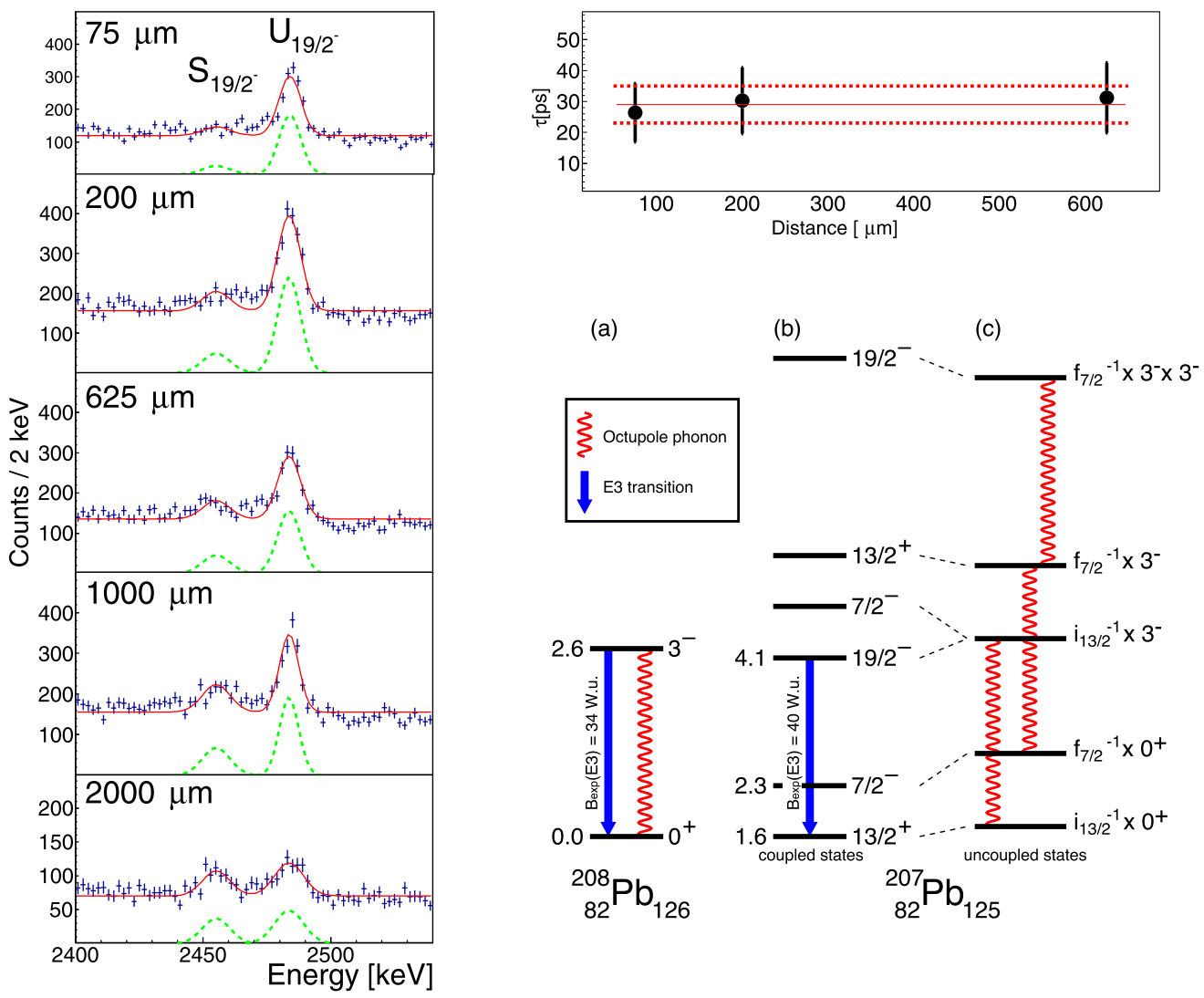

Fig. 39. Left panels: $\gamma$-ray spectra for different distances of the degrader foil of the plunger that were used to extract the lifetime of the $19 / 2^{-}$state from the $19 / 2^{-} \rightarrow 13 / 2^{+}$transition in ${ }^{207} \mathrm{~Pb}$. Right top panel: the measured lifetime in ps. Right bottom panel: schematic illustration of the strong coupling multi phonon model. (a) Octupole phonon in ${ }^{208} \mathrm{~Pb}$. (b) Experimental partial level scheme of ${ }^{207} \mathrm{~Pb}$ showing its strong E3 transition. (c) Proposed octupole phonon coupling in ${ }^{207} \mathrm{~Pb}$ involving a single octupole phonon excitation based on the $\mathrm{i}_{13 / 2}$ orbital and a double octupole phonon excitation based on the $\mathrm{f}_{7 / 2}$ orbital.

Source: Adapted from Ref. [169].

the $2485 \mathrm{keV}$ transition from the decay of the $19 / 2^{-}$state to the $13 / 2^{+}$state, was found to have an E3 character [172]. The $13 / 2^{+}, 7 / 2^{-}$, and $19 / 2^{-}$states were analyzed in terms of particle-octupole-vibration coupling in [173] using the experimentally known energies and assuming the dominance of the above-mentioned orbitals. This coupling scheme is illustrated in Fig. 39. The $\mathrm{B}\left(\mathrm{E} 3,19 / 2^{-} \rightarrow 13 / 2^{+}\right)$value was predicted to be enhanced as compared to $B\left(E 3,3^{-} \rightarrow 0^{+}\right)$in ${ }^{208} \mathrm{~Pb}$, due to the strong mixing to the double-octupole-phonon strengths.

A measurement at GANIL with AGATA was devoted to obtain the experimental $\mathrm{B}\left(\mathrm{E} 3,19 / 2^{-} \rightarrow 13 / 2^{+}\right)$to test the predicted enhancement. The experimental finding deduced from the lifetime measurement corresponds to a large value of the reduced transition probability, namely $B\left(E 3,19 / 2^{-} \rightarrow 13 / 2^{+}\right)=40(8)$ W.u. Such very collective character indicates that the dominant component of this state is a single-hole excitation coupled to the octupole phonon of the ${ }^{208} \mathrm{~Pb}$ core. The lower energy of the $2485 \mathrm{keV}$ transition in ${ }^{207} \mathrm{~Pb}$, as compared to the $2615 \mathrm{keV}$ transition in ${ }^{208} \mathrm{~Pb}$, is consistent with a mixing with a state containing the double octupole-phonon excitation. The measured reduced transition probability is compatible with a contribution from the two-to-one-octupole-phonon E3 transition. Further information on the doubleoctupole-phonon state can be obtained by a more precise lifetime measurement of the $19 / 2^{-}$state in ${ }^{207} \mathrm{~Pb}$ or of the corresponding $21 / 2^{+}$state in ${ }^{209} \mathrm{~Pb}$, where the $\mathrm{B}(\mathrm{E} 3)$ was predicted to be 50 W.u. [173]. In addition, a more accurate measurement of the lifetime of the $15 / 2^{-}$state in ${ }^{209} \mathrm{~Pb}$ is mandatory to improve the precision of the $\mathrm{E} 3$ effective charge.

\subsubsection{Evolution of the $B(E 2)$ for $4^{+}$states in Fe isotopes}

Data for the $\mathrm{B}(\mathrm{E} 2)$ of the $4^{+}$state in ${ }^{62,64} \mathrm{Fe}$ isotopes were obtained with the same AGATA experiment at GANIL providing data for ${ }^{61,63} \mathrm{Co}$, namely with the multi-nucleon transfer reactions from a $6.5 \mathrm{AMeV}{ }^{238} \mathrm{U}$ beam on a ${ }^{64} \mathrm{Ni}$ target.

Some of the spectra used to extract the lifetime of the $4^{+}$state in ${ }^{62,64} \mathrm{Fe}$ from the $4^{+} \rightarrow 2^{+}$transition are shown in the left panels of Fig. 40. Each panel corresponds to different distances $\mathrm{d}$ (in $\mu \mathrm{m}$ ) of the plunger foil. The area to the left is the shifted component while the right area is the unshifted component. A clear shift of the two components is visible. Lifetimes of the $4^{+}$states were deduced after correction for feeding. 

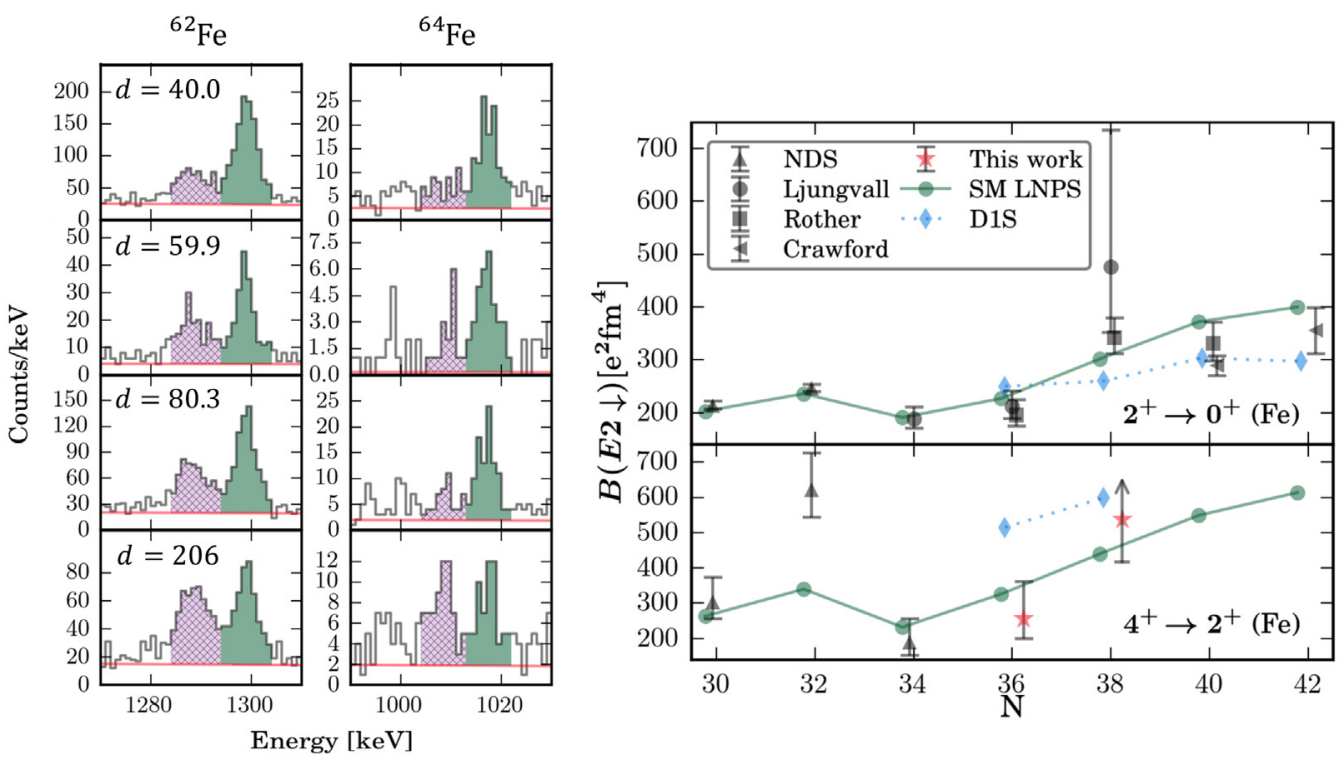

Fig. 40. Left panels: Portions of the spectra used to extract the lifetime of $4^{+}$state from the $4^{+} \rightarrow 2^{+}$transition in ${ }^{62,64}$ Fe corresponding to different distances $\mathrm{d}$ (in $\mu \mathrm{m}$ ). The area to the left is the shifted component while the right area is the unshifted component. Right panels: (top) the measured $\mathrm{B}$ (E2) values for the even Fe isotopes as a function of the neutron number $\mathrm{N}$ for the $2^{+} \rightarrow 0^{+}$transitions and (bottom) the $\mathrm{B}$ (E2) values for the $4^{+} \rightarrow 2^{+}$transitions. The green lines show the shell-model predictions with the LNPS interaction and the blue lines are from the DIS model. Source: Adapted from Ref. [164].

In the right panel of Fig. 40 experimental and predicted values for the reduced transition strengths in the iron isotopes ${ }^{56,68} \mathrm{Fe}$ are shown for the $2^{+} \rightarrow 0^{+}$and $4_{1}^{+} \rightarrow 2_{1}^{+}$transitions. A small increase is observed by going from $\mathrm{N}=34$ to $\mathrm{N}=$ 36. These iron isotopes have $R_{42}=E\left(4^{+}\right) / E\left(2^{+}\right)$ratios between the harmonic vibrator limit $\left(R_{42}=2\right)$ and the rotational limit $\left(R_{42}=3.3\right)$ and the additional information on $\mathrm{B}\left(\mathrm{E} 2 ; 4_{1}^{+} \rightarrow 2_{1}^{+}\right) / \mathrm{B}\left(\mathrm{E} 2 ; 2_{1}^{+} \rightarrow 0^{+}\right)$ratios (smaller than 2$)$ indicates the presence of a soft rotor structure.

Large-scale shell-model calculations using the modified LNPS interaction [109], also shown in the right panel of Fig. 40, describe the data within the experimental errors. The increase in collectivity can be explained by a decrease of the subshell gap at $\mathrm{N}=40$, driven by the monopole proton-neutron part of the effective interaction, favoring neutron excitations into the quasi-SU(3) partners $v 1 g_{9 / 2}-v 2 d_{5 / 2}$. For the $4_{1}^{+}$state the occupation number for the $v 1 g_{9 / 2}$ orbital increases from 1.14 in ${ }^{62} \mathrm{Fe}$ to 2.13 in ${ }^{64} \mathrm{Fe}$. More details for these calculations are given in [164].

Calculations of the type beyond-mean-field CHFB $+5 \mathrm{DCH}$ using the D1S interaction were also compared with the experimental data (see [164] for details). Although the overall trend of increasing collectivity with increasing neutron number for the iron isotopes is clearly seen, the beyond-mean-field calculations suggest a vibrational-like structure and no clear change in structure close to $\mathrm{N}=38$. This might originate from the lack of an explicit tensor term in the force.

\subsection{The low energy tail of the E1 and E2 response}

The accumulation of strength in the tail of the GDR, known as Pygmy Dipole Resonance (PDR), is attracting particular interest because this strength contributes rather significantly to the nuclear polarizability and because it plays an important role in the competition with neutron capture in the r-process nucleosynthesis. The polarizability gives information on the neutron matter and thus it has some implications on the modeling of neutron stars. These issues are discussed in detail in the two recent review papers [174,175].

Several models ([175] and reference therein) describe the PDR states as excitations of excess neutron or proton matter (depending on the isospin asymmetry of the nucleus) at the nuclear-surface layer corresponding to a vibrational motion of the nuclear skin against the core. In some models the total PDR strength was found to have a role in the determination of the neutron skin thickness.

One important feature of the pygmy states, as deduced in different theoretical models, is in the shape of the transition densities. The PDR states in neutron-rich nuclei exhibit always the same peculiar features: proton and neutron oscillate in phase inside the nucleus while in the surface region only the neutron part survives. This fact can be used as a theoretical definition of the states associated to the PDR. Since the PDR states are associated to surface oscillations it is interesting to study them with probes interacting mainly at the surface. This was the case of experiments using the inelastic scattering of alpha beams and measuring the gamma decay [176].

In order to get more insight on the nature of the pygmy states it is important to use different probes to excite this states and compare the different results. This has motivated a series of experiments performed at LNL-INFN with the 

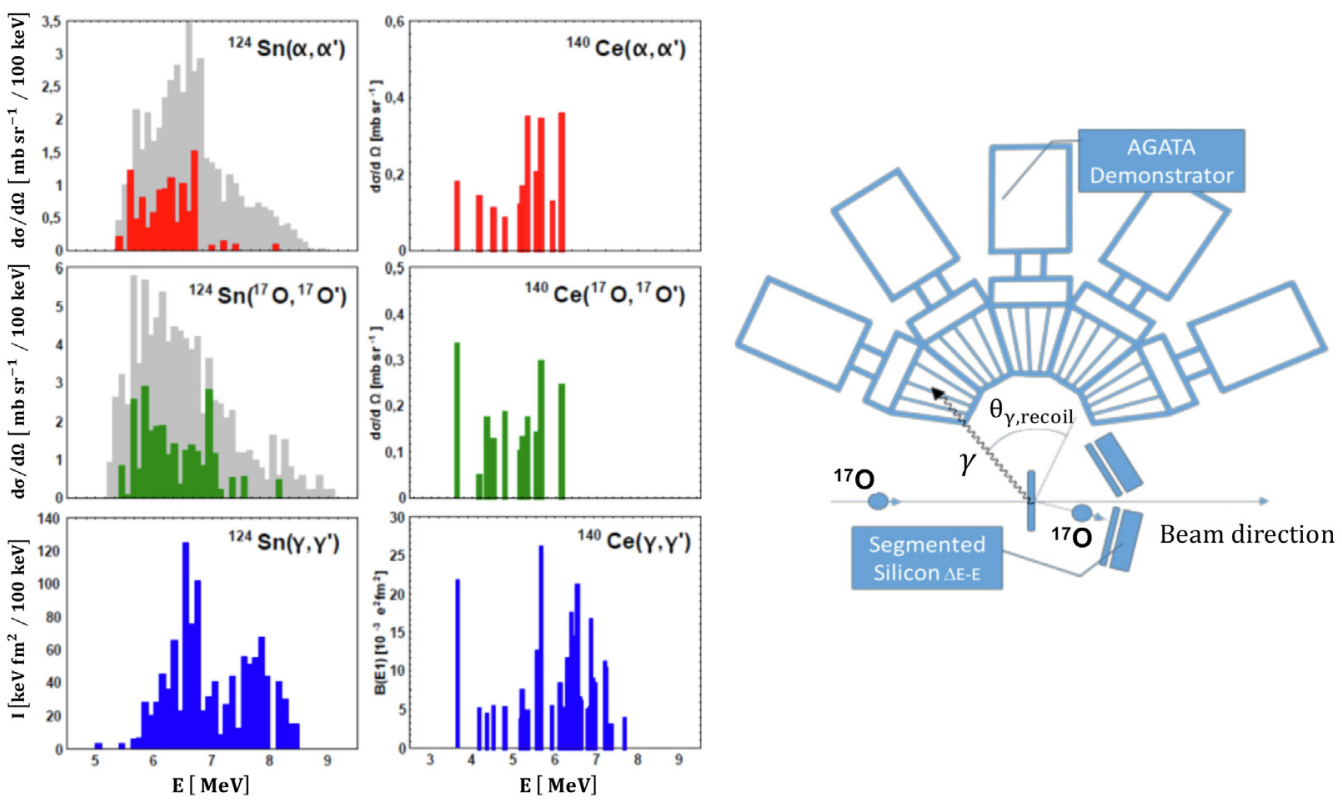

Fig. 41. Left and middle panels: The measured strengths of $\mathrm{E} 1$ type are shown for the nuclei ${ }^{124} \mathrm{Sn}$ and ${ }^{140} \mathrm{Ce}$, respectively. The data in the central-left and central-middle panels were obtained using the reaction $\left({ }^{17} \mathrm{O},{ }^{17} \mathrm{O} \gamma\right)$ [178] and [181]. The data in the top-left and top-middle panels are from $(\alpha$, $\left.\alpha^{\prime} \gamma\right)[182,183]$ and those in the left- and middle-bottom panels are from the $\left(\gamma, \gamma^{\prime}\right)$ reactions [184,185] and [186]. Rightmost panel: The schematic set up of the AGATA experiment for measuring the $\left({ }^{17} \mathrm{O},{ }^{17} \mathrm{O}^{\prime} \gamma\right)$ reaction is shown.

Source: Adapted from Ref. [180] and [36]. With kind permission of EPJ.

AGATA spectrometer using inelastic scattering of ${ }^{17} \mathrm{O}$ ions at $340 \mathrm{MeV}$ [177-181]. In those experiments the scattered ${ }^{17} \mathrm{O}$ ions were detected with two segmented $\Delta \mathrm{E}$-E silicon telescopes (pixel type, positioned at forward angles of $12-25^{0}$ ). The set up is shown in a schematic way in the right part of Fig. 41.

Some results for the measured cross sections for the ${ }^{124} \mathrm{Sn}\left({ }^{17} \mathrm{O},{ }^{17} \mathrm{O} \gamma\right)[178]$ and ${ }^{140} \mathrm{Ce}\left({ }^{17} \mathrm{O},{ }^{17} \mathrm{O} \gamma\right)$ [181] reactions are shown in the left part of Fig. 41. For comparison, the cross sections measured in $\alpha$-scattering experiments for the same nuclei [182,183] (top panels), and the strengths measured in photon-scattering experiments (bottom panels) [184-186] are also shown. One clearly sees (for both the ${ }^{124} \mathrm{Sn}$ and ${ }^{140} \mathrm{Ce}$ nuclei) that the PDR states split in two regions, one at lower energy populated by the nuclear probes of isoscalar type and the one at higher energy populated by the Coulomb interaction of isovector type.

In addition, the analysis of the cross-section data using the DWBA approach indicates that the states populated via isoscalar probes have a transition density typical of the pygmy states. This is shown in Fig. 42, where the top part gives the values of the isoscalar strength deduced from all the measurements performed with AGATA at LNL. The bottom left part of Fig. 42 shows an example on how the analyses to extract the isoscalar energy weighted sum rule (ISEWSR) were made. There data (circle) and predictions (lines) within the DWBA approach are shown for $1^{-}$states in the energy region 5.5-7 MeV in the ${ }^{124} \mathrm{Sn}$ nucleus. The red curve was obtained using the form factor shown in the right panel which was derived by folding the transition density (isoscalar) shown in the inset. The calculations in the left panel displayed with the green and black curves are obtained using the Coulomb (C) and isovector (IV) form factors, respectively [178]. The deduced ISEWSR comes from a comparison between data and calculations. The found values of the isoscalar dipole strength at low energy are rather small and consistent in some cases with theoretical predictions. More work is being made also using proton beams to excite pygmy states and to get better insight into the structure of $1^{-}$states at around the neutron binding energies.

\subsubsection{The E1 strength far from stability}

The investigation of the E1 strength in nuclei far from stability is a subject of particular attention. The resulting picture deduced so far from the few existing experiments comprises mainly two types of mechanisms associated to the nuclear structure. The first mechanism manifests itself in light nuclei and the second primarily in medium-heavy nuclei which are neutron rich. In light nuclei with halo structures in the wave function, specific transitions among single-particle states have enhanced E1 strength, while neutron-rich nuclei with medium mass number A have enhanced E1 strength at around the neutron binding energy, interpreted as due to collective effects connected to the neutron skin.

With the AGATA array an experiment was made concentrating on the search of E1 strength in two neutron-rich Fe isotopes to deduce the possible presence of neutron-skin effects. Instead, an experiment made with the array GRETINA 


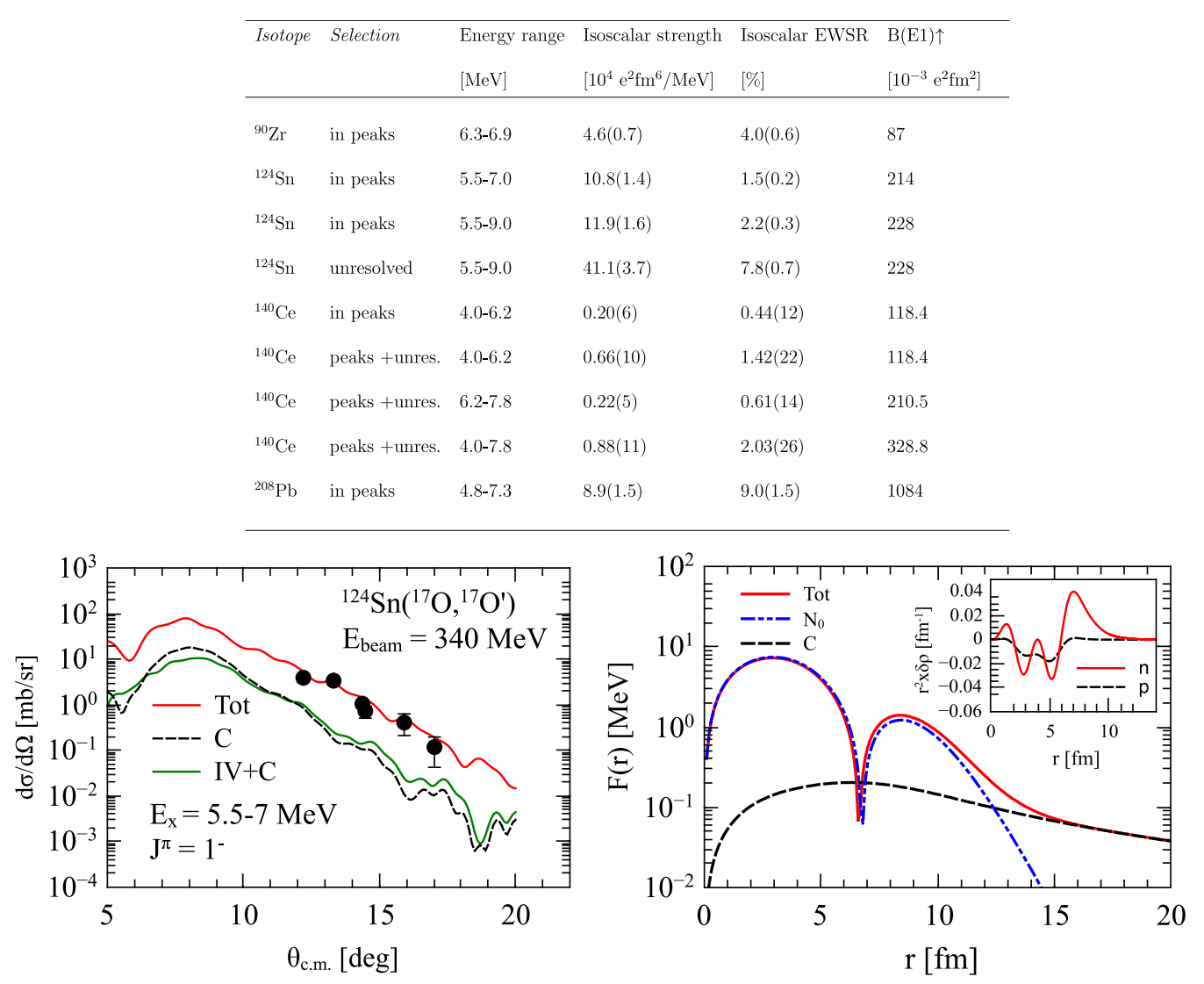

Fig. 42. Top panel: A table is given reporting the $\mathrm{B}(\mathrm{E} 1)$ values and the isoscalar strength deduced from all the measurements with the reaction $\left({ }^{17} \mathrm{O}\right.$, ${ }^{17} \mathrm{O}^{\prime} \gamma$ ) performed with AGATA at LNL. bottom left panel: Data (circles) and predictions (lines) for $1^{-}$states in the energy region $5.5-7$ MeV for the ${ }^{124} \mathrm{Sn}$ nucleus. bottom right panel: The red curve was obtained using a form factor which was derived by folding the transition density (isoscalar) shown in the inset. The calculations in the left panel, displayed with the green and black curves, are obtained using the Coulomb (C) and isovector (IV) form factors, respectively.

Source: Adapted from Ref. [178].

focused on E1 transitions in weakly bound nuclei, by measuring the $\gamma$-decay in the ${ }^{27} \mathrm{Ne}$ nucleus. In the nuclear chart this nucleus is located next to the dripline and also in the vicinity of the island of inversion.

The main conclusions from these two experiments are briefly discussed also in view of the need of future new experimental and theoretical investigations.

\subsubsection{Single-particle structure of E1 transitions in weakly bound nuclei via $\gamma$ spectroscopy}

Unusual structures denoted as nuclear halos are ascribed to the spatially extended wave function of valence nucleons and their special feature is to be accompanied by the enhanced low-energy electric dipole (E1) strength. In light p- and sd-shell nuclei close to the neutron drip line, the significant $2 s_{1 / 2}$-wave strength in the ground-state wave function was recognized to be crucial in halo formation. The resultant strong E1 transitions among associated discrete states were also observed and interpreted in terms of the excitation of the valence nucleon weakly coupled to a core state. It has to be pointed out that the strong low-energy E1 mode requires the appearance of two particular conditions. One is a modification of the shell structure implying that a non-normal parity state can intrude at low excitation energy and the second is that the valence neutron has a small separation energy, namely less than $1 \mathrm{MeV}$ and it occupies a state with low angular momentum (with $\mathrm{l}=0,1$ ), and this allows the extended wave function to enhance the E1 strength [187]. Consequently, when an enhancement of the low-energy E1 strength is observed one can interpret it as a sign for a structural change near the drip line.

The experiment made with GRETINA on the ${ }^{27}$ Ne nucleus [187] has measured the lifetimes from line shape and plunger experiment of transitions from states whose structure involves the spd orbitals (see the level scheme in the leftmost panel of Fig. 43). More specifically the orbitals of interest are indicated in rightmost panel Fig. 43. It is found that E1 transition $1 / 2^{+} \rightarrow 3 / 2^{-}$in ${ }^{27} \mathrm{Ne}$ has the strongest strength observed among the bound discrete states in this mass region, namely the $\mathrm{B}(\mathrm{E} 1)$ has a lower bound of $0.030 \mathrm{e}^{2} \mathrm{fm}^{2}$ or $0.052 \mathrm{~W}$.u. This value is at least 30 times larger than that measured for the $3 / 2^{-}$ decay to the $3 / 2^{+}$ground state $\left(6.7_{-1.3}^{+2.1} 10^{-4} \mathrm{e}^{2} \mathrm{fm}^{2}\right)$. A comparison of this result with large-scale shell-model calculations 

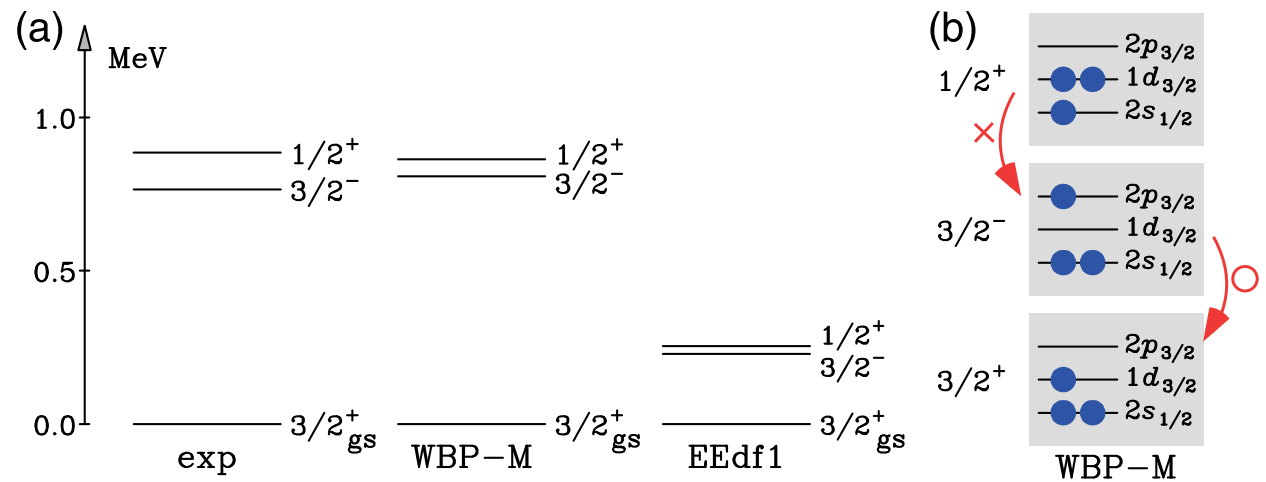

Fig. 43. Panel (a): The level scheme of ${ }^{27} \mathrm{Ne}$ is compared to the WBP-M and EEdf1 shell-model calculations. The dominant configurations given by the WBP-M calculations are illustrated for three valence neutrons outside the fully occupied $1 \mathrm{~d}_{5 / 2}$ orbital. Panel (b): The diagrams with the arrows indicate the allowed (circle) or forbidden (cross) E1 transitions among the states. The WBP-M calculation gives $\mathrm{B}\left(\mathrm{E} 1 ; 1 / 2^{+} \rightarrow 3 / 2^{-}\right)=7.5 \times 10^{-3}$ and $\mathrm{B}\left(\mathrm{E} 1 ; 3 / 2^{-} \rightarrow 3 / 2^{+} \mathrm{gs}\right)=4.1 \times 10^{-4}$. The EEdf1 calculation yields $\mathrm{B}\left(\mathrm{E} 1 ; 1 / 2^{+} \rightarrow 3 / 2^{-}\right)=1.4 \times 10^{-2}$ and $\mathrm{B}\left(\mathrm{E} 1 ; 3 / 2^{-} \rightarrow 3 / 2^{+} \mathrm{gs}\right)=6.7 \times 10^{-3}$. The experimental values are $\mathrm{B}\left(\mathrm{E} 1 ; 1 / 2^{+} \rightarrow 3 / 2^{-}\right)>3 \times 10^{-2}$ and $\mathrm{B}\left(\mathrm{E} 1 ; 3 / 2^{-} \rightarrow 3 / 2^{+} \mathrm{gs}\right)=6.7_{-1.3}^{+2.1} 10^{-4}$.

Source: Adapted from Ref. [187].
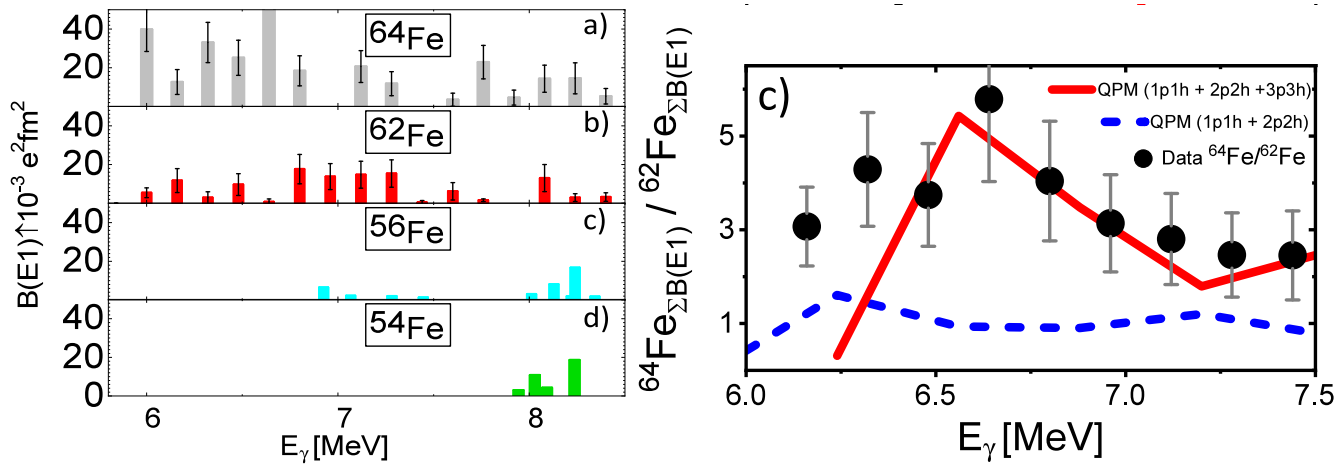

Fig. 44. Left panels : $\mathrm{B}(\mathrm{E} 1)$ values for $1^{-}$strength measured with AGATA for the radioactive ${ }^{62,64} \mathrm{Fe}$ nuclei. Right panel: the ratio of the summed $\mathrm{B}(\mathrm{E} 1)$ for the nuclei ${ }^{62,64} \mathrm{Fe}$ as a function of energy. The experimental data are shown with circles and the lines are calculation including (1p-1h) excitations (dashed line) and (3p-3h) states (full drawn line).

Source: Adapted from Ref. [55].

indicates that core excitations and deformation are key mechanisms in inducing the E1 enhancement. In addition, from these findings emerge the necessity of further studies to pin down the absolute E1 strength and its origin.

\subsubsection{Low-lying electric dipole $\gamma$-continuum for the unstable ${ }^{62,64} \mathrm{Fe}$ nuclei}

The $\gamma$-ray emission from the nuclei ${ }^{62,64} \mathrm{Fe}$ following Coulomb excitation at bombarding energy of $400-440 \mathrm{AMeV}$ was measured with AGATA with special focus on $E 1$ transitions in the energy region 4-8 MeV. The unstable neutron-rich nuclei ${ }^{62,64} \mathrm{Fe}$ were produced at the GSI laboratory and selected with the FRS fragment separator spectrometer.

The work was motivated by the search of electric dipole strength at energy below the neutron binding energy and to determine its evolution with neutron number. In the case of the stable Sn and Xe nuclei, having the longest chains of isotopes, their $E 1$ strength was found to be more fragmented and to have a marked increase with increasing neutron number. This resulted to be in rather good agreement with predictions from the Quasi Particle Phonon Model (QPM) [188], a theory which was expanded during the years to include couplings to complex configurations.

For the nuclei ${ }^{62,64} \mathrm{Fe}$, studied with AGATA [55], the search of $E 1$ strength below the neutron binding energy is particularly interesting for the modeling of the r-process where the $E 1$ decay is competing with the neutron capture. The used experimental approach consisted in the electromagnetic excitation (via virtual photon exchange) of these nuclei produced as radioactive beams. The excitation of dipole states requires the use of high bombarding energy implying very large Doppler shift ( $\beta=0.72$ at the energy of this experiment) corrections and thus the precise tracking of the $\gamma$-ray emitting particle is crucial as well as the accurate determination of the direction of the emitted $\gamma$-rays.

In the left panel of Fig. 44 the measured $\mathrm{B}(\mathrm{E} 1)$ strength in ${ }^{62,64} \mathrm{Fe}$ is shown in comparison with data of the less neutronrich isotopes ${ }^{54,56} \mathrm{Fe}$. One can see that the $\mathrm{E} 1 \mathrm{strength}$, having a continuum character, increases with neutron number. In particular, for the two nuclei ${ }^{62,64} \mathrm{Fe}$ the ratio of the B(E1) summed over energy (running sum) is found to be of the order 


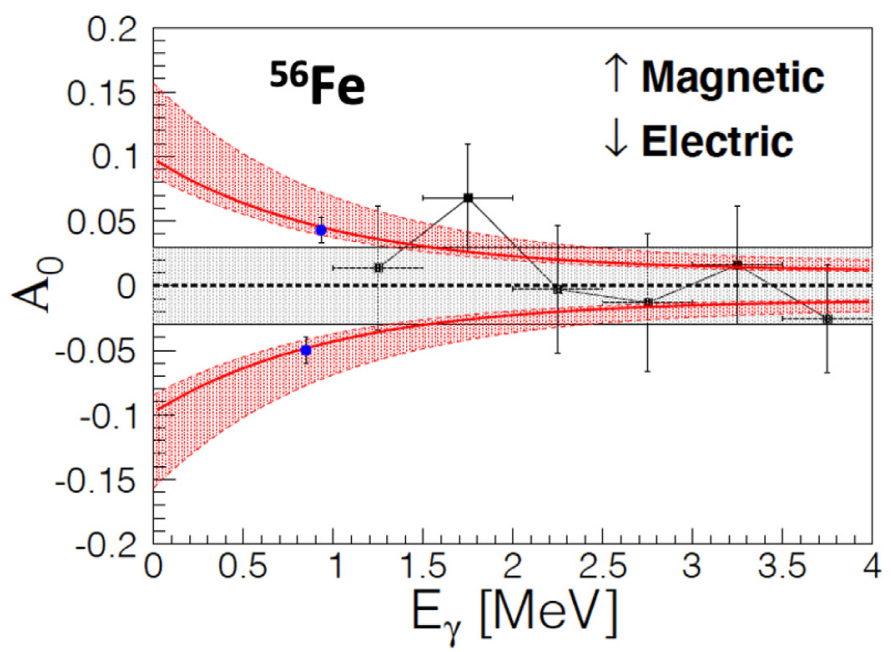

Fig. 45. Asymmetry of the Compton scattering for $\gamma$ transitions in ${ }^{56} \mathrm{Fe}$ nucleus. This asymmetry is positive for magnetic type transitions and is negative for electric type transitions (as indicated by the upward and downward arrows in the legenda). These data were obtained with an experiment performed with the GRETINA array. The points shown with blue circles correspond to known discrete transitions.

Source: Adapted from Ref. [189].

of 3 in average (see right panel of Fig. 44). This ratio was also calculated within the framework of QPM model [188]. This model is well suited to describe the $E 1$ strength at low energy and it has been widely used from the late 90 s to describe the extensive existing data of pygmy states from $\left(\gamma, \gamma^{\prime}\right)$ experiments. An important feature of this model is the inclusion of complex configurations by going beyond the simple one-particle one-hole (1p-1h). The QPM predictions, shown in comparison with the data in the right panel of Fig. 44), are indeed very different when only (1p-1h) states are included and to reproduce the data (3p-3h) configurations have to be also included. The ambition for the future is to go more in depth into this interesting problem by performing experiments with more statistics also in nuclei further away from stability.

In connection with the comparison of the $\mathrm{E} 1$ strength in the unstable neutron-rich Fe isotopes with the stable Fe nuclei it is important to recall here the rather recent results on the gamma strength for the ${ }^{56} \mathrm{Fe}$ nucleus [189] . These results are based on an experiment performed with the GRETINA array, which has allowed to extract the $\gamma$-ray strength function down to an energy less than $1 \mathrm{MeV}$. The experiment used the proton inelastic scattering at bombarding energy of $16 \mathrm{MeV}$ as a mechanism to populate excited states. The measurement of events in which the excitation energy information, deduced from the energy of the scattered protons, was obtained in coincidence with two gamma-rays, detected in AGATA, allowed the use of a model-independent technique. Indeed, the gamma strength was deduced from the measurement of the ratio of the intensity population of states of the same spin and parity (the chosen states were $2^{+}$and $4^{+}$). These ratios were measured as a function of excitation energy. The results are found to be in agreement with existing data of the strength function deduced from other measurements. In the region below $2 \mathrm{MeV}$ an enhancement was found in the strength function, whose angular distribution is consistent with a dipole radiation. The experiment allowed the additional measurement of the polarization of the emitted gamma-rays and the corresponding results are displayed in Fig. 45 . The measured polarization shows a small bias towards a magnetic character in the region of the enhancement. This interesting result opens perspectives for further work to investigate the nature of the upbending behavior below $3 \mathrm{MeV}$ [190].

\subsubsection{Quadrupole excitation of the neutron skin}

In the case of the ${ }^{124} \mathrm{Sn}$ nucleus an accumulation of the E2 strength in the energy interval 3-4.5 MeV was identified with the $\left({ }^{17} \mathrm{O},{ }^{17} \mathrm{O}^{\prime} \gamma\right)$ reaction [191], similarly to that seen with the $\left(\alpha, \alpha^{\prime} \gamma\right)$ and $(\gamma, \gamma)$ reactions [192]. The analysis of this strength was motivated by the obvious question on the possibility of having neutron skin excitations of multipolarities other than the dipole type. Promising candidates are low-energy $2^{+}$states. Theoretical predictions using the HartreeFock-Bogoliubov (HFB) and quasiparticle random phase approximation (QRPA) plus multiphonon approach [193] for the tin isotopic chain show a concentration of low-energy electric quadrupole strength located much below the isoscalar giant quadrupole resonance (ISGQR) which could represent a new nuclear excitation mode, the Pygmy Quadrupole Resonance $(\mathrm{PQR})$. This interpretation is based on the detailed analysis of spectroscopic features and transition rates. Thus, the microscopic structure of the QRPA $2^{+}$states with excitation energy less than about $E_{x}=5 \mathrm{MeV}$ in Sn nuclei with N/Z $>1.1$, is predominantly of neutron character, dominated by two-quasiparticle neutron states located close to the Fermi surface. An important part of the correlations is given by the quadrupole pairing interaction. The states are clustered in a comparatively small energy interval, thus giving rise to a neutron $\mathrm{PQR}$. The most convincing evidence for the connection between $\mathrm{PQR}$ and neutron pairing is the disappearance of the $\mathrm{PQR}$ in the double magic ${ }^{132} \mathrm{Sn}$ nucleus. In this connection, 

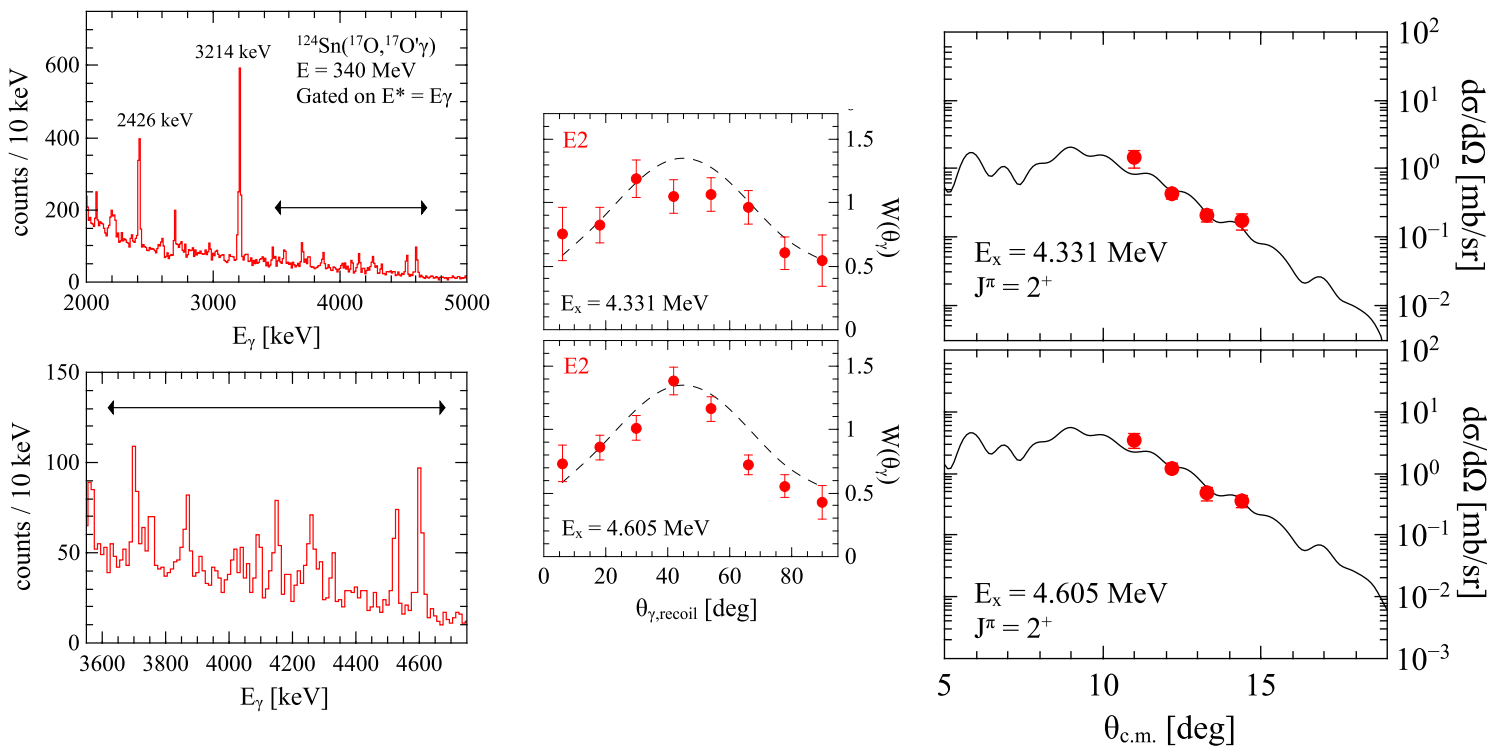

Fig. 46. Left panels: The top panel displays the spectrum measured with AGATA for the $\left({ }^{17} \mathrm{O},{ }^{17} \mathrm{O} \gamma\right)$ reaction on ${ }^{124} \mathrm{Sn}$, and the bottom panel gives an expanded view for the E2 transitions. Central panels: the angular distribution for the two new transitions at $4.331 \mathrm{MeV}$ (top) and $4.605 \mathrm{MeV}$ (bottom). Right panels: Inelastic scattering cross section for the ${ }^{124} \mathrm{Sn}\left({ }^{17} \mathrm{O},{ }^{17} \mathrm{O}^{\prime} \gamma\right)^{124} \mathrm{Sn}^{*}$ at $340 \mathrm{MeV}$ for the $2^{+}$states at $4.331 \mathrm{MeV}$ (top) and $4.605 \mathrm{MeV}$ (bottom). The error bars are the statistical errors. The lines show DWBA calculations with the standard phenomenological form factor and assuming $\mathrm{M}_{n} / \mathrm{M}_{p}=\mathrm{N} / \mathrm{Z}$.

Source: Adapted from Ref. [191].

the PQR states contain information on the structure of the valence shells and of their evolution with the nuclear mass. Furthermore, the correlation between the $\mathrm{PQR}$ and the neutron or proton skin thickness manifests itself via a transition from a neutron $\mathrm{PQR}$ to a proton $\mathrm{PQR}$ in ${ }^{124} \mathrm{Sn}$.

In the AGATA experiment several $2^{+}$states were populated and the subsequent $\gamma$ decay was observed in the energy region between 3.5 and $5 \mathrm{MeV}$. In principle, in this region one expects that transitions of E1, M1 and E2 types occur. Before the work based on AGATA data, only a few $2^{+}$states in ${ }^{124}$ Sn were identified via photon, proton, and $\alpha$-scattering as well as $\beta$-decay experiments. With AGATA several $2^{+}$states above $3.5 \mathrm{MeV}$ and below the neutron separation threshold were identified via the measurement of their $\gamma$-decay energy and angular distribution. The measured spectrum and a particular portion of it, containing the new transitions, are shown in the two left panels of Fig. 46, while two of the measured angular distributions are shown in the two central panels of the same figure. The E2 character is clearly identified by the angular distributions of the peak intensity.

Since the population cross section of the different excited states was measured at different angles of the scattered ${ }^{17} \mathrm{O}$ ions a DWBA analysis of the data was performed. The calculated differential cross sections for the two new states at 4.605 and $4.331 \mathrm{MeV}$ are shown in the two right panels of Fig. 46 in comparison with the data. In the calculations of the excited state cross sections the optical model parameters obtained from the analysis of the elastic scattering were used. For the $2^{+}$states the vibrational collective form factor was used and it was assumed that the ratio of the neutron and proton transition matrix elements is $\mathrm{M}_{n} / \mathrm{M}_{p}=\mathrm{N} / Z$. This assumption is supported by calculations and Ref. [193]. A good agreement is found between data and calculations.

The results for the population cross section for the $2^{+}$states up to $5 \mathrm{MeV}$ are shown in the left central panel of Fig. 47 in comparison with data from the $\left(\gamma, \gamma^{\prime}\right)$ reaction (top panel) and with the predictions within the Quasiparticle-Phonon Model (QPM) described in [193] (bottom panel). On the right part of Fig. 47 a table is given reporting the known and the extracted $\mathrm{B}(\mathrm{E} 2)$ values. Altogether the experimental and theoretical results show the presence of a number of $2^{+}$states grouping together in the energy region 3-5 MeV supporting the prediction of the HFB+QPM model [193]. This quadrupole strength clustering appears to be similar to the known PDR states at 5-7 MeV. The microscopic analysis of these $2^{+}$states reveals that they have a unique structure closely connected with excitation of the neutron skin. One cannot exclude a much more complicated situation in which the low-lying E1 strength mixes with "PQR" states. In the future it will be important to obtain information on the transition densities of these states, and to study other isotopes to learn more on the quadrupole degree of freedom of the neutron skin.

\subsection{The GDR in hot nuclei}

The $\gamma$-decay from the giant dipole resonance built on excited states was extensively studied both with experiments and with theory during the past years. It was found that the GDR vibration is a useful tool to probe properties of hot 

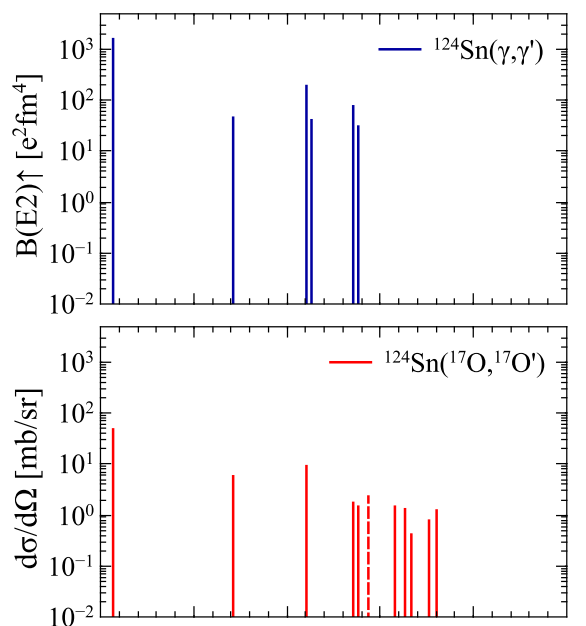

\begin{tabular}{lccc}
\hline$E$ & $B(E 2) \uparrow$ & $\frac{d \sigma}{d \Omega_{\mathrm{c.m} .}}$ & $B(E 2) \uparrow$ extracted \\
{$[\mathrm{MeV}]$} & {$\left[e^{2} \mathrm{fm}^{4}\right]$} & {$[\mathrm{mb} / \mathrm{sr}]$} & {$\left[e^{2} \mathrm{fm}^{4}\right]$} \\
\hline 1.132 & 1660 & $47.5(8.3)$ & - \\
2.426 & 95 & $5.63(83)$ & - \\
3.214 & 275 & $9.3(1.3)$ & - \\
3.710 & 75 & $1.78(30)$ & - \\
3.762 & 60 & $1.49(24)$ & - \\
$3.864-3.888$ & - & $2.36(37)$ & $90(14)$ \\
4.156 & - & $1.43(24)$ & $60(10)$ \\
4.264 & - & $1.27(22)$ & $55(10)$ \\
4.331 & - & $0.43(7)$ & $20(3)$ \\
4.529 & - & $0.78(15)$ & $40(8)$ \\
4.605 & - & $1.23(21)$ & $55(9)$ \\
\hline \hline
\end{tabular}

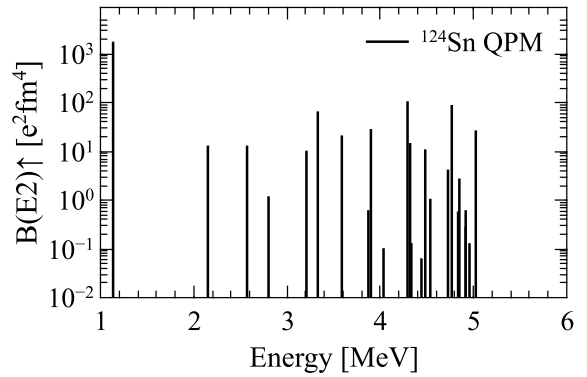

Fig. 47. Left panels: B(E2) values for $2^{+}$states in ${ }^{124} \mathrm{Sn}$ from the $\left(\gamma, \gamma^{\prime}\right)$ reaction (top panel) and from calculations within the quasiparticle-phonon model described in [193] in the bottom panel. The population cross section for the $2^{+}$states in ${ }^{124} \mathrm{Sn}$ obtained with the ( ${ }^{17} \mathrm{O}$, ${ }^{17} \mathrm{O}^{\prime} \gamma$ ) reaction is given in the central panel. There the dashed line indicates that the energy of that state was tentatively assigned. Right panel: Table with $\mathrm{B}$ (E2) values for the different $2^{+}$states reported in the first column. The last column gives the values of the B(E2) assumed for the DWBA calculations.

Source: Adapted from Ref. [191].

nuclear systems since it occurs on a timescale which is extremely short. In particular, it is used to study the properties such as shape and deformation of hot nuclei and their evolution with excitation energy and angular momentum. After the first evidence of the excitation of the GDR state built on a compound nucleus (CN), numerous examples of "hot" nuclei were studied in a large interval of angular momentum and excitation energy. The experiments are based on the detection of high energy gamma rays emitted in heavy-ion fusion reactions. The overarching picture deduced from the existing experiments is that the measured width of the GDR state built on excited nuclei is much larger than that of the GDR built on the ground state.

The width of the GDR reflects its damping mechanism which is mainly due to the coupling to complex np-nh states through $2 \mathrm{p}-2 \mathrm{~h}$ doorway states. This damping process, also known as "collisional damping", gives rise to what we define as "the intrinsic width" of the GDR and it is expected not to depend on temperature in a sizeable way. Because the observed width was found in general to monotonically increase with temperature and angular momentum, it became clear that a new damping mechanism was acting, and this was called "in-homogeneous" damping, which is due to the deformation of the nucleus. Indeed, the width of the GDR built on excited states is due to these two different damping mechanisms at play (see e.g. [194]). The theoretical calculations of the "in-homogeneous" damping consider the coupling of the dipole vibration to the quadrupole deformation and use the scenario of thermal shape and orientation fluctuations to predict the GDR width. This "model" explains rather well the experimental data of the GDR width for compound nuclei with temperature in the window $1.5 \sim 3.0 \mathrm{MeV}$ and with angular momentum lower than $50 \hbar$ (see [36] and references therein).

An experiment was performed with AGATA at LNL in which the $\gamma$ decay from the GDR in two hot rotating nuclei in the mass region $A=80$ was measured [195] and [196]. The main motivation of the experiment was to address the problem of the isospin mixing (see Section 7.1.2) in nuclei. However, to extract the isospin mixing in hot nuclei from the GDR $\gamma$ decay it is important to check first how the GDR width emerges from the data, since the width information is used as a fixed parameter in the isospin mixing analysis. The results of this AGATA experiment are summarized below.

\subsubsection{The width of the GDR in hot rotating ${ }^{81} \mathrm{Rb}$ nuclei}

The low and high energy $\gamma$-rays emitted in the reaction ${ }^{37} \mathrm{Cl}^{44} \mathrm{Ca}$ at $\mathrm{E}_{\text {beam }}=95 \mathrm{MeV}$ were measured with a set up consisting of the AGATA system coupled to an array of large-volume $\mathrm{LaBr}_{3} 3$ :Ce detectors, named $\mathrm{HECTOR}^{+}$. The low energy 

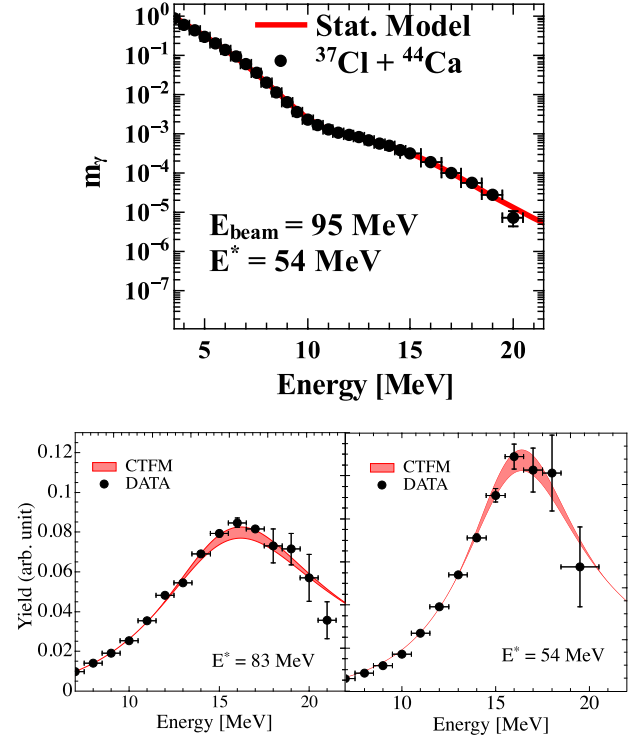

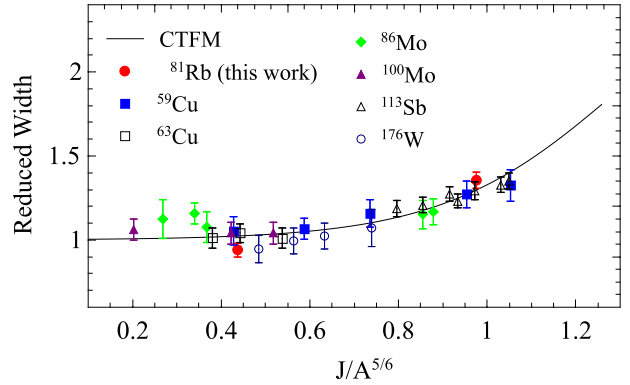$$
\mathrm{J} / \mathrm{A}^{5}
$$

Fig. 48. Left panels: In the top panel the high-energy $\gamma$-ray spectrum measured for the reaction ${ }^{37} \mathrm{Cl}+{ }^{44} \mathrm{Ca}$ at $95 \mathrm{MeV}$ is shown with full circles in comparison with the best-fitting statistical model calculation (red lines) (from [196]). In the two bottom panels the linearized $\gamma$-ray spectra (see text) are compared with theoretical calculations performed using the CTFM model, where the red bands reflect the uncertainty on the value of the temperature. Right panel: The curve gives the GDR reduced-width within the framework of the CTFM model and from fit of the data. The experimental results for different nuclei are shown with symbols. The data for the ${ }^{81}$ Ru nucleus are shown with red circles.

Source: Adapted from Refs. [195] and [196].

$\gamma$-ray spectra were used to deduce the population of the different residual nuclei and this is an important information providing a valuable cross check for the analysis of the high energy $\gamma$-ray spectra with the statistical model.

In the left-top panel of Fig. 48 the high energy $\gamma$-ray spectrum measured with the fusion evaporation reaction, forming a compound nucleus at excitation energy $\mathrm{E}^{*}=54 \mathrm{MeV}$, is shown in comparison with the statistical model calculation including the GDR strength function with a line shape of Lorentzian type. The calculations were folded with the detector response function and normalized to the experimental data at around $5 \mathrm{MeV}$. The GDR parameters were derived from the best fit to the data in the region between 8 and $15 \mathrm{MeV}$. Due to the exponential nature of the spectra, the standard $\chi^{2}$ is not a suitable quantity because of its weak sensitivity to the low-yield part of the spectrum. For this reason, the fit minimization was applied to a figure of merit (FOM) defined as the standard $\chi^{2}$ over the number of counts for each bin. In this way, the sensitivity to the low-yield part of the spectrum is much increased.

In the left-bottom panels of Fig. 48 the quantity Yield $\left(\mathrm{E}_{\gamma}\right)=\mathrm{F}\left(\mathrm{E}_{\gamma}\right) \times \mathrm{Y}_{\gamma}^{\text {exp }}\left(\mathrm{E}_{\gamma}\right) / \mathrm{Y}_{\gamma}^{\text {cal }}\left(\mathrm{E}_{\gamma}\right)$ is shown. In this expression $\mathrm{Y}_{\gamma}^{\exp }\left(\mathrm{E}_{\gamma}\right)$ and $\mathrm{Y}_{\gamma}^{\text {cal }}\left(\mathrm{E}_{\gamma}\right)$ are the measured and calculated yields while $\mathrm{F}\left(\mathrm{E}_{\gamma}\right)$ is the best fitting Lorentzian function. The left(right) panel shows data corresponding to an excitation energy of $83 \mathrm{MeV}(54 \mathrm{MeV})$ and the error bars are the statistical errors. By increasing the excitation energy from 54 and $83 \mathrm{MeV}$ and the average spin from 17 to 38 the GDR width is found to change from $7 \pm 0.2 \mathrm{MeV}$ to $10.8 \pm 0.2 \mathrm{MeV}$. At $T=0$ the GDR width is $4.5 \mathrm{MeV}$. The GDR centroid energy is $16.8 \mathrm{MeV}$ in the ground state, $16.4 \pm 0.2 \mathrm{MeV}$ at $\mathrm{E}_{\exp }=54 \mathrm{MeV}$, and $16.2 \pm 0.2 \mathrm{MeV}$ at $\mathrm{E}^{\text {exp }}=83 \mathrm{MeV}$ so that it does not significantly change with temperature, as it was found for many other nuclei at different values of mass, excitation energy and angular momentum.

As mentioned at the beginning of this subsection the thermal fluctuation model is used to describe the GDR width in hot rotating nuclei. A large number of calculations were made as function of the mass number $A$, of the nuclear temperature $\mathrm{T}$ (derived from excitation energy using the level density parameter), and of the angular momentum J. Using the many existing data it was possible to parametrize the width of the thermal fluctuation model (TFM) as described in [197] and [198]. The latest parametrization (called Critical Temperature Thermal Fluctuation Model, CTFM) is reported in [197] and [198] where reference is given also to the earlier ones.

This latest parametrization of the GDR width is shown in right panel of Fig. 48. In particular, the plotted quantity "Reduced Width" is related, via an exponential factor, to the ratio of the measured total width (e.g. $7 \mathrm{MeV}$ for the datum at $\mathrm{E}_{e x}=54$ ) with a calculated quantity at total angular momentum $J=0$ (for the details of the used expressions see [195]). The values of the "Reduced Width" from the general fit (not including the ${ }^{81} \mathrm{Ru}$ data) are shown in the right panel of Fig. 48 with the continuous curve. The obtained values for the ${ }^{81} \mathrm{Rb}$ nucleus at excitation energy $\mathrm{E}_{e x}=54$ and $83 \mathrm{MeV}$ are shown with the red circles in comparison with other data and calculations.

The AGATA point at $\mathrm{E}_{e x}=54 \mathrm{MeV}$ is well placed in the general behavior of the existing data. The result for the nucleus ${ }^{81} \mathrm{Rb}$ at excitation energy $\mathrm{E}_{e x}=54 \mathrm{MeV}$ is important not only because it confirms the existing trend of the GDR width but also because it is used in the analysis for the determination of the isospin mixing described in Section 7.1.2. 


\section{Shapes and high-spin phenomena}

While the quest for a uniform description of nuclear structure is promoted by latest results from exotic nuclei far from stability complementary routes are followed by detailed spectroscopy along the valley of stability. Innovative experimental techniques like $\gamma$-ray tracking allow delineation of shapes and high-spin phenomena in an unprecedented way. AGATA provided prime examples for new results concerning the shape of nuclei, isomerism and high-spin features that were confronted with latest theoretical advances.

The occurrence of very elongated prolate nuclear shapes with an axis ratio of 2:1 is a major facet of nuclear structure for over 40 years. Superdeformation was discovered in the guise of fission isomers [199,200] and was followed by its manifestation at high spin in the form of rotational bands at mass numbers 150, and 190 [201,202]. Later superdeformed states were found also along the $\mathrm{N}=\mathrm{Z}$ line, where protons and neutrons occupy similar orbitals. Especially the doubly magic nuclei ${ }^{56} \mathrm{Ni}$ [203] and ${ }^{40} \mathrm{Ca}$ [204] feature dramatic examples of shape coexistence with spherical and superdeformed configurations. The discovery of these states came along with the observation of intense discreteenergy linking transitions between the structures in the second superdeformed and the first spherical potential well, including particle decays [203,205]. This allows for firm spin and parity assignments for the superdeformed bands in most cases. In addition, these nuclei are accessible to different theoretical approaches that include shell-model calculations, quantum Monte Carlo shell-model descriptions, and a variety of mean-field approaches. Especially, the low mass nuclei provide an ideal testing ground to compare and confront the complementary models with hitherto known aspects of superdeformation. Moreover, the AGATA experiment allowed to study a new population mechanism for superdeformed states via Coulomb excitation allowing very precise determination of their quadrupole collectivity.

Detailed nuclear-structure data of very neutron-deficient nuclei extend to nuclei at or even beyond the proton-drip lines because very neutron-deficient compound systems are formed at the extremes of angular momentum and excitation energies using fusion-evaporation reactions with stable beam-target combinations. A considerable and not negligible number of only slightly neutron-rich nuclei near the valley of stability are not accessible due to a lack of suitable stable beam-target combinations for fusion-evaporation reactions. In these cases, the observation of high-spin structures, like yrast sequences up to energies where backbending occurs, new features such as shape coexistence or deformed collective bands is quite demanding. It was achieved in a series of AGATA experiments exploiting reaction products from MNT reactions and highly selective particle spectrometers like PRISMA at LNL or VAMOS++ at GANIL.

The $50 \leq Z, N \leq 82$ nuclei outside the doubly-magic nucleus ${ }^{132} \mathrm{Sn}$ are expected to show predominantly single-particle characteristics. Metastable, long-lived nuclear excited states i.e. isomers, are well known and important signatures for sudden changes in the underlying nuclear structure. Small overlap and large differences between the initial and final wave functions of the states involved in the decay cause a transition hindrance. Hence, it is of particular interest to study the evolution of isomers and states built on top as a function of the gradual filling of shell-model orbitals along shell closures. Realistic effective shell-model interactions derived from modern nucleon-nucleon potentials as well as phenomenological interactions are available for the description of the nuclear structure northwest of ${ }^{132} \mathrm{Sn}$.

Similar AGATA experiments were performed in the vicinity of the $Z=50$ shell closure, near $N=82$ [206,207]. Here a structural similarity exists between high-spin isomeric states in $\operatorname{In}(Z=49)$, Sn $(Z=50)$, and Sb $(Z=51)$ isotopes. Results of a detailed investigation of the spin-orbit partner orbitals, $\pi g_{9 / 2}$ and $\pi g_{7 / 2}$, enclosing the magic gap are described in the following chapter 7, Section 7.3.

Early realistic interactions for the description of protons and neutrons in the valence space of the $0 g_{7 / 2}, 1 d_{5 / 2}, 1 d_{3 / 2}$, $2 s_{1 / 2}$, and $0 h_{11 / 2}$ (gdsh) orbitals date back to the early 1980s (see Fig. 53). For example, the N82 interaction by Kruse and Wildenthal was derived from a least-squares fit to binding energies with a surface-delta interaction as a starting point and attempted to describe proton excitations along $\mathrm{N}=82$ isotones north of ${ }^{132} \mathrm{Sn}$ [208]. New interactions are expected to provide a comprehensive description of nuclear-structure features such as excitation energies, transition strengths and magnetic moments. The entanglement of single-particle and collective modes towards the mid-shell regime is another challenge. Recent progress and present-day realistic interactions are available.

The SN100PN interaction is a realistic interaction by Brown et al. for the description of the gdsh orbitals in the valence space outside the ${ }^{100} \mathrm{Sn}$ core between the magic numbers 50 and 82 . The interaction consists of separate neutron-neutron, proton-proton, and proton-neutron effective interactions plus a Coulomb-repulsion part. In particular, the predictive power of the neutron-neutron and the proton-proton part were thoroughly tested in experiments. However, detailed and stringent tests of the proton-neutron part are still pending. Another independent approach is the pair-truncated shell model, which is similar to the concept of the interacting boson model.

Odd-mass and doubly-odd nuclei are treated by coupling additional unpaired nucleons to the nuclear states calculated for the even-even nuclei. A plethora of theoretical results for nuclei ranging from Sn to Ba, including excitation energies, transition rates, and magnetic moments, were obtained by Teruya et al. [209] with a phenomenological interaction called PQM130 (Pairing+QQ+Multipole for mass region 130). The effective Hamiltonian comprises in a similar way neutron-neutron, proton-proton, and neutron-proton interactions, respectively. The interaction is constructed from a pairing-plus-quadrupole interaction that consists of spherical single-particle energies, the monopole-pairing (MP) interaction, the quadrupole-pairing (QP) interaction, as well as the quadrupole-quadrupole (QQ) interaction. It is combined with newly introduced higher multipole-pairing (HMP) interactions.

A recent development by Gniady, Caurier, and Nowacki is the GCN5082 interaction for the gdsh valence space for both protons and neutrons [210]. Like the SN100PN interaction, the interaction is derived from a realistic G matrix 
based on the CD-Bonn potential. Empirical corrections are added to the monopole part and certain pairing and other multipole matrix elements of the original G matrix. In this way, a set of about 320 low-spin states in 87 nuclei within the $50 \leq Z, N \leq 82$ region was reproduced within a remarkable root-mean-square deviation. Among others, the interaction has been employed for shell-model studies of neutrino-less decays in even-even Sn, Te and Xe isotopes, high-spin structures of ${ }^{134} \mathrm{Xe}$, the analysis of electromagnetic transition rates for the understanding of mixed-symmetry states along the $\mathrm{N}=80$ isotones, and even in studies of dark-matter scattering on xenon isotopes.

Surprisingly and in spite of several experimental efforts, detailed information on high-spin properties of $50 \leq Z, N \leq$ 82 nuclei close to stability is still scarce. A systematic study of high-spin states in even-even nuclei, as well as odd-A nuclei in this region, was undertaken with AGATA at LNL.

In atomic nuclei the deformed shape is due to the complex interplay between the residual nucleon-nucleon interactions driving towards deformation and the shell gaps that tend to restore the spherical shape. The study of the nuclear shape evolution along an isotopic chain opens a window on the underlying microscopic force and is an important testing ground for nuclear models. AGATA contributed to the understanding of this type of questions in neutron-rich nuclei with mass numbers around $A=96, A \approx 200$ and $A=240$.

One region of the nuclear chart, where oblate, prolate, $\gamma$-soft, and spherical shapes are observed and predicted is the tungsten-osmium-platinum region with $A \approx 190$. A prolate-to-oblate shape transition is predicted to appear when moving towards the $N=126$ shell closure, where the spherical shape should be restored. For platinum and osmium isotopes such a shape transition occurs while passing through nuclei having a $\gamma$-soft potential. However, the path to spherical shapes is not yet fully delineated.

The heavy nuclei beyond the last doubly magic nucleus ${ }^{208} \mathrm{~Pb}$ in the actinide region from radium to nobelium show a variety of shapes in the ground state and at higher excitation energies [211]. Besides a pronounced ground-state deformation in the quadrupole degree of freedom, also higher multipole orders are relevant and necessary to understand the basic properties of these nuclei [212-215]. Especially, this is relevant for the extrapolation into the region of the heaviest elements, where a reduced deformation beyond the mid-shell region is a clear indicator for the next magic number. At this point not only the deformation as a function of proton number, but also its dependence on the neutron number is of high interest for the understanding of the neutron shell closures of super-heavy elements.

\subsection{Shape changes around doubly magic ${ }^{40} \mathrm{Ca}$}

Highly deformed nuclei of elongated shapes that resemble an ellipsoid with a 2:1 major to minor axes ratio, were first observed in fission or shape isomers. This discovery was followed by the observation of superdeformed (SD) rotational bands in the rare-earth region. A particularity of these structures, which are found to occur at high spin, was that their linking to the ground-state band was difficult to establish $[205,216]$. The phenomenon of superdeformation became a challenge for both experiment and nuclear structure theory, and soon very similar structures were found in other mass regions.

The AGATA publications [217-219] report on an experiment to study the electromagnetic structure of ${ }^{42} \mathrm{Ca}$. The experiment exploited for the first time Coulomb excitation to study superdeformed states because the low-lying bandhead of the sideband in ${ }^{42} \mathrm{Ca}$ is at an excitation energy of $1837 \mathrm{keV}$ (see level scheme in Fig. 49). This is considerably lower than its counterparts in the neighboring $\mathrm{Ca}$ and $\mathrm{Ar}$ isotopes allowing to populate this structure via Coulomb excitation. In the very first experiment using the AGATA array at all, the superdeformed structure in ${ }^{42}$ Ca was populated following Coulomb excitation of the ${ }^{42} \mathrm{Ca}$ beam on ${ }^{208} \mathrm{~Pb}$ and ${ }^{197} \mathrm{Au}$ targets. From the measured $\gamma$-ray intensities a complete set of electromagnetic matrix elements between the observed states was obtained, including spectroscopic quadrupole moments and relative signs of transitional E2 matrix elements. Those, in turn, were interpreted using the quadrupole sum rules formalism leading to the conclusion that the spherical ground state of ${ }^{42} \mathrm{Ca}$ exhibits large fluctuations into the $\beta-\gamma$ plane. The excited structure has a large quadrupole deformation of $\beta=0.43(4)$ for $0_{2}^{+}$, comparable to those measured for other superdeformed bands in this mass region. The moment of inertia, which is proportional to the quadrupole deformation parameter, is large and similar to those in the SD bands in both ${ }^{36} \mathrm{Ar}$ and ${ }^{40} \mathrm{Ca}$.

Moreover, the quadrupole sum rules method was applied to the obtained E2 matrix elements from the GOSIA analysis in order to extract information on the charge distribution of the nucleus in specific states. The results obtained for the low-lying states in ${ }^{42} \mathrm{Ca}$ were published in Ref. [217,218]. The quadrupole sum rules method is based on the fact that the electric multipole transition operator $E(\lambda=2, \mu)$ is a spherical tensor and it can be represented using two parameters: $\mathrm{Q}$, the overall quadrupole deformation parameter equivalent to the elongation parameter $\beta$ in Bohr's model, and $\delta$, which is related to the triaxiality parameter $\gamma$. The expectation values of the quadrupole rotational invariants $\left\langle Q^{2}\right\rangle$ and $\left\langle Q^{3} \cos (3 \delta)\right\rangle$ which describe the deformation of individual states in both the intrinsic and laboratory frames are determined using the set of E2 matrix elements by an expansion over all possible intermediate states. The $\left\langle Q^{2}\right\rangle$ and $\left\langle Q^{3} \cos (3 \delta)\right\rangle$ invariants were converted to the $\beta$ and $\gamma$ collective model deformation parameters, as explained in detail in Ref. [220]. The $\beta=0.43(4)$ and $\gamma=13\left({ }_{-6}^{+5}\right)^{\circ}$ deformation parameters obtained in this way for $0_{2}^{+}$show that the sideband has a slightly triaxial superdeformed shape. This result provided the first experimental evidence for non-axial character of superdeformed structures around $A \approx 40$.

The obtained experimental results were discussed in the context of microscopic calculations performed with both shell-model and beyond-mean-field (BMF) model approaches. Moreover, the generator coordinate method (GCM) was exploited with deformed-basis antisymmetrized molecular dynamics (AMD) wave functions. 

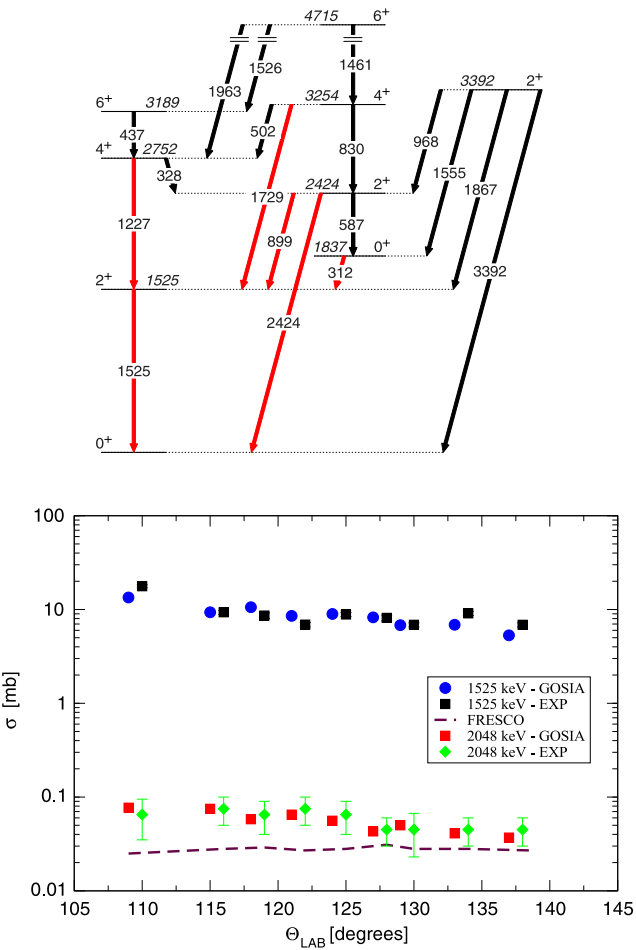
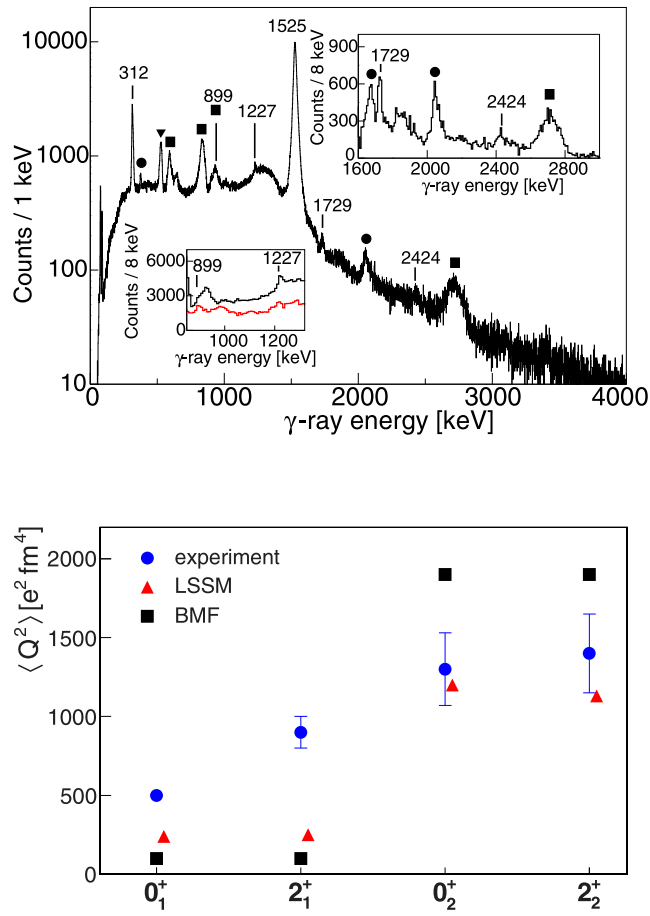

Fig. 49. Top-left panel: A portion of the level scheme of ${ }^{42} \mathrm{Ca}$ used in the data analysis to obtain the quadrupole moment. The transition seen in the AGATA experiment are shown in red. Top-right panel: The $\gamma$-ray spectrum observed in the ${ }^{42} \mathrm{Ca}+{ }^{208} \mathrm{~Pb}$ Coulomb excitation experiment was measured in coincidence with back-scattered particles. Low-lying excited states allowed the study of superdeformed structures built on top of the $0_{2}^{+}$state at an excitation energy of $1837 \mathrm{keV}$. The square, circle, and triangle symbols mark transitions from ${ }^{208} \mathrm{~Pb},{ }^{43} \mathrm{Ca}$ and $511 \mathrm{keV}$, respectively. The transitions marked in the level scheme red correspond to observed peaks in the $\gamma$-ray spectrum. Spectrum and level scheme are taken from [217] Bottom-left panel: Measured (calculated) cross section for the $1525 \mathrm{keV}$ transition in ${ }^{42} \mathrm{Ca}$ in blue circles (black squares) and for the $2048 \mathrm{keV}$ transition in ${ }^{43} \mathrm{Ca}$ in green diamonds (red squares). Bottom-right panel: Values of the quadrupole rotational invariant $\left\langle Q^{2}\right\rangle$ for the $0_{1,2}^{+}$and $2_{1,2}^{+}$states shown with circles, triangles and squares for the AGATA experiment, theory LSSM, theory BMF, respectively.

Source: Adapted from [218].

Shell-model calculations were performed using the SDPF.MIX interaction in the sdpf model space for neutrons and protons, with a virtual ${ }^{28} \mathrm{Si}$ core. This interaction has proven successful in describing properties of the superdeformed bands in ${ }^{40} \mathrm{Ca}$ and ${ }^{36} \mathrm{Ar}$ and reproducing ground-state magnetic moments of ${ }^{49,51} \mathrm{Ca}$ and quadrupole moments of ${ }^{47,49,51} \mathrm{Ca}$. The new results show very good agreement with data for the energies of excited states calculated within the shell model. The E2 matrix elements in the yrast band are underestimated, and those in the sideband overestimated with respect to experiment indicating mixing between the two bands. The experimental M1 transition strengths are well reproduced by the calculation. The quadrupole properties of the lowest non-yrast $2^{+}$states show the expected collective aspects: the spectroscopic quadrupole moment $Q_{s}\left(2_{3}^{+}, K=2\right)$ is nearly equal to $Q_{s}\left(2_{2}^{+}, K=0\right)$, and they have opposite signs. The $Q_{s}\left(3_{1}^{+}, K=2\right)$ value is close to zero $\left(0.14 \mathrm{efm}^{2}\right)$ and the low-lying $3_{1}^{+}$state is connected by a strong transition to the $2_{3}^{+}$ state. Furthermore, the intrinsic quadrupole moments $Q_{0}$ were derived from calculated in-band E2 matrix elements.

The quadrupole properties of the excited states calculated in the laboratory frame were related to the nuclear shape in order to investigate the nature of observed structures, in particular that of the $\gamma$ band. The $\beta$ and $\gamma$ deformation parameters for the $0_{1,2}^{+}$states in ${ }^{42} \mathrm{Ca}$ were derived from the E2 matrix elements obtained in the shell-model calculation using the Davydov-Filipov geometric model, Kumar and Cline's sum-rule approach, and constrained Hartree-Fock in the shell-model basis (CHFSM). In the Davydov-Filipov model, the $\gamma$ angle can be extracted from the ratio $B\left(E 2,2_{\gamma}^{+} \rightarrow\right.$ $\left.2^{+}\right) / B\left(E 2,2_{\gamma}^{+} \rightarrow 0^{+}\right)$, and the $\beta$ deformation parameter from the $Q_{0}$ intrinsic quadrupole moment. Dynamic deformation of the ground state were given in [217], hence $\beta$ and $\gamma$ parameters presented for the $0_{1}^{+}$state are understood as the mean values of the deformation.

The deformation parameters obtained using the three methods are consistent for the $0_{2}^{+}$state, and they are in a good agreement with the experimental value. However, for the ground state the obtained $\beta$ parameters are close to zero using the Davydov-Filippov model and CHFSM, while the sum rules yield a much larger value, closer to what is observed experimentally. The discrepancy is discussed in Ref. [217] and related to the fact that the deformation of the $0_{2}^{+}$state has a static character, while the ground state exhibits large fluctuations about a spherical minimum. 

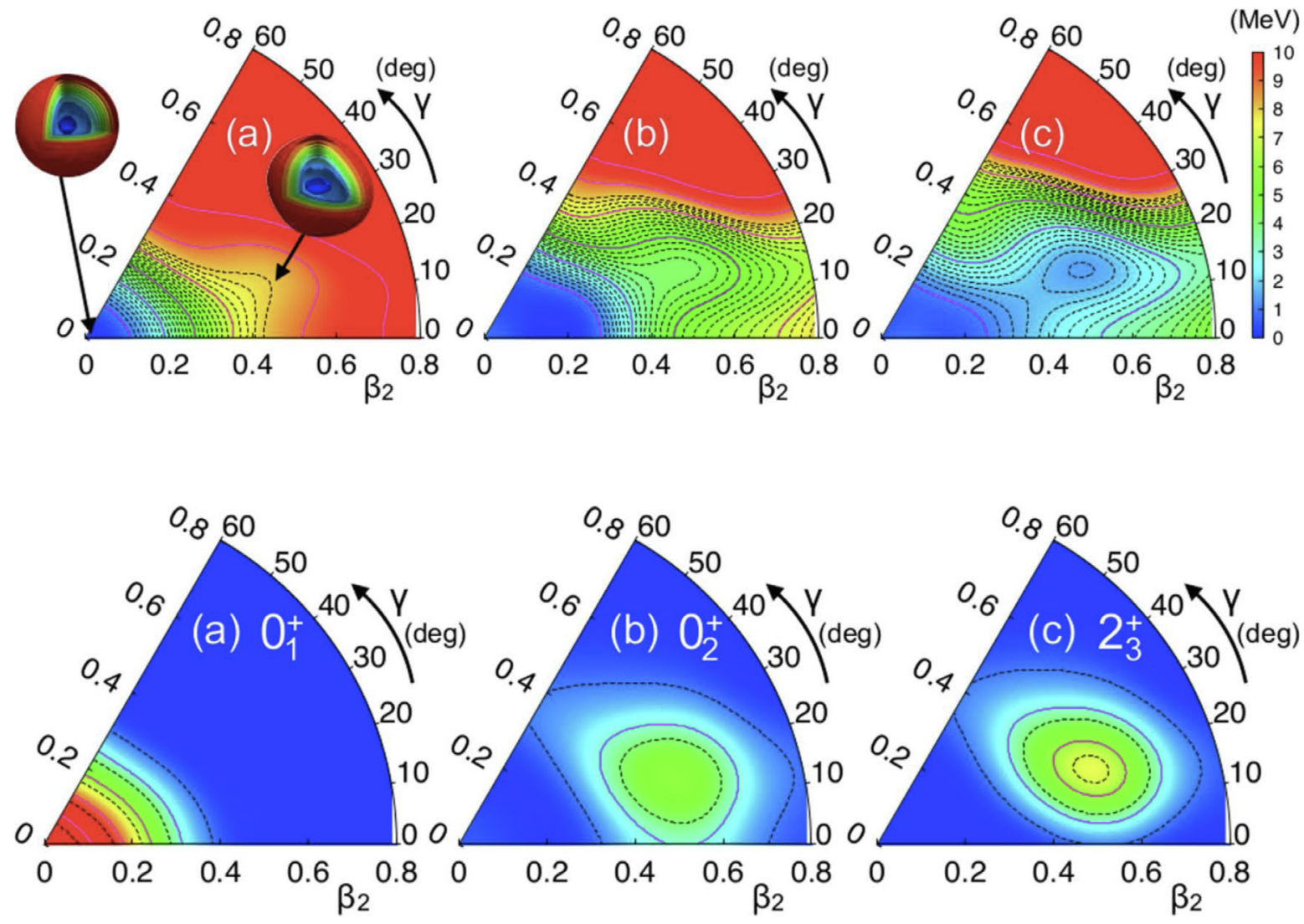

Fig. 50. Top panels: Potential-energy surfaces computed for ${ }^{42} \mathrm{Ca}$ with the Gogny D1S interaction and (a) particle number variation-after-projection (PN-VAP); (b) particle number and angular momentum projection (PNAMP) with $J=0$; and (c) PNAMP with $J=2$. Energies in each plot are normalized to that in its minimum and contour lines are separated by $0.25 \mathrm{MeV}$ (dashed lines) and $2 \mathrm{MeV}$ (solid lines), respectively. Inset in (a): Spatial densities corresponding to each minimum found in PN-VAP calculations. : Collective wave functions for the three $0_{1}^{+}$, $0_{2}^{+}$and $2_{3}^{+}$bandhead states obtained with BMF calculations with the Gogny D1S interaction. Red (blue) regions represent large (small) contributions to the wave functions. Source: Figures taken from [218].

The BMF calculations employed the symmetry-conserving configuration mixing (SCCM) method using the Gogny D1S interaction to define the corresponding energy density functionals. In the SCCM method the nuclear states are obtained as linear combinations of particle number and angular momentum projected mean-field states. The coefficients of the linear combination are calculated self-consistently following the generator coordinate method (GCM). The mean-field states are found by solving the particle-number variation-after-projection (PN-VAP) Hartree-Fock-Bogolyubov (HFB) equations with constraints in the quadrupole operators parametrized by $\beta_{2}, \gamma$. The PN-VAP energy defined in the $\beta_{2}, \gamma$ plane yields qualitative information on the quadrupole properties of the nucleus under study. In Fig. 50(top panel) the potential energy surface (PES) is plotted for $J=0,2$ configurations. The SCCM calculation includes the shape mixing within the GCM framework. As a result, the electromagnetic matrix elements in the laboratory frame are obtained for the lowest excited states which are grouped into three bands built on top of the $0_{1}^{+}, 0_{2}^{+}$and $2_{3}^{+}$states, respectively. The ground-state band shows small E2 transition probabilities and spectroscopic quadrupole moments, as expected for a spherical semi-magic configuration. In contrast, larger in-band transitions are predicted in the second and third bands. The underlying shapes of the states belonging to the three bands were obtained by analyzing the collective wave functions (CWFs) of the band heads. Fig. 50(bottom panel) shows the results for the three states. The CWFs represent the weights of each $\beta_{2}, \gamma$ deformation in the nuclear states obtained within the SCCM framework. The most important contribution to the ground state comes from the spherical point and its surroundings Fig. 50(bottom panel, a). For the states belonging to the second band the most relevant shapes are found around $\beta_{2}, \gamma=\left(0.55,20^{\circ}\right)$, consistent with the strong electromagnetic transitions and negative spectroscopic quadrupole moments obtained in the calculations. The third band shows CWFs similar to those in the second band, corroborating its character of a $\gamma$ band $(K=2)$ built on the second band. The comparison of obtained transition probabilities and quadrupole moments with both the experimental results and SM calculations showed a very good qualitative agreement.

Comparison between the experimental level scheme and those obtained from theoretical calculations showed that the shell-model calculations reproduce the level scheme of ${ }^{42} \mathrm{Ca}$ remarkably well, while all level energies are overestimated 
by BMF calculations. The authors of [218] explain this discrepancy by the lack of some degrees of freedom in the set of HFB wave functions used to perform the symmetry restoration and shape mixing.

The deformed-basis antisymmetrised molecular dynamics calculations with the generator coordinate method (AMD + GCM) predicts $\beta \approx 0.43$ for the band built on the $0_{2}^{+}$state of the $6 \mathrm{p}-4 \mathrm{~h}$ configuration. Furthermore, the importance of triaxiality in this system has been clearly revealed: the spectroscopic quadrupole moment of the $2_{3}^{+}$state has been found to be of similar magnitude but of opposite sign to that of the $2_{2}^{+}$state. In addition, the calculations yielded a low-lying $3^{+}$ state, which is connected by a strong transition to the $2_{3}^{+}$state and has a spectroscopic quadrupole moment close to zero. Hence, these states were identified as members of the $\operatorname{SD} \gamma$ band built on the $2_{3}^{+}$level, having a structure dominated by the $6 p-4 h$, similar to that of the $2_{2}^{+}$and $0_{2}^{+}$states.

In summary, the exceptional opportunity to reach superdeformed states via Coulomb excitation enabled a new access path and in-depth study of these elusive configurations. The results of most advanced analysis technique employing the GOSIA code and its multi-dimensional fitting procedure yielded precise and partially even model independent observables related to quadrupole deformation and triaxiality. In contrast to heavier superdeformed nuclei, the theoretical description of the new findings is achieved with a multitude of new approaches including shell-model calculations. The coexistence of a weakly deformed ground-state band and highly deformed slightly triaxial side band was established reproducing well the general picture of shape coexistence in ${ }^{42} \mathrm{Ca}$.

\subsection{High-spin structure in ${ }^{40} \mathrm{~K}$}

The region around the $\mathrm{N}=20$ and $\mathrm{Z}=20$ shell closures has been the subject of a number of experimental and theoretical investigations because not only the expected single-particle excitations, with configurations based on a spherical core are known. But also collective excitations, in particular superdeformed rotational bands, are present at double-magic ${ }^{40} \mathrm{Ca}$ [221] and the close by neighbors ${ }^{42} \mathrm{Ca}$ [217], and ${ }^{36} \mathrm{Ar}$ [222]. Rotational structures are not only present in the even-even nuclei, but regular rotational bands of unnatural parity states have also been observed in odd-A nuclei, for example, in ${ }^{43} \mathrm{Ca}$ [223], ${ }^{45} \mathrm{Sc}$ [224], as well as in odd-odd nuclei, for example, ${ }^{46} \mathrm{~V}$ [225,226].

New results have been obtained for odd-odd ${ }^{40} \mathrm{~K}$ using $\gamma \gamma$ coincidences employing just one AGATA triple cluster detector [227]. The existing knowledge of high-spin states in ${ }^{40} \mathrm{~K}$ was limited to the yrast band with $J^{\pi} \leq 9^{+}$and two negative-parity states with $J^{\pi}=\left(8^{-}, 10^{-}\right)$and $J^{\pi}=\left(9^{-}, 11^{-}\right)$, respectively.

During the AGATA demonstrator commissioning experiment, several states with excitation energy up to $8 \mathrm{MeV}$ and spin $J^{\pi} \leq 9$ have been discovered in ${ }^{40} \mathrm{~K}$. Shell-model calculations in a large model space, for neutrons and protons of two main shells, reproduce well the experimental findings. Up to six particles are allowed to move from the $s d$ to the $p f$ shell. The results of these calculations for the yrast and yrare states with $6 \leq J^{\pi} \leq 10$ are shown in Fig. 51 and compared to the extended level scheme.

The observed states are described by two multiplets. (i) the weak coupling of a proton-neutron aligned $(J=3) T=0$ pair to the $6^{+}$state in ${ }^{42} \mathrm{Ca}$ (see left side of level scheme, Fig. 51). (ii) The positive-parity yrast states with $6^{+} \leq J^{\pi} \leq 9^{+}$ are described in the shell model as two-particle and two-hole states, with mainly a $\left(d_{3 / 2}^{-2} f_{7 / 2}^{2}\right)$ configuration (see right side of level scheme, Fig. 51).

\subsection{Shape changes and the island of deformation along $N=60$ isotones}

In the region of the Segré chart with $A \approx 100$, it is well established that $\mathrm{Sr}, \mathrm{Zr}$, and Mo isotopes exhibit a large prolate deformation in the ground state. Approaching the region from the $N=50$ shell closure, where nuclei show a structure typical of the single-particle motion in a spherical potential, the sudden change between the spherical and well-deformed nuclei occurs at $N=60$. It was suggested that the sudden change in deformation is due to shape coexistence, with a spherical configuration that is the ground state and a deformed excited state below $N=60$ and that above $N=60$ these configurations are interchanged. The experimental evidence goes back to the 1970s [228-230] and more recent measurements of isotope shifts and two-neutron separation energies [231], show clear evidence for shape coexistence in the $N=60$ isotones from strontium to niobium, which vanishes in molybdenum. The neutron-rich nuclei around ${ }^{100} \mathrm{Zr}$ are remarkable for the sudden onset of deformation at $N=60$, which gives rise to an abrupt drop in the energy of the $2_{1}^{+}$state accompanied by a sharp rise in the $B\left(E 2 ; 0_{1}^{+} \rightarrow 2_{1}^{+}\right)$value for zirconium and strontium.

First theoretical descriptions of shape coexistence were given in terms of the proton-neutron interaction between the $\pi 0 g_{9 / 2}$ and $\nu 0 g_{7 / 2}$ orbitals. Filling the $\nu 0 g_{7 / 2}$ orbital makes it energetically favorable to promote protons into the $\pi 0 g_{9 / 2}$ orbital, giving rise to deformation [229,230].

The effect in $N=60$ nuclei becomes less pronounced with increasing $Z$, however the data for the lower-Z isotopes were sparse. Prior to the AGATA experiment in ${ }^{96} \mathrm{Kr}$, a mass measurement suggested that for the krypton isotopes, there was little evidence of a strong shape transition [232], but a measurement of the $2_{1}^{+}$energy in ${ }^{96} \mathrm{Kr}$ of $241 \mathrm{keV}$ seemed to contradict this conclusion [233]. However, the peak at $241 \mathrm{keV}$ in ${ }^{96} \mathrm{Kr}$ was not observed in a later Coulomb-excitation experiment performed at ISOLDE. Instead, a peak was seen at $554 \mathrm{keV}$ and it was concluded that this peak corresponds to the $2_{1}^{+}$to ground-state transition in ${ }^{96} \mathrm{Kr}$. Moreover, a moderate $B\left(E 2 ; 0_{1}^{+} \rightarrow 2_{1}^{+}\right)$value for that transition and the small quadrupole moment of the $2_{1}^{+}$state were consistent with very weak deformation for ${ }^{96} \mathrm{Kr}$ [234]. 


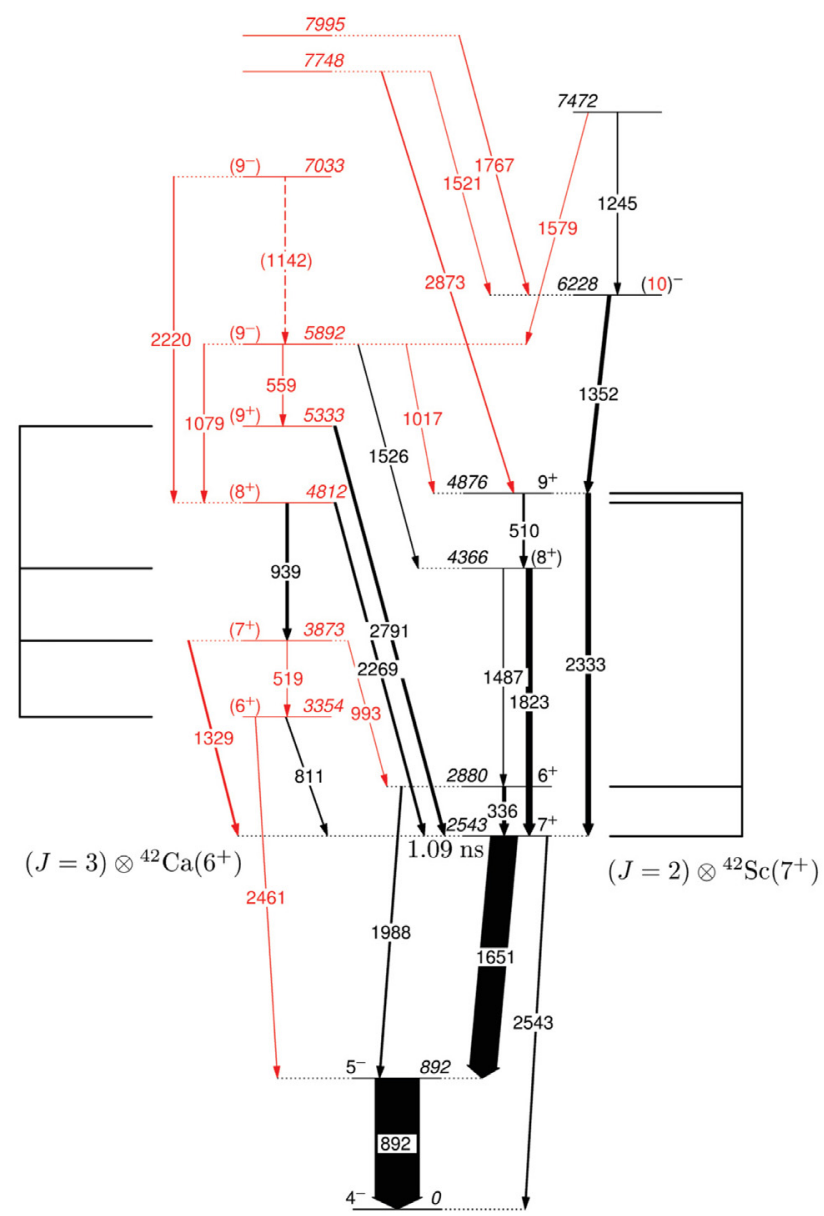

$10^{-} \quad 8057$

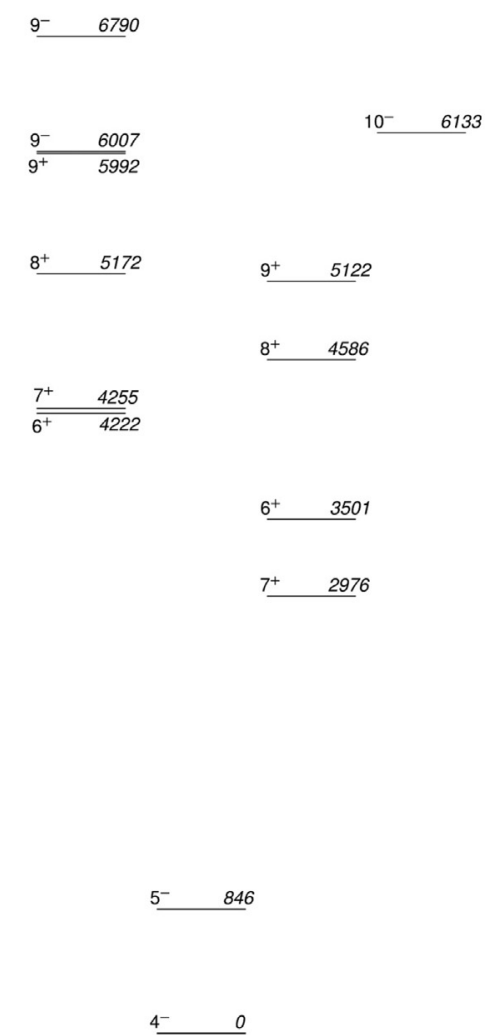

Fig. 51. Extended level scheme of ${ }^{40} \mathrm{~K}$ as obtained in the AGATA experiment by [227] and level scheme obtained from shell-model calculations (right). New information from AGATA is indicated in red. The width of the arrows corresponds to the intensity of the $\gamma$ rays. For the positive-parity states, the assigned configurations in the weak-coupling basis are also shown. Source: Figure taken from [227].

The AGATA experiment on ${ }^{96} \mathrm{Kr}$ confirmed the higher $554 \mathrm{keV}$ excitation energy of the $2_{1}^{+}$state, and it extended the level scheme by detection of the $4_{1}^{+}$state [235]. The nucleus ${ }^{96} \mathrm{Kr}$ was populated in transfer- and fusion-induced fission reactions. The measurement was performed at GANIL using an intense ${ }^{238} \mathrm{U}$ beam impinging on a thin ${ }^{9} \mathrm{Be}$ target. Due to the inverse kinematics the fission fragments were forward focused at high velocities, resulting in both efficient detection and isotopic identification of ${ }^{96} \mathrm{Kr}$ in the VAMOS++ spectrometer.

The authors of [235] provided a systematic characterization of the evolution of the nuclear structure along isotopic chains which included a systematic study of the energies of the first excited states $\mathrm{E}\left(2_{1}^{+}\right)$and $\mathrm{E}\left(4_{1}^{+}\right)$, the ratio $\mathrm{R}_{4 / 2}=\mathrm{E}\left(4^{+}\right) / \mathrm{E}\left(2^{+}\right)$and the corresponding reduced transition probabilities $\mathrm{B}(\mathrm{E} 2)$. The new experimental information is shown together with the available values along the isotopic chains of even-even nuclei with $50 \leq N \leq 64$ and $36 \leq Z \leq 42$ in Fig. 52. A clear correlation between $\mathrm{R}_{4 / 2}$ and $B\left(E 2 ; 0_{1}^{+} \rightarrow 2_{1}^{+}\right) / A$ is observed and the smooth increase of $\mathrm{R}_{4 / 2}$ as a function of the neutron number illustrates the evolution from spherical $(\approx 1.5)$ to well-deformed $(\approx 3)$ nuclear shapes. Similarly, the corresponding B(E2) continuously increases, indicating a larger collectivity. For Sr and Zr, the trend is relatively flat for $N \leq 58$, while a sudden increase is observed at $N=60$, and both indicators remain well correlated. In contrast, for $\mathrm{Kr}, \mathrm{R}_{4 / 2}$ increases more steeply than $B\left(E 2 ; 0_{1}^{+} \rightarrow 2_{1}^{+}\right) / A$ for $N \leq 54$ and decreases beyond $N=54$, while $B\left(E 2 ; 0_{1}^{+} \rightarrow 2_{1}^{+}\right) / A$ continues increasing gradually. The newly obtained $\mathrm{R}_{4 / 2}$ ratio reported by [235] shows that $\mathrm{R}_{4 / 2}$ continuously decreases till $N=60$ as shown in Fig. 52 extending the opposite trends of $\mathrm{R}_{4 / 2}$ and $B\left(E 2 ; 0_{1}^{+} \rightarrow 2_{1}^{+}\right) / A$ up to ${ }^{96} \mathrm{Kr}$.

The different behavior of neutron-rich $\mathrm{Kr}$ isotopes with respect to the neighboring isotopic chains and their sudden transition observed for $\mathrm{Sr}, \mathrm{Zr}$, and $\mathrm{Mo}$ at $\mathrm{N}=60$ was confronted with different theoretical approaches for the ${ }^{96} \mathrm{Kr}$ nucleus [234,237-239]. The right panel in Fig. 52 presents the calculated energies, using different beyond mean-field approaches, in ${ }^{96} \mathrm{Kr}$. For this nucleus, values of Delaroche et al. [239] show the best agreement with the experimental data. 


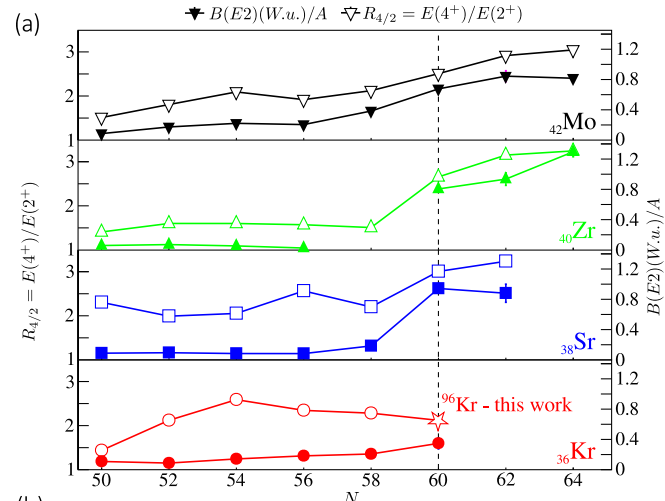

(b)

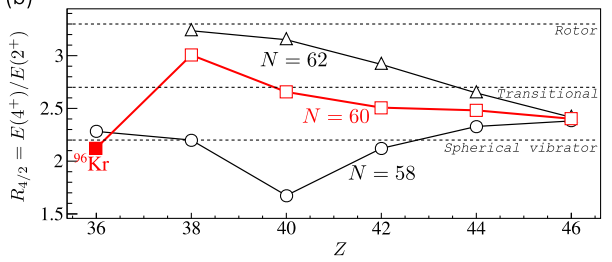

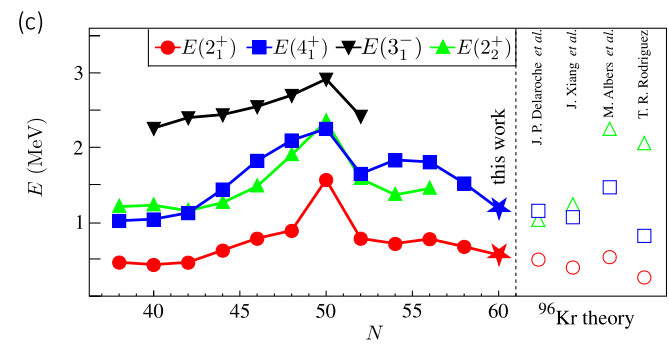

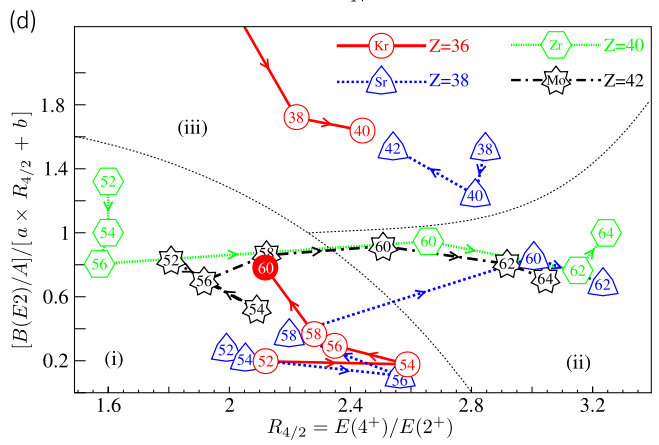

Fig. 52. Left panel: (a) The ratio $\mathrm{R}_{4 / 2}=\mathrm{E}\left(4^{+}\right) / \mathrm{E}\left(2^{+}\right)$(open symbols, left axis) of the energies of the first excited $4_{1}^{+}$and $2_{1}^{+}$states and reduced transition probabilities expressed in Weisskopf units (solid symbols, right axis) in even-even nuclei as a function of the neutron number. (b) The $\mathrm{R}_{4 / 2}$ ratio against the proton number $\mathrm{Z}$ for $\mathrm{N}=58-62$ isotonic chains. Horizontal dashed lines represent a schematic classification between spherical-vibrational and rotor nuclei. Figures adapted from Ref. [235]. Right panel: (c) Systematic evolution of $2_{1}^{+}, 4_{1}^{+}, 3_{1}^{-}$, and $2_{2}^{+}$, states for the Kr isotopic chain. On the right-hand side are reported the latest theoretical mean-field calculations for the ${ }^{96} \mathrm{Kr}$ nucleus. (d) Measured [B(E2)/A]/[a $\left.\times \mathrm{R}_{4 / 2}+\mathrm{b}\right] \mathrm{values}$ against $\mathrm{R}_{4 / 2}$ with B(E2) expressed in Weisskopf units, for isotopes of Mo, Zr, Sr, and Kr (where a=0.756 and b= -1.156 ). Regions: (i) spherical vibrator, (ii) rotor, and (iii) prolate-oblate shape coexistence with strong mixing, separated by dotted black lines, are also indicated. The expected value for a spherical vibrator is taken from [236]. ${ }^{72} \mathrm{Kr}$ is out of scale at coordinates $(1.86,2.89)$. The neutron number is indicated inside the symbols. Figures adapted from [235].

The AGATA results highlight an abrupt transition in collectivity as a function of the proton number at $\mathrm{Z}=36$, which is similar to that observed at $\mathrm{N}=60$ at higher $\mathrm{Z}$ values. It was concluded by [235] that a possible reason for this abrupt transition could be related to the insufficient proton excitations in the $g_{9 / 2}, d_{5 / 2}$, and $s_{1 / 2}$ orbitals to generate strong quadrupole correlations or to the coexistence of competing different shapes. The unexpected continuous decrease of $\mathrm{R}_{4 / 2}$ as a function of the neutron number up to $N=60$ establishes the Kr-isotopic chain as the low-Z boundary of the island of deformation for $\mathrm{N}=60$ isotones.

\subsection{North-west of ${ }^{132} \mathrm{Sn}:$ Nuclear structure in the $50 \leq Z, N \leq 82$ region}

Comprehensive studies of nuclei in the proximity of doubly-magic nuclei are of fundamental interest providing essential information on key ingredients of modern shell-model interactions like effective charges, single-particle energies, two-body matrix elements, and the tensor part of the residual interaction, which is the most difficult part to reproduce. Moreover, experimental data is needed to benchmark theoretical predictions in order to deduce improved parameters or phenomenological corrections to nuclear shell-model calculations. Detailed spectroscopic information is available up to the highest spins and excitation energies in nuclei around readily accessible closed-shell nuclei like the stable ${ }^{48} \mathrm{Ca}$ and partially ${ }^{208} \mathrm{~Pb}$. Otherwise, information is limited adjacent to exotic neutron- or proton-rich doubly-magic nuclei like $48,78 \mathrm{Ni}$ and ${ }^{100,132} \mathrm{Sn}$.

Intermediate and high-spin features of neutron-rich nuclei in the vicinity of ${ }^{132} \mathrm{Sn}$ were studied preferentially via prompt $\gamma$-ray spectroscopy of fission fragments which are populated by spontaneous fission sources, thermal neutroninduced fission of actinides or the exploitation of fusion-fission following fusion-evaporation reactions. Experimental campaigns were undertaken employing ${ }^{248} \mathrm{Cm}$ and ${ }^{252} \mathrm{Cf}$ fission sources at the GAMMASPHERE array, the EUROBALL setup at the Vivitron accelerator of IReS (Strasbourg) and the Tandem XTU accelerator in Legnaro or the EUROGAM array at ILL. By exploiting triple- and higher-fold $\gamma$-coincidence events, the data enabled a plethora of new discoveries and have given great insight into the structure of very neutron-rich systems.

However, nuclei such as ${ }^{136} \mathrm{Ba}$ or ${ }^{137} \mathrm{Ba}$ are not accessible with sufficient yields from spontaneous fission. Another gateway to neutron-rich systems is fusion-fission after fusion evaporation reactions. For instance, various reaction products of ${ }^{12} \mathrm{C}+{ }^{238} \mathrm{U}$ and ${ }^{18} \mathrm{O}+{ }^{208} \mathrm{~Pb}$ reactions were studied utilizing the EUROBALL spectrometer at the Legnaro XTU Tandem accelerator and at the Strasbourg Vivitron accelerator complex. Comprehensive results on high-spin structures were obtained for several nuclei in the $50 \leq Z, N \leq 82$ region, such as for $N=82$ nuclei ranging from ${ }^{136}$ Xe to ${ }^{140} \mathrm{Ce}$, for 


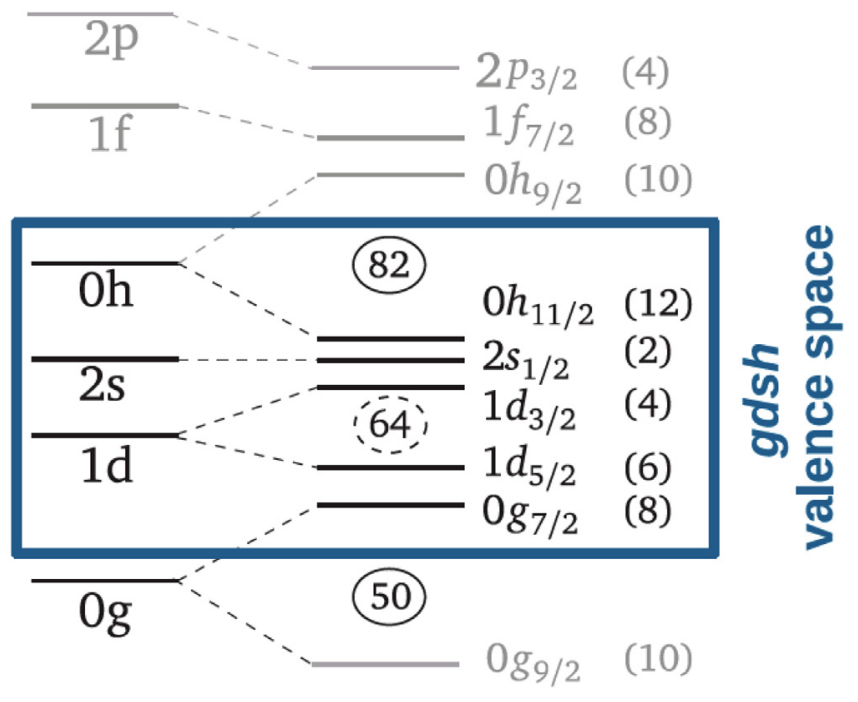

Fig. 53. Single-particle orbitals in the nuclear shell model, arranged by Brown et al. [250]. Orbitals within the $0 g 7 / 2,1 d 5 / 2,1 d 3 / 2,2 s 1 / 2$, and $0 h 11 / 2$ ( $g d s h$ ) valence space are located between the 50 and 82 magic numbers.

the odd-odd $N=81$ isotones ${ }^{136} \mathrm{Cs}$ and ${ }^{138} \mathrm{La}$, and for the ${ }^{119-126} \mathrm{Sn}$ and ${ }^{124-131} \mathrm{Te}$ chains. These experiments require very selective coincidences to identify specific reaction channels from hundreds of populated channels.

Multinucleon transfer reactions induced by heavy ions offer an efficient gateway for the synthesis of neutronrich nuclei along the valley of stability and towards the neutron-rich side that cannot be produced by means of aforementioned production methods. Highly selective experiments with thin targets become possible by combining the $\gamma$ spectrometer AGATA with high-resolution and large-acceptance mass spectrometers with trajectory reconstruction. Particle- $\gamma$ coincidences enable a significant increase of the detection limits. Most important, $\gamma$-ray transitions are uniquely attributed to the specific isotopes by identifying both mass and charge. A very precise Doppler correction for $\gamma$ rays emitted by both beam- and target-like fragments is achieved by the position sensitive $\gamma$-ray tracking device.

Several multinucleon transfer experiments were carried out at the AGATA+PRISMA setup at LNL (Italy), as well as at the VAMOS+EXOGAM and VAMOS+AGATA setups at GANIL(France). During the physics campaign at the LNL from 2010 to 2011, the AGATA spectrometer in the demonstrator configuration was installed around the target position. The magnetic mass spectrometer PRISMA enabled identification of mass $\mathrm{A}$, nuclear charge $\mathrm{Z}$ and atomic charge state $\mathrm{q}$ of the incoming ions. This detector combination is especially suited for the spectroscopy of exotic nuclei populated in multinucleon transfer reactions since the selectivity of the reaction channel in a mass spectrometer is superior to that of $\gamma \gamma-$ or $\gamma \gamma \gamma$ coincidences in thick-target experiments if no $\gamma$-ray transitions are known for the nucleus of interest. Noticeable cases from the recent past are the challenging $\gamma$-ray spectroscopy study of neutron-rich ${ }^{196} \mathrm{Os}$, in the $-2 \mathrm{p}$ channel after ${ }^{82} \mathrm{Se}^{198} \mathrm{Pt}$ multinucleon transfer [240] and the yrast-band spectroscopy of ${ }^{168,170} \mathrm{Dy}$ in the ${ }^{82} \mathrm{Se}+{ }^{170} \mathrm{Er}$ reaction [241].

\subsection{The gdsh valence space and isomeric states in the $50 \leq Z, N \leq 82$ region}

Few-valence-particle nuclei in the vicinity of doubly-magic ${ }^{132} \mathrm{Sn}$ are expected to show a single-particle structure within the gdsh valence space which is generated by the major shells $0 g_{7 / 2}, 1 d_{5 / 2}, 0 d_{3 / 2}, 2 s_{1 / 2}$, and $0 h_{11 / 2}$ [242]. The orbital arrangement including single-particle orbitals above and below the gdsh valence space is shown in Fig. 53 . The gdsh valence space is embedded between the shell closures at $Z, N=50$ and 82 magic numbers and it is dominated by the high- $j$ intruder $0 h_{11 / 2}$ orbital with its large single-particle angular momentum. The energy gap between the proton single-particle energies of the $1 d_{5 / 2}$ and $1 d_{3 / 2}$ orbits indicates a subshell closure at $Z=64$.

The nuclear structure of xenon isotopes attracted renewed attention in the field of dark-matter physics. For example, the currently running XENON100 [243] and future XENON1T [244] experiments (located at the Laboratori Nazionali del Gran Sasso (LNGS) in Italy) are based on liquid xenon as both target and detection material in the search for weakly interacting massive particles (WIMPS). The active detector material in this dark-matter experiment consists mainly of the xenon isotopes ${ }^{129} \mathrm{Xe}$ and ${ }^{131} \mathrm{Xe}$, which have high natural isotopic abundances ( $26.4 \%$ and $21.2 \%$, respectively). These isotopes exhibit a non-zero ground-state spin and spin-dependent cross sections of inelastic and elastic dark-matter scattering are under discussion. Detailed nuclear shell-model calculations are ongoing to calculate structure functions describing the spin content of the proton and neutron groups within the nucleus [245]. Several shell-model studies concentrated on this aspect of WIMP-nucleon coupling involving e.g. ${ }^{131} \mathrm{Xe}$ [246-249].

Excitations in nuclei near to ${ }^{132} \mathrm{Sn}$ are expected to be of predominantly single-particle character. Metastable isomeric states are caused by different mixtures of $g d s h$ orbitals and indicate large configuration differences and a reduced overlap 


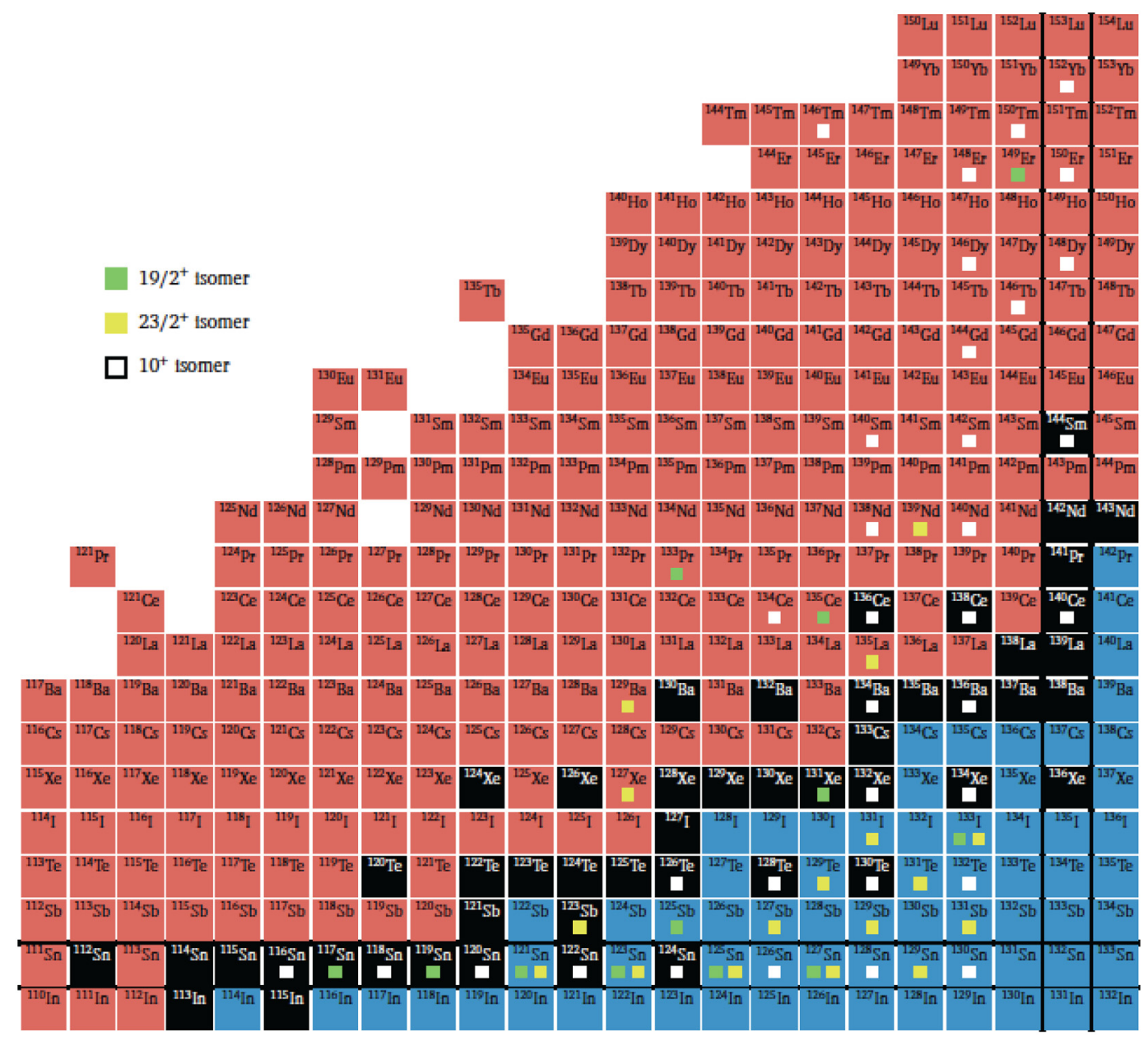

Fig. 54. Partial chart of nuclei containing all hitherto known $J^{\pi}=19 / 2^{+}$(green boxes), $J^{\pi}=23 / 2^{+}$(yellow boxes), and $J^{\pi}=10^{+}$(white boxes) isomers with $T_{1 / 2}>10 \mathrm{~ns}$.

Source: Data compiled from the Atlas of Nuclear Isomers [251] and Refs. [252,253].

between initial and final wave functions. Thus, isomers are related to an abrupt change of the nuclear configuration and open a gateway for a detailed understanding of the underlying structure [254]. Hence, the evolution of isomers and states built on top as a function of the gradual filling of shell-model orbitals along shell closures are of particular interest. Following isomer mechanisms are established in the mass region. Spin traps with large changes in spin require higher-order multipole decays which are accompanied by lower transition rates and longer lifetimes. Small transition energies between states cause longer lifetimes as the electromagnetic transition rate depends on $E_{\gamma}^{(2 \lambda+1)}$ favoring highenergy transitions and the lowest possible multipolarity. Latter cases are often related to seniority isomers in the $50 \leq$ $\mathrm{Z}, \mathrm{N} \leq 82$ region.

The following series of AGATA publications from the LNL campaign elucidated isomerism in this mass region : 'Highspin structure of ${ }^{134} \mathrm{Xe}$ ' [255], 'Isomers and high-spin structures in the $N=81$ isotones ${ }^{135} \mathrm{Xe}$ and ${ }^{137} \mathrm{Ba}$ ' [256], 'High-spin structures in ${ }^{132} \mathrm{Xe}$ and ${ }^{133} \mathrm{Xe}$ and evidence for isomers along the $N=79$ isotones', [257], 'High-spin structure in the transitional nucleus ${ }^{131} \mathrm{Xe}$ : Competitive neutron and proton alignment in the vicinity of the $N=82$ shell closure' [258], 'Millisecond $23 / 2^{+}$isomers in the $N=79$ isotones ${ }^{133} \mathrm{Xe}$ and ${ }^{135} \mathrm{Ba}$ ' [259], 'Isomer spectroscopy in ${ }^{133} \mathrm{Ba}$ and high-spin structure of ${ }^{134} \mathrm{Ba}$ ' [260], and 'Identification of high-spin proton configurations in ${ }^{136} \mathrm{Ba}$ and ${ }^{137} \mathrm{Ba}$ ' [261].

The intruder $0 h_{11 / 2}$ neutron orbital allows high- $j$ couplings and it is responsible for several characteristic yrast spingap isomers. Fig. 54 shows the distribution of all hitherto known $J^{\pi}=10^{+}, 19 / 2^{+}$and $23 / 2^{+}$isomers with half-lives longer than $10 \mathrm{~ns}$ in the $50 \leq Z, N \leq 82$ range. The negative magnetic moments of the $J^{\pi}=10^{+}$states in nuclei below the $Z=64$ subshell closure corroborate a near-spherical, maximally aligned two-neutron-hole $v h_{11 / 2}^{-2}$ configuration [270-272]. Hence, the wide spread of the $J^{\pi}=10^{+}$isomers throughout this region emphasizes the importance of the intruder $v h_{11 / 2}$ orbital. Half-life values extend from lower nanosecond values up to $198(3) \mathrm{ms}$ in ${ }^{146} \mathrm{Tm}$ [273]. Very small energy differences of the $10^{+} \rightarrow 8^{+}$transition were observed in ${ }^{130} \mathrm{Te}(18 \mathrm{keV})[274]$ and ${ }^{134} \mathrm{Xe}(28 \mathrm{keV})$ [275]. Noteworthy 

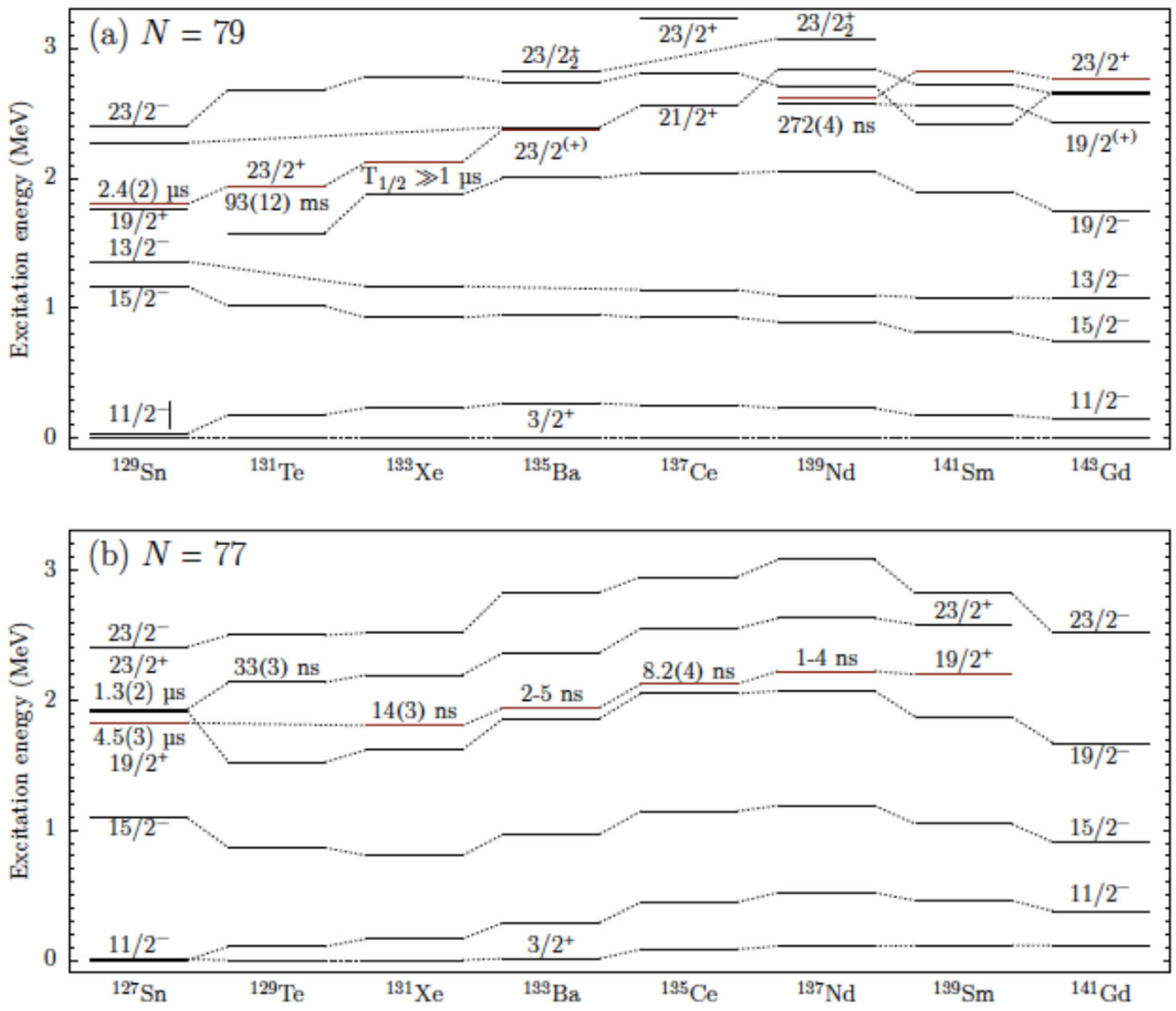

Fig. 55. Evolution of excited states along the (a) $N=79$ and (b) $N=77$ chains. Corresponding $T_{1 / 2}$ values are given in the figure. Isomeric $J^{\pi}=23 / 2^{+}$states and candidates for this isomer in ${ }^{133} \mathrm{Xe}$ and ${ }^{135} \mathrm{Ba}$ are highlighted with thick red lines. Isomeric $J^{\pi}=19 / 2^{+}$states along $N=77$ isotones are indicated with thick red lines.

Source: Data extracted from ENSDF [253], Refs. [257,262-264] for the $N=79$ chain, and Refs. [265-269] for the $N=77$ chain.

is the long half-life of $8.39(11) \mathrm{ms}[276]$ for the $J^{\pi}=10^{+}$isomer in ${ }^{132} \mathrm{Xe}$, decaying via an $E 3$ transition to the $J^{\pi}=7^{-}$ state.

Approaching the $Z=64$ subshell closure, a reduced transition strength of the $10^{+} \rightarrow 8^{+}$decay is observed [272]. The distinct change of the nuclear structure of the $J^{\pi}=10^{+}$isomers can be traced back to pair excitations from the semi-magic $Z=64$ shell closure. This results in an increasing contribution of the proton $\pi h_{11 / 2}^{2}$ configuration to the overall angular momentum of the $J^{\pi}=10^{+}$isomers [277]. Microsecond isomers of spin $J^{\pi}=19 / 2^{+}$were observed in ${ }^{119-129} \mathrm{Sn}$ isotopes. Here, the dominant single-particle configuration is $h_{11 / 2}^{-1} \otimes 5^{-}$. Yrast isomers with spin $J^{\pi}=23 / 2^{+}$ and $J^{\pi}=27 / 2^{-}$are present along the Sn chain, too.

Similar to the $J^{\pi}=10^{+}$isomers in even-even nuclei, $J^{\pi}=19 / 2^{+}$and $23 / 2^{+}$isomers are characteristic features in even-odd nuclei. The isomers predominantly show up along the $\mathrm{Sn}, \mathrm{Sb}$ and Te isotopic chains and are less abundant in $Z \geq 54$ nuclei. Half-life values are distributed throughout the nano-, micro-, and millisecond range [263,278]. Fig. 55(a) shows the evolution of negative- and positive-parity states along the $N=79$ chain. Isomeric $J^{\pi}=23 / 2^{+}$states were systematically reported for this chain in ${ }^{129} \mathrm{Sn}$ [262], ${ }^{131} \mathrm{Te}$ [263], and ${ }^{139} \mathrm{Nd}$ [264]. These isomers are members of the seniority $v=3$ multiplet and are interpreted to have a predominant $v h_{11 / 2}^{-2} d_{3 / 2}^{-1}$ configuration. Typically, these isomers decay into first or second excited $J^{\pi}=19 / 2^{-}$states. The energy difference between initial $J^{\pi}=23 / 2^{+}$and final $J^{\pi}=19 / 2^{-}$states remains relatively constant with respect to the proton number. However, the half-life values differ by five orders of magnitude between ${ }^{131} \mathrm{Te}$ and ${ }^{139} \mathrm{Nd}$.

Candidates for isomeric counterparts in ${ }^{133} \mathrm{Xe}$ and ${ }^{135} \mathrm{Ba}$ were anticipated. First indication for a long-lived state at 2107-keV excitation energy in ${ }^{133} \mathrm{Xe}$ was found and identified as a promising candidate for the $J^{\pi}=23 / 2^{+}$isomer [257] Moreover, the analysis of the AGATA ${ }^{136} \mathrm{Xe}+{ }^{208} \mathrm{~Pb}$ MNT experiment indicated an isomeric $J^{\pi}=23 / 2^{+}$counterpart at 2388 $\mathrm{keV}$ excitation energy in ${ }^{135} \mathrm{Ba}$ [257]. The AGATA publication contributes to the systematic investigation of $J^{\pi}=23 / 2^{+}$ isomers along the $N=79$ isotones. A complementary experiment at the FN tandem accelerator at Cologne was performed with a pulsed ${ }^{9} \mathrm{Be}$ beam impinging on a ${ }^{130} \mathrm{Te}$ target. Exploiting $\gamma$-time correlations with respect to the beam pulse, halflives of 8.64(13) $\mathrm{ms}$ in ${ }^{133} \mathrm{Xe}$ and $1.06(4) \mathrm{ms}$ in ${ }^{135} \mathrm{Ba}$ were identified. The combined results of off-beam angular-correlation and internal-conversion coefficient measurements for transitions depopulating the isomers confirmed unambiguously the 
spin/parity assignments of the $2107-$ and $2388-\mathrm{keV}$ states in ${ }^{133} \mathrm{Xe}$ and ${ }^{135} \mathrm{Ba}$, respectively. The small multipole-mixing ratios indicate a dominant $M 2$ character of the 231- and 254-keV transitions. The experimental findings close a gap of $J^{\pi}=23 / 2^{+}$isomers along the $N=79$ isotones. Experimentally determined $B(M 2)$ and $B(E 3)$ transition strengths were compared to shell-model calculations employing the realistic SM, GCN50:82, SN100PN, and SN100-KTH interactions. In particular, calculated $B(M 2)$ values agree with the measured transition strengths; the systematic evolution from ${ }^{129} \mathrm{Sn}$ to ${ }^{135} \mathrm{Ba}$ is reproduced. A detailed inspection of initial and final shell-model configurations indicate a dominant $v\left(h_{11 / 2}^{-2} d_{3 / 2}^{-1}\right)$ configuration for the $J^{\pi}=23 / 2^{+}$states and a dominant $v\left(h_{11 / 2}^{-1} \pi g_{7 / 2}^{2}\right)$ configuration for the $J^{\pi}=19 / 2^{-}$states. The isomeric character of the $J^{\pi}=23 / 2^{+}$states can be traced back to the change of the neutron-configuration content and the required neutron-spin transfer between initial and final states.

$J^{\pi}=23 / 2^{+}$isomers were also discovered in the $N=77$ chain in ${ }^{127} \mathrm{Sn}\left(T_{1 / 2}=1.26(15) \mu \mathrm{s}\right.$ [279] $)$ and in ${ }^{129} \mathrm{Te}$ $\left(T_{1 / 2}=33(3)\right.$ ns [280]). From ${ }^{131}$ Xe onwards, isomeric $J^{\pi}=19 / 2^{+}$states, decaying via $19 / 2^{+} \rightarrow 19 / 2^{-} E 1$ transitions, prevail along the $N=77$ chain. Precise half-lives were evaluated in ${ }^{127} \mathrm{Sn}$ [265], ${ }^{131} \mathrm{Xe}$ [266], and ${ }^{135} \mathrm{Ce}$ [267] (c.f. Fig. 55 (b)). For ${ }^{133} \mathrm{Ba}[269]$ and ${ }^{137} \mathrm{Nd} \mathrm{[268]}$ only estimates of the corresponding half-lives in the lower nanosecond regime are known. $J^{\pi}=19 / 2^{+}$isomers in odd-mass isotopes have a dominating single-particle configuration of $v h_{11 / 2}^{-2} s_{1 / 2}^{-1}$ and can be interpreted within the particle-plus-rotor model as a coupling of a neutron (hole) to the $J^{\pi}=5^{-}$isomer of the neighboring even-mass partners $\left(v h_{11 / 2}^{-1} \otimes 5_{1}^{-}\right)[265,268,269]$.

The AGATA study 'Isomer spectroscopy in ${ }^{133} \mathrm{Ba}$ and high-spin structure of ${ }^{134}{ }^{\mathrm{Ba}}$ [260] resolved contradictory experimental findings in both nuclei. Excited states in ${ }^{133,134} \mathrm{Ba}$ were populated in the AGATA ${ }^{136} \mathrm{Xe}+{ }^{208} \mathrm{~Pb}$ MNT reaction and the ${ }^{13} \mathrm{C}+{ }^{124} \mathrm{Sn}$ fusion-evaporation experiment at the HORUS spectrometer at University of Cologne, equipped with eight high-purity germanium and twelve cerium-doped lanthanum-bromide detectors, providing detailed $\gamma \gamma$ and $\gamma \gamma$ time correlations. The evaluated half-life of the $1942-\mathrm{keV}$ state in ${ }^{133} \mathrm{Ba}$ was revised to $T_{1 / 2}=66.6(20)$ ns. Likewise, the half-life of the $2222-\mathrm{keV}\left(J^{\pi}=19 / 2^{+}\right)$state in ${ }^{137} \mathrm{Nd}$ was measured to be $0.38(7) \mathrm{ns}$, utilizing a ${ }^{16} \mathrm{O}+{ }^{125} \mathrm{Te}$ electronic fast-timing experiment. Both results close gaps of $J^{\pi}=19 / 2^{+}$isomers for $N=77$ isotones (see Fig. 55). Since $B(E 1)$ values cannot be evaluated in the $g d s h$ valence space, the $B(E 1)$ transition strength of the $19 / 2^{+} \rightarrow 19 / 2^{-}$decay in ${ }^{133} \mathrm{Ba}$ was primarily investigated by means of a cross-shell calculation in an enlarged $g d s h+v f_{7 / 2}$ valence space by the EPQQM interaction. The discussion was completed by a theoretical E2 map, calculated with the novel SNV interaction.

Several isomeric $11 / 2^{-}$, and $J^{\pi}=6^{+}, 7^{-}$states and their level systematics are also known in the region. The broad presence of $11 / 2^{-}$isomers in the odd-mass nuclei with $64<N<82$ and $50 \leq Z<64$ demonstrates the essential role of the high-j, $0 h_{11 / 2}$ intruder orbital. High-spin isomerism has been well established in the $N=81$ isotones ranging from $Z=50(\mathrm{Sn})$ to $Z=64(\mathrm{Gd})$, where the neutron $h_{11 / 2}$ orbital plays a crucial role. In ${ }^{137} \mathrm{Ba}$, spins and parities of the ground state and the $11 / 2$ isomeric state at $661.659(3) \mathrm{keV}$ were already well established. The $11 / 2^{-} \rightarrow 3 / 2^{+} \mathrm{M} 4 \gamma$-ray transition is even one of the best known energy calibration standards. Prior to the AGATA experiments, existing data on the states above the isomer were rather scarce. Moreover, important experimental details on higher-spin isomers were missing in the $N=81$ isotone ${ }^{135} \mathrm{Xe}$, i.e. the lifetime of the purported $19 / 2^{-}$isomer in ${ }^{135} \mathrm{Xe}$ was still unknown. AGATA results from [256] extended significantly the high-spin regimes of ${ }^{135} \mathrm{Xe}$ and ${ }^{137} \mathrm{Ba}$ to higher excitation energies. A new 9.0(9)-ns isomer was identified in ${ }^{135} \mathrm{Xe}$, finally closing a gap in the systematics along the $N=81$ isotonic chain.

The experimental findings were compared to results of large-scale shell-model theory considering both neutrons and protons in the full gdsh model space. The calculations corroborated most of the energies of the Xe- and Ba level schemes and the suggested assignments. The novel PQM130 (Pairing+Quadrupole-Quadrupole+Multipole for the mass region 130) interaction by Teruya et al. [209] predicts the electromagnetic transition probabilities of $19 / 2^{-}$isomer decays in ${ }^{133} \mathrm{Te}$, ${ }^{135} \mathrm{Xe}$, and ${ }^{137} \mathrm{Ba}$ with a remarkable accuracy. However, the well-established SN100PN interaction [281] partially fails in reproducing the experimental $B\left(E 2 ; 19 / 2^{-} \rightarrow 15 / 2^{-}\right)$values for ${ }^{135} \mathrm{Xe}$ and ${ }^{137} \mathrm{Ba}$. A comparison with the structure of ${ }^{133} \mathrm{Te}$ guides the interpretation of the more complex high-spin structures in ${ }^{135} \mathrm{Xe}$ and ${ }^{137} \mathrm{Ba}$ farther from double-magic ${ }^{132} \mathrm{Sn}$. The short-lived $J^{\pi}=19 / 2^{-}$high-spin isomers above the $11 / 2^{-}$states exhibit a strong proton component that is parallel to the yrast $6^{+}$isomers observed in the $N=82$ isotones. The calculated proton configurations follow the nuclear-structure features of the adjacent isotopes in the closed $N=82$ shell, validating at least the proton-proton part of the SN100PN interaction.

\subsection{High-spin features northwest of ${ }^{132} \mathrm{Sn}$}

Away from the $\mathrm{Z}=50$ and $\mathrm{N}=82$ shell closures, collective features begin to dominate over single-particle excitations due to increasing correlations among valence nucleons. Instead of pure shell-model configurations, the wave functions comprise different linear combinations of couplings of the unique-parity high-j orbital $0 h_{11 / 2}$ with the $2 s_{1 / 2}, 1 d_{3 / 2}, 1 d_{5 / 2}$, and $0 g_{7 / 2}$ orbitals [282,283]. A coarse indicator of structural evolution is given by the ratio between the excitation energies of first $J^{\pi}=2^{+}$and $4^{+}$states [284]. Fig. 56 shows a partial nuclei chart of even-even nuclei with $Z \geq 50$ and $N \leq 82$, whereby the color code indicates the experimentally determined $R=\left(E_{4^{+}} / E_{2^{+}}\right)$values, ranging from spherical ${ }^{132} \mathrm{Sn}$ $(R=1.09)$ to deformed rotors like ${ }^{132} \mathrm{Sm}(R=3.18)$. A gradual change between both extreme values is observed throughout a broad near vibration region $(R \approx 2)$. Moving toward midshell, the excitation spectra exhibit a clear rotational character $(E \approx J(J+1))$, while $A \approx 135$ nuclei are well described by assuming anharmonic vibrations [285].

A large number of nuclei have $R$ ratios between 2.5 and 3.0, indicating $\gamma$ instability [286-288]. The $\gamma$ softness of nuclei in this transitional region originates from the different deformation-driving properties of aligned $0 h_{11 / 2}$ protons (towards 


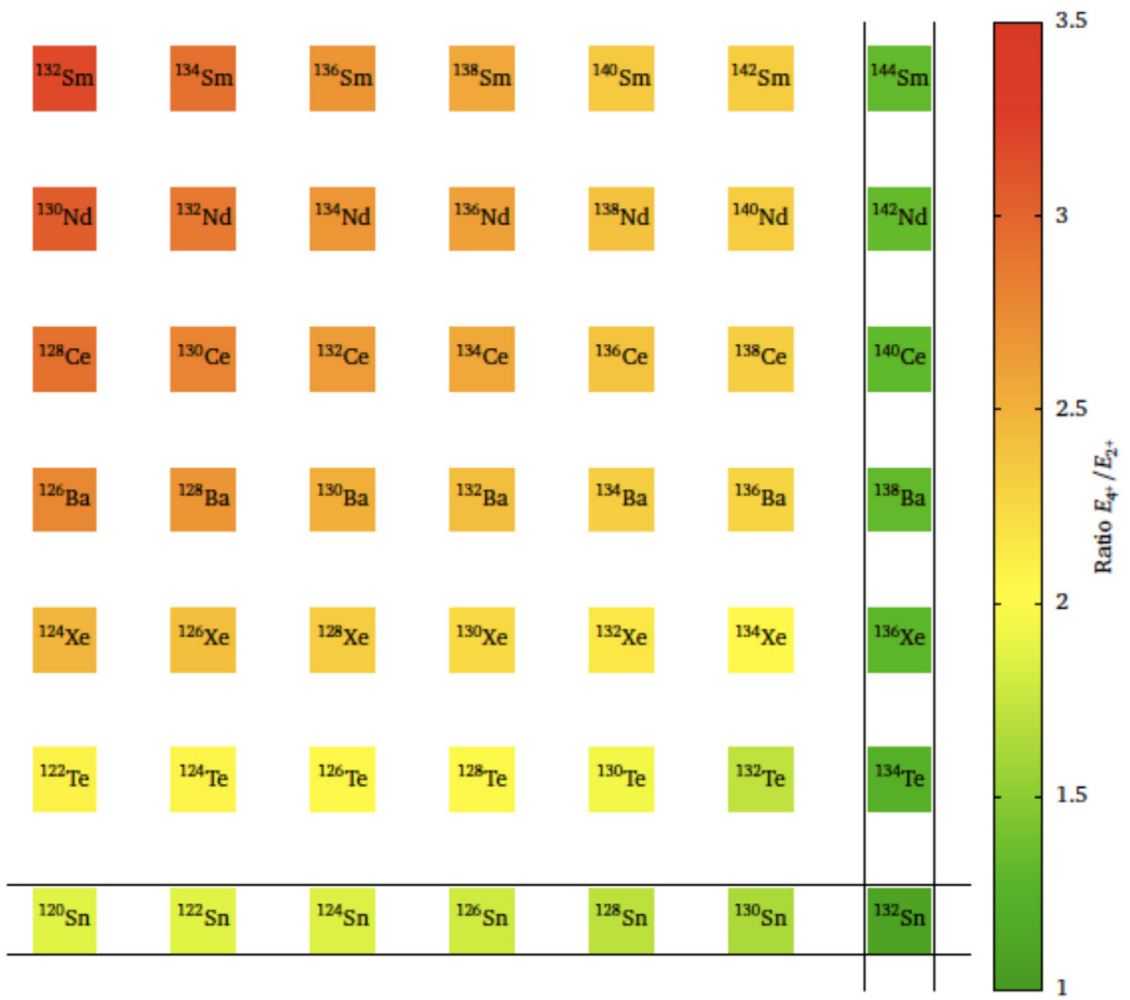

Fig. 56. $E_{4_{1}^{+}} / E_{2_{1}^{+}}$ratio in even-even nuclei northwest of ${ }^{132} \mathrm{Sn}$. Ratios range from 1.09 in ${ }^{132} \mathrm{Sn}$ to 3.18 in ${ }^{132} \mathrm{Sm}$. Solid black lines depict magic numbers.

Source: Data extracted from the NuDat 2.7 database [293].

prolate shape with $\gamma=0^{\circ}$ ) or neutrons (towards oblate shape with $\gamma=-60^{\circ}$ ). High-spin structures are particularly sensitive to mixed configurations involving the intruder $0 h_{11 / 2}$ orbital. In even-odd nuclei high-spin states are based on $J^{\pi}=11 / 2^{-}$yrast trap isomers with $v h_{11 / 2}^{-1}$ configuration. These isomers decay predominantly to the $J^{\pi}=3 / 2^{+}$ground state. Similarly, the high-spin states of even-even nuclei $(J \geq 10 \hbar)$ are build on a $J^{\pi}=10^{+}$yrast isomer with a fully spin-aligned two-quasiparticle $v h_{11 / 2}^{-2}$ configuration. In various $A \approx 130$ nuclei the following features are established: collective bands and phenomena involving the $0 h_{11 / 2}$ orbital such as shape coexistence [289], wobbling bands [290], alternating-parity bands [291], magnetic rotation in dipole bands [292].

Another high-spin feature in the mass region is a sudden increase of the total aligned angular momentum, implying a narrower level spacing between high-spin states with respect to the spacing extrapolated from the regular ground-state band [294,295]. This phenomenon is interpreted as a band crossing between the ground-state band (GS-Band) and a two-quasiparticle band ( $S$-Band), originating from the alignment of a pair of neutrons or protons occupying the intruder $0 h_{11 / 2}$ orbital. In 1972, the crossing of two bands was first observed in ${ }^{162} \mathrm{Er}$ by Johnson et al. [296]. In a semi-microscopic model, the fluidity of the nucleus reduces at the alignment frequency, resulting in a sudden increase or bending of the total aligned angular momentum over a small range of rotational frequencies [284]. The alignment process is also manifested in a sudden decrease of $B(E 2)$ values between aligning states [297]. Alignment of neutron and proton $0 h_{11 / 2}$ pairs along yrast bands were systematically reported in a series of $A \approx 125-136$ xenon, barium, and cerium isotopes. Fig. 57 presents a compilation of the total aligned angular momentum $\left(I_{x}\right)$ of the $E 2$ ground-state band for $(\mathrm{a})-(\mathrm{h}){ }^{129-136} \mathrm{Ce},(\mathrm{i})-(\mathrm{p}){ }^{127-134} \mathrm{Ba}$, and $(\mathrm{q})-(\mathrm{x}){ }^{125-132} \mathrm{Xe}$ as a function of rotational frequency $\hbar \omega$.

Backbending emerges typically at the position of the isomeric $J^{\pi}=10^{+}$state for even-mass nuclei. In ${ }^{132} \mathrm{Xe}$, the $J^{\pi}=10^{+}$isomer $\left(8.39(11)\right.$ ms [276]) decays through an $E 3$ transition to a $J^{\pi}=7^{-}$state, whereby the $J^{\pi}=8^{+}$state has not been discovered up to now. Supported by shell-model calculations, it is highly probable that the $J^{\pi}=8^{+}$state is located very close in energy to the $J^{\pi}=10^{+}$isomer, implying a remarkable strong backbending [257]. The even-mass nuclei, such as ${ }^{128,130} \mathrm{Ba}$ and ${ }^{132,134,136} \mathrm{Ce}$, exhibit the presence of two aligned $S$-bands. The first alignment refers to states above the yrast $J^{\pi}=10_{1}^{+}$state, while the second alignment pertains to states in the yrare sequence above the second $J^{\pi}=10_{2}^{+}$state. Measured $g$ factors reveal that the alignment of yrast states can be traced back to a pair of neutrons in the $0 h_{11 / 2}$ orbital, whereas the alignment of yrare states corresponds to a proton pair in the $0 h_{11 / 2}$ orbital [270]. Likewise, distinct backbendings and upbendings were discovered in various negative-parity ground-state bands of even-odd nuclei in this region. According to cranked-shell-model and total-Routhian-surface calculations, these alignments were solely 


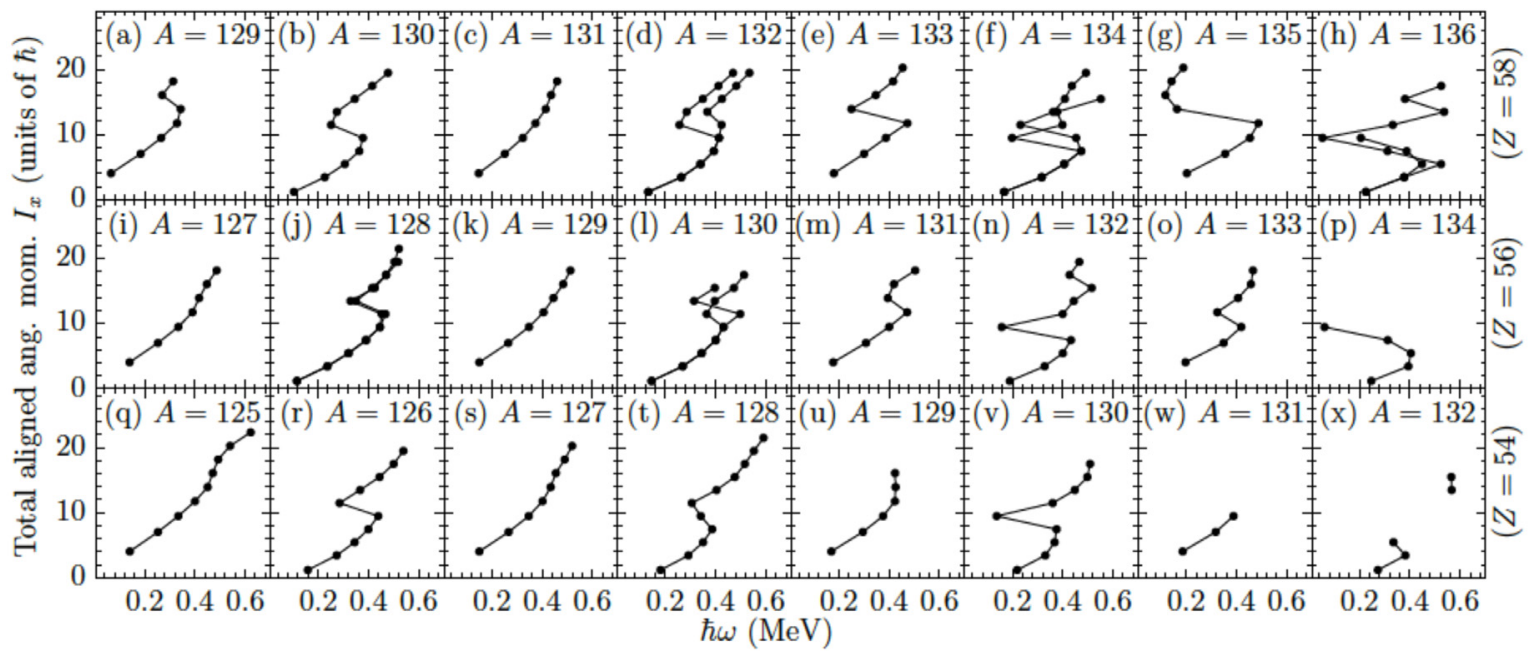

Fig. 57. Evolution of total aligned angular momentum against the rotational frequency $\hbar \omega$ for the yrast bands in $(a)-(h) C e(Z=58)$, (i) $-(p)$ Ba $(Z$ $=56)$, and $(q)-(x) X e(Z=54)$. See detail in the text.

Source: Data extracted from Refs. [253,290].

associated with the breaking of the first neutron $0 h_{11 / 2}$ pairs, except for ${ }^{129}$ Xe where an alignment of two $0 h_{11 / 2}$ protons was manifested [290]. Reliable information on alignment properties of high-spin states in ${ }^{131} \mathrm{Xe}$ and ${ }^{134} \mathrm{Ba}$ are missing within this systematic.

Newly discovered high-spin states and their properties are subject of seven AGATA studies [255-261] along two isotopic chains ${ }^{131-135} \mathrm{Xe}$ and ${ }^{133-137} \mathrm{Ba}$. While an extended irregular sequence of single-particle states with high-spin values are found in the $N=80,81$ isotones ${ }^{134,135} \mathrm{Xe}$ and ${ }^{136,137} \mathrm{Ba}$ close to the semi-magic shell closure [255,256,261], more regular rotational-like structures are found in the transitional nuclei ${ }^{131} \mathrm{Xe}$ and ${ }^{134} \mathrm{Ba}[258,260]$ (see Fig. 58).

The level scheme of ${ }^{136} \mathrm{Ba}$ above the $J^{\pi}=10^{+}$isomer was significantly extended employing prompt-delayed correlations with the GAMMASPHERE array at Lawrence Berkeley National Laboratory following a MNT reaction [272]. However, spin assignments for the new states were not given. While the high-spin scheme of the lighter isotones ${ }^{132}$ Te and ${ }^{134} \mathrm{Xe}$ are dominated by high-energy $\left(E_{\gamma}>900 \mathrm{keV}\right) 12^{+} \rightarrow 10^{+}$transitions, no reasonable candidate for the $J^{\pi}=12^{+}$ state in ${ }^{136} \mathrm{Ba}$ had been identified. As a result of the combined analysis of the AGATA ${ }^{136} \mathrm{Xe}+{ }^{238} \mathrm{U}$ MNT experiment and fusion-evaporation experiments at the HORUS spectrometer, the work in [261] revised and extended level schemes of ${ }^{136} \mathrm{Ba}$ and ${ }^{137} \mathrm{Ba}$ (see Fig. 59). Exploiting $\gamma$-ray angular distribution and $\gamma \gamma$ angular-correlation measurements, the $J^{\pi}=12^{+}$state was identified. The positive-parity yrast band between $J^{\pi}=10^{+}$and $12^{+}$states was found to be interrupted by a $J^{\pi}=11^{-}$state in ${ }^{136} \mathrm{Ba}$. Using the same technique, the $3545-\mathrm{keV}$ state in ${ }^{137} \mathrm{Ba}$ was identified as the bandhead of the positive-parity band.

The experimental findings were compared to untruncated large-scale shell-model calculations using the gdsh valence space for protons and neutrons outside doubly magic ${ }^{100} \mathrm{Sn}$, employing the shell-model code NUSHELLX@MSU [298], the massive-parallelization code KSHELL [299], and the ANTOINE shell-model code [300]. Four different shell-model interactions were used for comparison: GCN50:82 by [301,302], the realistic shell model developed by [303,304], the pair-truncated shell model PQM130 by [209,305], the SN100PN interaction [281]. As a result of the GCN50:82 interaction, the $J^{\pi}=10^{+}$isomeric state was assigned to an almost pure $\nu h_{11 / 2}^{-2}$ neutron configuration, while the $J^{\pi}=12^{+}$state was identified as a fully aligned $\pi\left(g_{7 / 2}^{4} d_{5 / 2}^{2}\right)$ proton spin configuration. The $J^{\pi}=11^{-}$state was associated with a neutron character, corroborating the interruption of the positive-parity band. A continuation via a $v h_{11 / 2}^{-2}$ configuration is energetically favorable over a direct $12^{+} \rightarrow 10^{+}$decay. Calculated $B\left(E 2 ; 12^{+} \rightarrow 10^{+}\right.$) values in ${ }^{134}$ Xe and ${ }^{136} \mathrm{Ba}$ follow the observed decay pattern in both nuclei. However, SN100PN fails short to reproduce the nuclear-structure change of positive-parity states between $Z=54$ and 56, most likely attributable to the deficient monopole part of the interaction. A comparison between calculated configurations of positive-parity states in ${ }^{136} \mathrm{Ba}$ and ${ }^{137} \mathrm{Ba}$ supports the interpretation of the $J^{\pi}=21 / 2^{+}$state in even-odd ${ }^{137} \mathrm{Ba}$ as the coupling of a valence neutron to the $J^{\pi}=12^{+}$state in even-even ${ }^{136} \mathrm{Ba}$.

Experiments employing the ${ }^{136} \mathrm{Xe}+{ }^{198} \mathrm{Pt},{ }^{136} \mathrm{Xe}+{ }^{208} \mathrm{~Pb}$ and ${ }^{136} \mathrm{Xe}^{238} \mathrm{U}$ multinucleon-transfer reactions with heavy target and projectile are favorable to populate states with higher spins and excitation energy. The ${ }^{136} \mathrm{Xe}+{ }^{238} \mathrm{U}$ AGATA experiment enabled a first time discrimination between the two different population paths, multinucleon transfer and fission. In ${ }^{134} \mathrm{Xe}$, new high-spin states were discovered on top of the two long-lived $10^{+}$and $7^{-}$isomers. The extended and revised level scheme of ${ }^{134} \mathrm{Xe}$ was constructed up to an excitation energy of $5.832 \mathrm{MeV}$ with tentative spin-parity assignments 

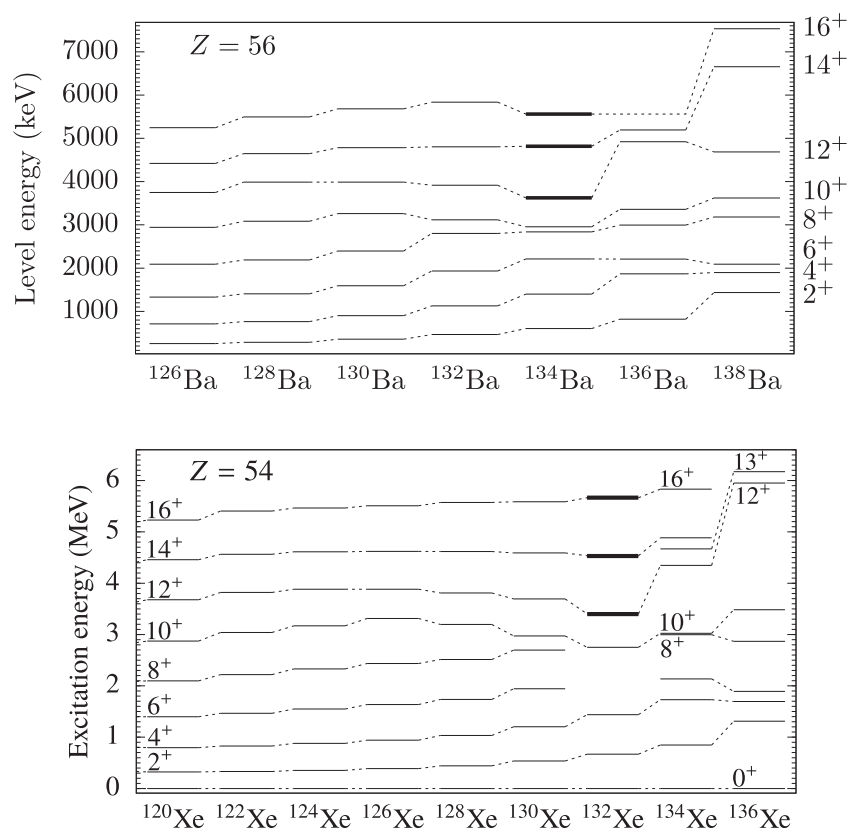

Fig. 58. Top panel: Evolution of positive-parity yrast states along the $Z=56$ even-mass Ba isotopes. Newly discovered states in ${ }^{134} B a$ are marked with thick lines. Data taken from Refs. [260,293]. Bottom panel: Evolution of positive-parity yrast states along the $Z=54$ even-mass Xe isotopes. Newly discovered states in ${ }^{132} \mathrm{Xe}$ are marked with thick lines. Data taken from Refs. [257].

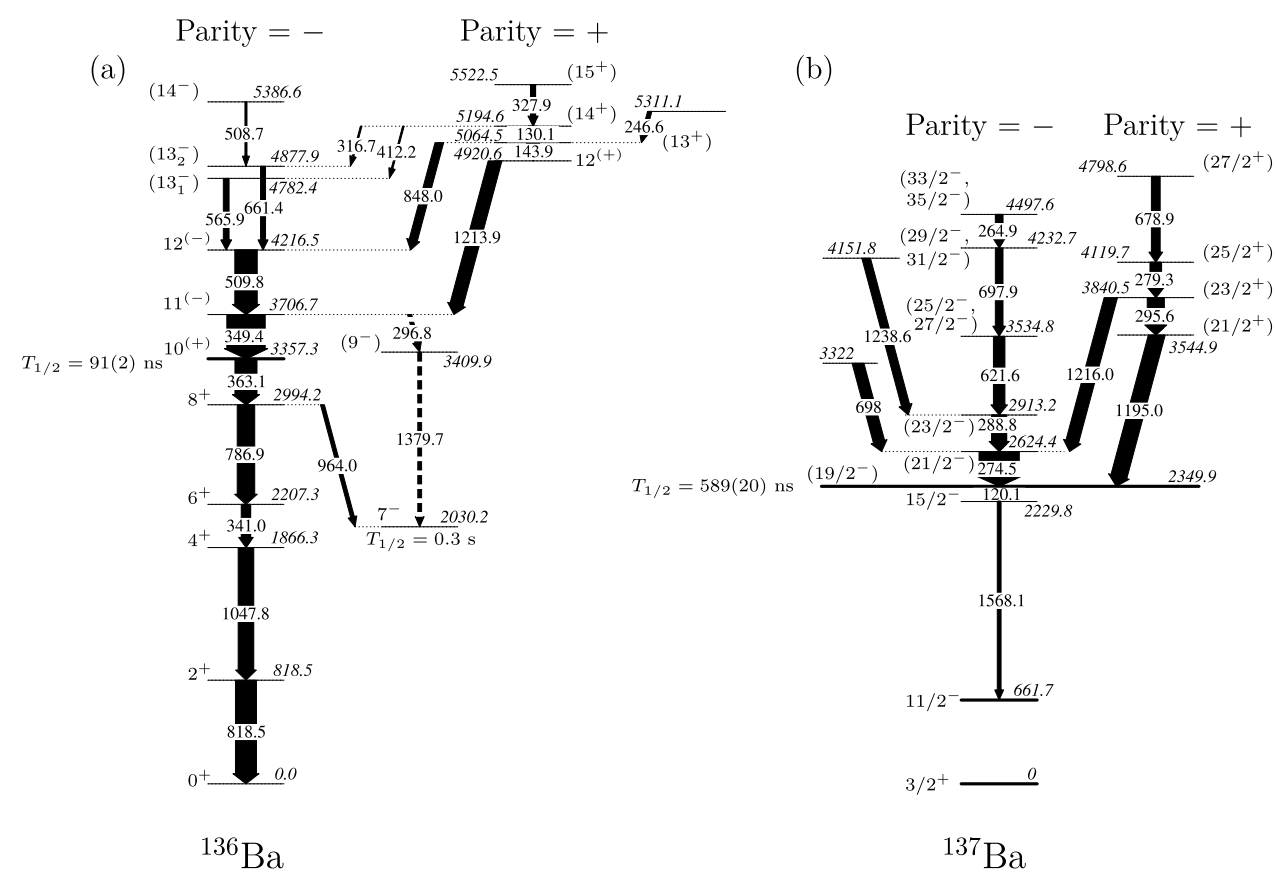

Fig. 59. Revised and extended level schemes of ${ }^{136} \mathrm{Ba}$ and ${ }^{137} \mathrm{Ba}$ were obtained from the combined analysis of the AGATA ${ }^{136} \mathrm{Xe}+{ }^{238} \mathrm{U}$ MNT experiment and fusion-evaporation experiments.

Source: Figure taken from Refs. [261].

up to $16^{+}$. Previous assignments of several states above the $7^{-}$isomer have been revised by introducing two newly observed $\gamma$ rays in two decay branches. The high-spin structure above the $10^{+}$isomer was identified unambiguously 

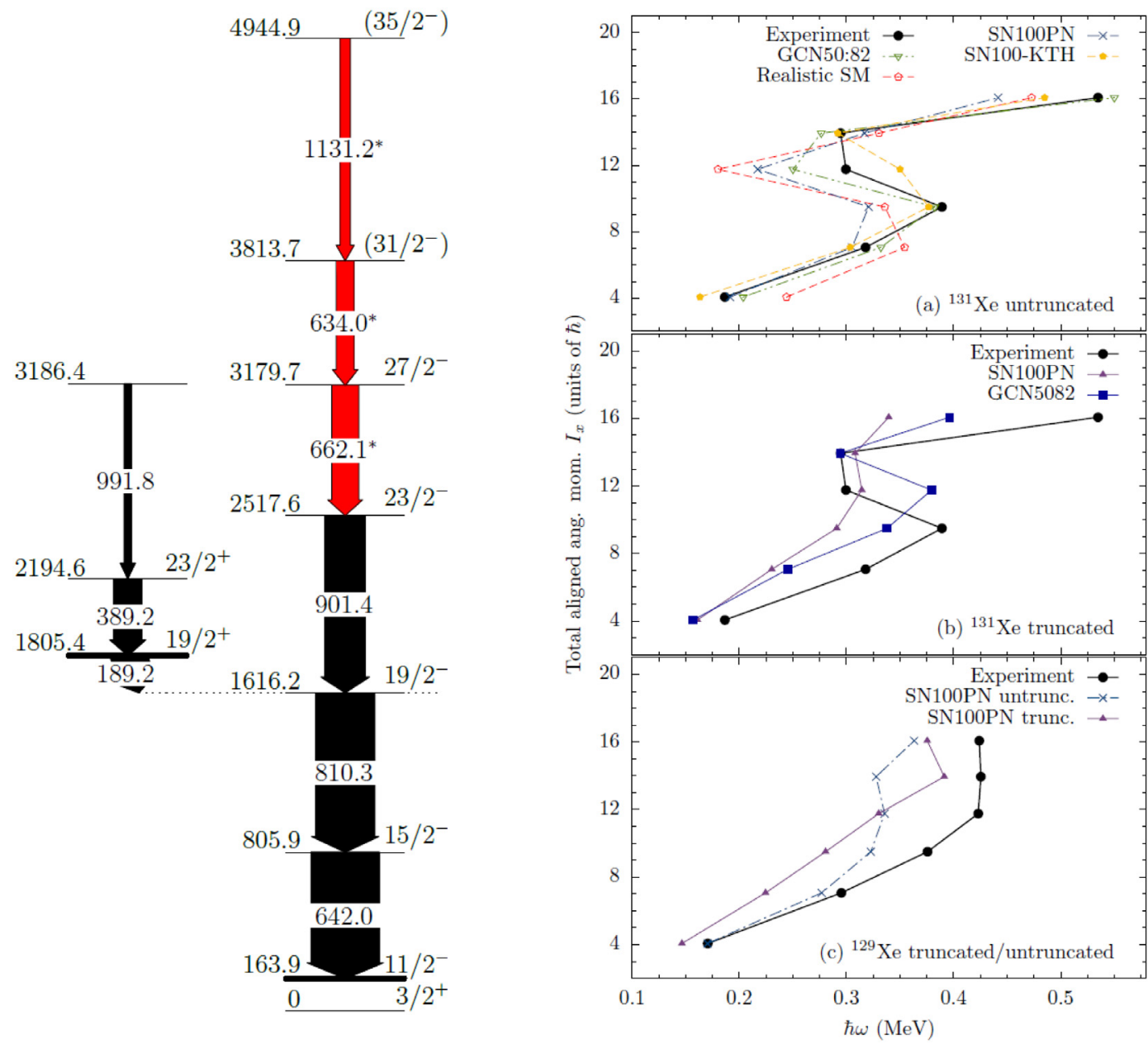

Fig. 60. Left panel: Extended level schemes of ${ }^{131}$ Xe. Right panel: (a) Comparison between experimental and calculated total aligned angular momenta $I_{x}$ as a function of the rotational frequency $\hbar \omega$, employing the SN100PN, GCN50:82, SN100-KTH, and realistic SM calculations for ${ }^{131}$ Xe. (b) Comparison employing the SN100PN and GCN50:82 with a truncation of only one allowed proton in the $\pi h_{11 / 2}$ orbital. (c) Similar comparison for ${ }^{129}$ Xe employing the SN100PN calculation: (i) untruncated and (ii) truncated with only one proton allowed in the $\pi h_{11 / 2}$ orbital.

Source: Experimental data for ${ }^{129} \mathrm{Xe}$ are taken from Ref [290]. Figures taken from Refs. [258].

by delayed-prompt coincidences. Shell-model calculations were performed as part of [255] reproduce the experimental findings remarkably well and corroborate most of the suggested assignments.

The AGATA MNT experiment [260] extended the level scheme of ${ }^{134} \mathrm{Ba}$. A distinct backbending along the positive-parity yrast band was identified at $\hbar \omega \approx 0.38 \mathrm{MeV}$. A systematic investigation of crossing frequencies along the even-Ba chain anticipate a neutron alignment at the position of the $J^{\pi}=10^{+}$state in ${ }^{134} \mathrm{Ba}$. Shell-model calculations with six different interactions were performed to elucidate the alignment structure of ${ }^{134} \mathrm{Ba}$. In general, backbending at the position of the $J^{\pi}=10^{+}$state is fairly reproduced for ${ }^{134} \mathrm{Ba}$. Moreover, a second alignment at the $J^{\pi}=16^{+}$state is predicted correctly by all calculations. The trend of calculated $B(E 2)$ values for transitions in the ground-state band is in agreement with the manifested neutron alignment in ${ }^{134} \mathrm{Ba}$.

The nucleus ${ }^{131}$ Xe lies in a transitional region between spherical $S n$ and well-deformed Ce nuclei. Along the Xe chain a rotational character is observed in the lighter $(A \leq 130)$ and shell-model character in the heavier $(A \geq 132)$ isotopes. A new upbend along the (negative signature $\alpha=-1 / 2$ ) negative-parity yrast band at the position of the $J^{\pi}=27 / 2^{-}$state was observed very recently in ${ }^{129} \mathrm{Xe}$ [290]. As a main result of the AGATA publication [258], the level scheme of ${ }^{131} \mathrm{Xe}$ was extended up to an excitation energy of $5 \mathrm{MeV}$ (see Fig. 60). A pronounced backbending along the negative-parity band on top of the one-quasiparticle $v h_{11 / 2}(\alpha=-1 / 2)$ band around $\hbar \omega=0.4 \mathrm{MeV}$ was identified. Angular-correlation measurements were performed to determine the multipole character of the $\gamma$-ray transitions.

In the last decades, the theoretical investigations of alignment properties were performed by means of collective approaches like interacting boson model [287,306,307], mean-field methods [286,308], or the cranked-shell-model [290, 309]. As a ramification of remarkable theoretical advances, the experimental findings in ${ }^{131} \mathrm{Xe}$ [258] were confronted 
with results of modern large-scale shell-model calculations considering the PQM130 by [209,305], SN100PN by [281], GCN50:82 by [301,302], Realistic SM by [303,304], and SN100-KTH by [310,311] interactions.

It was demonstrated that the new results, including the pronounced alignments in ${ }^{129,131} \mathrm{Xe}$, are well reproduced by the different shell-model interactions. Moreover, truncated calculations in ${ }^{129-132} \mathrm{Xe}$, prohibiting more than one proton in the $\pi h_{11 / 2}$ orbital, were compared with each other. The comparison between the different calculations revealed that the alignments in ${ }^{129,131}$ Xe can be microscopically traced back to a pair of $h_{11 / 2}$ protons, closing a gap in the alignment systematics along the Xe chain. Particularly, calculated $B(E 2)$ transition strengths in the negative-parity band are reduced for transitions between states were the alignment sets in. The outcomes of the work indicate that only interactions with improved and corrected monopole parts, i.e. GCN50:82, can describe the experiment alignment and crossing frequency to its full extent.

\subsection{Shapes of heavy neutron-rich nuclei}

For the neutron-rich osmium isotopes [312] one of the rare nuclear shape transitions from prolate to oblate deformation is predicted. They are part of the neutron-rich Pt-Os-W isotopes and they exhibit oblate deformation or non-axial symmetry (such as triaxiality) in the vicinity of the $\mathrm{N}=126$ closed shell. Therefore this region is of particular relevance for the question why prolate shapes predominate all over the chart of nuclei and for understanding the subtle interplay between single-particle and collective degrees-of-freedom. This is intimately related to the underlying NN interaction and its role in the microscopic origin of deformation.

Experimentally, the yrast band of ${ }^{194}$ Os was interpreted to be due to a prolate deformed nucleus [313], while the lowlying excitations of ${ }^{198}$ Os were suggested to be in line with an oblate shape [314]. First attempts to study the even-even nucleus in between, ${ }^{196}$ Os via fusion-evaporation reactions failed with stable beam and target combinations. Later ${ }^{196}$ Os was populated via fragmentation reactions and multi-nucleon transfer reactions. ${ }^{196}$ Os was produced via fragmentation reactions at GSI, Germany. However, no delayed $\gamma$-rays were observed in isomeric decay-spectroscopy experiments. In order to study the structure of ${ }^{196}$ Os [312] by means of $\gamma$-ray spectroscopy, a multi-nucleon transfer experiment was performed at LNL using AGATA in coincidence with the magnetic spectrometer PRISMA and a ${ }^{82}$ Se beam with an energy of $426 \mathrm{MeV}$ on a ${ }^{198} \mathrm{Pt}$ target. The very selective binary partner method was applied, i.e. the lighter beamlike recoil was identified in the magnetic spectrometer and the $\gamma$ rays of the corresponding heavier target-like recoil were detected in coincidence with it. In the $\gamma$-ray spectrum in coincidence with ${ }^{196} \mathrm{Os}$, three $\gamma$ rays were assigned to originate from the decay of the three lowest-lying $2^{+}, 4^{+}$, and $6^{+}$states. The $E\left(4_{1}^{+}\right) / E\left(2_{1}^{+}\right)$ratio was extracted to be 2.44 , very close to the value of a $\gamma$-soft triaxial rotor.

The excited states of ${ }^{196}$ Os as well as known levels in the even-even osmium isotopes ${ }^{188-198}$ Os were compared to new beyond mean-field calculations. The calculations were based on energy-density functionals that have been successfully applied to reproduce the collective character of the ground-state bands in the osmium and platinum isotopic chain. The symmetry-conserving configuration-mixing (SCCM) method includes simultaneous particle number and angularmomentum projections and axial and non-axial shape mixings. It yields information on both intrinsic deformations and the properties of excited levels. The quality of the results allowed a detailed analysis of the shape evolution from a prolate rotational in ${ }^{188} \mathrm{Os}$ to a vibrational one in ${ }^{198} \mathrm{Os}$. The new experimental data obtained for ${ }^{196} \mathrm{Os}$ revealed an almost perfect $\gamma$-soft triaxial rotor yrast-band, which is in very good agreement with the calculations.

A variety of other nuclei were produced in the ${ }^{82} \mathrm{Se}+{ }^{198} \mathrm{Pt}$ MNT reaction. The analysis of the $2 \mathrm{n}$ pick-up channel allowed to extend the level scheme of ${ }^{200} \mathrm{Pt}$ to higher spin values. The shape evolution of the even-even platinum isotopes ${ }^{190-200} \mathrm{Pt}$ [240] is compared to calculations based on the same nuclear model like for the osmium results (see Fig. 61).

The shape evolution through the platinum isotopes is less rapid than the one of the osmium isotopes, with a potential which remains $\gamma$-soft for all these nuclei. In particular, the yrast band of ${ }^{200} \mathrm{Pt}$ shows the most vibrational character among the investigated isotopes. The nuclear shape evolution of the even-even ${ }^{190-204} \mathrm{Pt}$ isotopes was studied via SCCM calculations. The theoretical predictions agree with the experimental data. In particular the ground-state band of ${ }^{200} \mathrm{Pt}$ is well reproduced, revealing its nature as a transitional nucleus between the lighter $\gamma$-unstable platinum isotopes and the presumably spherical $N=126$ platinum isotope ${ }^{204} \mathrm{Pt}$.

Rotational and vibrational modes of collective excitations of the neutron-rich actinide nucleus ${ }^{240} \mathrm{U}$ were investigated following multinucleon-transfer reactions [315]. $\gamma$-ray spectroscopy of ${ }^{240} \mathrm{U}$ was performed in kinematic coincidence, i.e. the binary $2 \mathrm{n}$ transfer channel ${ }^{134} \mathrm{Xe}$ was selected with PRISMA. The Doppler correction was then calculated assuming binary-partner kinematics. Time differences between PRISMA and DANTE were exploited to cope with the background and to suppress the overwhelming fission events. The ground-state band of ${ }^{240} \mathrm{U}$ was extended up to the $24^{+}$state (see Fig. 62).

Kinetic and dynamic moments of inertia were compared to theoretical predictions. An upbend at rotational frequencies of $\hbar \omega=0.2 \mathrm{MeV}$ was observed for the first time in the ground-state band. Various theoretical predictions were confronted with the experimental kinetic MoI (red data points versus black lines in left part of Fig. 63). For the model by Delaroche et al. [215] the absolute numbers of the kinetic MoI are consistently higher than the experimentally determined MoI. The macroscopic-microscopic model by Nerlo-Pomorska [213] underestimates the beginning of the experimental upbend. The cluster model by Shneidman et al. [214] does not reproduce the behavior at higher rotational frequencies. The cranked relativistic Hartree-Bogoliubov (CRHB) approach by Afanasjev and Abdurazakov [316,317] reproduces best the 

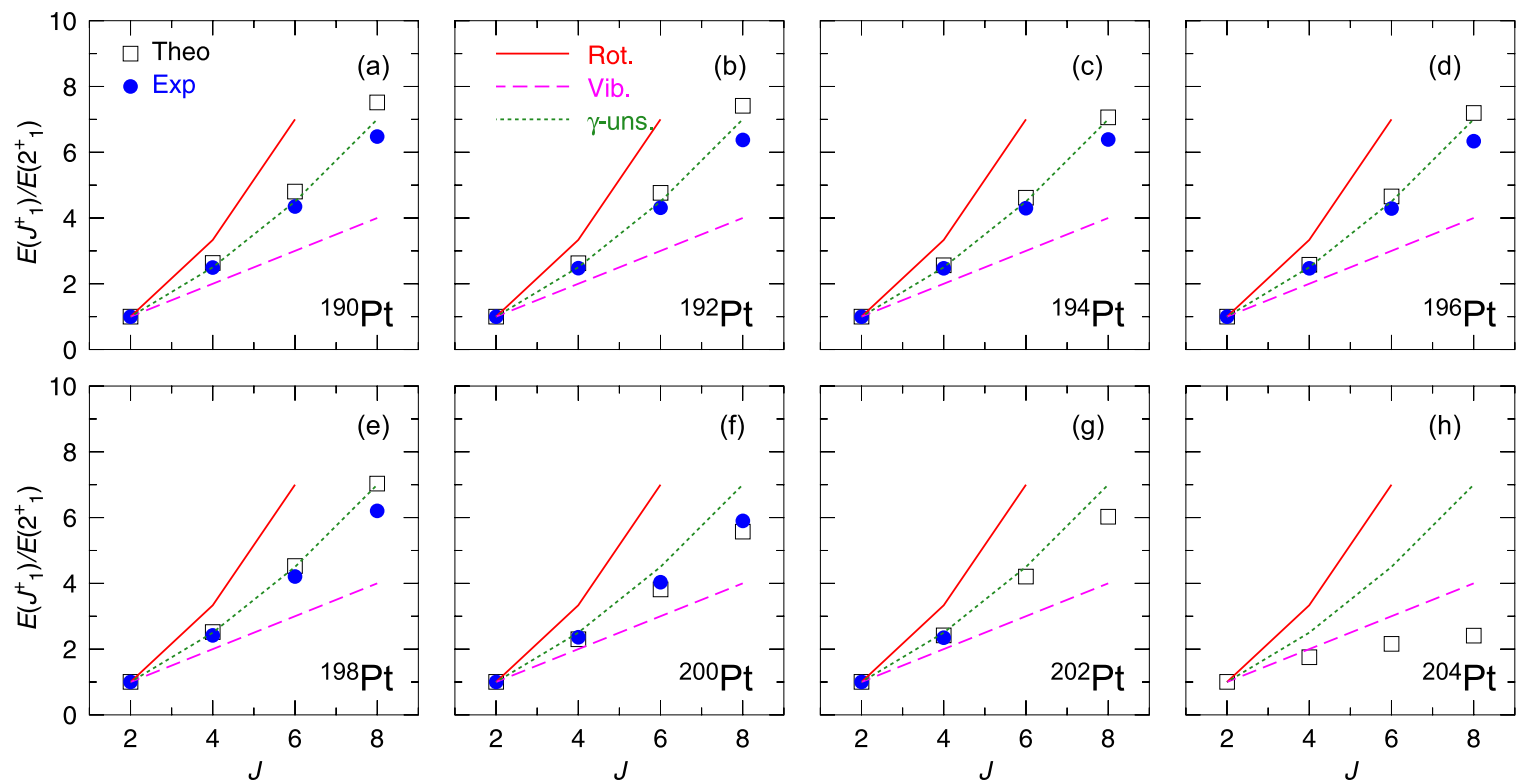

Fig. 61. Excitation energy ratio $\mathrm{E}\left(\mathrm{J}^{+}\right) / \mathrm{E}\left(2^{+}\right)$for the yrast $\mathrm{J}^{+}$and $2^{+}$states for the ${ }^{190-204}$ Pt even-even isotopes. The blue dots and the black squares correspond to the experimental points and SCCM predictions, respectively. Theoretical limits for the axial rotor (solid lines), the vibrator (dashed lines) and a $\gamma$-unstable or triaxial rotor (dotted lines) geometrical model are also plotted. The experimental data are taken from literature and from the present work for ${ }^{196-200} \mathrm{Pt}$ isotopes.

Source: Adapted from [240].
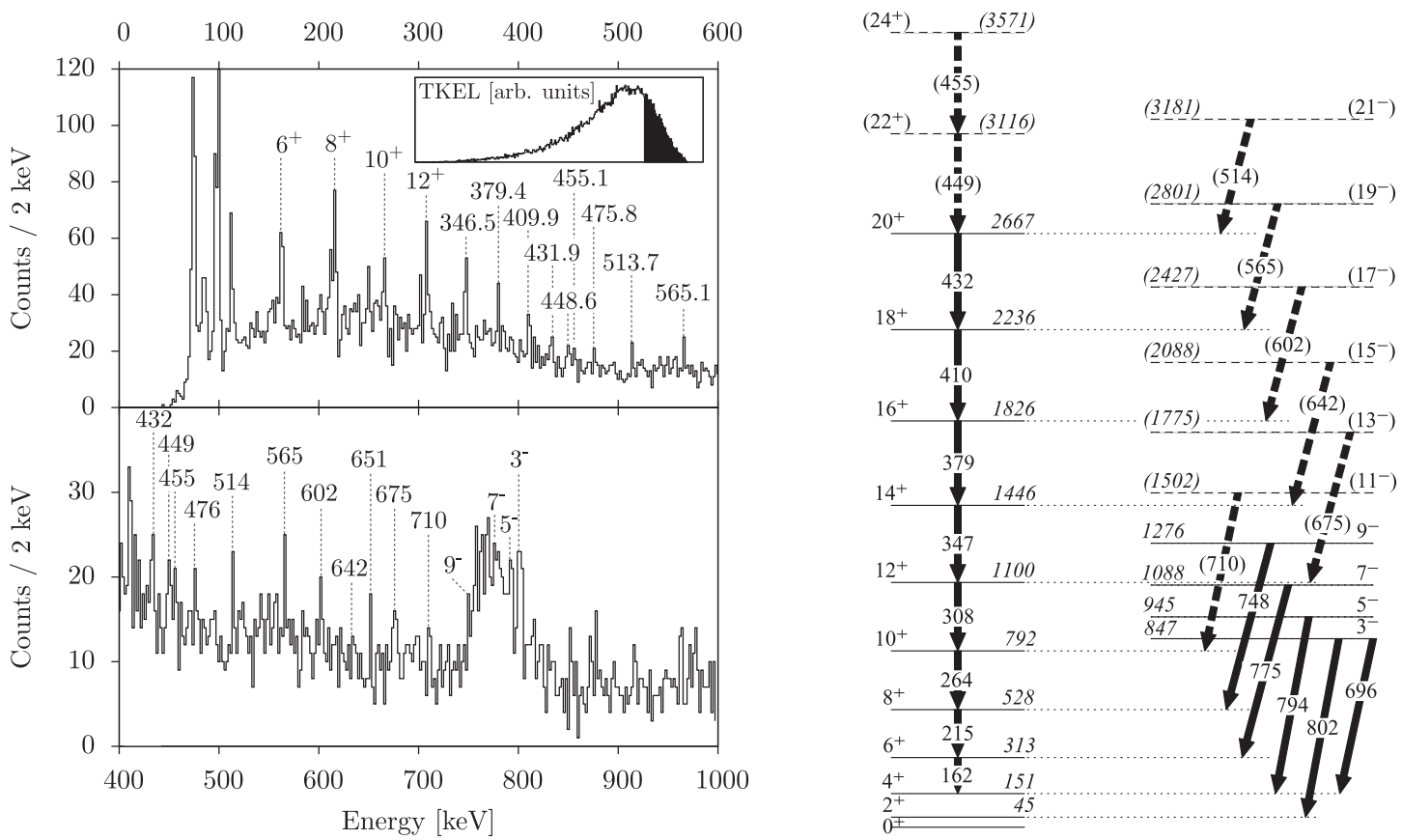

Fig. 62. Left panel: Doppler corrected single $\gamma$-ray spectrum for ${ }^{240} \mathrm{U}$ gated by ${ }^{134} \mathrm{Xe}$ identified in PRISMA. Besides the applied gate for MNT an additional cut on the TKEL value was performed (see black region in inset). Right panel: Proposed level scheme for ${ }^{240} \mathrm{U}$ that is based on $\gamma \gamma$-coincidence relationships. Tentative spin and parity assignments are given in brackets.

Source: Figures taken from Refs. [315].

experimental MoI. The $\mathrm{LN}\left(\mathrm{NL}^{*}\right)$ parametrization is in good agreement with the data points at lower spins, while at even higher spins the LN(NL1) parametrization provides the best agreement. Both calculations suggest a sharp increase of the 

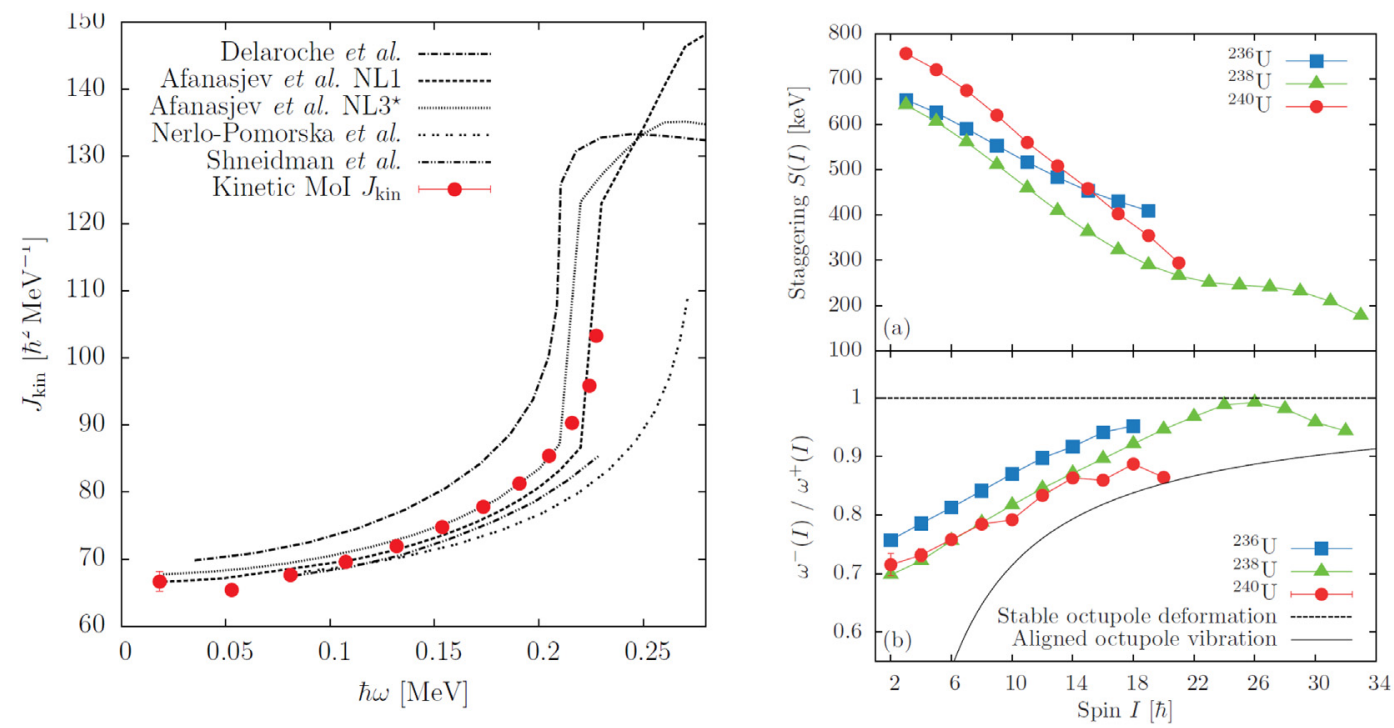

Fig. 63. Left panel: Kinetic MoI, $J_{\text {kin }}$, from this work (red points) in comparison to various theoretical predictions (black dotted lines). The CRHB + $\mathrm{LN}(\mathrm{NL} 1)$ and $\mathrm{CRHB}+\mathrm{LN}\left(\mathrm{NL}^{*}\right)$ calculations by Afanasjev et al. best reproduce the experimental data. Right panel: (a) Staggering S(I) in the three uranium isotopes ${ }^{236} \mathrm{U},{ }^{238} \mathrm{U}$ and ${ }^{240} \mathrm{U}$. The staggering parameter $\mathrm{S}(\mathrm{I})$ for ${ }^{240} \mathrm{U}$ continues to decrease up to the highest spins while $\mathrm{S}(\mathrm{I})$ saturates in the lighter $U$ isotopes (for details see text). (b) Ratio of rotational frequencies of the positive- and negative-parity bands as a function of spin. Source: Figures taken from Refs. [315].

kinetic Mol above $\omega \approx 0.2 \mathrm{MeV}$ due to the alignment of $i_{13 / 2}$ protons and $j_{15 / 2}$ neutrons which take place at similar rotational frequencies.

Despite the experimental challenges, the $K=0^{-}$negative-parity band was extended up to a tentatively assigned $21^{-}$ state. The parity-splitting, defined as the ratio of rotational frequencies of yrast and first negative-parity band, and the staggering of the states are clear indicators for the collective features and octupole correlations (see right part of Fig. 63). The staggering parameter $\mathrm{S}(\mathrm{I})$ displays to which extent the odd spin $I$ of the negative-parity band has an excitation energy located in between those of the two neighboring even-spin states with spins $I-1$ and $I+1$, therefore parametrizing to which extend the two bands of opposite parity can be regarded as a single, rotational octupole excitation (for more details see also [318]). At higher spins, ${ }^{240} \mathrm{U}$ is best interpreted as an aligned octupole vibrator, similar to the neighboring ${ }^{236} \mathrm{U}$ and ${ }^{238} \mathrm{U}$ isotopes.

\section{Probing symmetries in nuclei}

Symmetry properties showing up in nuclei, mainly with selection rules in their decay modes, have implications either on basic symmetries of the nucleon-nucleon interaction or on features of the configurations and energies for nuclear excitations based on the shell model, using effective mean-field forces and residual interactions.

Effects of the symmetry of the nucleon-nucleon interaction (denoted as isospin symmetry) manifest themselves in mirror nuclei (a pair of nuclei in which the values of $\mathrm{N}$ and $\mathrm{Z}$ are exchanged) and by the fact that for $\mathrm{N}=\mathrm{Z}$ nuclei the $\gamma$-decay of E1 type is forbidden. There is presently interest to validate these concepts either with an improved precision or in region of nuclear masses and excitations not yet explored.

Symmetry properties in nuclear configuration are, among others, those that give rise to degeneracy of single-particle states (denoted as pseudospin symmetry) and to excitation in phase of protons and neutrons (states denoted fully symmetric states). Furthermore, selection rules appear to be present in the decay of long-lived metastable states (isomeric states) in axially-deformed symmetric systems, away from closed shells, manifesting collective rotational properties. The description of these nuclei relies on the use of the $\mathrm{K}$ quantum number, $\mathrm{K}$ being the projection of the nuclear angular momentum on the symmetry axis of the nucleus. The conservation of this quantum number implies quenching of the E1 statistical transitions between low-K and high-K excited bands.

Several experiments were performed with AGATA to address symmetries properties and selection rules in nuclei and the effects due to their breaking, usually rather small and thus difficult to be detected. In the following the motivation and results of these experiments are reported.

\subsection{Isospin symmetry and isospin properties}

The concept of isospin was introduced by Werner Heisenberg in 1932 [319]. He used it to explain the similarities between the proton and the newly discovered neutron. The term itself was introduced by Eugene Wigner in 1936 [320]. 

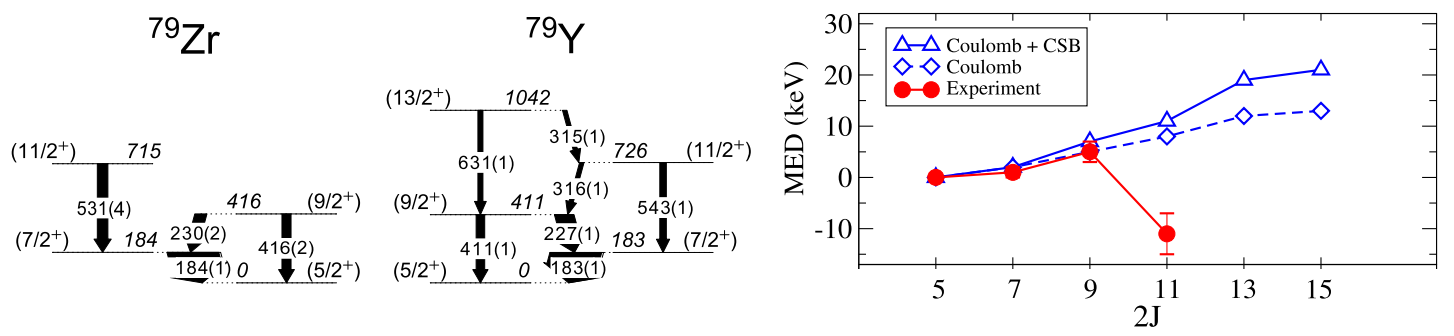

Fig. 64. Left panel: The level schemes of mirror nuclei ${ }^{79} \mathrm{Zr}_{39}$ and ${ }^{79} \mathrm{Y}_{40}$. Right panel: Mirror energy differences (MED) as function of spin. The experimental values are compared with theoretical calculations which include Coulomb only and Coulomb plus charge symmetry breaking terms. Source: Figure adapted from [322].

Protons and neutrons have very similar properties (except the charge) and can be grouped together as nucleons. Therefore, it is convenient to treat them as same particles. They have isospin $T=1 / 2$, and protons and neutrons differ by their projections, $T_{z}=-1 / 2$ and $1 / 2$, respectively (the choice of sign is just a convention). The strong nuclear force is charge independent. This charge symmetry requires that the nucleon-nucleon force is isospin dependent. States with the same isospin in a set of nuclei with the same number of nucleons $A$ are called isobaric analogue states. These can be easily studied around the $\mathrm{N}=\mathrm{Z}$ line. Their similarity, as manifested by the level schemes of mirror nuclei is a powerful proof of the charge independence of the nuclear force and the usefulness of the concept of the isospin [321]. Isospin non-conserving forces, such as the Coulomb force, are relatively weak.

The experiments performed with AGATA, described below, have addressed three relevant issues related to the isospin degree of freedom : (i) the proton transition matrix in isobaric analogue states and (ii) isospin mixing from the decay of giant dipole resonance in compound nuclei; (iii) proton-neutron pairing with isospin zero.

\subsubsection{Mirror nuclei and the proton transition matrix in isobaric analogue states}

A traditional test of isospin symmetry is based on comparing the level schemes of mirror nuclei. The heaviest mirror nuclei accessible are ${ }^{79} \mathrm{Y}_{40}$ and ${ }^{79} \mathrm{Zr}_{39}$, recently populated in nucleon knock-out reactions and studied using the GRETINA tracking array [322]. The two level schemes are extremely similar, as shown in Fig. 64. The difference in the energy levels (Mirror Energy Differences) are also presented as function of spin. Due to the large number of valence particles, the usefulness of the shell-model is limited in this mass region. A new density functional approach was used to explain the experimental data. The calculations considered isospin-symmetry breaking effects originating from both Coulomb and nuclear charge-symmetry breaking terms. An excellent agreement between theory and experiment was obtained, especially for the lowest members of the band.

Transition strength measurements provide information on the wave functions of the involved states, therefore, providing more stringent test to theories. In the limit of pure isospin (no isospin mixing) the proton transition matrix elements for a set of analogue $T \rightarrow T$ electromagnetic transitions should be linear with $T_{z}$. This was tested by determining with high precision the lifetime, and consequently the $B\left(E 2 ; 2^{+}, T=1 \rightarrow 0^{+}, T=1\right)$ transition strengths for a set of nuclei with $T_{z}=-1,0,+1$.

The lifetimes of the $2^{+}$excited states in the $\mathrm{A}=46$ isobaric multiplet members ${ }_{24}^{46} \mathrm{Cr}-{ }_{23}^{46} \mathrm{~V}-{ }_{22}^{46} \mathrm{Ti}$ were determined at GSI. The nuclei of interest were all produced from the fragmentation of an $E / A=600 \mathrm{MeV}{ }^{58} \mathrm{Ni}$ beam on a ${ }^{9} \mathrm{Be}$ target, and they impinged on a secondary gold target where they were Coulomb excited. The use of the same reaction mechanism for all three cases resulted in reduced systematic uncertainties. The lifetime was determined either via Coulomb excitation $\left({ }^{46} \mathrm{Cr}\right.$ and ${ }^{46} \mathrm{Ti}$, see Fig. 15) or from line-shape analysis following the Coulomb excitation on a stack of three targets $\left({ }^{46} \mathrm{Ti}\right.$ and ${ }^{46} \mathrm{~V}$, see Fig. 16). Since the less exotic ${ }^{46} \mathrm{Ti}$ was measured in both techniques, its well known B(E2) value could be used as reference point. The obtained values were combined with previously available data. The determined relative proton matrix elements, obtained from the B(E2) values, are shown in Fig. 65.

Shell-model calculations using the KB3G interaction [323] using the $f p$ model space $(20<Z<40$ and $20<N<40)$ are shown in blue on the same figure. However, the measured constant trend of the $M_{p}$ proton matrix element is not reproduced. This indicates that the model space is not large enough and lower-lying single-particle orbitals need to be considered. Indeed, the values calculated within a model space comprising the $s_{1 / 2}, d_{3 / 2}, f_{7 / 2}$ and $p_{3 / 2}$ orbitals $(14<Z<32$ and $14<N<32$ ) with the ZBM2 interaction [324] reproduces the experimental trend very well. Nevertheless even in this case the calculated absolute magnitude of the $\mathrm{B}(\mathrm{E} 2)$ values are too low. The quadrupole strength is mainly due to coupling of $\Delta l=2$ orbitals, therefore the inclusion of the higher-lying $f_{5 / 2}, p_{1 / 2}$ and lower-lying $d_{5 / 2}$ orbitals would increase the theoretical values. This measurement, devoted to the heaviest $T=1$ triplet which is reachable for lifetime studies, provided the high precision test to the rule that, in the limit of pure isospin, the electromagnetic matrix elements show a linear behavior with $T_{z}$. 


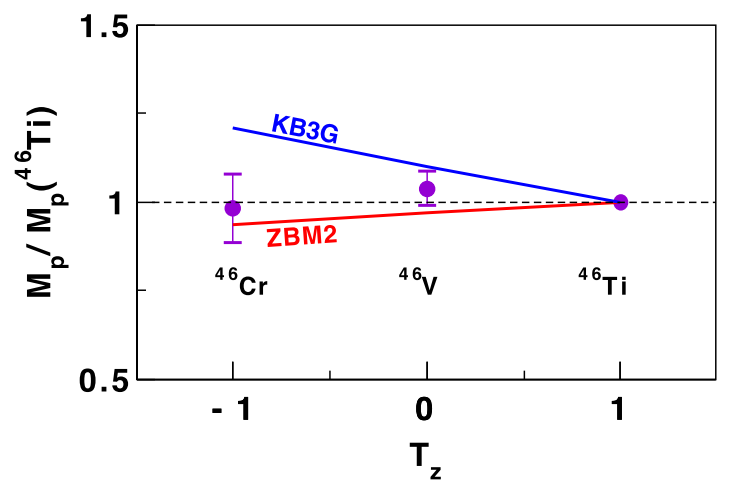

Fig. 65. The measured proton matrix element for the $\mathrm{A}=46{ }^{46} \mathrm{Cr}-{ }^{46} \mathrm{~V}-{ }^{46} \mathrm{Ti}$ isobar multiplet, plotted relative to that of ${ }^{46} \mathrm{Ti}$. The solid lines correspond to two different shell-model calculations, as explained in the text. Source: Figure taken from [54].

\subsubsection{Isospin mixing at finite temperature for $Z=40$}

It is well known that the breaking of isospin symmetry can be observed by decays which are forbidden by the isospin selection rules. This is the case for the E1 decay from excited states in self-conjugate nuclei. This property of the E1 decay can be fully exploited by going in the $\gamma$-ray region of the Giant Dipole Resonance where the maximum strength of the E1 transitions is concentrated. This approach can be employed for the E1 decay of the GDR in nuclei at finite temperature T, formed in fusion-evaporation reactions.

The GDR in self conjugate nuclei at finite temperature was up to now investigated in few experimental and theoretical works (see e.g. [325-328]). Self-conjugate compound nuclei at high excitation energy can be formed using fusionevaporation reactions using projectiles and targets with $\mathrm{N}=Z$. This condition ensures population of $\mathrm{CN}$ with isospin $\mathrm{I}=0$ and thus with hindered E1 decay from the GDR. In contrast, if the initial state is not pure in isospin but contains admixtures of $\mathrm{I}=1$ states, it can decay to the more numerous final states with isospin $\mathrm{I}=0$. At finite temperature there is the additional effect of a partial restoration of the isospin symmetry. This is because the degree of mixing in a compound nucleus is limited by its finite lifetime for particle decay. Furthermore, it is particularly interesting to investigate isospin mixing in nuclei with $\mathrm{N}=\mathrm{Z}$ in the mass region $\mathrm{A}=70-100$ because there the different model predictions have the largest discrepancies among them (see e.g. [329]).

The experiment performed with the AGATA set up at LNL concerned the compound nucleus ${ }^{80} \mathrm{Zr}$ which was populated using the fusion reactions ${ }^{40} \mathrm{Ca}+{ }^{40} \mathrm{Ca}$ at a bombarding energy of $136 \mathrm{MeV}$. The $\gamma$-ray spectrum measured for this reaction is shown in the top left panel of Fig. 66 together with the statistical model predictions in which the only free parameter used to fit the data is the Coulomb spreading width. In the top right panel of Fig. 66 the reduced $\chi^{2}$ is shown, which has a well defined minimum. The other parameters entering in the statistical-model calculations were fixed by the analysis of the spectrum from the reaction ${ }^{37} \mathrm{Cl}+{ }^{44} \mathrm{Ca}$ at beam energy of $95 \mathrm{MeV}$, leading to a compound nucleus at the same temperature (see Section 5.3) and for which the isospin selection rule does not apply. An important step of the analysis was also the observation of the population of residual nuclei, that was compared to the statistical model under different gating conditions (see [196] and [195]).

The value of the isospin mixing parameter $\alpha$, related to the Coulomb spreading width, was obtained from the statisticalmodel analysis of the $\gamma$-ray spectrum measured with the ${ }^{40} \mathrm{Ca}+{ }^{40} \mathrm{Ca}$ reaction. It is shown with the black-diamond in the bottom panel of Fig. 66. The datum from [327] is also shown in the figure with the red circle, while the blue triangle is the theoretical prediction from [329]. The curves are calculations of isospin mixing at finite temperature for the ${ }^{80} \mathrm{Zr}$ nucleus based on the model reported in [328]. This model, which was found to describe the experimental findings in a reasonable way, made it possible to infer from the measurement at finite temperature the isospin mixing at zero temperature. To be noted that ${ }^{80} \mathrm{Zr}$ is an unstable proton-rich nucleus not easily accessible to find $\mathrm{E} 1$ transitions for it.

It is very interesting to connect the isospin mixing parameter with the isospin-correction term $\delta_{c}$ needed for $\beta$-decay calculations, introduced in Ref. [330]. This reference gives a simple linear relation among them. The isospin mixing parameter measured via the GDR $\gamma$ decay from ${ }^{80} \mathrm{Zr}$ yields the value shown with the red star in the inset of the bottom panel of Fig. 66. The other points in the insets are from mass and $\beta$-decay measurements and the predictions are from [330]. This datum from the GDR decay addresses a mass region not yet investigated and relevant to extrapolate theory in the region of ${ }^{100} \mathrm{Sn}$. More data with radioactive beams should be obtained for larger $\mathrm{Z}$ when the next generation of beams with larger intensities will become available.

\subsection{3. $T=0$ neutron-proton pairing}

Pair correlations are important in many fields of physics (e.g. the Bardeen-Cooper-Schrieffer theory of superconductivity). In nuclei pairing correlations manifest themselves in effects like increased binding energies for nuclei with even 

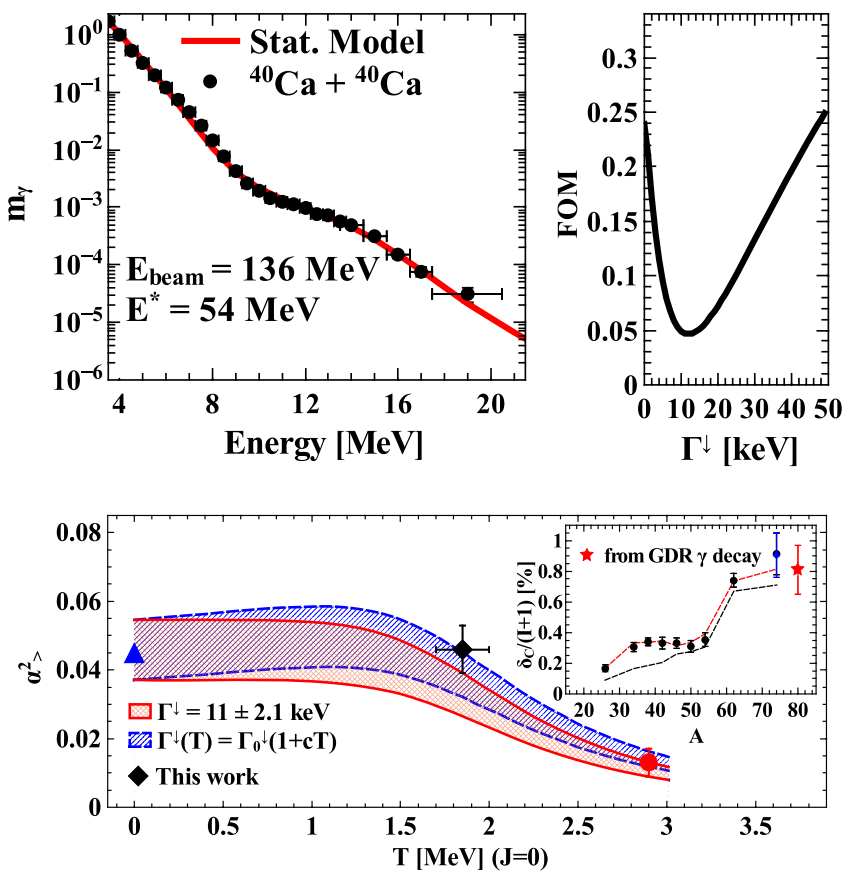

Fig. 66. Top panels: High energy $\gamma$-ray spectrum ( $\mathrm{m}_{\text {gamma }}$ on the vertical axis being the $\gamma$-ray multiplicity) for the reaction ${ }^{40} \mathrm{Ca}+{ }^{40} \mathrm{Ca}$ at a bombarding energy of $136 \mathrm{MeV}$. The data are shown with full circles in comparison with the best-fitting statistical-model calculation (red line). Top right panel: Figure of Merit (FOM) obtained by varying the Coulomb spreading width in the statistical-model calculations for the ${ }^{40} \mathrm{Ca}+{ }^{40} \mathrm{Ca}$ reaction. The FOM is the $\chi^{2}$ divided by the number of counts. Bottom panel: The isospin mixing $\alpha_{>}^{2}$ as a function of T obtained with the model of Ref. [328] and using the Coulomb Spreading width $\Gamma^{\downarrow}$ given in the legend. The blue triangle is the theoretical value at $T=0$ from Ref. [329], the red circle is the datum from Ref. [327], and the black diamond is the datum from AGATA. The inset gives the isospin mixing correction as a function of the nuclear mass A. The black line is the prediction from the Damgaard model [331], while the red line is a shell-model prediction with a Saxon-Woods radial wave function [332]. Black circles are data extracted from $\beta$ decay [333], the blue diamond is from the mass measurement [334], and the red star is from the AGATA work.

Source: Adapted from Ref. [196].

number of protons and even number of neutrons. As seen in the previous section, the strong nuclear force is charge independent. But it is also spin dependent, resulting in isospin dependence. This means that proton-proton, neutronneutron and neutron-proton pairs with isospin $\mathrm{T}=1$ behave in the same way. However proton-neutron pairs can couple to $\mathrm{T}=0$. The existence of the deuteron with spin $\mathrm{I}=1$, and the unbound nature of both two-proton and two-neutron systems demonstrates that the $\mathrm{T}=0, \mathrm{I}=1$ correlation is stronger than the $\mathrm{T}=1, \mathrm{I}=0$ ones. In heavier nuclei with equal number of protons and neutrons, occupying the same single-particle orbitals, enhanced neutron-proton correlations might be present. These are called isoscalar $(T=0)$ neutron-proton pair. However, there is no conclusive evidence for the need to include such correlated $\mathrm{T}=0$ proton-neutron pairs in the calculations in order to describe the properties of such nuclei [335].

Medium to high-spin states in rotating $\mathrm{N}=\mathrm{Z}$ nuclei are considered to be among the best places to search for $\mathrm{T}=0$ neutron-proton pairing. The isoscalar neutron-proton coupling is less affected by the Coriolis interaction, which would result in a delayed band crossing when compared to the neighboring nuclei. Heavier nuclei are better candidates, but experimentally more difficult to study them.

The $\mathrm{N}=\mathrm{Z}{ }^{88} \mathrm{Ru}$ nucleus was studied recently in an AGATA experiment at GANIL [336]. It was populated in the ${ }^{54} \mathrm{Fe}\left({ }^{36} \mathrm{Ar}, 2 \mathrm{n} \gamma\right)$ fusion-evaporation reaction. The experiment lasted 13 days (longer than typical) with an average beam intensity of $7 \mathrm{pnA}$. The population cross section of ${ }^{88} \mathrm{Ru}$ is very low, therefore ancillary detectors to detect evaporated neutrons and charged particles were used. The neutrons were detected with the NEDA [67] and Neutron Wall arrays [68], while charged particles were detected with the DIAMANT array [69] consisting of CsI(Tl) detectors. NEDA and Neutron Wall allow neutron/gamma discrimination by the use of pulse-shape discrimination and time-of-flight measurement. DIAMANT, covering almost the full $4 \pi$ solid angle can distinguish between different charged particles.

The $\gamma$-ray spectrum for ${ }^{88} \mathrm{Ru}$ is shown in Fig. 67. It was obtained by selecting known ${ }^{88} \mathrm{Ru} \gamma$ rays [337] in the $\gamma \gamma$ matrix, with additional coincidence conditions requiring two-neutrons and no charged particles in the same event. The band crossing frequency can be determined from Fig. 68 showing the kinematical moment of inertia as a function of the rotational frequency. Both of these quantities are determined from the $\gamma$-ray energies and the spins of the states from which they originate. Consequently, the correct ordering of the transitions is crucial; it is based on the measured intensities, which decrease at higher spins. The extended level scheme, with the three new transitions allowed the 

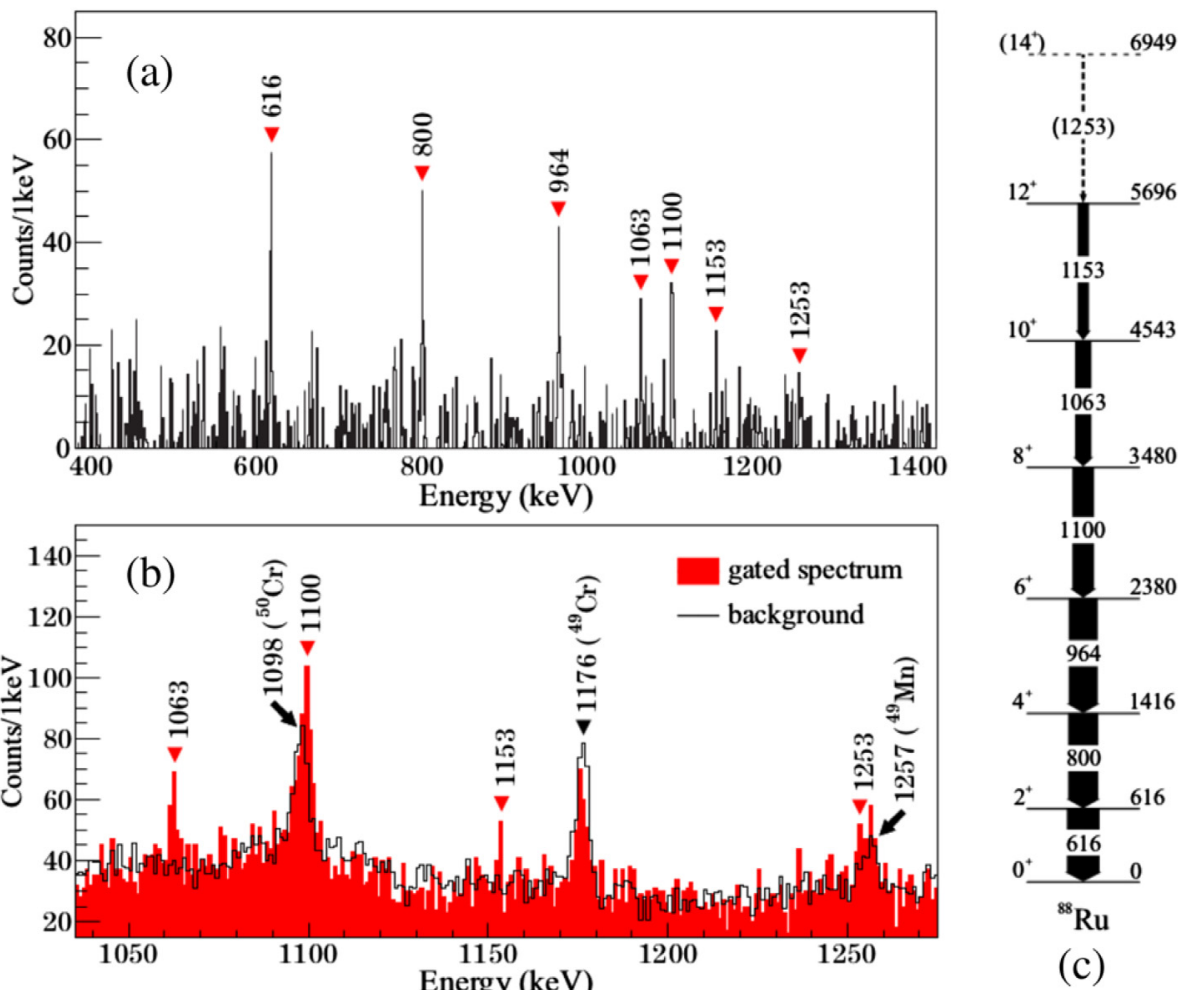

Fig. 67. Top left panel: (a) $\gamma$-ray energy spectrum of ${ }^{88} \mathrm{Ru}$. It was obtained in coincidence with any of the known $616,800,964$, and $1100 \mathrm{keV} \gamma$ rays of ${ }^{88} \mathrm{Ru}$ [337] and two neutrons, and in anti-coincidence with charged particles. Bottom left panel: (b) The same energy spectrum indicating the new transitions at 1063, 1153 and $1253 \mathrm{keV}$ is shown in red together with the background spectrum in black. Contaminants, arising from reactions on oxygen, are indicated. Right panel: (c) Level scheme of ${ }^{88} \mathrm{Ru}$.

Source: Figure taken from [336].

determination of the band crossing frequency at $\hbar \omega \sim 0.55 \mathrm{MeV}$. This is significantly larger than those observed in the lighter $\mathrm{N}=44$ isotones ${ }^{84} \mathrm{Zr}$ and ${ }^{86} \mathrm{Mo}$. In these nuclei the band crossing is explained by the alignment of two $g_{9 / 2}$ protons, and cranked shell-model calculations reproduce the rotational frequency where it occurs. Large-scale shell-model calculations including $T=0$ neutron-proton pairing predict different behavior in these systems, with a delayed alignment in the $\mathrm{N}=\mathrm{Z}{ }^{88} \mathrm{Ru}$ nucleus compared to ${ }^{86} \mathrm{Mo}$ and ${ }^{84} \mathrm{Zr}$, in line with the experimental results. Consequently, the reported results were interpreted as evidence of the presence of isoscalar neutron-proton pairing in ${ }^{88} \mathrm{Ru}$.

\subsection{Symmetries and selection rules for nuclear configurations}

\subsubsection{Pseudospin symmetry}

The concept of pseudospin symmetry is based on near degeneracy of single-particle states with quantum numbers $n, l, j=l+1 / 2$ and $n-1, l+2, j=l+3 / 2$ (note that they have the same parity). Therefore pseudospin doublets are defined by quantum numbers: $\tilde{n}=n-1, \tilde{l}=1+1, \tilde{j}=1 \pm 1 / 2$ [338]. Pseudo-SU(3) symmetry can be successfully applied for nuclei north-east of ${ }^{78} \mathrm{Ni}$. The neutron orbitals above $\mathrm{N}=50$ can be grouped into $\tilde{f}$ (formed by $2 d_{5 / 2}$ and $\left.1 g_{7 / 2}\right)$ and $\tilde{p}\left(3 s_{1 / 2}\right.$ and $\left.2 d_{3 / 2}\right)$ pseudospin doublets, while the protons above $Z=28$ form $\tilde{s}\left(p_{1 / 2}\right.$ without a partner) and a $\tilde{d}\left(2 p_{3 / 2}\right.$ and $\left.1 f_{5 / 2}\right)$ doublet.

In an experiment performed at GANIL the $\mathrm{N}=52$ isotones ${ }^{88} \mathrm{Kr},{ }^{86} \mathrm{Se}$ and ${ }^{84} \mathrm{Ge}$ were populated from a ${ }^{238} \mathrm{U}$ beam at $\mathrm{E} / \mathrm{A}=6.2 \mathrm{MeV}$ impinging on a ${ }^{9} \mathrm{Be}$ target $[339,340]$. The fission products passed through a $\mathrm{Mg}$ foil then entered into the VAMOS++ spectrometer [30] where they were identified. The prompt $\gamma$ rays were detected by AGATA placed at backward angles. The Doppler shift of a given $\gamma$ ray depends on the velocity of the emitting nucleus. The Doppler shift is larger if the photon is emitted before the nucleus reaches the Mg foil, and lower after passing the foil. The ratio of the intensities of the two components depends on the lifetime of the excited state. Measurements were made for three target-degrader distances: 120, 270, and $520 \mu \mathrm{m}$, and the lifetimes were obtained by using the so-called recoil distance Doppler shift method [63].

Several lifetimes, including those of the $2^{+}$and $4^{+}$yrast states, were determined in the $\mathrm{N}=52{ }^{88} \mathrm{Kr},{ }^{86} \mathrm{Se}$ and ${ }^{84} \mathrm{Ge}$ isotones $[339,340]$. As illustration, the mean-lives obtained for the first time for the most exotic system, ${ }^{84} \mathrm{Ge}$, were 


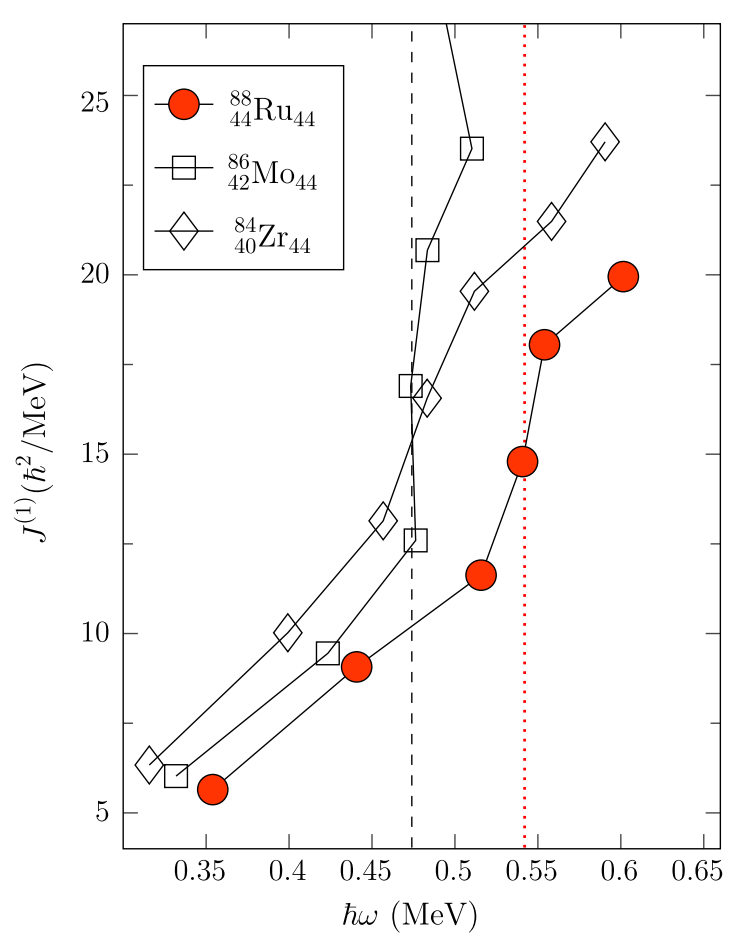

Fig. 68. Kinematical moment of inertia as function of rotational frequency for the low-lying yrast bands of the $\mathrm{N}=44$ isotones ${ }^{88} \mathrm{Ru}$, ${ }^{86} \mathrm{Mo}$ and ${ }^{84} \mathrm{Zr}$. The red dotted vertical line indicates the band crossing frequency for ${ }^{88} \mathrm{Ru}$. The black dashed vertical line indicates the approximate rotational frequency of the first isovector-paired band crossing due to $g_{9 / 2}$ protons as predicted by standard cranked shell-model calculations.

Source: Figure taken from [336].

$\tau\left(2^{+}\right)=1.3_{-6.5}^{+3.0} \mathrm{ps}$ (effective) and $\tau\left(4^{+}\right)=232.9_{-52.5}^{+398.4} \mathrm{ps}$. The $B\left(E 2 ; 2^{+} \rightarrow 0^{+}\right)$reduced transition strengths for the $\mathrm{N}=52$ even-even nuclei are shown in Fig. 69. Naively one would expect that the $\mathrm{B}(\mathrm{E} 2)$ values, a measure of the collectivity, decrease as one approaches the $\mathrm{Z}=28$ closed shell; in other words one would expect lower collectivity in ${ }_{32} \mathrm{Ge}$ than in ${ }_{34} \mathrm{Se}$. In stark contrast, the new experimental value suggests increased collectivity in $\mathrm{Z}=32 \mathrm{Ge}$ compared to $\mathrm{Z}=34 \mathrm{Se}$. The experimental uncertainties for ${ }^{84} \mathrm{Ge}$ are large, nevertheless there is $\approx 85 \%$ probability that this is the case.

The $\mathrm{B}(\mathrm{E} 2)$ values measured in ${ }^{86} \mathrm{Se}$ and ${ }^{88} \mathrm{Kr}$ are well reproduced by shell-model calculations assuming an inert ${ }_{28}^{78} \mathrm{Ni}_{50}$ core. The same is true for the pseudo-SU(3) symmetry calculations [341]. This means that collectivity in these nuclei can be accounted for by considering the $\mathrm{Z}=20-28$ and $\mathrm{N}=50-70$ valence space. In contrast both calculations underpredict the value obtained for ${ }^{84} \mathrm{Ge}$. This would mean that fraction of collectivity arises from within the ${ }^{78} \mathrm{Ni}$ core, most likely the $v g_{9 / 2}$ neutrons can be excited across the $\mathrm{N}=50$ shell. By considering the excitation of a pair of such neutrons into the neutron $\tilde{p} \tilde{f}$ group, the measured ${ }^{84} \mathrm{Ge}$ value is reproduced (denoted $g_{9 / 2} \mathrm{P}-\mathrm{SU}(3)$ in Fig. 69 ). The implication is that there is a shape transition between the prolate deformed $\mathrm{Z}=32$ and the soft triaxial $\mathrm{Z}=34$ nucleus at $\mathrm{N}=52$. The only theory which reproduced the trend of collectivity in the $\mathrm{N}=52$ isotones is the mean-field calculation based on the relativistic functional DD1-PC1 [342]. In this case the increased quadrupole deformation is triggered by the proton orbital arrangements. However even in this case the slope is much lower than the experimental one. By looking at the evolution of the pseudo-spin orbits as function of isospin it was concluded that the single-particle arrangements triggering quadrupole coherence in ${ }^{84} \mathrm{Ge}$ originates from pseudo-spin symmetry. The suggestion fits into the wider picture that shape coexistence and shape evolution are important phenomena around, and indeed in, ${ }^{78} \mathrm{Ni}[129]$.

\subsubsection{Mixed-symmetry states}

Mixed-symmetry states are a general feature of two-component systems. They are usually defined in the framework of IBM-2, the version of the Interacting Boson Model which distinguishes between proton and neutron bosons. Protonneutron mixed symmetry one-quadrupole phonon excitations $\left(2_{m s}^{+}\right.$states) are expected in vibrational nuclei, spherical nuclei with few proton and neutron pairs outside the closed shell. They can be understood as out of phase excitations of protons and neutrons. In contrast the fully-symmetric yrast $2^{+}$is the result of in-phase excitation of protons and neutrons. The main signature of the $2_{m s}^{+}$state is the strong M1 transition to the yrast $2^{+}$state, with a matrix element of $\left\langle 2_{m s}^{+}|M 1| 2^{+}\right\rangle \approx 1 \mu_{N}[343]$. The first candidates of such states were identified in $\mathrm{N}=84$ isotones.

Theoretical calculations performed in the framework of IBM-2 suggested that $\alpha$-transfer reactions would populate $2_{m s}^{+}$states with large cross sections [344]; a population with an intensity of $1 / 3$ as compared to that of the yrast $2^{+}$was 


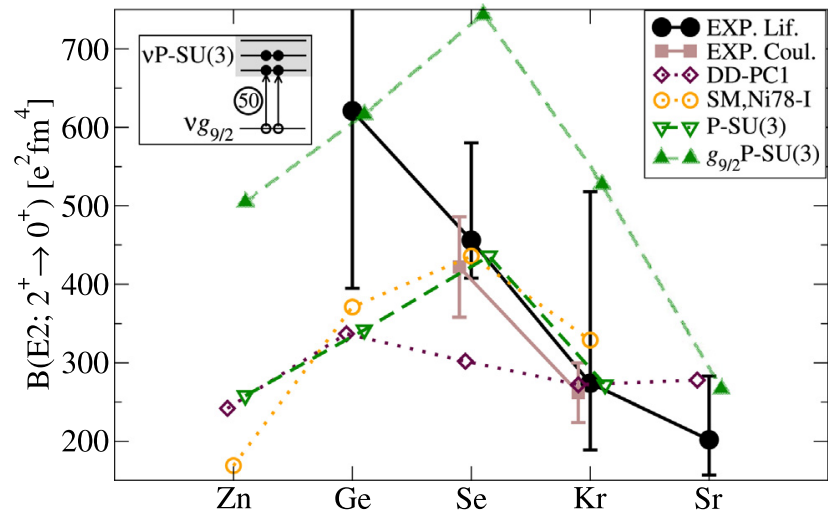

Fig. 69. $B\left(E 2 ; 2^{+} \rightarrow 0^{+}\right)$transition strengths for light even-even $\mathrm{N}=52$ isotones. Experimental and theoretical values are shown. For details see the text.

Source: Figure taken from [339].

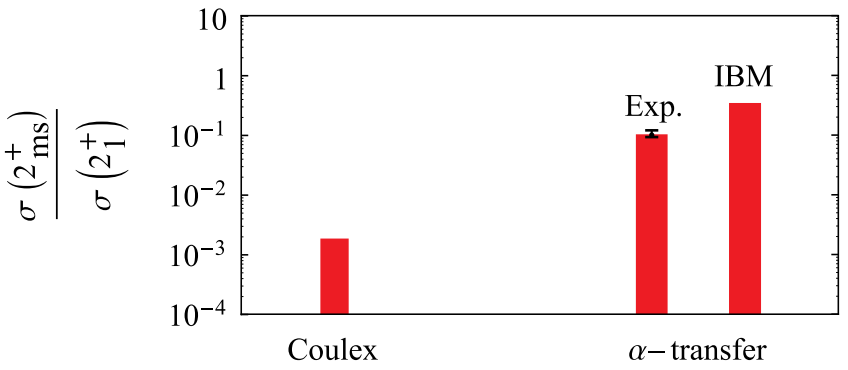

Fig. 70. Ratio of the cross sections for population of the $2_{m s(3)}^{+}$and $2_{1}^{+}$states in ${ }^{140} \mathrm{Ba}$. The ratio measured in $\alpha$-transfer reaction is compared with theoretical predictions and those from Coulomb excitation.

Source: Figure taken from [345].

predicted. This value is rather large for a state far from the yrast line. In order to test this hypothesis an AGATA experiment was performed at LNL [345]. A ${ }^{136} \mathrm{Xe}$ beam impinged on a natural carbon target. The ${ }^{12} \mathrm{C}\left({ }^{136} \mathrm{Xe},{ }^{140} \mathrm{Ba}\right){ }^{8} \mathrm{Be}$ reaction channel was selected by the detection of two coincident $\alpha$ particles in an annular double-sided silicon-strip detector. (Note that ${ }^{8} \mathrm{Be}$ is particle unstable, and therefore instantly disintegrates in two $\alpha$ particles.) In ${ }^{140} \mathrm{Ba}$ the $2_{3}^{+}$state at $1994 \mathrm{keV}$ is the candidate for the mixed-symmetry state. It is based on the small M1/E2 multipole mixing ratio of the $2_{3}^{+} \rightarrow 2_{1}^{+}$transition.

The $\gamma$-rays detected by AGATA provided information about the relative population of different states in ${ }^{140} \mathrm{Ba}$. The extracted population cross section of $2_{m s}^{+}$state relative to the $2_{1}^{+}$state is shown in Fig. 70 . The mixed-symmetry state was found to be populated $10.4(10) \%$ relative to the yrast $2^{+}$. This is much stronger when compared to the alternative Coulomb excitation reaction. However, it is only one third of the theoretical prediction. In addition, the second $2_{2}^{+}$state was populated with higher probability than the third $2_{3}^{+}$which is the mixed symmetry state. Consequently, it was established that $\alpha$-transfer reactions are a powerful tool to populate $2_{m s}^{+}$states. But the population of a $2^{+}$level in such a reaction does not provide unambiguous information on the character of the state.

\subsection{Isomeric states and selection rules}

Excited states in nuclei usually have very short lifetimes, in the order of picoseconds or even less. In contrast, isomeric (or metastable) states live much longer [346]. In addition of being special, the measurement of the lifetimes allow the determination of the reduced transition strength of the deexciting $\gamma$-ray transitions, which in turn provide information about the wave functions of the involved nuclear states. In the following sections isomerism in both spherical nuclei (seniority isomers) and deformed nuclei ( $K$ isomers) are discussed.

Nuclei in the vicinity of closed shells often exhibit so-called spin isomerism. In this case excited states are long lived because the available decay paths require high angular-momentum transition and/or low transition energy. For example, the single magic ${ }^{116-130} \mathrm{Sn}$ isotopes are characterized by the existence of a $10^{+}$isomeric state with $v h_{11 / 2}^{2}$ configuration. Due to the short range of the residual interaction, the energy gap between the highest spin $10^{+}$and $8^{+}$states is small, resulting in isomeric states decaying by low energy E2 transitions. The parabolic behavior of the $B\left(E 2 ; 10^{+} \rightarrow 8^{+}\right)$reduced transition strength can be explained by the seniority scheme [347]. Seniority $(v)$ is the number of nucleons which are not 

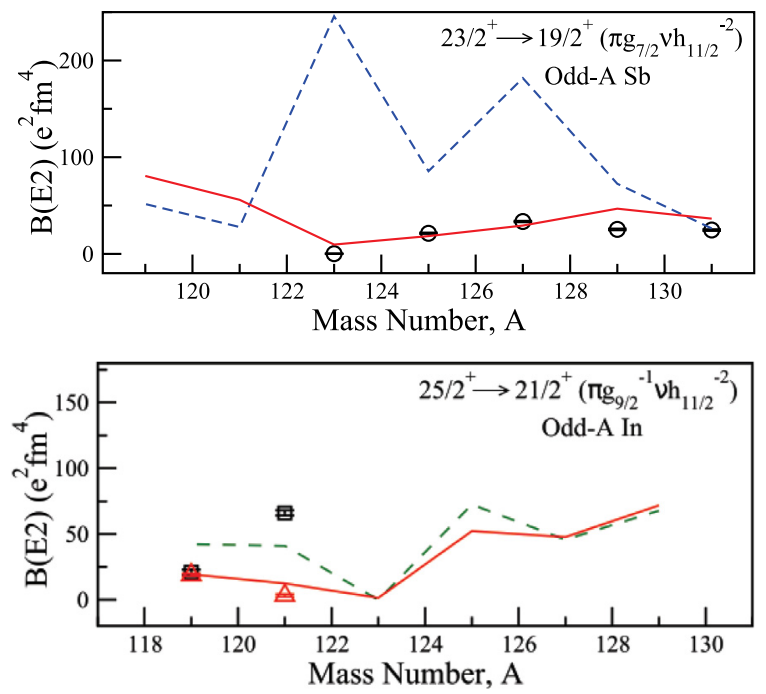

Fig. 71. Top panel Reduced transition strengths $B\left(E 2 ; 23 / 2^{+} \rightarrow 19 / 2^{+}\right)$in odd-mass Sb isotopes. The black symbols are experimental values from [206]. The dotted lines are shell-model calculations using interaction from [348]. The red line shows the values obtained with a modified interaction. Bottom panel Reduced transition strengths $B\left(E 2 ; 25 / 2^{+} \rightarrow 21 / 2^{+}\right)$in odd-mass In isotopes. The new experimental results are shown with red symbols. The shell-model interaction developed for Sb isotopes, shown with green dashed line, provides a good agreement with the data. Further modification so the interaction resulted in the red solid line.

Source: Modified from [206,207].

paired to angular momentum zero. Antimony (Sb) and indium (In) nuclei with an additional proton in the $g_{7 / 2}$ orbital and a proton hole in $g_{9 / 2}$, respectively, exhibit similar isomeric states.

Complementary AGATA studies extended further into the $g d s h$ valence space and isomeric states in the $50 \leq Z, N \leq 82$ region were described in the previous chapter, Section 6.5. Several new findings are also related to the fact that the gdsh valence space is dominated by the high- $j$ intruder $v 0 h_{11 / 2}$ orbital with its large single-particle angular momentum.

Isomeric states were studied in the neutron-rich ${ }^{122-131} \mathrm{Sb} \mathrm{[206]}$ and ${ }^{119-121}$ In [207] isotopes in an experiment at GANIL. They were populated from the fission of a ${ }^{238} \mathrm{U}$ beam with $\mathrm{E} / \mathrm{A}=6.2 \mathrm{MeV}$ on ${ }^{9} \mathrm{Be}$ targets. The fission products were identified unambiguously on ion-by-ion basis using the VAMOS++ spectrometer [30] placed at 20 degrees relative to the beam axis. The prompt $\gamma$ rays were detected with AGATA placed around the target. Delayed $\gamma$ rays depopulating isomeric states were identified by seven EXOGAM HPGe Clover detectors [5] placed at the focal plane of VAMOS. This unique setting allowed to correlate prompt and delayed $\gamma$-ray transitions in the time range of $100 \mathrm{~ns}-200 \mu \mathrm{s}$.

New isomeric states were established in a number of isotopes, both with even and odd mass numbers. Their lifetime and decay properties allowed the determination of reduced transition strengths. These, with the already available data on In, $\mathrm{Sn}$ and Sb isotopes were used to test shell-model interactions. Shell-model interactions previously developed [348] reproduced both excited state energies and transition strengths in $\mathrm{Sn}$ isotopes. On the other hand, in Sb isotopes they reproduced the energy levels, but not the $\mathrm{B}(\mathrm{E} 2)$ pattern of the $23 / 2^{+} \rightarrow 19 / 2^{+}$transitions from the $\pi g_{9 / 2} v h_{11 / 2}^{2}$ predominantly $v=3$ isomeric states. Modification of the available interaction resulted in a good description of the data, as shown in Fig. 71. These modified interaction was further tested by the more recent result on odd-mass In isotopes (lower part of Fig. 71). They slightly overestimate the experimentally obtained new B(E2) values obtained from the decay of the $25 / 2^{+}$isomers. Further adjustment of the interaction between the $g_{9 / 2}$ proton hole and $h_{11 / 2}$ neutron results in an excellent agreement.

These results on the $Z=49 \mathrm{In}$ and $\mathrm{Z}=51 \mathrm{Sb}$ isotopes indicate that the presence of the valence proton (hole in $g_{9 / 2}$ or particle in the $g_{7 / 2}$ ) orbital lead to much more complex neutron wave functions when compared to Sn [206,207]. Consequently, the experimental $B(E 2)$ values do not show the parabolic feature expected for seniority isomers. Their decay pattern is modified by the mixing of seniority $v=3$ and $v=5$ states.

\subsubsection{Conservation of $K$ quantum number from rotational continuum spectra}

In axially-deformed symmetric systems, away from closed shells, $\mathrm{K}$ isomers are the most abundant, $\mathrm{K}$ being the projection of the nuclear angular momentum on the symmetry axis of the nucleus. The isomer existence arises from the approximate conservation of $\mathrm{K}$. In an electromagnetic de-excitation, if $\mathrm{K}$ were a good quantum number, the change in $\mathrm{K}$ should not exceed $\lambda$, the transition multipole order. Transitions that violate this rule are called $\mathrm{K}$ forbidden. In reality, rather than strictly forbidden, such transitions are strongly hindered, resulting in long-lived isomeric states. For the transition rates the reduced hindrance factor quantity is defined as $\mathrm{f}_{v}=\mathrm{F}\left({ }_{w}\right)^{1 / v}$ where $\mathrm{F}_{w}$ is the ratio of the Weisskopf estimate to the measured transition rates and $v=\Delta \mathrm{K}-\lambda$ is the degree of forbiddenness. 

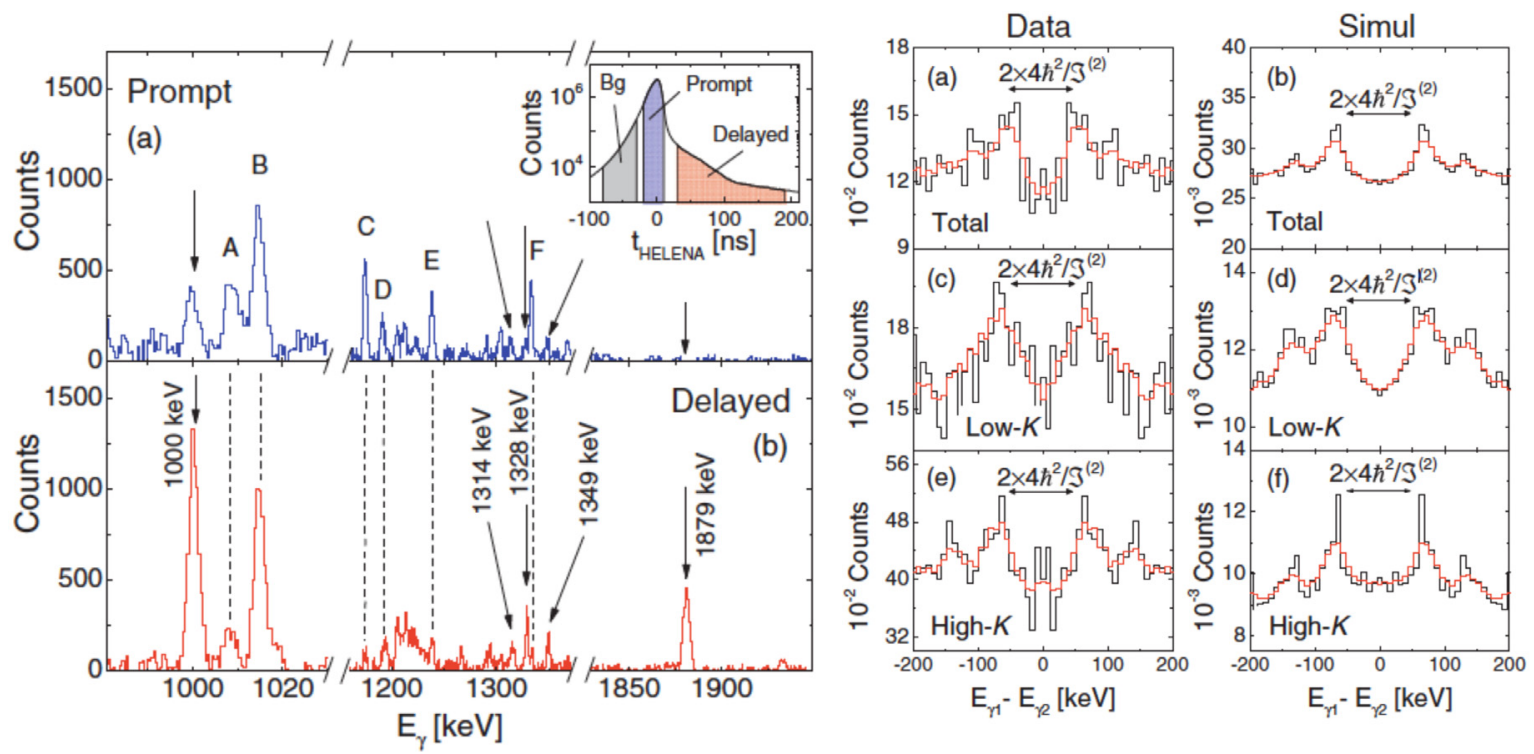

Fig. 72. Left panels: Energy spectra measured with AGATA, associated to prompt (delayed) $\gamma$ decays [panels (a) and (b),respectively]. Arrows indicate transitions depopulating the $12^{+}$and $8^{-}$isomeric states of ${ }^{174} \mathrm{~W}$. Dashed lines point to prompt $\gamma$ transitions of ${ }^{174} \mathrm{~W}$ (indicated with $\mathrm{A}$, C, D, and $\mathrm{E}$ in the top-left panel) or contaminant reaction processes, such as $\gamma$ decay from ${ }^{27} \mathrm{Al}$ and ( $\mathrm{n}, \gamma$ ) on ${ }^{73} \mathrm{Ge}$ (indicated with $\mathrm{B}$ and $\mathrm{F}$ in the top-left panel). The prompt (within $20 \mathrm{~ns}$ ), delayed (30-190 ns), and background ((-80)-(-30)ns) time windows are indicated in the inset (showing a portion of the time spectrum). Right panel: Spectra obtained as a perpendicular cut (60-keV wide) in $\gamma \gamma$ matrices either measured in this experiment (left) or simulated (right). The shown ${ }^{174} \mathrm{~W}$ spectra correspond to an average energy of $820 \mathrm{keV}$. The matrices collect the total $\gamma$-decay flow [panels (a) and (b)] or the $\gamma$ decay in coincidence with low-K [(c) and (d)] or high-K [(e) and (f)] configurations. In the simulation, low-K (high-K) states correspond to $\mathrm{K} \leq 6(\mathrm{~K}>6)$. Arrows indicate the separation between the two innermost ridges. Thin red lines are the first moment $\left(\mu_{1}\right)$ spectra, used in the statistical analysis.

Source: Adapted from Ref. [349].

Empirically, it was found that the associated transition rates depend on the degree of forbiddenness, and for each degree the transition rate is typically slowed down by the so-called reduced hindrance factor equal to $\approx 100$. This empirical rule was at least qualitatively interpreted in terms of the Coriolis mixing with a fixed axial shape. Subsequent studies of $\mathrm{K}$ isomers in the mass region $\mathrm{A} \approx 160-180$ have, however, shown that the reduced hindrance factors $\mathrm{f}_{v}$ can assume remarkably smaller values, as compared to the empirical value. Several direct decays to the $\mathrm{K}=0$ band have been observed. To achieve a more comprehensive picture of the K-mixing phenomenon it is important to gather complementary information from the decay properties of the entire body of discrete excited high-K bands. This approach was employed for the study of ${ }^{163} \mathrm{Eu}$ in which the properties of $\gamma$-decay fluxes feeding low-K and high-K rotational bands were exploited to probe the progressive weakening of selection rules on $\mathrm{K}$ with increasing internal energy $\mathrm{U}$, focusing on the transition between order and chaos in a quantum-mechanical system.

An experiment was performed with the AGATA set up at LNL with the aim to measure and analyze quasi-continuum spectra of $\gamma$ rays emitted at high spins and high internal energy, probing the $\gamma$-decay feeding into specific low-lying configurations in the nucleus ${ }^{174} \mathrm{~W}$. The employed reaction was ${ }^{50} \mathrm{Ti}+{ }^{128} \mathrm{Te}$ at a beam energy of $217 \mathrm{MeV}$ populating ${ }^{174} \mathrm{~W}$ with a relative intensity of the order of $40 \%$. Scintillator $\mathrm{BaF}_{2}$ detectors were used to measure efficiently the time of the $\gamma$-ray emission.

In the left panels of Fig. 72 two $\gamma$-energy spectra measured with AGATA associated to prompt and delayed $\gamma$ decays are displayed in panels (a) and (b), respectively. The arrows indicate transitions depopulating the $12^{+}$and $8^{-}$isomeric states of ${ }^{174} \mathrm{~W}$. Dashed lines point to prompt $\gamma$ transitions of ${ }^{174} \mathrm{~W}$ or contaminant reaction processes. The time gates were then used to put conditions on $\gamma \gamma$ matrices that were successively analyzed using the fluctuation analysis technique described in [353] and applied in several works (see e.g. [354-357]) to study the warm rotation in the continuum. The technique allows to extract information from spectra obtained from diagonal cuts in two dimensional $\gamma \gamma$ matrices that, in case of rotating nuclei, are characterized by a typical ridge valley structure. Some samples of this analysis are shown in the right panels of Fig. 72. Both measurements and simulations are shown, the simulations(on the most right panels) being based on predictions from the cranked-shell model with residual interaction [358] and on the procedure described in [359] and [360]. The analysis for the ${ }^{174} \mathrm{~W}$ nucleus gives a number of excited bands forming the ridge structures which is found to be in good agreement with the predictions.

One relevant issue for the analysis of the rotational ridge structure in the semi-continuum spectra is the dependence on spin of the E2-E1 competition, which has to be determined experimentally by comparing measured intensities of yrast transitions and ridges with the simulated ones. This result for the ${ }^{174} \mathrm{~W}$ nucleus is shown in the left panel of Fig. 73 . With 

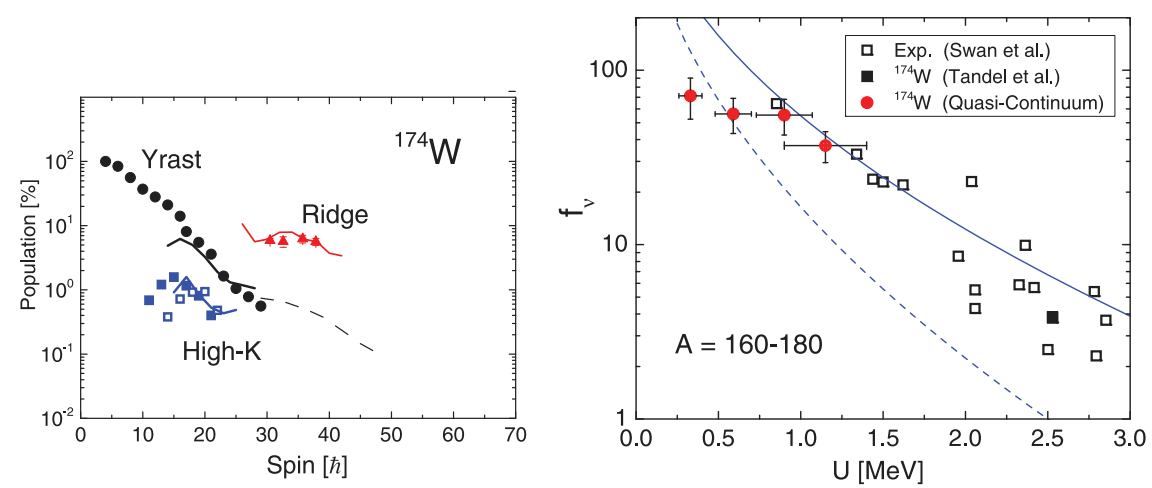

Fig. 73. Left panel: Population intensities, as a function of spins, of the yrast band, high- $\mathrm{K}$ bands $\left(\mathrm{K}=8^{-}, 12^{+}\right)$, and total ridge structures extracted from the analysis of experimental (symbols) and simulated (lines) spectra. In the case of the yrast, the solid and dashed lines represent the contribution of the positive- and negative-parity configurations, respectively. Right panel: Reduced hindrance $\mathrm{f}_{v}$, as a function of internal energy $\mathrm{U}$, for nuclei in the mass region $A \approx 160-180$. Open squares are experimental data from Ref. [350], with the full square representing the reduced hindrance factor reported in Ref. [351] for the $12^{+}$isomer of ${ }^{174} \mathrm{~W}$. Full red circles are obtained from the present quasi-continuum analysis of the quenching of the E1 decay between discrete excited bands of ${ }^{174} \mathrm{~W}$ while the last point at $\mathrm{U}=1.15 \mathrm{MeV}$ refers to the analysis of microscopic levels extending into the damping region. The solid (dotted) line represents the $\mathrm{f}_{v}$ curve for $v=4(v=3)$, calculated with the analytical model of Ref. [352].

Source: Adapted from Ref. [349].

the ensemble of information deduced by this analysis of the different parts of the phase space available for the $\gamma$-ray emission leading to the isomers and to the prompt states, it was possible to obtain the average reduced hindrance which is related to the E1 transition probability T(E1) needed to reproduce the data in left panel of Fig. 73. The obtained values from the quasi continuum analysis are compared with the results from discrete transitions from Refs. [361] and [350]. The solid (dotted) line represents the $\mathrm{f}_{v}$ curve for $v=4(v=3)$, calculated with the analytical model of Ref. [352].

The AGATA experiment on the ${ }^{174} \mathrm{~W}$ nucleus, which has isomeric states due to the presence of high-K configurations, suggests that a K-mixing process between low-K and high-K rotational bands due to temperature effects plays an important role already at rather low excitation energy. The experiment and the global analysis of decay properties of the entire body of discrete excited bands have allowed to probe the onset region of band mixing. This represents a step forward in the understanding of the basic rules governing the isomeric systems and calls for similar, additional investigation at higher excitation energies, where the erosion of the $\mathrm{K}$ quantum number becomes even more severe.

\section{The $\gamma$-ray detection to investigate reaction mechanisms and nucleonic degrees of freedom}

The detection of gamma-rays is a powerful tool not only to investigate nuclear structure but also to understand reaction mechanisms at play for different bombarding energy regimes, namely from energies around the Coulomb barrier up to hundreds AMeV. A few experiments carried out with AGATA have addressed open questions in the understanding of reactions among heavy ions, since the gamma-ray emission from the products can shed light on the complex processes occurring during the collisions. Here we discuss the results obtained in connection with three specific problems: (i) the transfer of one- and two-neutrons at the Coulomb barrier; (ii) the population of isomeric states in heavy ion collision up to $1 \mathrm{AGeV}$; (iii) the excitation on nucleonic degree of freedom at bombarding energy in the pion production region.

\subsection{The $\gamma$ decay to probe reaction mechanisms at the Coulomb barrier}

Low-energy multi-nucleon transfer reactions are among the most important tools to probe nucleon-nucleon correlations in nuclear systems, which are particularly relevant in the studies of neutron-rich nuclei where they play an important role in stabilizing the system by increasing the binding energy. For the description of the reaction around the barrier it was found that the evolution of a heavy-ion collision is dominated by the structure properties of the reactants, in particular by their surface and single-particle degrees of freedom, but the role of nucleon-nucleon correlations, as expressed by the transfer of pairs, is still an open question (see [362] and references therein).

From an experiment, using the ${ }^{60} \mathrm{Ni}+{ }^{116} \mathrm{Sn}$ reaction, made to investigate ground-state correlations via the cross section for the transfer probabilities for one- and two-neutrons, very interesting results were found for the two neutron channel [364]. This reaction, at the employed bombarding energy of $245 \mathrm{MeV}$, was chosen because this system is very well Q-value matched for the ground-state to ground-state transition; this matching condition is relevant because particleparticle correlations are essential in defining the ground-state properties. The transfer probabilities, measured up to very large distances of closest approach, were analyzed by employing a microscopic theory of [365], which, for the first time in a heavy ion collision, provided a consistent description of one- and two-neutron transfer channels, in shape and magnitude. The ground-state to ground-state transition was calculated by describing the ground states of ${ }^{60} \mathrm{Ni}$ and ${ }^{114} \mathrm{Sn}$ in the BCS approximation with a standard state-independent pairing force. 


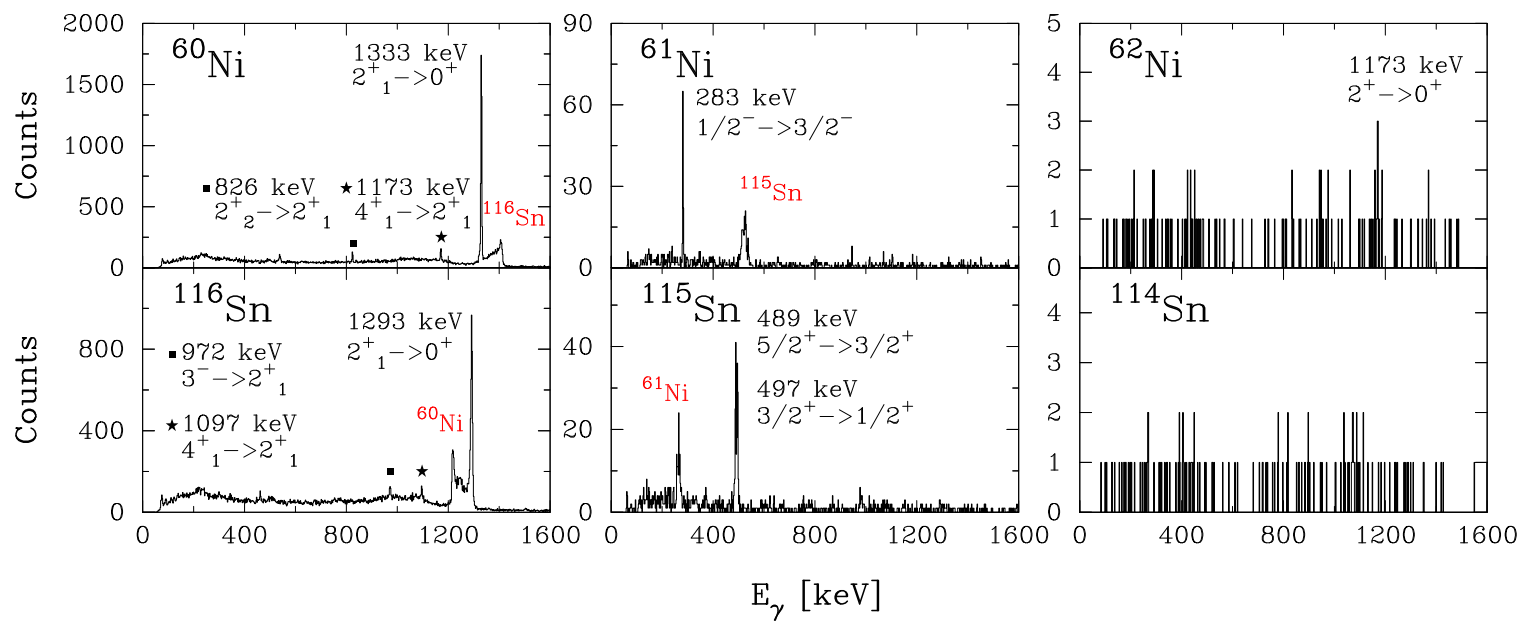

Fig. 74. Top panels: Doppler-corrected $\gamma$ spectra measured with AGATA for ${ }^{60} \mathrm{Ni},{ }^{61} \mathrm{Ni}$, and ${ }^{62} \mathrm{Ni}$ detected in PRISMA. Bottom panel: Doppler-corrected spectra for the heavy binary partners ${ }^{116} \mathrm{Sn},{ }^{115} \mathrm{Sn}$, and ${ }^{114} \mathrm{Sn}$ (the bin is $2 \mathrm{keV}$ wide). The strongest transitions are labeled with the $\gamma$-ray energy and the spin and parity of the initial and final states. The broader peaks, corresponding to the wrongly Doppler-corrected reaction partner, are also labeled (red color) in each frame.

Source: Adapted from Ref. [363].

One important aspect to be checked in order to corroborate the conclusion that the two-neutron transfer process involves only the ground states is to exclude excitations in ${ }^{62} \mathrm{Ni}$. The measured energy distribution for the two-neutron transfer channel was found to be concentrated in an energy region with a width of $\sim 2 \mathrm{MeV}$, while the energy of the $2^{+}$state in ${ }^{62} \mathrm{Ni}$ is at $1.173 \mathrm{MeV}$ and thus the contribution of the excitation of states in the two-neutron transfer channel could not be excluded. Therefore a measurement using also the AGATA array was made to search for possible excitations.

The $\gamma$-ray spectra measured with AGATA when ${ }^{60} \mathrm{Ni},{ }^{61} \mathrm{Ni}$, and ${ }^{62} \mathrm{Ni}$ ions are detected in PRISMA are shown in Fig. 74. For the ${ }^{62} \mathrm{Ni}$ only few counts were identified in the transition from the $2^{+}$state. From the experimental intensities of the $\gamma$ lines the strength for the two-neutron transfer channel were obtained. This turned out to be less than $24 \%$ and is compatible with the theoretical description of the transfer process that predicted a dominance of the ground-state to ground-state transition. The measurement provided values of the angular distribution of the cross section in the interval $\theta_{l a b}=65^{\circ}-75^{\circ}$; thus following the procedure in [365] the $\mathrm{P}_{t r}$, the distance of the closest approach $\mathrm{D}$, was extracted for the $1 \mathrm{n}$ and $2 \mathrm{n}$ channels. These data are shown with open symbols in Fig. 75 in comparison with other previous data and the predictions. The predicted values of the angular distribution of the cross section for the different channels as obtained with coupled-channel calculations are shown in the left panel of Fig. 75.

The result obtained with AGATA on the population of the $2^{+}$state in ${ }^{62} \mathrm{Ni}$ are supported also by other experiments; in fact, the population of the different states in the same nucleus was studied via two-neutron transfer by employing $(\mathrm{t}, \mathrm{p})$ and $(\mathrm{p}, \mathrm{t})$ reactions. For the ${ }^{60} \mathrm{Ni}^{1116} \mathrm{Sn}$ reaction it was found that the population of the low-lying $2^{+}$state is much smaller than the one of the ground state (the ratio being of a factor of 10 to 3 ). Also the light-ion $\left({ }^{14} \mathrm{C},{ }^{12} \mathrm{C}\right)$ reaction was used to study the same nucleus and the population of the $2^{+}$state was only a factor of 2 smaller than that of the ground state. This could indicate that with heavier projectiles the multi-step mechanism (inelastic followed by transfer and vice versa) and the transfer of angular momentum play important roles in the transfer mechanism. It is worth noting that in Ref. [366] the analysis of the two-neutron transfer reactions incorporated both successive and simultaneous contributions and showed that the successive one greatly dominated the reaction.

\subsection{Reactions and isomeric ratios}

The presence of long-lived states can be used as a tool to increase the sensitivity of experiments. The time before the isomer decays can be used to transport nuclei produced with very low yields, and their decay studied far from the production target. One of the applications of this technique is the study of the reaction mechanism of high-energy fragmentation. Decay of spin isomers were observed in even-even nuclei produced from a $E / A=1 G e V{ }^{208} \mathrm{~Pb}$ beam impinging on a ${ }^{9} \mathrm{Be}$ target in an experiment performed at GSI as part of the PRESPEC campaign. The nuclei of interest were identified and separated by the fragment separator FRS, and then implanted into a passive stopper. AGATA was used to detect the isomeric decays from isomeric states in ${ }^{198,200,202,206} \mathrm{~Pb}[367]$ and in ${ }^{206} \mathrm{Hg}[52,367]$.

The extracted isomeric ratio, defined as the fraction of those produced in an isomeric state for a given nuclide, are important indicators of the reaction mechanism. Fragmentation reactions are considered two-step reactions; with a first abrasion phase removing nucleons and resulting in a highly excited pre-fragment. In the second ablation phase the 

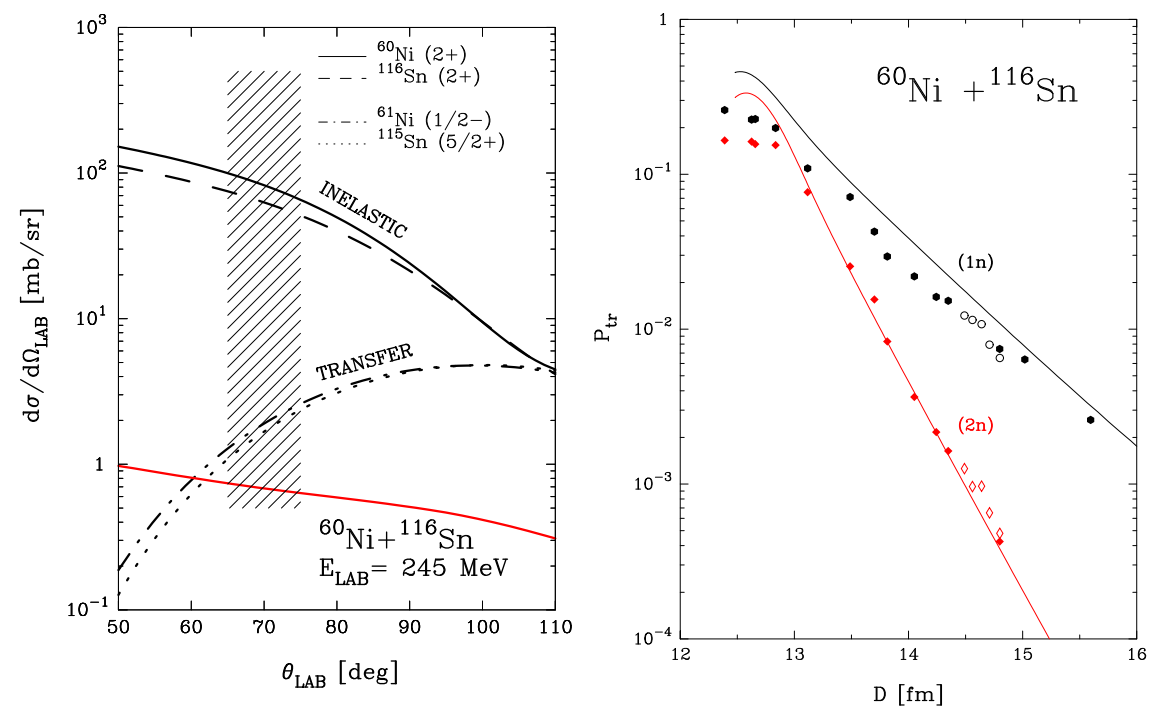

Fig. 75. Left panel: Differential cross sections, from coupled-channels or DWBA calculations, for the indicated states in ${ }^{60,61} \mathrm{Ni}$ and ${ }^{116,115} \mathrm{Sn}$ in the ${ }^{60} \mathrm{Ni}^{116} \mathrm{Sn}$ reaction at $\mathrm{E}_{\text {lab }}=245 \mathrm{MeV}$. The red (gray) line is the ratio of the calculated elastic cross section with nuclear and Coulomb contributions over the predicted value of the Rutherford cross section (only Coulomb). The hatched area corresponds to the PRISMA angular acceptance. Right panel: Experimental (symbols) and microscopically calculated (lines) transfer probabilities for the one- and two-neutron pickup channels plotted as a function of the distance of closest approach D. Open symbols correspond to the presently obtained angular distribution in direct kinematics while solid symbols refer to the excitation function previously performed in inverse kinematics.

Source: Adapted from Ref. [363].

pre-fragment emits nucleons until the excitation energy is below the nucleon evaporation energy, resulting in the final fragment. The angular-momentum population is determined mainly by the first phase.

${ }^{206} \mathrm{Hg}$ nuclei are produced by direct removal of two protons from ${ }^{208} \mathrm{~Pb}$. The contribution of second step in this case (one-proton removal followed by one-proton evaporation) is minimal when producing neutron-rich nuclei. Therefore the isomeric ratios can be calculated using information on the structure of the states in ${ }^{206} \mathrm{Hg}$ and nucleon-removal reaction theory [368]. In addition in such cases the isomeric ratio has a strong dependence on the longitudinal momentum of the fragment which can be observed [369]. In contrast, ${ }^{206} \mathrm{~Pb}$ can be populated both in single-step and two-step processes. The aforementioned calculations describe reasonably the population of both the higher-lying $12^{+}$isomer which should be populated directly, and the lower-lying $7^{-}$which is partly produced via removal of a single neutron followed by neutron evaporation [367]. Calculations of the population of isomeric states in nuclei further in mass from the fragment presently cannot be performed by considering the properties of the individual states, and rely on statistical treatment.

Knowledge of the isomeric composition of the beam is also essential for the interpretation of secondary reaction (Coulomb or nucleon-removal) experiments, as in these only long lived states can be involved (excited or nucleons removed from).

\subsection{Nucleonic degrees of freedom}

In nuclear physics, protons and neutrons are usually considered elementary particles, and consequently both proton and neutron numbers are conserved. However, neither of them are fundamental particles and they have a quark substructure. Their lowest energy excitation is the $\Delta$ resonance at $1232 \mathrm{MeV}$, only few hundreds of $\mathrm{MeV}$ higher than the groundstate-mass energy at $\sim 939 \mathrm{MeV}$. Consequently, in relativistic-energy fragmentation reactions, this internal structure can play a part. The first evidences are from the 1980s, when D. Bachelier et al. reported the "First observation of the $\Delta^{+}$resonance in relativistic heavy ion charge-exchange reactions" [370]

The first case proving that the internal structure of the nucleons affects the population of individual excited states in a nucleus were reported in [57]. In the experiment performed at GSI, an $E / A=500 \mathrm{MeV}{ }^{56} \mathrm{Fe}$ beam impinged on a ${ }^{9} \mathrm{Be}$ target. ${ }^{54} \mathrm{Fe}$ was selected to be transmitted by the fragment separator and subsequently implanted in a passive stopper. The decay of its $10^{+}$isomeric state with a lifetime of $T_{1 / 2}=364(7)$ ns [371] was detected with AGATA, placed $15 \mathrm{~cm}$ downstream from the nominal center of AGATA in order to increase detection efficiency [28].

Without considering the internal structure of nucleons in the ${ }^{56} \mathrm{Fe} \rightarrow{ }^{54} \mathrm{Fe}$ reactions only two neutron-states can be populated. Consequently, the maximum spin generated is 6 and it corresponds to the $v f_{7 / 2}^{-2}$ configuration. In contrast, the configuration of the $10^{+}$isomeric state requires at least four unpaired nucleons. Nevertheless, the decay of the $10^{+}$isomeric state was observed. The determined isomeric ratio as a function of the momentum transfer of the ${ }^{54} \mathrm{Fe}$ nuclei is shown in Fig. 76 (the parallel momentum is determined from the measured position of individual ${ }^{54} \mathrm{Fe}$ nuclei 


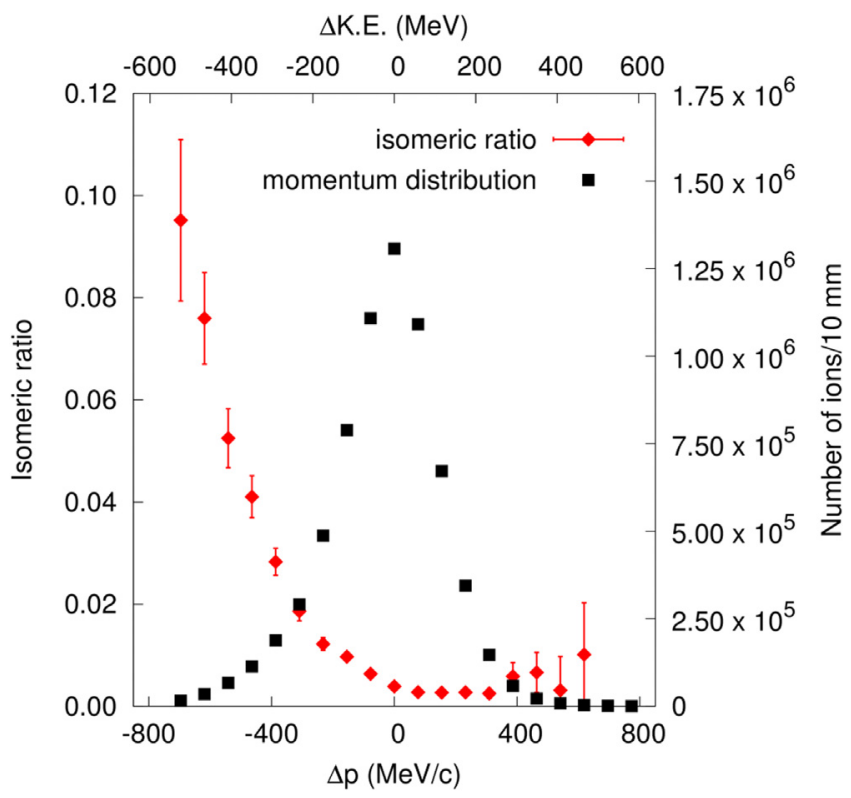

Fig. 76. Isomeric ratio of the $10^{+}$isomeric state and the parallel momentum distribution of the ${ }^{54} \mathrm{Fe}$ nuclei, shown as function of momentum transfer (lower $\mathrm{X}$ axis) and kinetic energy loss (upper $\mathrm{X}$ axis).

Source: Taken from [57].

at the dispersive focal plane in the middle of the fragment separator). The isomer is produced predominantly in the low-momentum tail, in striking contrast to the symmetric distribution normally observed in fragmentation reactions. The parallel momentum distribution is also shown in Fig. 76; it is slightly asymmetric, exhibiting a small tail at the low-momentum side (for details see Ref. [372]).

The two experimental observations: the low-momentum tail of the momentum distribution and the predominant production of the isomer in this tail corresponding to $\sim 300 \mathrm{MeV}$ energy shift, indicates that the $10^{+}$isomeric state in ${ }^{54} \mathrm{Fe}$ is populated due to the internal excitation of nucleons. For example, one of the protons of the ${ }^{56} \mathrm{Fe}$ beam can be excited into the $\Delta^{+}$resonance, which decays into a neutron emitting a positive pion (which takes away energy), a process which leads to two unpaired particles. In addition, two neutron are also removed from ${ }^{56} \mathrm{Fe}$, creating additional two unpaired nucleons. Therefore, four-particle states can be populated in this process. The existence of a large number of isomeric states allows the extension of the presented work to other regions of the chart of nuclides, providing information on the role of nucleonic excitations in high-energy heavy-ion collisions.

\section{Conclusions and outlook}

This review presents the comprehensive amount of results produced so far with the AGATA array in the first phases of its realization and exploitation (see Table 3). It provides evidence that $\gamma$-ray spectroscopy continues to be, and it will be even more during upcoming years, an indispensable tool for the study of nuclei and of the different arrangements of nucleons within them. The key observables, which are mandatory to guide our understanding of nuclear structure, namely excitation energies, lifetimes and spins, were determined with the AGATA setups in various nuclei after they have been prepared in different specific configurations. The experimental conditions allowing to reach nuclei and nuclear configurations of interest were such that the selectivity, efficiency and position sensitivity of the $\gamma$-ray detectors were essential for the success of the studies. The new concept underlying a $\gamma$-ray tracking array was successfully implanted and deployed to identify the position and energy of $\gamma$-ray interaction points. It was proved to be crucial in combating the Doppler broadening particularly to measure the energies of $\gamma$-rays emitted from in flight nuclei with high velocities exceeding $20 \%$ of the speed of light as in the fragmentation reactions. The experiments performed so far used a good variety of available beams, primarily heavy ions. For the identification of the $\gamma$-ray emitting nuclei after nuclear reactions providing many products simultaneously an excellent exploitation was made of the channel selection features of the magnetic spectrometers available at the different laboratories.

This summary section recalls and lists the main results of the experiments performed with AGATA up to now. The same sequence of subjects, that was used to organize the content in this review paper, is also adopted for the following conclusive statements. 
Table 3

List of nuclei (element and isotopes given in the first two columns) investigated with the AGATA experiments discussed in this review. The measured quantities and the sections are given in columns 3 and 4. References to pertinent AGATA publications are in column 5.

\begin{tabular}{|c|c|c|c|c|}
\hline Element & Isotopes & Measured quantities & Sections & \\
\hline $\mathrm{O}$ & 19,20 & Levels and transition probabilities & 3 & [72] \\
\hline K & 40 & Levels & 6.2 & {$[227]$} \\
\hline $\mathrm{Ca}$ & 42 & Levels and transition probabilities & 6.1 & [217-219] \\
\hline $\mathrm{Ti}$ & 46 & Levels and transition probabilities & 7.1.1 & [54] \\
\hline $\mathrm{Ti}$ & 52,54 & Level lifetimes and transition probabilities & 4.1 & {$[106]$} \\
\hline V & 46 & Levels and transition probabilities & 7.1.1 & {$[54]$} \\
\hline $\mathrm{Cr}$ & 46 & Levels and transition probabilities & 7.1 .1 & {$[54]$} \\
\hline $\mathrm{Fe}$ & 54 & Isomeric ratio and momentum distribution & 8.3 & [57] \\
\hline $\mathrm{Fe}$ & 62,64 & Levels and lifetimes & 5.1 .3 & [164] \\
\hline $\mathrm{Fe}$ & 62,64 & High lying continuum & 5.2 .3 & {$[55]$} \\
\hline Co & $61,63,65$ & Levels and lifetimes & 5.1 .1 & {$[163,164]$} \\
\hline $\mathrm{Cu}$ & $69,71,73$ & Levels and lifetimes & 5.1 .1 & {$[162]$} \\
\hline $\mathrm{Zn}$ & $70,72,74$ & Level lifetimes and transition probabilities & 4.2 & [116] \\
\hline $\mathrm{Ga}$ & 81 & $\gamma$-ray spectroscopy & 4.3 .3 & [143] \\
\hline $\mathrm{Ge}$ & 84 & Levels and transition probabilities & 7.2 .1 & [339] \\
\hline Se & $80,82,84,86$ & Level lifetimes and transition probabilities & 4.3.1 & {$[133,134]$} \\
\hline Se & 85 & Level lifetimes & 4.3.2 & {$[141]$} \\
\hline Se & 86 & Levels and transition probabilities & 7.2 .1 & {$[339,340]$} \\
\hline $\mathrm{Kr}$ & 87 & Level lifetimes & 4.3.2 & [141] \\
\hline $\mathrm{Kr}$ & 88 & Levels and transition probabilities & 7.2 .1 & {$[339,340]$} \\
\hline $\mathrm{Kr}$ & 96 & Levels & 6.3 & [235] \\
\hline $\mathrm{Zr}$ & 80 & GDR decay in CN nucleus & 7.1.2 & {$[195,196]$} \\
\hline $\mathrm{Rb}$ & 81 & GDR decay in CN nucleus & 7.1.2 & {$[195,196]$} \\
\hline $\mathrm{Ru}$ & 88 & Levels & 7.1 .3 & [336] \\
\hline $\mathrm{Zr}$ & 90 & High-lying levels and population cross section & 5.2 .2 & [179] \\
\hline In & 119,121 & Isomeric states and transition probabilities & 7.3 & [207] \\
\hline Sn & 106,108 & Level lifetimes and transition probabilities & 4.4 & {$[145]$} \\
\hline Sn & 124 & High-lying levels and population cross section & $5.2 .2,5.2 .4$ & {$[178,191]$} \\
\hline $\mathrm{Sb}$ & $122-131$ & Isomeric states and transition probabilities & 7.3 & [206] \\
\hline $\mathrm{Xe}$ & $131-135$ & Levels and isomeric states & $6.5,6.6$ & [255-258] \\
\hline $\mathrm{Ba}$ & $133-137$ & Levels and isomeric states & $6.5,6.6$ & {$[256,259-261]$} \\
\hline $\mathrm{Ba}$ & 140 & Level population probabilities & 7.2 .2 & [345] \\
\hline $\mathrm{Ce}$ & 140 & High-lying levels and population cross section & 5.2 & [181] \\
\hline W & 174 & Rotational continuum & 7.3 .1 & [349] \\
\hline Os & 196 & Levels & 6.7 & [312] \\
\hline Pt & 200 & $\gamma$-ray spectroscopy & $5.1 .2,6.7$ & {$[240]$} \\
\hline $\mathrm{Hg}$ & 206 & Isomeric ratio & 8.2 & {$[52,367]$} \\
\hline $\mathrm{Pb}$ & $198,200,202,206$ & Isomeric ratio & 8.2 & [367] \\
\hline $\mathrm{Pb}$ & 207 & Levels and lifetimes & 5.1 .2 & [169] \\
\hline $\mathrm{Pb}$ & 208 & High-lying levels and population cross section & 5.2 & [177] \\
\hline $\mathrm{U}$ & 240 & Levels & 6.7 & [315] \\
\hline
\end{tabular}

- "Interactions"- Electric and magnetic-decays from low-lying states in light neutron-rich nuclei of elements such as C and $\mathrm{O}$ were found to provide a demanding test of theoretical $\mathrm{ab}$ initio approaches and to be selective in the role of $3 \mathrm{~N}$ forces. Challenging lifetime measurements have to be pursued also in the future.

- "Single-particle excitations and new shells"- Confirmation of subshell closure at N=32 is found with the measurements for neutron-rich Ti nuclei. The spectroscopy for the ${ }^{81} \mathrm{Ga} \mathrm{N}=50$ isotone supports the doubly magic character of ${ }^{78} \mathrm{Ni}$ and for $\mathrm{N}>50$ Se isotopes suggests the development of structures with triaxial character. The spectroscopy of neutron-deficient $\mathrm{Sn}$ isotopes has evidenced a strong interplay between the pairing strength and quadrupole correlations.

- "Collective excitations and coupling to single particles"- A number of the low-lying dipole states in ${ }^{90} \mathrm{Zr},{ }^{124} \mathrm{Sn},{ }^{140} \mathrm{Ce}$ and ${ }^{208} \mathrm{~Pb}$ nuclei were found to have an isoscalar character. Several high-lying discrete states of quadrupole type in ${ }^{124} \mathrm{Sn}$ were interpreted as neutron-skin excitations. Some particle-core vibrations of quadrupole and octupole type were found in some of the even-odd nuclei around the neutron-rich Ni isotopes. In general, the coupling of the odd nucleon to the core are rather complex. An interesting coupling of one hole to a two-octupole-phonon excitation was identified in ${ }^{207} \mathrm{~Pb}$.

- "Shapes and high spins"- Highly-deformed configurations were seen for the first time in ${ }^{42}$ Ca after Coulomb excitation. The new excitation mechanism together with advanced analysis techniques yielded precise and partially even model-independent observables related to quadrupole deformation and triaxiality. A weakly deformed groundstate band and a highly-deformed slightly triaxial side band establish unambiguously the general picture of shape coexistence in ${ }^{42} \mathrm{Ca}$. The nuclear structure north west of $\mathrm{A}=132$ was addressed with many more details as compared to previous fission-fragment measurements by employing also MNT reaction products. Isomerism and high-spin properties like backbending were inferred form detailed spectroscopy. Especially, collective behavior is 


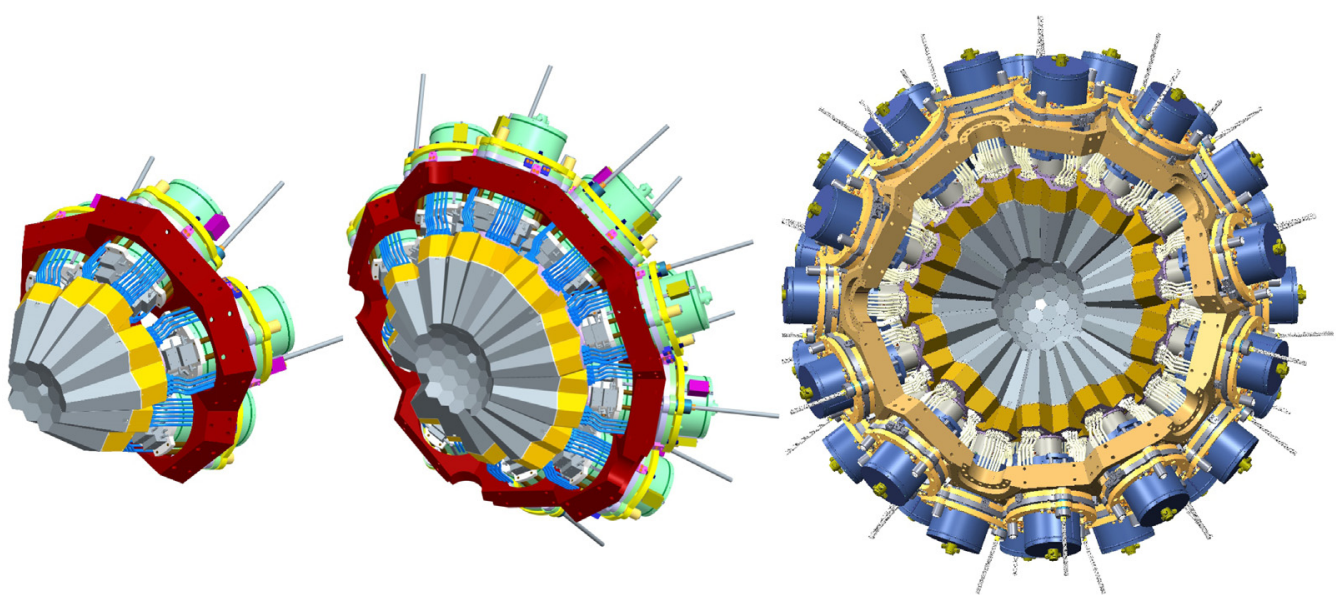

Fig. 77. Picture of the AGATA array at various states of completion. Left panel: The demonstrator configuration with five ATCs was employed in the first experimental campaign at LNL. Middle panel: The detector configuration with 45 HPGe detectors in 15 ATCs resembles the detector configuration during the GANIL campaign. Right panel: The full $4 \pi$ configuration will consist of 60 cluster modules. (Pictures courtesy of UKRI-STFC.).

successfully confronted with microscopical theoretical calculations tracing back the orbital specific deformations and the underlying nucleon-nucleon residual interactions.

- "Symmetries in nuclei"- Isospin symmetry was investigated in the $\mathrm{A}=46{ }^{46} \mathrm{Cr}-{ }^{46} \mathrm{~V}-{ }^{46} \mathrm{Ti}$ isobar multiplet and at high excitation energy for $\mathrm{N}=\mathrm{Z}=40$ so that details of the Coulomb effects in the energies and wave-function were deduced. The $T=0$ neutron-proton pairing was probed by establishing the yrast structure of the $\mathrm{N}=\mathrm{Z}$ nucleus ${ }^{88} \mathrm{Ru}$. The concept of pseudospin symmetry was invoked to explain the unexpectedly high collectivity in ${ }^{84} \mathrm{Ge}$. An $\alpha$-transfer reaction was used to test the population of mixed symmetry states in ${ }^{140} \mathrm{Ba}$. Advances in the understanding of complex configurations giving rise to isomerism were made for neutron-rich In and Sb isotopes.

- "Reaction mechanisms and nucleonic degrees of freedom" Evidence that reactions at energy around the Coulomb barrier populate quadrupole states with a two-step process was found. Isomeric ratios were used to probe the reaction mechanism of relativistic-energy fragmentation. The two-neutron knock out reactions at $\mathrm{E} / \mathrm{A}=500 \mathrm{MeV}$ of ${ }^{56} \mathrm{Fe}$ showed that the population of a specific isomeric state is due to the excitation of the $\Delta$ nucleon resonance.

It has to be noted that a large fraction of these results call for future investigations. Therefore, many of these studies represent good starting points for the definition of future directions and plans.

The future of AGATA is resting on the same two pillars which characterized its past: (i) The gradual increase in the number of detectors with the aim to reach its final $4 \pi$ configuration. (ii) The continuous exploitation of the available device in order to maximize the science output. The future experiments will benefit especially by employing a variety of beams provided by different european accelerator laboratories. The completion of AGATA is one of the highest priorities of the European nuclear physics community [373]. The number of triple segmented cluster detectors (ATC) has continuously grown from the demonstrator configuration with five ATCs and the final goal is to reach up to 60 ATCs as part of the AGATA Memorandum of Understanding. In 2020 the detector configuration included already more ATCs than the detector configuration shown in the middle panel of Fig. 77. A major effort is ongoing by the AGATA collaboration to continually evolve the spectrometer by adding new ATCs.

It is envisaged that AGATA will return to LNL, GSI/FAIR and GANIL. These facilities are undergoing dramatic developments, offering new opportunities in the future. Within the approved timeline AGATA will return to LNL in Italy, starting operation there in 2022, where it will take advantage of both stable and radioactive beams at around the Coulomb barrier energies. Initially, experiments will profit from newly developed heavy beams as well as from a large range of ancillary detectors. In the second phase the new SPES facility [374] will provide radioactive beams. Neutron-rich fission fragments will be produced from an uranium(-carbide) target by a high-intensity $40 \mathrm{MeV}$ proton beam. The nuclei of interest will be mass separated and re-accelerated using the existing accelerators. At the GSI site, the Facility for Antiproton and Ion Research (FAIR) [375] is under construction. The new SIS-100 synchrotron will allow higher primary-beam intensities. The future SUPER Fragment Separator [376], where SUPER refers to the use of superconducting magnets, will have higher transmission for fragmentation and fission products compared to the existing FRS and will provide cleaner beams due to the higher number of dipole magnets. AGATA will return there for further HISPEC $[53,377]$ (high energy spectroscopy) campaigns after the low-energy branch of the SuperFRS is commissioned. At GANIL in addition to the high-intensity stable beams up to uranium with energies up to $E / A=100 \mathrm{MeV}$, radioactive beams will be also available. These could be intermediate energy fragmentation beams or re-accelerated Coulomb energy beams of the SPIRAL1 ISOL facility. In the long term new opportunities will be provided by the use of re-accelerated beams related to the SPIRAL2 [378] developments. 
Other facilities interested to host AGATA are the ISOLDE facility at CERN and the heavy ion accelerator laboratory at the Jyväskylä. At ISOLDE the new HIE-ISOLDE post-accelerator [379] would provide radioactive beams with energies $E / A<10 \mathrm{MeV}$ produced in spallation targets by $1.4 \mathrm{GeV}$ protons. In Finland, AGATA could use high-intensity stable beams, as well as the capabilities offered by coupling to both vacuum mode and gas-filled recoil separators.

In conclusions, in the past decade the traveling AGATA tracking array enabled novel experiments addressing topical nuclear physics questions. The science case for future deployments of the array [4], based on unique strengths offered by individual facilities, was also developed.

\section{Acknowledgments}

The long and fruitful collaboration within the AGATA collaboration is gratefully acknowledged by the authors. The authors thank F. Didierjean (IPHC) for his valuable contribution in improving the figure quality.

\section{References}

[1] S. Akkoyun, et al., Nucl. Instrum. Methods Phys. Res. A 668 (2012) 26

[2] AGATA publication list; https://www.agata.org/.

[3] GRETINA publication list; http://gretina.lbl.gov/publications.

[4] W. Korten, et al., Eur. Phys. J. A 56 (2000) 137.

[5] J. Simpson, et al., Act. Phys. Hung. Ser. Heavy Ion Phys. 11 (2000) 159.

[6] S. Shimoura, et al., Nucl. Instrum. Methods Phys. Res. A 525 (2004) 188.

[7] N. Warr, et al., Eur. Phys. J. A 49 (2013) 40

[8] W.F. Mueller, et al., Nucl. Instrum. Methods Phys. Res. A 466 (2001) 492

[9] H.C. Scraggs, et al., Nucl. Instrum. Methods Phys. Res. A 543 (2005) 431.

[10] M.A. Deleplanque, et al., Nucl. Instrum. Methods Phys. Res. A 430 (1999) 292.

[11] K. Vetter, et al., Nucl. Instrum. Methods Phys. Res. A 452 (2000) 105.

[12] I.Y. Lee, M.A. Deleplanque, K. Vetter, Rep. Progr. Phys. 66 (2003) 1095.

[13] Th. Kröll, D. Bazzacco, Nucl. Instrum. Methods Phys. Res. A 463 (2001) 227.

[14] A. Lopez-Martens, K. Hauschild, A. Korichi, J. Roccaz, J.-P. Thibaud, Nucl. Instrum. Methods Phys. Res. A 533 (2004) 454.

[15] A. Wiens, et al., Nucl. Instrum. Methods Phys. Res. A 618 (2010) 223.

[16] G. Pascovici, et al., WSEAS Trans. Circ. Sys. 7 (2008) 470.

[17] A. Pullia, et al., IEEE Symp. Conf. Rec. Nucl. 3 (2004) 1411.

[18] B. Bruyneel, P. Reiter, G. Pascovici, Nucl. Instrum. Methods Phys. Res. A 569 (2006) 764.

[19] B. Bruyneel, P. Reiter, G. Pascovici, et al., Nucl. Instrum. Methods Phys. Res. A 569 (2006) 774

[20] B. Bruyneel, et al., Nucl. Instrum. Methods Phys. Res. A 599 (2009) 196.

[21] B. Bruyneel, et al., Nucl. Instrum. Methods Phys. Res. A 608 (2009) 99.

[22] A. Wiens, et al., Eur. Phys. J. A 49 (2013) 47.

[23] X. Grave, et al., 14th IEEE-NPSS Real Time Conference, 2005, p. 5.

[24] R. Venturelli, D. Bazzacco, LNL Annual Report 2004, 2005, p. 220.

[25] B. Bruyneel, B. Birkenbach, P. Reiter, Eur. Phys. J. A 52 (2016) 1.

[26] F. Recchia, et al., Nucl. Instrum. Methods Phys. Res. A 604 (2009) 555.

[27] P.-A. Söderström, et al., Nucl. Instrum. Methods Phys. Res. A 638 (2011) 96.

[28] N. Lalovic, et al., Nucl. Instrum. Methods Phys. Res. A 806 (2016) 258.

[29] A.M. Stefanini, et al., Nucl. Phys. A 701 (2002) 217

[30] M. Rejmund, et al., Nucl. Instrum. Methods Phys. Res. A 646 (2011) 184.

[31] Ch. Schmitt, et al., Nucl. Instrum. Methods Phys. Res. A 621 (2010) 558.

[32] P.G. Bizzeti, et al., Eur. Phys. J. A 51 (2015) 49.

[33] T. Lauritsen, et al., Nucl. Instrum. Methods Phys. Res. A 836 (2016) 46.

[34] A. Gadea, et al., Instrum. Methods Phys. Res. A 654 (2011) 88.

[35] F.C.L. Crespi, et al., Nucl. Instrum. Methods Phys. Res. A 705 (2013) 47.

[36] A. Bracco, F. Camera, F.C.L. Crespi, B. Million, O. Wieland, Eur. Phys. J. A 55 (2019) 233.

[37] A. Vogt, et al., Phys. Rev. C 92 (2015) 024619.

[38] C.H. Dasso, G. Pollarolo, A. Winther, Phys. Rev. Lett. 73 (1994) 1907.

[39] J. Valiente-Dobonm, et al., AIP Conf. Proc. 853 (2006) 202.

[40] A. Atac, et al., Nucl. Instrum. Methods Phys. Res. A 607 (2009) 554

[41] J. Ljungvall, J. Nyberg, Nucl. Instrum. Methods Phys. Res. A 550 (2005) 379.

[42] H. Geissel, et al., Nucl. Instrum. Methods Phys. Res. B 70 (1992) 286.

[43] P. Golubev, et al., Nucl. Instrum. Methods Phys. Res. A 723 (2013) 55.

[44] A. Giaz, et al., Nucl. Instrum. Methods Phys. Res. A 721 (2013) 910.

[45] H.-J. Wollersheim, et al., Nucl. Instrum. Methods Phys. Res. A 537 (2005) 637.

[46] K. Moschner, et al., Phys. Rev. C 94 (2016) 054323.

[47] G. Guastalla, et al., Phys. Rev. Lett. 110 (2013) 172501.

[48] C. Domingo-Pardo, et al., Nucl. Instrum. Methods Phys. Res. A 694 (2012) 297.

[49] Zs. Podolyák, et al., Nucl. Phys. A 722 (2003) 273c.

[50] E. Farnea, et al., Nucl. Instrum. Methods Phys. Res. A 621 (2010) 331.

[51] D. Ralet, et al., Phys. Rev. C 95 (2017) 034320.

[52] T. Alexander, et al., Acta Phys. Polon. B 46 (2015) 601.

[53] Zs. Podolyák, Int. J. of Mod. Phys. E 15 (2006) 1967.

[54] A. Boso, et al., Phys. Lett. B 797 (2019) 134835.

[55] R. Avigo, et al., Phys. Lett. B 811 (2020) 135951.

[56] P. Napiralla, et al., Eur. Phys. J. A 56 (2020) 147.

[57] Zs. Podolyák, et al., Phys. Rev. Lett. 117 (2016) 222302. 
[58] E. Clément, et al., Nucl. Instrum. Methods Phys. Res. A 855 (2017) 1.

[59] J.-M. Régis, et al., Nucl. Instrum. Methods Phys. Res. A 622 (2010) 83.

[60] O.J. Roberts, et al., Nucl. Instrum. Methods Phys. Res. A 748 (2014) 91.

[61] M. Rudigier, et al., Nucl. Instrum. Methods Phys. Res. A 969 (2020) 163967.

[62] A. Maj, et al., Acta Phys. Polon. B 40 (2009) 565.

[63] A. Dewald, O. Möller, P. Petkov, Progress in Part. and Nucl. Phys. 67 (2012) 786.

[64] J. Ljungvall, et al., Nucl. Instrum. Methods Phys. Res. A 679 (2012) 61.

[65] G. Jaworski, et al., Nucl. Instrum. Methods Phys. Res. A 673 (2012) 64.

[66] T. Hüyük, et al., Eur. Phys. J. A 52 (2016) 55.

[67] J.J. Valiente-Dobon, et al., Nucl. Instrum. Methods Phys. Res. A 927 (2019) 81.

[68] O. Skeppstedt, et al., Nucl. Instrum. Methods Phys. Res. A 421 (1999) 531.

[69] J. Scheurer, et al., Nucl. Instrum. Methods Phys. Res. A 385 (1997) 501.

[70] The GASPARD project. URL http://gaspard.in2p3.fr/index.html.

[71] D. Bazzacco, Nucl. Phys. A 746 (2004) 248.

[72] M. Ciemala, et al., Phys. Rev. C 101 (2020) 021303

[73] S.R. Stroberg, et al., Phys. Rev. Lett. 126 (2020) 022501.

[74] G. Hagen, et al., Phys. Rev. Lett. 108 (2012) 242501.

[75] K. Hebeler, J.D. Holt, J. Menendez, A. Schwenk, Annu. Rev. Nucl. Part. Sci. 65 (2015) 457.

[76] T. Otsuka, et al., Phys. Rev. Lett. 105 (2010) 032501.

[77] H.T. Fortune, Phys. Rev. C 88 (2013) 034310.

[78] G. Hagen, T. Papenbrock, M. Hjorth-Jensen, D.J. Dean, Rep. Progr. Phys. 77 (2014) 09630.

[79] M. Ciemala, et al., Eur. Phys. J. A 57 (2021) 156.

[80] S. Pullanhiotan, et al., Nucl. Instrum. Methods Phys. Res. A 593 (2008) 343.

[81] D.R. Entem, R. Machleidt, Phys. Rev. C 68 (2003) 041001(R); J. Simonis, K. Hebeler, J.D. Holt, J. Menendez, A. Schwenk, Phys. Rev. C 93 (2016) 011302(R).

[82] R.F. Garcia Ruiz, et al., Phys. Rev. C 91 (2015) 041304(R).

[83] A. Klose, et al., Phys. Rev. C 99 (2019) 061301(R).

[84] B. Pritychenko, M. Birch, B. Singh, M. Horoi, At. Data Nucl. Data Tables 107 (2016) 1.

[85] S.R. Stroberg, S.K. Bogner, H. Hergert, J.D. Holt, Annu. Rev. Nucl. Part. Sci. 69 (2019) 307.

[86] S.R. Stroberg, H. Hergert, J.D. Holt, S.K. Bogner, A. Schwenk, Phys. Rev. C 93 (2016) 051301(R).

[87] S.R. Stroberg, et al., Phys. Rev. Lett. 118 (2017) 032502.

[88] J. Simonis, S.R. Stroberg, K. Hebeler, J.D. Holt, A. Schwenk, Phys. Rev. C 96 (2017) 014303.

[89] D. Tilley, H. Weller, C. Cheves, R. Chasteler, Nucl. Phys. A 595 (1) (1995) 1-170.

[90] B. Pritychenko, M. Birch, B. Singh, M. Horoi, At. Data Nucl. Data Tables 107 (2016) 1-139.

[91] S. Heil, et al., Phys. Lett. B 809 (2020) 135678.

[92] A. Navin, et al., Phys. Rev. Lett. 85 (2000) 266.

[93] C. Thibault, et al., Phys. Rev. C 12 (1975) 644.

[94] D. Guillemaud-Mueller, et al., Nucl. Phys. A 426 (1984) 37.

[95] B. Bastin, et al., Phys. Rev. Lett. 99 (2007) 022503.

[96] K. Tshoo, et al., Phys. Rev. Lett. 109 (2012) 022501.

[97] F. Wienholtz, et al., Nature 498 (2013) 346; 500 (2013) 612, (erratum).

[98] D. Steppenbeck, et al., Nature 502 (2013) 207.

[99] A. Huck, et al., Phys. Rev. C 31 (1985) 2260.

[100] F. Nowacki, A. Obertelli, A. Poves, Prog. Part. Nucl. Phys. (2021) (accepted for publication),

[101] T. Otsuka, et al., Phys. Rev. Lett. 87 (2001) 082502.

[102] K.T. Flanagan, et al., Phys. Rev. Lett. 103 (2009) 142501.

[103] S. Franchoo, et al., Phys. Rev. C 64 (2001) 054308.

[104] U. Köster, et al., Phys. Rev. C 84 (2011) 034320.

[105] K.-H. Speidel, et al., Phys. Lett. B 633 (2006) 219

[106] A. Goldkuhle, et al., Phys. Rev. C 100 (2019) 054317.

[107] L.A. Riley, et al., Phys. Rev. C 96 (2017) 064315.

[108] O. Perru, et al., Phys. Rev. Lett. 96 (2006) 232501.

[109] S.M. Lenzi, F. Nowacki, A. Poves, K. Sieja, Phys. Rev. C 82 (2010) 054301.

[110] J. Ljungvall, et al., Phys. Rev. C 81 (2010) 061301(R).

[111] W. Rother, et al., Phys. Rev. Lett. 106 (2011) 022502.

[112] N. Aoi, et al., Phys. Rev. Lett. 102 (2009) 012502.

[113] T. Baugher, et al., Phys. Rev. C 86 (2012) 011305(R).

[114] J. Van de Walle, et al., Phys. Rev. C 79 (2009) 014309.

[115] M. Niikura, et al., Phys. Rev. C 85 (2012) 054321.

[116] C. Louchard, et al., Phys. Rev. C 87 (2013) 054302.

[117] J. Dechargé, D. Gogny, Phys. Rev. C 21 (1980) 1568.

[118] J.F. Berger, M. Girod, D. Gogny, Comput. Phys. Comm. 63 (1991) 365.

[119] M. Honma, T. Otsuka, T. Mizusaki, M. Hjorth-Jensen, Phys. Rev. C 80 (2009) 064323.

[120] K.L. Jones, et al., Nature 465 (2010) 454.

[121] J. Hakala, et al., Phys. Rev. Lett. 101 (2008) 052502.

[122] M. Wang, et al., Chin. Phys. C 36 (2012) 1603.

[123] M.-G. Porquet, et al., Eur. Phys. J. A 39 (2009) 295.

[124] Y.H. Zhang, et al., Phys. Rev. C 70 (2004) 024301.

[125] G. de Angelis, et al., Nucl. Phys. A 787 (2007) 74c.

[126] E. Sahin, et al., Nucl. Phys. A 893 (2012) 1.

[127] J. Van de Walle, et al., Phys. Rev. Lett. 99 (2007) 142501.

[128] K. Sieja, F. Nowacki, Phys. Rev. C 85 (2012) 051301R.

[129] R. Taniuchi, et al., Nature 569 (2019) 53.

[130] D. Verney, et al., Phys. Rev. C 76 (2007) 054312.

[131] M. Czerwinski, et al., Phys. Rev. C 88 (2013) 044314. 
[132] T. Materna, et al., Phys. Rev. C 92 (2015) 034305.

[133] J. Litzinger, et al., Phys. Rev. C 92 (2015) 064322.

[134] J. Litzinger, et al., Phys. Rev. C 97 (2018) 044323.

[135] K. Sieja, et al., Phys. Rev. C 88 (2013) 034327.

[136] M. Lettman, et al., Phys. Rev. C 96 (2017) 011301(R.

[137] O. Sorlin, M.-G. Porquet, Prog. Part. Nucl. Phys. 61 (2008) 602.

[138] D.K. Sharp, et al., Phys. Rev. C 87 (2013) 014312.

[139] K. Haravu, et al., Phys. Rev. C 1 (1970) 938.

[140] J.S. Thomas, et al., Phys. Rev. C 71 (2007) 044302.

[141] F. Didierjean, et al., Phys. Rev. C 96 (2017) 044320.

[142] F. Nowacki, et al., Phys. Rev. Lett. 117 (2016) 272501.

[143] J. Dudouet, et al., Phys. Rev. C 100 (2019) 011301(R).

[144] N. Jonson, et al., Nucl. Phys. A 371 (1981) 333.

[145] M. Siciliano, et al., Phys. Lett. B 806 (2020) 135474.

[146] D. Entem, R. Machleidt, Phys. Lett. B 524 (2002) 93.

[147] S. Bogner, et al., Phys. Rep. 386 (2003) 1.

[148] M. Dufour, A.P. Zuker, Phys. Rev. C 54 (1996) 1641.

[149] J. Duflo, A.P. Zuker, Phys. Rev. C 59 (1999) R2347.

[150] Y. Tsunoda, T. Otsuka, N. Shimizu, M. Honma, Y. Utsuno, Phys. Rev. C 89 (2014) 031301(R).

[151] C.J. Chiara, et al., Phys. Rev. C 91 (2015) 044309.

[152] B.P. Crider, et al., Phys. Lett. B 763 (2016) 108.

[153] C.J. Prokop, et al., Phys. Rev. C 92 (2015) 061302(R).

[154] S. Suchyta, et al., Phys. Rev. C 89 (2014) 021301(R).

[155] S. Leoni, et al., Phys. Rev. Lett. 118 (2017) 162502.

[156] N. Marginean, et al., Phys. Rev. Lett. 125 (2020) 102502.

[157] F. Recchia, et al., Phys. Rev. C 94 (2016) 054324.

[158] O. Wieland, et al., Phys. Rev. C 98 (2018) 064313.

[159] B. Elman, et al., Phys. Rev. C 100 (2019) 034317.

[160] I. Stefanescu, et al., Phys. Rev. Lett. 100 (2008) 112502.

[161] A.M. Oros-Peusquens, P.F. Mantica, Nucl. Phys. A 669 (2000) 81.

[162] E. Sahin, et al., Phys. Rev. C 91 (2015) 034302.

[163] V. Modamio, et al., Phys. Rev. C 88 (2013) 044326.

[164] M. Klintefjord, et al., Phys. Rev. C 95 (2017) 024312

[165] G. Bocchi, et al., Phys. Rev. C 89 (2014) 054302.

[166] S. Leoni, A. Bracco, G. Colo, B. Fornal, Eur. Phys. J. A (2019) 55.

[167] D. Bucurescu, et al., Nucl. Instrum. Methods Phys. Res. A 837 (2016) 1.

[168] A. Bohr, B.R. Mottelson, Nuclear Structure, World Scientific, 1998.

[169] D. Ralet, et al., Phys. Lett. B 797 (2019) 134797.

[170] E. Grosse, et al., Nucl. Phys. A 174 (1971) 525.

[171] O. Häusser, et al., Nucl. Phys. A 194 (1972) 113.

[172] C. Shand, et al., Acta Phys. Polon. B 46 (2015) 619.

[173] M. Rejmund, et al., Eur. Phys. J. A 8 (2000) 161.

[174] A. Bracco, E.G. Lanza, A. Tamii, Prog. Part. Nucl. Phys. 106 (2019) 360.

[175] X. Roca-Maza, N. Paar, Prog. Part. Nucl. Phys. 101 (2018) 96.

[176] D. Savran, T. Aumann, A. Zilges, Prog. Part. Nucl. Phys. 70 (2013) 210.

[177] F.C.L. Crespi, et al., Phys. Rev. Lett. 113 (2014) 012501.

[178] L. Pellegri, et al., Phys. Lett. B 738 (2014) 519.

[179] F.C.L. Crespi, et al., Phys. Rev. C 91 (2015) 024323.

[180] A. Bracco, F.C.L. Crespi, E.G. Lanza, Eur. Phys. J. A 51 (2015) 99.

[181] M. Krzysiek, et al., Phys. Rev. C 93 (2016) 044330.

[182] D. Savran, et al., Phys. Rev. Lett. 97 (2006) 172502.

[183] J. Endres, et al., Phys. Rev. Lett. 105 (2010) 212503.

[184] J. Endres, et al., Phys. Rev. C 80 (2009) 034302.

[185] K. Govaert, et al., Phys. Rev. C 57 (1998) 2229.

[186] S. Volz, et al., Nucl. Phys. A 779 (2006) 1.

[187] C. Loelius, et al., Phys. Rev. Lett. 121 (2018) 262501.

[188] V.Y. Ponomarev, et al., Nucl. Phys. A 323 (1979) 446

[189] M.D. Jones, et al., Phys. Rev. C 97 (2018) 024327.

[190] A.C. Larsen, et al., Phys. Rev. Lett. 111 (2013) 242504.

[191] L. Pellegri, et al., Phys. Rev. C 92 (2015) 014330.

[192] M. Spieker, et al., Phys. Lett. B 752 (2016) 102.

[193] N. Tsoneva, H. Lenske, Phys. Lett. B 695 (2011) 174.

[194] A. Bracco, F. Camera, Phys. Scripta. 91 (2016) 083002.

[195] S. Ceruti, et al., Phys. Rev. C 95 (2017) 014312.

[196] S. Ceruti, et al., Phys. Rev. Lett. 115 (2015) 222502.

[197] D. Pandit, et al., Phys. Lett. B 713 (2012) 434.

[198] D. Pandit, et al., Phys. Rev. C 88 (2013) 054327.

[199] S.M. Polikhanov, et al., Sov. Phys.-JETP 15 (1962) 1016.

[200] V. Metag, D. Habs, H.J. Specht, Phys. Rep. 65 (1980) 1.

[201] E.F. Moore, et al., Phys. Rev. Lett. 63 (1989) 360.

[202] P.J. Twin, et al., Phys. Rev. Lett. 57 (1986) 811.

[203] D. Rudolph, et al., Phys. Rev. Lett. 82 (1999) 3763.

[204] E. Ideguchi, et al., Phys. Rev. Lett. 87 (2001) 222501.

[205] T.L. Khoo, et al., Phys. Rev. Lett. 76 (1996) 1583.

[206] S. Biswas, et al., Phys. Rev. C 99 (2019) 064302. 
[207] S. Biswas, et al., Phys. Rev. C 102 (2020) 014326

[208] H.G. Kruse, B.H. Wildenthal, Bull. Am. Phys. Soc. 27 (1982) 533.

[209] E. Teruya, et al., Phys. Rev. C 92 (2015) 034320.

[210] J. Menendez, A. Poves, E. Caurier, F. Nowacki, Nucl. Phys. A 818 (2009) 139.

[211] Ch. Theisen, P.T. Greenlees, T.-L. Khoo, P. Chowdhury, T. Ishii, Nucl. Phys. A 944 (2015) 333.

[212] A. Sobiczewski, I. Muntian, Z. Patyk, Phys. Rev. C 63 (2001) 034306.

[213] B. Nerlo-Pomorska, K. Pomorski, J. Bartel, Phys. Rev. C 84 (2011) 044310.

[214] T.M. Shneidman, G.G. Adamian, N.V. Antonenko, R.V. Jolos, Phys. Rev. C 74 (2006) 034316.

[215] J.-P. Delaroche, M. Girod, H. Goutte, J. Libert, Nucl. Phys. A 771 (2006) 103.

[216] A. Lopez-Martens, et al., Phys. Lett. B 380 (1996) 18.

[217] K. Hadynska-Klek, et al., Phys. Rev. Lett. 117 (2016) 062501.

[218] K. Hadynska-Klek, et al., Phys. Rev. C 97 (2018) 024326.

[219] K. Hadynska-Klek, et al., Acta Phys. Polon. B 44 (2013) 617.

[220] J. Srebny, D. Cline, Int. J. Modern Phys. E 20 (2011) 422.

[221] E. Ideguchi, et al., Phys. Rev. Lett. 87 (2001) 222501.

[222] C.E. Svensson, et al., Phys. Rev. Lett. 85 (2000) 2693.

[223] J. Styczen, et al., Nucl. Phys. A 262 (1976) 317.

[224] P. Bednarczyk, et al., Eur. Phys. J. A 2 (1998) 157.

[225] S.M. Lenzi, et al., Phys. Rev. C 60 (1999) 021303.

[226] C. O'Leary, et al., Phys. Lett. B 459 (1999) 73

[227] P.-A. Söderström, et al., Phys. Rev. C 86 (2012) 054320.

[228] E. Cheifetz, R.C. Jared, S.G. Thompson, J.B. Wilhelmy, Phys. Rev. Lett. 25 (1970) 38.

[229] P. Federman, S. Pittel, Phys. Lett. B 69 (1977) 385.

[230] P. Federman, S. Pittel, Phys. Rev. C 20 (1979) 820.

[231] U. Hager, et al., Nucl. Phys. A 793 (2007) 20.

[232] S. Naimi, et al., Phys. Rev. Lett. 105 (2010) 032502.

[233] N. Mărginean, et al., Phys. Rev. C 80 (2009) 021301.

[234] M. Albers, et al., Phys. Rev. Lett. 108 (2012) 062701.

[235] J. Dudouet, et al., Phys. Rev. Lett. 118 (2017) 162501.

[236] R.F. Casten, Nuclear Structure from a Simple Perspective, Oxford University Press, 2000, pp.194, 233.

[237] T.R. Rodriguez, Phys. Rev. C 90 (2014) 034306.

[238] J. Xiang, et al., Phys. Rev. C 93 (2016) 054324.

[239] J.-P. Delaroche, et al., Phys. Rev. C 81 (2010) 014303.

[240] P.R. John, et al., Phys. Rev. C 95 (2017) 064321.

[241] P.A. Soederstroem, et al., Phys. Rev. C 81 (2010) 034310.

[242] B. Fogelberg, et al., Phys. Rev. Lett. 73 (1994) 2413.

[243] S.E.A. Orrigo, Eur. Phys. J. Web Conf. 121 (2016) 06006.

[244] E. Aprile, et al., Phys. Rev. Lett. 122 (2019) 071301.

[245] J. Angle, et al., Phys. Rev. Lett. 101 (2008) 091301.

[246] J. Menendez, et al., Phys. Rev. D 86 (2012) 103511

[247] P. Toivanen, et al., Phys. Rev. C 79 (2009) 044302.

[248] M.T. Ressell, D.J. Dean, Phys. Rev. C 56 (1997) 535.

[249] E. Aprile, et al., Phys. Rev. Lett. 111 (2013) 021301.

[250] B.A. Brown, W.D.M. Rae, Nucl. Data Sheets 120 (2014) 115.

[251] Ashok Kumar Jain, et al., Nucl. Data Sheets 128 (2015) 1.

[252] S. Chakraborty, et al., Phys. Rev. C 97 (2018) 054311.

[253] Evaluated Nuclear Structure Data File, https://www.nndc.bnl.gov/ensdf.

[254] P. Walker, G. Dracoulis, Nature 399 (1999) 35.

[255] A. Vogt, et al., Phys. Rev. C 93 (2016) 054325.

[256] A. Vogt, et al., Phys. Rev. C 95 (2017) 024316.

[257] A. Vogt, et al., Phys. Rev. C 96 (2017) 024321.

[258] L. Kaya, et al., Phys. Rev. C 98 (2018) 014309.

[259] L. Kaya, et al., Phys. Rev. C 98 (2018) 054312.

[260] L. Kaya, et al., Phys. Rev. C 100 (2019) 024323.

[261] L. Kaya, et al., Phys. Rev. C 99 (2019) 014301.

[262] J. Genevey, et al., Phys. Rev. C 65 (2002) 034322.

[263] B. Fogelberg, et al., AIP Conf. Proc. 447 (1998) 191.

[264] M. Ferraton, et al., Eur. Phys. J. A 35 (2008) 167.

[265] J.A. Pinston, et al., Phys. Rev. C 61 (2000) 024312.

[266] A. Kerek, et al., Nucl. Phys. A 172 (1971) 603.

[267] A. Zemel, et al., Z. Phys. A 304 (1982) 269.

[268] J. Gizon, et al., Nucl. Phys. A 222 (1974) 557.

[269] J. Gizon, et al., Nucl. Phys. A 252 (1975) 509.

[270] N.J. Stone, At. Data Nucl. Data Tables 90 (2005) 75.

[271] S. Harissopulos, et al., Phys. Rev. C 52 (1995) 1796.

[272] J.J. Valiente-Dobon, et al., Phys. Rev. C 69 (2004) 024316.

[273] M.N. Tantawy, et al., Phys. Rev. C 73 (2006) 024316.

[274] A. Astieret, et al., Eur. Phys. J. A 22 (2014) 2.

[275] J. Genevey, et al., Phys. Rev. C 63 (2001) 054315.

[276] H.F. Brinckmann, et al., Nucl. Phys. A 96 (1967) 318.

[277] T. Matsuzawa, et al., Phys. Rev. C 62 (2006) 054304.

[278] R.H. Mayer, et al., Z. Phys. A 34 (1992) 247.

[279] H. Gausemel, et al., Phys. Rev. C 69 (2004) 054307.

[280] C.T. Zhang, et al., Z. Phys. A 353 (1995) 11.

[281] B.A. Brown, et al., Phys. Rev. C 71 (2005) 044317. 
[282] R. Wyss, et al., Nucl. Phys. A 505 (1989) 337.

[283] R.E. Peierls, et al., Nuclear Phys. 38 (1962) 154.

[284] R.F. Casten, Nuclear Structure from a Simple Perspective, Oxford science publications, 2000.

[285] H. Morinaga, Neil L. Lark, Nuclear Phys. 67 (1965) 315.

[286] A. Granderath, et al., Nucl. Phys. A 597 (1996) 427.

[287] R.F. Casten, P. Von Brentano, Phys. Lett. B 152 (1985) 22.

[288] R. Wyss, et al., Phys. Lett. B 215 (1988) 211.

[289] E.S. Paul, et al., Phys. Rev. C 40 (1989) 1255.

[290] Y. Huang, et al., Phys. Rev. C 93 (2016) 064315.

[291] J.G. Wang, et al., Phys. Rev. C 75 (2007) 064301.

[292] R.S. Chakrawarthy, Pramana 53 (1999) 53.

[293] NuDat 2.7, https://www.nndc.bnl.gov/nudat2.

[294] R.O. Sayer, et al., At. Data Nucl. Data Tables 15 (1975) 85.

[295] C.S. Purry, et al., Nucl. Phys. A 632 (1998) 229.

[296] A. Johnson, et al., Nucl. Phys. A 179 (1972) 753.

[297] A. Dewald, et al., Phys. Rev. C 37 (1988) 289.

[298] Nucl. Data Sheets 120 (2014) 115.

[299] N. Shimizu, arXiv:1310.5431.

[300] E. Caurier, et al., Rev. Modern Phys. 77 (2005) 427.

[301] E. Caurier, et al., Phys. Rev. C 82 (2010) 064304.

[302] E. Caurier, F. Nowacki, A. Poves, Phys. Lett. B 711 (2012) 62.

[303] L. Coraggio, et al., Prog. Part. Nucl. Phys. 62 (2009) 135.

[304] L. Coraggio, et al., Ann. Phys. 327 (2012) 2125.

[305] K. Higashiyama, N. Yoshinaga, Phys. Rev. C 83 (2011) 034321.

[306] N. Yoshida, et al., Phys. Lett. B 114 (1982) 86.

[307] K. Nomura, et al., Phys. Rev. C 96 (2017) 014304.

[308] L.M. Robledo, et al., Phys. Rev. C 78 (2008) 034314.

[309] C.B. Moon, et al., Phys. Rev. C 76 (2007) 067301.

[310] C. Qi, Z.X. Xu, Phys. Rev. C 86 (2012) 044323.

[311] C. Qi, Phys. Rev. C 94 (2016) 034310.

[312] P.R. John, et al., Phys. Rev. C 90 (2014) 021301.

[313] C. Wheldon, et al., Phys. Rev. C 63 (2001) 011304(R).

[314] Zs. Podolyák, et al., Phys. Rev. C 79 (2009) 031305.

[315] B. Birkenbach, et al., Phys. Rev. C 92 (2015) 044319.

[316] A.V. Afanasjev, O. Abdurazakov, Phys. Rev. C 88 (2013) 014320.

[317] A.V. Afanasjev, Phys. Scr. 89 (2014) 054001.

[318] I. Wiedenhöver, et al., Phys. Rev. Lett. 83 (1999) 2143.

[319] W. Heisenberg, Z. für Phys. 77 (1932) 1.

[320] E. Wigner, Phys. Rev. 51 (1936) 106.

[321] M.A. Bentley, S.M. Lenzi, Progr. in Part. and Nucl. Phys. 59 (2007) 497.

[322] R.D.O. Llewellyn, et al., Phys. Lett. B 811 (2020) 135873.

[323] A. Poves, J. Sanchez-Solano, E. Caurier, F. Nowacki, Nucl. Phys. A 694 (2001) 157.

[324] E. Caurier, K. Langanke, G. Martinez-Pinedo, F. Nowacki, P. Vogel, Phys. Lett. B 522 (2001) 240.

[325] M.N. Harakeh, et al., Phys. Lett. B 176 (1986) 297.

[326] M. Kicinska-Habior, et al., Nucl. Phys. A 731 (2004) 138.

[327] A. Corsi, et al., Phys. Rev. C. 84 (2011) 041304(R).

[328] H. Sagawa, P.F. Bortignon, G. Colo, Phys. Lett. B 444 (1998) 1.

[329] W. Satuła, J. Dobaczewski, W. Nazarewicz, M. Rafalski, Phys. Rev. Lett. 106 (2011) 132502.

[330] N. Auerbach, Phys. Rev. C 79 (2009) 035502.

[331] J. Damgaard, Nucl. Phys. A 130 (1969) 233.

[332] I.S. Towner, J.C. Hardy, M. Harvey, Nucl. Phys. A 284 (1977) 269.

[333] I.S. Towner, J.C. Hardy, Phys. Rev. C 82 (2010) 065501.

[334] A. Kellerbauer, et al., Phys. Rev. Lett. 93 (2004) 072502.

[335] S. Frauendorf, A. Macchiavelli, Prog. Part. Nucl. Phys. 78 (2014) 24.

[336] B. Cederwall, et al., Phys. Rev. Lett. 124 (2020) 062501.

[337] N. Marginean, et al., Phys. Rev. C 63 (2001) 031303(R).

[338] H. Liang, J. Meng, S.-G. Zhou, Phys. Rep. 570 (2015) 1.

[339] C. Delafosse, et al., Phys. Rev. Lett. 121 (2018) 192502.

[340] C. Delafosse, et al., Acta Phys. Polon. B 50 (2019) 633.

[341] K. Sieja, T.R. Rodriguez, K. Kolos, D. Verney, Phys. Rev. C 88 (2013) 034327.

[342] T. Niksic, D. Vretenar, P. Ring, Phys. Rev. C 78 (2008) 034318.

[343] N. Pietralla, P. von Brentano, A. Lisetskiy, Prog. Part. Nucl. Phys. 60 (2008) 225.

[344] C.E. Alonso, J.M. Arias, L. Fortunato, N. Pietralla, A. Vitturi, Phys. Rev. C 78 (2008) 017301.

[345] C. Stahl, et al., Phys. Rev. C 92 (2015) 044324.

[346] P.M. Walker, Zs. Podolyák, Phys. Scripta. 95 (2020) 044004.

[347] B. Maheshwari, A.K. Jain, Phys. Lett. B 753 (2016) 122.

[348] M. Rejmund, et al., Phys. Lett. B 753 (2016) 86.

[349] V. Vandone, et al., Phys. Rev. C 88 (2013) 034312.

[350] T.P.D. Swan, et al., Phys. Rev. C 86 (2012) 044307.

[351] S.K. Tandel, et al., Phys. Rev. C 73 (2006) 044306.

[352] P.M. Walker, et al., Phys. Lett. B 408 (1997) 42.

[353] T. Døssing, et al., Phys. Rep. 268 (1996) 1.

[354] S. Leoni, et al., Phys. Rev. Lett. 93 (2004) 022501.

[355] S. Leoni, et al., Phys. Rev. Lett. 101 (2008) 142502.

[356] S. Leoni, et al., Phys. Rev. C 79 (2009) 064306. 
[357] S. Leoni, et al., Phys. Rev. C 79 (2009) 064307.

[358] M. Matsuo, et al., Nucl. Phys. A 736 (2004) 223.

[359] A. Bracco, et al., Phys. Rev. Lett. 76 (1996) 4484.

[360] A. Bracco, et al., Rep. Progr. Phys. 65 (2002) 299.

[361] S.K. Tandel, et al., Phys. Rev. C 77 (2008) 024313.

[362] L. Corradi, G. Pollarolo, S. Szilner, J. Phys. G: Nucl. Part. Phys. 36 (2009) 113101.

[363] D. Montanari, et al., Phys. Rev. C 93 (2016) 054623.

[364] D. Montanari, et al., Phys. Rev. Lett. 113 (2014) 052501.

[365] J.H. Sørensen, A. Winther, Nucl. Phys. A 550 (1992) 306.

[366] F. Videbaek, Ole Hansen, B.S. Nilsson, E.R. Flynn, J.P. Peng, Nucl. Phys. A 433 (1985) 441.

[367] N. Lalovic, et al., J. Phys. G 45 (2018) 035105.

[368] E.C. Simpson, J.A. Tostevin, Zs. Podolyák, P.H. Regan, S.J. Steer, Phys. Rev. C 80 (2009) 064608.

[369] E.C. Simpson, J.A. Tostevin, Zs. Podolyák, P.H. Regan, S.J. Steer, Phys. Rev. C 82 (2010) 037602.

[370] D. Bachelier, et al., Phys. Lett. B 172 (1986) 23.

[371] D. Yan, J. Huo, Nucl. Data Sheets 121 (2014) 1.

[372] Zs. Podolyák, et al., Proceedings of Science, IMPC2016, 2017, p. 231.

[373] NUPECC Long Range Plan 2017; http://www.nupecc.org.

[374] F. Gramegna, Il Nuovo Cimento 42C (2019) 61; https://web.infn.it/spes/.

[375] https://fair-center.eu/.

[376] H. Geissel, et al., Nucl. Instrum. Methods Phys. Res. B 204 (2003) 71.

[377] Zs. Podolyák, Nucl. Instrum. Methods Phys. Res. B 266 (2008) 4589.

[378] https://www.ganil-spiral2.eu/en/.

[379] M.J.G. Borge, K. Riisager, Eur. Phys. J. A 52 (2016) 334, http://hie-isolde-project.web.cern.ch. 